\title{
The Social Construction of Ageing Masculinities in Neoliberal Society - Reflections on Retired German Men
}

\author{
Lisa-Nike Bühring
}

A thesis submitted to The University of Gloucestershire in accordance with the requirements of the degree of Doctor of Philosophy in the School of Media

August 2020

Word Count: 80,321 


\section{Table of Contents}

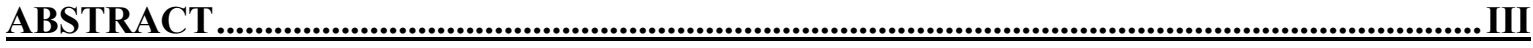

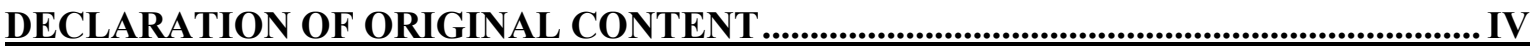

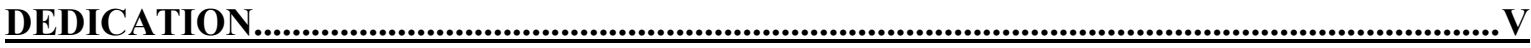

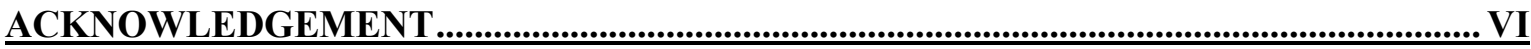

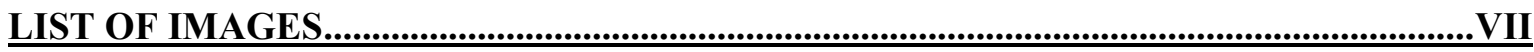

1 INTRODUCTION

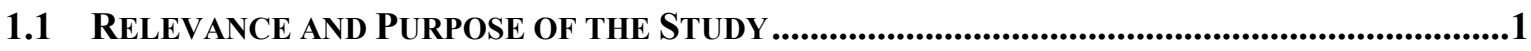

1.2 ASSUMPTIONS, LIMITATIONS AND STRUCTURE OF THE STUDY ...............................................7

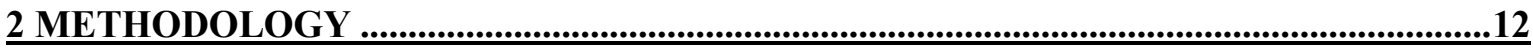

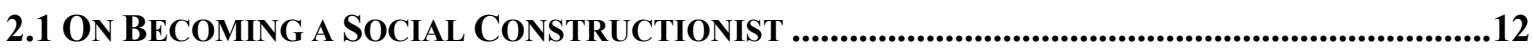

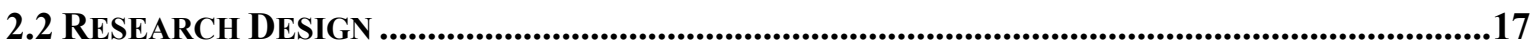

3 THE CONSTRUCTION OF AGEING IN NEOLIBERAL SOCIETIES ..............................25

3.1 NEOLIBERAL MASTER NARRATIVES \& INDIVIDUAL IDENTITY CONSTRUCTIONS..................25

3.2 NEOLIBERAL MASTER NARRATIVES OF OLDER AGE ..................................................................31

\section{THE CONSTRUCTION OF MEN AND MASCULINITIES IN NEOLIBERAL}

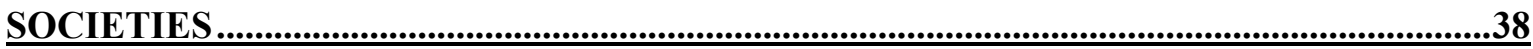

4.1 The Construction Of Hegemonic Masculinities in the West OR Why do Men

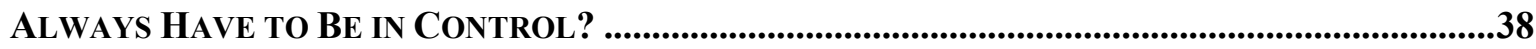

4.2 HEGEMONIC CONSTRUCTIONS OF GERMAN MASCULINITIES...................................................42

4.3 Controlling the UnControllable: OLder Age \& Hegemonic Constructions of

MASCULINITY IN NEOLIBERAL SOCIETIES.......................................................................................47

\section{THE CONSTRUCTION OF MALE AGEING IN THE GERIACTION FILM TRILOGY}

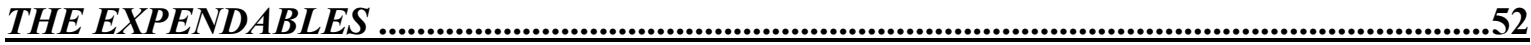

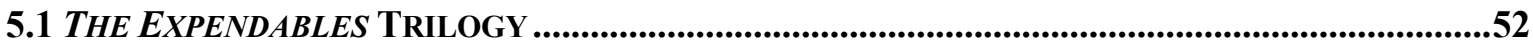

5.2 NARRATIVES OF MASCULINITY IN THE EXPENDABLES TRILOGY .........................................55

5.3 NARRATIVES OF MALE AgEING IN THE EXPENDABLES TRILOGY .....................................62

5.4 NeOLIBERALISM \& THE CONSTRUCTION OF MALE AgEING IN THE EXPENDABLES TRILOGY 
6.1 General REALISATIONS, RESEARCHER's POSITION \& INITIAL DEVELOPMENT OF INTERVIEW QUESTIONS . .76

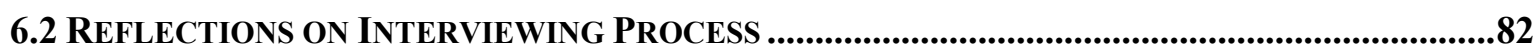

6.3 REFLECTIONS ON INTERVIEW ANALYSIS......................................................................92

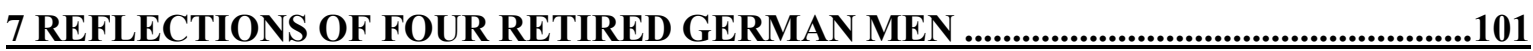

7.1 THE INTERVIEWEES .101

7.2 REFLECTIONS ON MASCULINITY, FEMININITY \& AGEING ...............................................106

7.3 REFLECTIONS ON AgEING, MASCULINITY \& CONTROL

7.4 REFlections on SOCIO-CULTURAL Understandings OF OLder Age \& MASCULINITY

8.1 OLder Men Staring AT Age In A NEOLIBERAL SOCIETY .............................................153

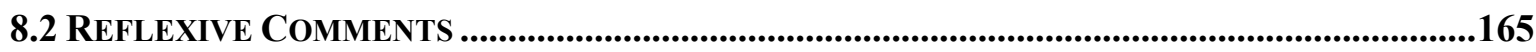

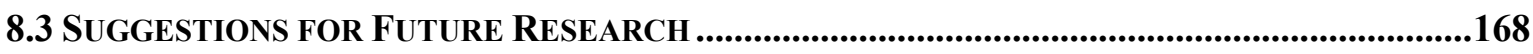

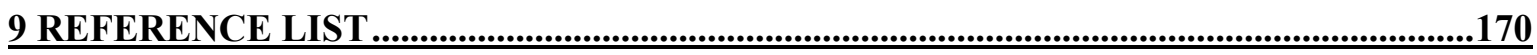

APPENDIX

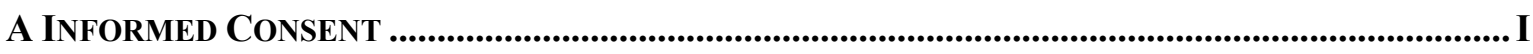

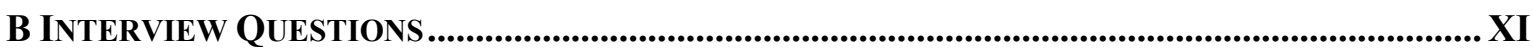

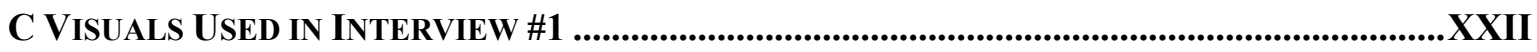

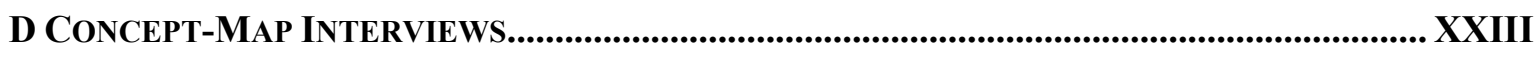

E REFLECTIONS ON INTERVIEWING PROCESS ......................................................................... XXIV

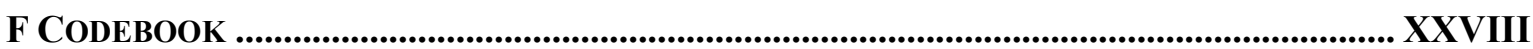

G INTERVIEW \& TRANSLATED EXTRACTS BURKHARD ROSENFELD .................................... XXIX

H INTERVIEW \& TRANSLATEd EXTRACTS PROF. DR GÜNTER SCHOTTEN ............................... LIII

I INTERVIEW \& TRANSLATED EXTRACTS GB ..............................................................LXXIX

J INTERVIEW \& TRANSLATED EXTRACTS DR LARS BÜHRING ........................................ CVIII

K CONCEPT-MAP THEMATIC UNITS MASCULINITY \& FEMININITY ................................... CXXXII

L CONCEPT-MAP THEMATIC UNITS CONTROL \& AUTONOMY ........................................... CXXXII

M CONCEPT-MAP THEMATIC UNITS AGEING AS DECLINE ........................................ CXXXIII

N CONCEPT-MAP THEMATIC UNITS SUCCESSFUL AGEING ..........................................CXXXIV

O CONCEPT-MAP THEMATIC UNITS SOCIETY, CULTURE \& MEDIA................................ CXXXV

P CONCEPT-MAP THEMATIC UNITS GENERATIONAL CONFLICT.....................................CXXXVI 


\section{Abstract}

Low fertility rates, advances in medicine and improved living standards have dramatically altered the worldwide demographic landscape of age and ageing. The world's population is growing older, and as a result of this demographic trend, scholarly research in a variety of academic disciplines in the West have turned their focus towards socio-cultural understandings of old age.

Research-based in cultural gerontology suggests that within the context of western neoliberal societies perceptions of older age mainly unfold within two hegemonic narratives, namely ageing as related to decline, frailty and dependency and successful ageing characterised by youthfulness, productivity and continued personal autonomy. Since recent multi-disciplinary approaches to age presume that western views of ageing are shaped by the socio-cultural environment and its hegemonic narratives, the role media play in the dissemination and preservation of these hegemonic narratives has been an important site of investigation, particularly in relation to the portrayal of older women. However, the media depiction of older males and related cultural narratives and how older men experience these narratives within a cultural environment other than the U.S. have received less academic attention. Paradoxically, whilst the representation of older male ageing is strikingly less in the focus of scholarly debate than female ageing, the action film genre has recently brought older male characters into focus through the revival of tough-guy action films featuring older male protagonists.

This thesis analyses three 'geri-action films', The Expendables, The Expendables 2 and The Expendables 3, in order to explore current representational contextualisations of masculine ageing within the hegemonic socio-cultural constructions of successful ageing and ageing as decline. In so doing, it furthers an understanding of the dominant socio-cultural frames of reference which influence older men's constructions of older male identities. Subsequently, this thesis explores the ways that ageing impacts on the later lives of men who would have been judged during their working lives to fulfil the criteria of hegemonic masculinity in that they are heterosexual, white and were, before retirement, in white-collar, affluent, middleand upper-managerial positions. The in-depth semi-structured interviews with four retired German men offered genuine and novel views of older German men's inner worlds in relation to the specificities of their life-course narratives and self-perceptions within the socio-cultural and theoretical contexts of ageing and masculinity.

Through qualitative research underpinned by theoretical and conceptual understandings of media, ageing and representation, cultural gerontology and masculinity studies, this thesis offers a critical analysis of previously unheard narratives about ageing and masculinity. 


\section{Declaration of Original Content}

I declare that the work in this thesis was carried out in accordance with the regulations of the University of Gloucestershire and is original except where indicated by specific reference in the text. No part of the thesis has been submitted as part of any other academic award. The thesis has not been presented to any other education institution in the United Kingdom or overseas.

Any views expressed in the thesis are those of the author and in no way represent those of the University.

Signed Date 06.08.2020

doi: 10.46289/MEDA0012 
Dedication

To Eri and Lars- you are at the heart of everything I am and do 


\section{Acknowledgement}

First of all, I would like to thank my parents.

Papi: Thank you for your steadfast belief in me, for your endless patience and understanding and for all the intense conversations we had, which aided me enormously in developing this thesis. Also, thank you so much for never losing trust in me finishing this project and the support, stability and security you provided me with. I really hope that the outcome of the past five years was worth waiting for.

Nani: Sadly, you only saw the beginning of my exciting 5-year-journey, but it was you who instilled a belief in me that I can succeed in everything I choose to do provided I really put my mind to it without which I would have never dared to approach this project. Wherever you are, I hope I could make you a little proud.

I want to extend my gratitude to my wonderful, inspiring and exceedingly knowledgeable supervisors, Ros Jennings and Hannah Grist. Your support, understanding and encouragement helped me through all the challenges I encountered. I learned so much from you, and it is mainly due to you that the past years were an exciting and eye-opening experience for me which enabled me to develop myself professionally and personally. There is no way that I could have finalised this project without your help. I cannot thank you enough for being there for me.

Moreover, I am exceedingly grateful for the openness and honesty of my interview participants and for the trust they honoured me with. That you shared your life-course narratives with me without any reservations made this study exciting, relevant and unique. I would also like to thank Theresa Kuldova who was such a valuable and patient mentor to me and Laxmi Remer who made it crystal clear to me that the only way forward was to approach a doctoral thesis even if this meant giving up some independence.

Last but by no means least, I would like to thank my friends. Marcel: thank you so much for your valuable input, for putting up with my moods and for being there. Nadine, Laura and Daniel: thank you so much for listening to me and for all the encouragement I received from you. You are wonderful, and I count myself extremely lucky to have friends like you. 


\section{List of Images}

Illustration 1: Research Approach.. 


\section{Introduction}

\subsection{Relevance and Purpose of the Study}

Not long ago when I presented a paper about the construction of female ageing in the film R.E.D. (Schwenkte, 2010) at a conference in the UK, one participant of African origin but living in the UK after my presentation said: “But isn't it totally normal, an evolutionary thing, that one prefers the green grass and not the dry grass. ... I mean in my country older age is honoured highly but still, something fresh is always better than something old, that is a natural feeling." (Anonymous, Personal Comment, Conference Contention, Brighton University, 14.09.2018). This remark stands representative for many similar comments I heard in different contexts, but usually by people my age or younger when I had mentioned the topic of my doctoral thesis.

But is it really 'natural ' to view older age as something abject and negative, which is linked to an inability, a decrease of physical and mental fitness and general decline? It might appear to be natural when you still feel and are considered to be young, but based on conversations I had with older people, not just within the context of the interviews I conducted, it is not once one has reached older age, which as defined by the World Health Organisation (WHO) is in western industrialised countries when approaching retirement at ages from 60 to 65 (2011a). So how is it possible to entertain a rather negative perception of ageing over long periods of one's life and then revise this view entirely as soon as one reaches older age? And more generally where do these understandings of ageing come from? And are there differences in the way men and women view themselves and are viewed by society in western countries in older age? As a result of the demographic changes presently taking place in developed countries, these and related question have not only motivated my own research but they are also increasingly in the centre of scholarly debates in a variety of academic fields (Arber, Davidson, \& Ginn, 2003; Beard et al., 2012; Blaikie, 1999; Fairclough-Isaacs, 2015; Featherstone, 1995; Kotter-Gruhn \& Hess, 2012; Lyons, 2009; Tulle, 2015; Victor, 1994).

The increase in public and academic attention for issues linked to older age is not overly surprising given the fact the markedly extended life span in industrialised countries will result in a $188 \%$ worldwide increase of people aged $65+$ and a $351 \%$ rise of people 85 or older between 2010 and 2050 (WHO, 2011b). In Germany over 20\% of the population was 65 or older in 2011 and this share is predicted to reach 30\% in 2060 (Hoffmann, Gordo, Nowossadeck, Simonson, \& Tesch-Römer, 2015). The percentage of old-age poverty in Germany is relatively low and percentages of consumption power as well as life satisfaction 
comparably high when compared to other EU countries (Haustein \& Mischke, 2011; Hoffmann et al., 2015; OECD, 2015).

Yet, public discourse in Germany paints a rather bleak image of older age and retirement characterised by a loss of economic as well cultural capital, whilst at the same time tirelessly warning the public that the increase in older people will drain the social security system so that the pension for future generations cannot be guaranteed. When considering the high visibility of issues linked to older age in public discussions (Finanzen100, 16.07.2018; Lewicki \& Wigger, 2013; Lietzmann, 2017), the moderate extent to which individual and socio-cultural perceptions of ageing are in the focus of academic work in Germany is striking (Kessler, 2009). This is particularly true when the research conducted in Germany is compared with the multiplicity of studies in other industrialised countries (Arber et al., 2003; Bengtson, Coleman, \& Kirkwood, 2005; Carroll \& Bartlett, 2015; Cruikshank, 2013; Fairclough-Isaacs, 2015; Gullette Morganroth, 2004; Segal, 2013; van Dyk, 2014) and even more so for research rooted in constructionist research philosophy and based on a qualitative approach. In line with both the predominant essentialist research orientation and the nature of public discourse in Germany most analyses with a specific focus on older age are statistical evaluations of demographic trends (Haustein \& Mischke, 2011; Hoffmann et al., 2015).

In contrast, recent approaches outside of Germany in the multi-disciplinary academic debate, particularly in cultural gerontology increasingly focuses on gaining a better understanding of older age as shaped by the socio-cultural environment and its hegemonic narratives and on exploring how these narrative impact individual identity constructions in older age (Arber et al., 2003; Baars, 2013; Cruikshank, 2013; Gullette Morganroth, 2004; Jennings \& Krainitzki, 2015; Segal, 2013; Tulle, 2015). Scholarly work in cultural gerontology questions the division of the life course by chronological stages such as young, middle-aged and old (Cruikshank, 2013) or by age cohorts such as X-ers or Baby Boomers (Gullette Morganroth, 2004) since it neither captures individal self-perception sufficiently nor the multitude of idiosyncratic concepts of later life (Baars, 2013; Moglen, 2008a). Our many different selves are often contradictory and seldom synchronous with our chronological age (Moglen, 2008a). They are expressed in the personal life-course narratives we construct for ourselves and share with others (McAdams, 2001) and are strongly influenced by the hegemonic narratives of the culture we live in (McAdams, 2001). The cultural narratives linked to ageing are based on chronological age and relate progress to young age, while middle age and old age typically associated with decline (Gullette Morganroth, 2004).

Although notions of decline have dominated socio-cultural understandings of ageing in the west for a long time (Segal, 2014; Wilson, 2000), the rising numbers of older people and the 
discovery of their potential on the consumer market, combined with the accelerating significance of neoliberal values has facilitated the emergence of another powerful frame for the construction of ageing. The successful ageing paradigm is a much more positively connoted concept than the prior understanding of ageing as merely being a time of natural demise preceding death. The concept of successful ageing is primarily based on transferring neoliberal conceptions of individual success indicative for earlier stages of one's life to older age (Featherstone, 1995; Gergen \& Gergen, 2000). As such, crucial criteria determining the success of individuals in the ageing process are retaining personal autonomy for as long as possible and by consuming the products and services necessary to enable physical and mental fitness into old age. This, in turn, allows older people to stay active and productive members of society (Gullette Morganroth, 2004; Katz \& Marshall, 2003; Lamb, RobbinsRuszkowski, Corwin, Calasanti, \& King, 2017). Whilst the development of a narrative offering an alternative to conventional views in which ageing is linked to decline must be seen as positive, the total disregard for the impact aspects of intersectionality, such as socioeconomic background, gender, race or disability, have on personal autonomy and thus, on fulfilling the standards set for successful ageing furthers discrimination and marginalisation. The illusion that ageing can be managed and controlled provided one tries hard enough has markedly amplified the pressure on older people and led to an even harsher exclusion of anyone who does not fulfil the standards of successful ageing (Cruikshank, 2013; Katz \& Calasanti, 2014; Lamb et al., 2017; Tulle, 2015; Wilson, 2000).

The narrative of successful ageing must, therefore, be evaluated as yet another neoliberal means of sorting people into categories which define their value on the basis of market criteria. As such, it is essentially based on enabling exclusion rather than inclusion (Harvey, 2007a). Understanding both the narratives of ageing as linked to decline and the narrative of successful ageing in more depth and exploring how these influence our self-perception is the prerequisite for viewing life not as a linear process based on chronological time, which defines what one should do and be and how one should behave and feel at any particular age. Can more differentiated views of older age informed by the personal accounts of older people and rooted in their conception of time lead to" [...] feel at home in the life course at every age?"(Gullette Morganroth, 2004, p. 20)

I do not know, but I am sure that understanding a phenomenon is a crucial first step for enabling social change. Initiating changes in the social-cultural view of older age is, in my view, especially relevant, not only because it affects everybody, provided one lives long enough, but also since current binary and stereotypical conceptions of ageing foster ageism and disable both fruitful intergenerational cooperation and an equal inclusion of every older person which would benefit the individual as well society as a whole (de Medeiros, 2016; 
Gill, Hamad, Kauser, Negra, \& Roshini, 2016; Gullette Morganroth, 2008; Gullette Morganroth, 2011; Hogan, 2016; Moglen, 2008a; Twigg \& Martin, 2015).

Feminist scholars and activists were not only among the first to critically examine the sociocultural perception of ageing and individual negotiations with the ageing process, but they were also pioneers in highlighting the inadequacy and inherent potential for discrimination and marginalisation of narratives linked to ageing. Susan Sontag's article The Double Standards of Ageing (1972) and Simone Beauvoir's book The Coming of Age (1972) were both published in the 70s and must be considered as seminal and trendsetting with regards to research conducted in ageing studies. Their focus on the gendered nature of ageing marked the beginning of a high number of analyses exploring the particular challenges and experiences involved in female ageing and the specific socio-cultural standards applied to female ageing (Arber et al., 2003; Calasanti \& Slevin, 2001; Clarke \& Bennett, 2015; Jermyn \& Holmes, 2015).

The disadvantaged position of women in the still patriarchal societies of the West continues to characterise older age. Women are evaluated on their appearance and on how well they have managed to maintain their looks since this is transmitted as the most fundamental basis for female identity and simultaneously determines the social capital of women (Dolan, 2018; Richardson, 2019). The perceived decrease in female attractiveness in older age, therefore, leads to social marginalisation and a social status increasingly characterised by invisibility and insignificance which seriously jeopardises a positive self-perception of many older women in the west (Clarke, 2010; Jermyn \& Holmes, 2015; Segal, 2014). In addition, as a result of a professional career development frequently characterised by part-time work, lower remuneration and interruptions due to maternity leave women are financially also often much worse off than their male counterparts in older age - something which further decreases their social value in societies defining the socio-cultural and politico-economic of individuals by their market value (Cruikshank, 2013; Wilson, 2000). There can, thus, be no doubt that the disadvantages and challenges indicative for the earlier stages of their female lives are written forth and in many ways amplified in older age. As such, a critical discussion of the socio-cultural narratives linked to female ageing and an exploration of how these affect older women is of utmost importance and relevance.

Notwithstanding this and despite the fact that men are in western societies indeed privileged and carry some of these privileges forward into older age, socio-cultural narratives of ageing also affect men, even if perhaps to a lesser degree than women (Ayalon \& Tesch-Römer, 2018; Calasanti \& King, 2005; Fung, 2013; Thompson, 2006). The view that gender roles are relational and that thus constructions of femininity and masculinity are inextricably linked has sparked a growing interest in the construction of masculinities in gender studies 
and particularly in the relatively new academic discipline of men's studies (Bordo, 1999; Burke, 2011; Flood, 1997; Greig, Kimmel, \& Lang, 2000; Hearn, 2004; Hirose, 2010; Thompson \& Whearty, 2004). Current approaches in this field have moved beyond the notion of understanding masculinity as a unified attribute, stable in space and time (Beynon, 2001; Connell, 2009; Coston \& Kimmel, 2012; Kimmel, 2001). Instead, maleness is viewed as a multiplicity of historical and life-course masculinities mutually shaped by individual actions and the cultural setting of a particular historical period (Connell, 2005). As such, masculinity is not understood as a genetic predisposition but progressively viewed as a multidimensional construct determined by the socio-cultural setting, the individual life-course and developed in opposition to the female gender and in relation to hegemonic, subordinate and marginalised constructions of masculinities. Hegemonic masculinity is hence not a natural given, but needs to be proven consistently, re-earned and established by the subordination and domination of women and other men (Connell, 2014; Connell \& Messerschmidt, 2005). This is true not just for conceptions of masculinity in young and middle age but also applies to the development of older male identities. Particularly hegemonic masculinity is determined majorly by displaying one's ability to control oneself and others. However, it is precisely this ability which declines with older male age since not only virility, physical and mental power but also social status formerly gained by one's professional occupation are severely impeded by older age and retirement. It can, therefore, be assumed that older age and the socio-cultural views which link ageing to waning physical and mental powers threaten the hegemonic status of men who were used to be on top of the male hierarchy (Hearn \& Sandberg, 2009; Jackson, 2016; Thompson, 1994; Thompson \& Whearty, 2004). In my view, men are therefore as much, although in different ways, affected by the sociocultural perceptions of ageing.

Hence, research with a focus on the experiences of older men could complement and extend research on ageing by adding a valuable perspective. Yet, when compared to studies investigating the public perception and individual life-course narratives of women, explorations of male ageing and particularly of the life-course narratives and identity construction of older men, who used to belong to the class of hegemonic men, are underrepresented in ageing studies (Arber et al., 2003; Dolan, 2018; Feasey, 2011; Hamad, 2013; Jackson, 2016). Within this context, it is also noteworthy that much qualitative research in cultural gerontology not only focusses on gaining a better understanding of the experiences of older women but also that most investigations are conducted within the Anglo-American cultural setting. Including the experiences of older men in western regions other than the UK or North America could, however, garner relevant insights into how older men use the available socio-cultural frames for older age and masculinity to construct their 
older identity in relation to the cultural specificities indicative for their country of origin (Connell, 2005; Jackson, 2016; Thompson, 1994).

In cultural gerontology and adjunct disciplines such as gender, men's and media studies, what one perceives as objective reality is viewed as socially constructed. It follows that the socio-cultural views linked to both gender and older age in the western hemisphere are neither natural nor unalterable but, in fact, they are shaped by and an outcome of the cultural and historical development in any respective society at any respective period of time (Edley \& Wetherell, 1995) (Connell, 1993; Connell, 2005). Media have since their advent exerted a high influence on the development and maintenance of socio-cultural values and standards and their power in today's more and more globalised world cannot be overestimated (Horkheimer \& Adorno, 2001; Wood, 1994) As such, mass media products must be viewed as a leading agent also in the dissemination and maintenance of socio-cultural narratives linked to gender, and older age and their products are resultantly progressively in the focus of research on ageing and gender (Dolan, 2018; Dolan \& Tincknell, 2012; Krainitzki, 2014; Swinnen \& Stotesbury, 2012).

Within the media landscape, Hollywood is still the dominating force in the worldwide film industry, and its products reach and are consumed by audiences around the globe. As a standardised mass product films produced in Hollywood, therefore, play a significant role in the transmission and solidification of socially acceptable role models, or in other words, in the construction and preservation of current hegemonic socio-cultural narratives of the occidental world (Miller, Govil, McMurria, Maxwell, \& Wang, 2005; Schatz \& Perren, 2004). Much of the recent scholarly debate of the construction of male role models in gender and men's studies consequently centres on the representation of masculinity in Hollywood mainstream films. A major part of these analyses investigates the portrayal of younger and middle-aged males which follows logically from Hollywood's focus on dominantly portraying youth and middle-age characters and its obsession with youth and youthfulness (Gabbard \& Luhr, 2008; Gallagher, 2006; Greven, 2010; Shary, 2013).

The relatively recent occurrence of older male characters in so-called geri-action films (Tucker, 2014) and what socio-cultural narratives their portrayal might be based on has, to date, received comparably little academic attention (Boyle \& Brayton, 2012; Lennard, 2014; Spector-Mersel, 2006). This is astonishing given the high commercial success of these films (Mojo Box Office, 2010, 2012, 2014) despite presenting audiences with action heroes well beyond their prime - something which neither seems to follow genre conventions nor be in line with Hollywood's celebration of youthfulness and related attributes such as good looks, productivity, autonomy and consumption power (Dolan, 2018; Lennard, 2014). A closer look at how the older male characters in films such The Expendables (Stallone, 2010), The 
Expendables 2 (West, 2012) and The Expendables 3 (Hughes, 2014) are contextualised so that audiences around the world accept and, indeed embrace these rather unlikely action heroes could, therefore, generate relevant insights into the construction of socially sanctioned scripts for older masculinities in the western hemisphere.

Simultaneously a discussion of media representations of older age in the west is a relatively common approach when critically examining the culturally and historically based concepts of ageing (Featherstone \& Wernick, 1995; Kotter-Gruhn \& Hess, 2012; Lyons, 2009; Thornton, 2002). However, these discussions are often characterised by an explicit focus on the contextualisation of female ageing. Male ageing and the analysis of the hegemonic narratives of masculinity and older age called on for the contextualisation of older male heroes have been less in the focus of academic analyses of media products (Arber et al., 2003; Featherstone \& Wernick, 1995; Nilsson, Hagberg, \& Grassman, 2013).

The aim of the dissertation is therefore to extend the insights gained in cultural gerontology and men's studies by developing a critical understanding of the hegemonic socio-cultural narratives of ageing and masculinity transmitted in popular cultural products to subsequently explore how older German men use these narratives to construct their identity and develop their life-course narratives in older age within a German cultural setting.

My research aims are, thus:

1. To explore dominant western socio-cultural narratives of ageing and masculinity

2. To examine the socio-cultural narratives at work in the portrayal of older men in the films The Expendables, The Expendables 2 and The Expendables 3

3. To explore how retired German men construct life-course narratives within and in relation to the socio-cultural narratives of older men and masculinity transmitted in popular cultural products of the occidental world such as in the films The Expendables, The Expendables 2 and The Expendables 3

\subsection{Assumptions, Limitations and Structure of the Study}

Since my research is informed by a social constructionist view of the world, it is based on a number of assumptions which have determined my research aims as well as my research design. I believe that the seemingly objective social reality everybody is part of is, in fact, humanly constructed and the result of historical developments. This implies that what is considered to represent the unquestionable and natural truth in any one society at any particular point in time is highly subjective and thus changeable (Berger \& Luckmann, 1991; Burr, 2006). Within this context, life-course narratives play a crucial role as they, to my mind, represent individual negotiations and adaptations of dominant understandings of 
reality and as such mark the starting point for changes in the socio-cultural understandings of social phenomena (Andrews, Squire, \& Tamboukou, 2008; de Medeiros, 2016; McAdams \& McLean, 2013).

In other words, I am convinced that the belief system of one's cultural context and linked narratives exert an enormous influence on individual constructions of life-course narratives within this particular socio-cultural context. At the same time, however, in my view, hegemonic narratives are adapted and altered to enable a representation as close as possible to reflecting individual experiences, views and values (Foucault, 1978, 2003; Foucault, Davidson, \& Burchell, 2008; Lorey, Ludwig, \& Sonderegger, 2016). These individual adaptations of hegemonic narratives then, to my mind, have the potential to produce some changes in dominant understandings of social phenomena transmitted as reflecting the objective truth. Such personal life course narratives are not only a powerful means for understanding individual constructions of identity in a particular culture better, but they also have the potential to enable social change by allowing access to differentiated and varied perspectives on identity constituting attributes such as gender and/or age.

My preference for qualitative rather than quantitative research approaches follows from my above-described research philosophy (Guba \& Lincoln, 1994, 2005). It forms the basis for my belief in the high significance of individual perspectives and for my scepticism towards research based on quantitative approaches in the context in which I am researching. The high significance I attribute to life-course narratives and their centrality in every individual's life in the west leads to further closely linked assumptions. First of all, I have based my investigation on the belief that human character and behaviour are mostly the product of their socialisation, their culture and of the experiences they make and the life they live and not of natural predetermination. Moreover, I assume that, at least, individuals living in western countries feel the strong need to create a consistent and stable identity which unifies the ambiguities and contradictions which characterise each person's life to form a coherent narrative (McAdams, 2015; McAdams \& McLean, 2013; Žižek, 2008). The resulting lifecourse narratives are an essential basis for a positive self-perception but are fully effective only when one shares them with others (McAdams, 2015). This leads me to yet another assumption, namely that human beings in industrialised countries are first and foremost social beings. Most people in the western hemisphere thus consistently seek the approval, recognition and appreciation of other human beings. Only through the feedback received one can feel significant and can develop a positive self-concept whilst a lack of social recognition or social disapproval can potentially have detrimental consequences for the individual (Laceulle \& Baars, 2014). 
I cannot exclude the possibility that my own belief and value system as a middle-aged German woman has affected the collection and interpretation of the data the selected films and my interviewees provided me with (Denzin, 2001; Hammersley, 2007). In other words, I cannot entirely rule out that I, without being aware of this, somehow led my interviewees in a particular direction with the questions I asked and that $I$ in the film and interview analyses interpreted the data so that they fit to predetermined believes of mine and confirm the assumptions my life-course narrative is based on. I have, however, tried to minimise this danger by staying as open-minded and reflective and as close to the data as possible (Denzin, 2001; Guillemin \& Gillam, 2004; Haraway, 1988; Koro-Ljungberg, 2008).

This thesis is also based on the assumptions that gender and sexual differences and linked attributes are socially constructed (Butler, 2011; Fausto-Sterling, 2000; Lorber, 1993), that constructions of masculinity and femininity are highly relational and depend on each other and that at any point in time several constructs of masculinity which are hierarchically structured exist at the same time (Connell, 2005; Kimmel, 2001). On the other hand, I view older age and related physical and mental changes as natural and as biologically determined while socio-cultural evaluations of older age are the outcome of the cultural system one lives in (Katz, 2015; Tulle, 2015). As such, I believe that as hard as one might try, it is impossible to, in the long run, escape the embodiments of older age. That physical and mental abilities decline with older age is a natural process but viewing slowness, weakness, dependency and frailty as something negative which seriously impedes one's social value and resultantly one's self-perception is, to my mind, an entirely human and socio-culturally produced conception (Gullette Morganroth, 2004, 2011). Nevertheless, there is always the danger in research such as mine to reproduce and affirm the hegemonic narratives one intended to explore critically (Baars, 2013; van Dyk, 2014). To prevent this I have attempted to provide the reader with as much detail and depth as possible to enable the contextualisation of my interviewees' remarks, thoughts and opinions.

This project is also based on the assumption that products of popular culture transmit sociocultural views hegemonic in a particular cultural setting and at a particular point in history. At the same time, I believe that, as a result of the increasing globalisation and the dominance of cultural products from the U.S. in cultures around the globe, these products and the narratives linked to masculinity and older age they disseminate, affect individuals in other and mainly western cultural contexts (Allen et al., 2015; Dolan, 2018; Dyer, 2013; Milestone \& Meyer, 2012). As such, this thesis is dedicated to an analysis of the inextricable link between current hegemonic understanding of ageing and masculinity and neoliberalism whilst also reflecting on the specificities of the German culture. 
Lastly, the analysis of just three films of a particular genre and produced for the mass market is somewhat limited in scope when considering that there is an increasing number of international films revolving around older age (DeFalco, 2016; Dolan, 2018). As such, the narratives explored in the film analysis could have certainly be extended by including films of different genres and independent and/or local, for instance, German, films. This could have increased the depth of this thesis, but it would have also markedly extended and changed its content which is the reason why I decided against it.

To investigate my research aims, I have structured my thesis as follows. I understand the literature review of scholarly work in cultural gerontology, and men's studies, the film analysis and the exploration of the four interviews conducted not only to be of equal significance but also as mutually informing each other. In other words, I perceive the conceptual framework identified through the literature review as an essential, inseparable and interdependent part of my empirical analysis. I have therefore decided to, after this introduction, begin my investigation with a focus on my methodological choices and the research design as I think it is important that I set out the epistemological and ontological platform for this project and make the basis for the decisions about the research design clear as they informed the ways I conducted the literature review, film analysis and interviews.

This is followed by a review of relevant literature in cultural gerontology and in men's studies which I separated into two chapters. In the first chapter, both key concepts underlying neoliberal master narratives of ageing and the effect of these principles on the identity construction of older individuals in neoliberal ideological systems are discussed. The second chapter of the literature review is dedicated to an exploration of key concepts in the constructions of masculinity. Within this context, I was particularly interested in highlighting commonalities in the conception of masculinities throughout history and particularly in Germany. The last section of chapter four revolves around gaining a better understanding of the impact older age has on public and individual perceptions of masculinity.

In chapter five, I analysed the geri-action film trilogy The Expendables to identify and by doing so illustrate which of the previously discussed narratives of ageing and masculinity are at work in these films. This aided me not only in better understanding constructions of masculinity and ageing and their transmission but also enabled further refinement of the core themes linked to ageing and masculinity I had established in the literature review and discovered during the interviewing stage of this project. After reflecting on the process and the challenges involved in the preparation, execution and analysis of the four interviews which are at the heart of this project in chapter six, I explored the accounts of my interviewees in relation to the key aspects discussed in the previous chapters. In chapter eight, the last chapter, I first summarised the main insights on the construction of older male 
identity within and around the socio-cultural narratives linked to ageing and masculinity I had gained in the course of writing this thesis with a particular focus on the four interviews I had conducted. This is then followed by a reflection on my personal development and the realisations I had arrived at as a result of the different stages involved in this PhD. Lastly, I indicated other avenues of research which, when pursued, could further extend and deepen an understanding of constructions of older age on a societal as well as individual level. 


\section{Methodology}

\subsection{On Becoming a Social Constructionist}

I embarked on the exciting adventure of my $\mathrm{PhD}$ since I wanted to extend my horizon and challenge myself to further my personal and professional development (Argyris, 2002). I was not disappointed. Seldomly have I learned as much in a relatively short time as during my $\mathrm{PhD}$ studies and rarely has anything I did, led to such ground-breaking changes in the way I view myself and the socio-cultural environment surrounding me (Argyris \& Schön, 1996).

When I began my studies, I believed to be all but a typical German. I had felt and still often feel somehow as an alien in my own country since I can and could not relate to the general German emphasis on order and rationality paired with displaying a strange insecurity and aloofness in social contexts which frequently leads to an either painfully shy or embarrassingly loud behaviour particularly when on holiday (Max-Planck-Gesellschaft, 2014). In fact, when I met people abroad who believed me to be another nationality than German, I was overly pleased - and I actually feel that way until today. In contrast, in a Mediterranean context, I felt like a fish in the water. Notwithstanding this, it was actually during my 7-year stay in Malta that I first realised that I might be more German than I had anticipated. Despite the fact that I was well integrated and rarely felt as socially accepted as in Malta, I was also often benevolently mocked for my very German punctuality, my obsessive need to plan social activities well ahead and my very, some might say overly, structured approach towards professional projects (Cramer, 2015; Wierzbicka, 2015). Apparently, I was more German than I had thought.

Indeed, my return to Germany was majorly a result of my own cultural embeddedness since I experienced an increasing need for security and a socio-economically stable system protecting its citizens from harm (Cramer, 2015; Max-Planck-Gesellschaft, 2014). I realised that foreigners, regardless of how warmly one was welcomed and of how easily one became part of the local community, would understandably never be fully integrated and accepted as a citizen with equal rights - after all, every country first and foremost cares for its own if they do at all (Esipova, Ray, \& Pugliese, 2017). Particularly my Mom's older age and her deteriorating health but also images of my own older age in Malta motivated the return to our home country in which we were entitled and could rightfully claim social benefits of a welfare system ensuring necessary levels of economic stability and physical and mental well-being (Max-Planck-Gesellschaft, 2014; Zimmer \& Toepler, 1996).

Nonetheless, when I returned back to Germany ten years ago, I found working and living in Germany very difficult. The strict division between work and private life, the obsession with 
never losing composure and with always keeping in control particularly by never under any circumstances admitting weakness, an error or, even worse, failure and the elitism especially in an academic contexts were just some aspects which I found alienating (Chang \& Aldred, 2014; Cramer, 2015; Wals \& Jickling, 2002; Wierzbicka, 2015). As a result and not surprisingly an international private university characterised by a broad international and cross-cultural workforce and student body became my new professional home, and my newfound circle of friends either came from an Anglo-American background or were Germans who had lived abroad for a considerable time.

When I started with the PhD programme, I was well aware of the challenges ahead of me but relatively confident that given my experience with British vocational certificate courses these challenges would not lie in getting to grips with cultural differences in educational approaches but more in the academic expectations. Initially, this assessment seemed to prove right since I had much fewer problems with the interactive and mentoring teaching style than many of the other participants in the course who had expected to be told what to do and how to do it rather than to be guided towards discovering effective ways of professional and academic development on their own (Joy \& Kolb, 2009; Schön, 1983). However, when we started discussing research paradigms (Guba \& Lincoln, 2005), I was first astonished that I had neither been exposed to research philosophy during my university studies nor during my time as a lecturer for, among other subjects, scientific and academic working in Germany. Subsequently and once I had researched the area in more depth, I became extremely frustrated and felt quite lost not only since I could not really get my head around the various concepts but also since I was all than sure which research paradigm (Kuhn, 1970) would best reflect my own research position.

It was during this time that I started realising how German I really was. Although I despised the very German need for control and order, I wanted to follow an approach that allowed me to keep in full control of my research. I longed for a research paradigm providing me with clear guidelines for my project and precise procedures ensuring academic credibility of my thesis (Matzler \& Renzl, 2005; Schommer-Aikins \& Easter, 2006; Schommer, 1990). My original approach had already changed from a quantitative to a qualitative orientation, but I was still struggling with the lack of representativeness, related issues of validity and the, in my eyes, insignificant outcome of my qualitatively oriented research project (Hammersley, 2007). I know now that this understanding was primarily based on my German background. I grew up in a society in which rationality, objectivity and logic are seen as the basis for any 'proper' scientific work whilst subjectivity, emotions and disorder are viewed as the natural enemy of research (Schön, 1983). In addition, feedback on my planned research project from colleagues and friends at work and in my social circle of friends and acquaintances was at 
best ambiguous and at worst outright doubtful regarding the scholarly standard of my work and its scientific significance and validity (Chang \& Aldred, 2014; Kuhn, 1970). As a result of my Germanness and my low confidence in my own scholarly work, on the one hand, and, on the other hand, the belief that a qualitative method was the only suitable approach to reach my aim, I tried finding a middle way. I thus selected a philosophical stance which could provide me with control and clear procedures while allowing for a qualitative orientation of my project (Levsen, 2008; Matzler \& Renzl, 2005; Schön, 1983).

I had discussed and applied three potential research paradigms in the respective assignment, namely critical realism, phenomenology and critical theory. Although the significance attributed to capturing the specific lifeworlds of individuals in phenomenology mirrored my own view, I found it extremely difficult to subscribe to the postulate of becoming a disinterested observer during the research process by bracketing off everything which characterises existence in the life-world (Schütz, 1967, 1970). I could then and now not understand how becoming an indifferent observer by stripping off anything which links him or her to social reality to be able to collect individual accounts of reality is possible and why it is necessary. Thus, I excluded this research paradigm.

Both critical realism and critical theory seemed to reflect my understanding of reality better. My German soul was overjoyed by the structured and logical way which Roy Bhaskar (1975, 2009, 2013) had developed to uncover the mechanisms involved in the complex and stratified character of reality while conceding to the fact that reality is too complex ever to be fully understood by humans. At the same time, having grown up in a very liberal and leftwing oriented home, I naturally felt inclined to critical theory. Its specific focus on understanding the perspectives of underprivileged groups and, by doing so, on giving these groups a voice to enabling their empowerment and changes in their socio-cultural and politico-economic position, was, or so I thought, exactly what I wanted to do in my research (Comstock, 1982; Fay, 1987; Strydom, 2011).

However, as a result of German history, I, like many Germans, was and am sceptical of the high significance assigned to the emancipatory aims in critical theory. In my view, it contains an inherent danger of justifying disrespect for the well-being and the autonomy of individuals by facilitating a greater good, namely freedom, and resulting happiness, for all (Fay, 1987). German history is an impressive illustration of the catastrophic consequences of systems based on the view that humans need to be educated and made aware, if necessary by force, to see what is good for them (Fulbrook, 2004). Although the primary driver of national socialism and communism was, of course, to gain and ensure power, both dictatorships justified the repression and eradication of anybody who did not fit into the 
political system by the intention to facilitate a greater good which would eventually benefit all members of society (Fay, 1987; Steiner, 2013).

By the end of the assignment I had, therefore, come to the conclusion that: " On the one hand, I strongly relate to most of the ontological and epistemological presuppositions constituting the basis for critical realism. On the other hand,..., I feel affiliated with many of the assumptions and methodological choices critical theory is based on". Having received an A on my assignment, I was not only extremely pleased with myself but also convinced that I had finally found my research stance. All the more was I shocked and taken aback when one of my tutors told me that this approach was not in the least unusual for a German and that she was quite sure that my position would change during the development of my project - and she was right.

The further I proceeded with my $\mathrm{PhD}$, the more I caught myself being drawn to literature based on a social constructionist research paradigm (Berger \& Luckmann, 1991; Guba \& Lincoln, 1994, 2005). This was corroborated by the feedback I received from my supervisor and her reading suggestions. Nevertheless, I was still ambiguous and somewhat reluctant to let go of the control inherent in the worldview and procedures suggested by critical realism, and the emancipatory aims of critical theory since I continued struggling with the epistemological relativism social constructionism is based on (Burr, 2006; Lawson, 2003). If every individual's perspective was unique and equally valuable, how could my research of older and retired German men contribute something significant to academia? Or, in other words, what then would be the aim of my research? At this point, 'just' understanding individual perspectives seemed too little to me to justify my research and, yes, I admit, it also did not seem to be something which could gain me the much needed and wanted scholarly reputation.

Simultaneously, being brought up in the 90s, I, subconsciously, was the picture-perfect example of a postfeminist (Negra, Tasker, \& McRobbie, 2007). As such my views resembled the ambiguity characteristic of post feminist worldviews. I was grateful for the efforts of feminists whose work and political outcomes I benefitted from, but I also entertained a rather narrow and reductionist view of radical feminism. I perceived radical feminism's attempts to enable changes in gender hierarchies as unnecessary since I believed that overall women in Western countries were already emancipated and empowered and that the disadvantages they still faced were minor. I realised, however, that I had been blissfully unaware and had totally neglected "both evident economic disparities and the fact that the majority of women approach paid labour as an economic necessity rather than a "choice"'(Tasker \& Negra, 2007). 
I was also critical of the rather one-dimensional view of men as the 'enemy'. I believed and believe that both women and men are victims of patriarchy although in different ways and I am convinced that alterations to the gender hierarchy are only possible if women and men critically reflect on the aspects which determine the categorisation of oneself as male or female (Budgeon, 2014; Haraway, 2013). This is one of the reasons for my decision to focus on the construction of ageing masculinity rather than on the construction of ageing femininity in this thesis. Yet, contrary to what I presumed, the more I researched, the more I found that I shared this view with many feminists in academia (Baur \& Luedtke, 2008; Hamad, 2013; Negra \& Tasker, 2014; Saxton \& Cole, 2013; Spector-Mersel, 2006; Tasker \& Negra, 2007).

Engaging with scholarly texts exposing the socio-cultural and politico-economic structures patriarchy is based on (Bourdieu, 2001; Butler, 2011; Fausto-Sterling, 2008; Lorber, 1996) while working in a male-dominated faculty at a business university, has recently altered the way I see myself and my perception of the world around me. Work on my $\mathrm{PhD}$ has sensitised me to the mechanisms of gender hierarchy, and I became aware of the subtle and often, I believe, subconscious processes allowing patriarchy to continue thriving. In fact, I am no longer able to overlook the numerous, continuous and, taken separately, insignificant ways in which men ensure their dominance over women. Nor am I able to disregard the fact that women, including myself, make this possible by accepting patronising and belittling male behaviour and by strategically using so-called feminine weapons such as appearance, charm, sexiness or naivety to gain agency and control over men. By striving to fulfil gender norms, a pre-requisite for social acceptance, both men and women consistently re-enact the constituents of gender hierarchy and thereby prevent any systemic changes (Bourdieu, 2001; Butler, 2011).

So, after all, and despite being German, over the course of writing this thesis, I have not only developed into a full-fledged feminist but also into a social constructionist. Although I still believe that there is an objective reality, I am more than ever convinced that as a result of one's own socio-cultural embedment it is never possible to approach and research this reality objectively (Berger \& Luckmann, 1991; Haraway, 1988). Resultantly, reality can, in my view, only be approximated (Bhaskar, 1975, 2013). My work is, therefore grounded in the assumption that knowledge is always historically and culturally determined. It can hence only provide a snapshot of a specific perspective at a specific time in a specific cultural context. Knowledge is, thus always provisional and, in the double entendre of the word, partial or in Donna Haraway's words: “Feminist objectivity means quite simply situated knowledges" (1988, pp. 581, emphasis added in original). 
Nevertheless, examining various individual perspectives and uncovering the complex relationship between idiosyncratic views of reality and currently dominant socio-cultural and politico-economic structures is worthwhile. To my mind, it is the prerequisite for a better understanding of the highly intricate processes involved in the identity construction of individuals within the boundaries of a particular cultural setting and historical time (Doucet \& Mauthner, 2006; Harding, 1992). Within this research context, an explicit focus on capturing the perspective of marginalised groups is, in my view, especially relevant since one must first create awareness for the victims of the dominant ideology to enable societal changes in the current power structures (Haraway, 1988). This conviction is the outcome of my own development described above: Only my engagement with various accounts of reality and subsequently gaining a better understanding of individual viewpoints permitted me to change my own worldview.

In other words, combining ontological realism with the epistemological relativism advocated in feminist standpoint philosophy (Bhaskar, 2009, 2013; Lawson, 1999), allows me to focus on marginalised groups and on uncovering their worldviews without losing myself aimlessly in a relativistic universe in which all subjective accounts of reality are equally significant, valid and real (Elder-Vass, 2012; Haraway, 1988). Instead, I now understand my research as a relevant, even if tiny, contribution to knowledge for two reasons. Firstly, I think that lending a voice to people who are far removed from hegemony is essential in perforating the hegemonic privilege of determining which accounts of reality and which knowledges are sanctioned as representing the 'truth' and which aspects of this 'truth' are subsequently constructed as representing facts defined by nature and hence unchangeable (Bourdieu, 2001; Fay, 1987; Foucault, 2000). Only the inclusion of marginalised perspectives can facilitate a more differentiated and deeper understanding of the socio-cultural reality in a particular place and time (Berger \& Luckmann, 1991; Haraway, 2013). At the same time, placing emphasis on groups of people who are at the side-lines of society will subsequently create more visibility for their wants and needs (Harding, 1992). This is, in my view, the prerequisite for societal changes enabling a sustainable society which is inclusive rather than exclusive.

\subsection{Research Design}

The selection of a qualitative research design is a logical outcome of my research position and my research aims. As discussed above, I believe that reality is constructed through individual behaviour and human interaction and, at the same time, through a societal apparatus consisting of the legal system, educational institutions and science, health 
organisations and medicine, and the media, to mention but a few. Since the micro-and macro level in occidental societies, therefore, inform each other and are inextricably intertwined (Foucault et al., 2008) I have opted for a research design which permits exploring both dimensions and their complex relationship (Burr, 2006).

My interest does, however, not lie in explaining phenomena linked to male ageing by gathering a high amount of data enabling statistical examination based on the comparability, representativeness and breadth of the collected data (Grix, 2010). Instead, my project is grounded in the strong desire to facilitate a better understanding of the life-course narratives of older German men constructed within, outside or in opposition to historically bound narratives of ageing and masculinity informing their socio-cultural environment (McAdams \& McLean, 2013; Weedon, 2004). Following a qualitative approach which enables the collection of rich data and thereby supports sufficient depth in the exploration of the accounts of my interviewees as well as in the film analysis, is, therefore, in my view, a coherent and obvious choice (Geertz, 2008; Merriam, 2009; Patton, 2014).

My preference for qualitative methods is further corroborated by my scepticism towards the use of approaches gathering purely empirically visible data. In my view and particularly within my research context social processes are much too complex to be approached merely by accumulating observable evidence (Danermark, Ekstrom, \& Jakobsen, 2005; Maxwell, 2012; Strydom, 2011). I believe that to gain insight into the inner world of individuals, the highly intricate outside social world and the even more complex interplay between the two, one needs to move beyond visible and exclusively exterior examinations. Within this context, language is of high significance since individual, as well as socio-cultural narratives, are predominantly constructed and develop further in and through the communication and interaction with others (McAdams, 2015; McAdams \& McLean, 2013; Rose, 2016). In other words, every text produced and communicated by individuals as well as by the cultural communities each individual is part of carries a literal or obvious and a connoted and contextually dependent meaning. I am interested in the former, the signifier, as well as the latter, the signified, since both are intrinsically tied to each other and resultantly play a significant role in personal and social meaning-making processes (Rose, 2016; Žižek, 2006, 2008).

Given that exploring the significance of cultural narratives of masculinity and ageing are at the core of my research, only a qualitative research design is, in my view, feasible. To facilitate answering my research questions, I have, therefore, chosen to follow a three-part qualitative research design in which the insights derived at any one part mutually inform the other parts (Patton, 2014). A review of relevant literature in cultural gerontology and men's studies has provided me with a tentative and provisional conceptual framework for both, the 
initial analysis of the action film trilogy The Expendables and the semi-structured interviews with four older German men.

Although combining film analyses with semi-structured interviews is an unconventional approach, I decided to follow this approach despite the doubts my supervisors and I had at different stages of my research with regards to the benefits of the film analyses for reaching the aims of this thesis. In hindsight, I believe that this decision indeed enabled deeper insights into the socio-cultural constructions of masculinity and ageing. Simultaneously working on the film analyses and the literature review aided me in better understanding how the often quite abstract key narratives of successful ageing and ageing as decline and masculinity discussed in the literature are mapped onto products of popular culture. At the same time, the in-depth analysis of narratives of ageing and masculinity disseminated and accentuated in The Expendables trilogy permitted me to reflect on the need to elaborate on certain aspects which I had either dismissed or discussed in little depth in the literature review and on the overall cogency of the themes discovered in scholarly discussions. Subsequently, the desire to gain more insight into how older German men use the socio-cultural frames of ageing and masculinity found in the literature review and the film analysis informed the questions asked in the interviews and the interview analysis while the flexibility of my research design permitted me to go back to the literature review respectively the film analyses and adapt, extend and refine the themes found on the basis of what my interviewees told me and which aspects related to ageing and masculinity they emphasised.

The highly iterative research approach in which I continuously compared the outcomes of the literature review with the results of the film analysis and the interview data facilitated sufficient depth in the analysis since it aided me in adapting, extending and refining the initial themes (Braun \& Clarke, 2006). This allowed me to explore a deeper understanding of the interface between the cultural narratives conveyed in the chosen films, the experience of older German participants and the socio-cultural context that they operate in.

Popular cultural products play a significant role in the cultivation and maintenance of socially acceptable role models since they reflect and simultaneously transmit dominant cultural narratives (Katz, Blumler, \& Gurevitch, 1973; Richardson, 2019; Schweinitz, 2011). As a result of technological advances in computer technology and the increasing globalisation in current western societies, mass media have not only changed but also diversified and extended the channels available for the dissemination of their content. Consequently, their influence as socio-cultural agents of hegemonic socio-cultural standards and values are today as powerful as ever (Machin \& Van Leeuwen, 2007). Despite the emergence of new media outlets, such as social media, old-school feature films and particularly Hollywood blockbusters have not lost their appeal for the broad mass of 
audiences (Miller et al., 2005; Schatz \& Perren, 2004). Within this context, the appearance and rising popularity of action films whose cast consists of older action stars between sixty and seventy, so-called geri-action films (Donnar, 2016), is not only the outcome of, as some believe (Hulls; Tucker, 2014), a shortage of capable younger action stars, but also undoubtedly a reaction to and an indication for an increasing public awareness for the dramatic demographic changes underway in industrialised countries (Hulls; Orr, 2010; Singer, 2014; Smith, 2010; Tucker, 2014). Within the context of this thesis, these films are of particular interest since the genre of action films is considered " [...] the most irredeemably of macho Hollywood products [...]" (Tasker, 1993a, p. 242). As such action films in general seem to be an apt starting point for gaining a better understanding of cultural narratives linked to masculinity. Exploring the narratives employed in the portrayal of older men in geri-action films should additionally provide insights into the potential adaptations and changes of masculinity scripts related to the increasing age of men.

To examine the cultural narratives at work in the portrayal of older men in geri-action films, I have selected to analyse The Expendables, The Expendables 2 and The Expendables 3. I believe these Hollywood mainstream action films to be especially suitable for analysis as they were produced for the commercial mass movie market and therefore rely strongly on formulaic narration and characterisation (Tasker, 2004). Moreover, since the majority of the main characters in both films are between their mid-forties and mid-sixties (Tucker, 2014), it can be assumed that their depiction contextualises and reflects dominant respectively socially accepted cultural narratives associated with older men. This assumption is not only supported by the high domestic and particularly foreign gross earnings of the selected films but also by the fact that the first film was followed by the production of two sequels (Mojo Box Office, 2010, 2012, 2014). To my mind, this serves as additional proof for the supposition that the Expendables trilogy successfully captures contemporary cultural narratives linked to ageing and masculinity (Schatz \& Perren, 2004). In addition and despite the fact that each of the three films is a self-contained product of its own right and understandable without needing to see the other two films, all three films are thematically similar, and the main cast and the storyline predominantly remain the same. This enables a comparison of the thematic units discovered. In other words, the results of the film analyses can be cross-checked within and outside the case in focus by examining the thematic units identified in each film alongside the themes found in the literature review and in the interview analyses. Hence, the depth of the analysis offered in this thesis is enhanced without sacrificing the manageability of the data (Yin, 2013).

To explore how retired German men construct life-course narratives within and in relation to the narratives of older men and masculinity identified in the films The Expendables, The 
Expendables 2 and The Expendables 3 and in the literature review, I decided to conduct interviews with four German men. I considered interviewing the most suitable method available since it permits "us to enter into the other person's perspective"(Patton, 2014, p. 426). As such, it is one of the few research methods allowing some access to the inner world of individuals (Patton, 2014). I would have liked to use an informal and unstructured way of interviewing only based on an interview guide consisting of thematic units since it permits the highest level of interaction, flexibility and involvement of both the interviewees and the interviewer and it, therefore, most closely fits my research position and aim (Flick, 2009; Patton, 2014). However, due to my inexperience in interviewing and my German need to retain some control over the research process, I decided for the next best alternative - a semistructured approach to interviewing. The initial open-ended questions were the outcome of my literature review. Based on my reflections on the pilot interview as well as the subsequent interviewing process these were then continuously adapted, changed and excluded when I felt that these alterations could increase the depth and richness of the data (see section 6 for more detail) (Grix, 2010). Depth was further facilitated by asking follow-up questions to enable a better understanding of the aspect discussed by the respective participant and to clarify and consolidate the individual perspectives (Patton, 2014; Yin, 2013).

The interviews were conducted with four retired German men considered within the German context to be of an upper-middle-class socio-economic background. I viewed the socioeconomic status of my participants as crucial since it, on the one hand, indicates that they have successfully adapted themselves to the current hegemonic socio-cultural system while, on the other hand, it firmly positions them in the inner circle of the privileged men and occasional women who are most powerful in a neoliberal society (Bourdieu, 2001). Their retirement from professional life marks an important caesura in their life since older age and being a pensioner catapult older men from being members of a dominant group to being part of a marginalised group even if they retain the lifelong privilege of being a man (Baur \& Luedtke, 2008). These changes in status and related self-conception necessitates alterations in life-style, personal identity and life-course narratives (Gullette Morganroth, 2004; Jackson, 2016). How older German men managed the transition from employment to retirement and from being representatives of hegemonic masculinity to being placed on the side-lines of society is precisely what interested me and what I wanted to find out more about. These aspects, therefore, motivated the selection of my interviewees and determined the questions I asked them.

The analyses of the films and the interviews are based on viewing texts as revealing and at the same time, consolidating power structures and hierarchies (Rose, 2016). As such my analytical method is oriented towards the discourse-historical approach which aims at " $[\ldots]$ 
de-mystifying ideologies and power through the systematic and retroductable [sic!] investigation of semiotic data (written, spoken or visual).”(Wodak \& Meyer, 2009, p. 3) As suggested by the representatives of the discourse-historical critical analysis (Barker, 2008; Wodak \& Meyer, 2009), I developed thematic units which were guided by the research questions and informed by the reflection on and synthesis of scholarly work crucial for the discussion and better understanding of the historical development of hegemonic sociocultural narratives of masculinity and ageing. These thematic units were then further refined and adapted by moving continuously between the findings from the literature review and the empirical data (Wodak \& Meyer, 2009). This procedure supported a continuous process of reflexivity and provided me with the freedom to adjust themes developed in the course of investigation (Denzin, 2006). However, as perfectly as the discourse-historical text analysis supported the initial exploration of narratives linked to ageing and masculinity in the Expendables trilogy, it did not prove to be quite as useful in the interpretation of the interviews I conducted.

When reflecting on the results of my interviews, it was of utter importance to me to develop a framework that allowed me to gain an understanding of the life-worlds of my participants as holistically and untainted by my own preconceptions as possible (DiCicco-Bloom \& Crabtree, 2006; Robertson \& Hale, 2011). In my view, only aiming at a documentation of their accounts as thick (Geertz, 2008) and non-judgemental as possible would pay the appropriate level of respect to my interviewees and to the trust they had shown me by talking about their lives and feelings so extensively and openly. I did not under any circumstances want to fall into the trap of standing on the high grounds of academic elitism, assuming to know more about the workings of society and individual identity construction than my participants. This can, I believe, only lead to making the data fit my presumed knowledge instead of adjusting and extending my views of the world by processing what I had learned from my interviewees (Haraway, 1988). It was, therefore, of utmost importance to me to continuously reflect on the interview questions and their adequacy in capturing the individual perspectives of my interviewees and subsequently on my understanding of the data. To support this approach, I reflected on the interview process after each interview and the effectiveness of the questions asked in a diary. During the analysis of the interviews, I consistently questioned the accuracy of my interpretation of the data in an inner dialogue and at times, conferred with other researchers to compare our understanding of the respective data. This aided me in keeping an open mind and in being as reflexive as possible about my own socio-cultural embeddedness as possible (see chapter 6 for further details) (Denzin, 2001). 
However, the explicit focus on exposing power structures in the discourse-historical approach to text analysis leaves little room for viewing the data neutrally and holistically since it determines the perspective of the researcher and what he or she should be paying close attention to before even starting to engage with the data (Wodak \& Meyer, 2009). With this in mind, I used the themes identified in the literature review and the film analyses as a guide for a first and rather basic categorisation of the responses of my participants, to then apply a highly intuitive approach in which I let the data speak for themselves (Braun \& Clarke, 2006). This allowed me to expand and deepen my insights into older men's experiences with living life as a man, with becoming an older man and with the chances and challenges this involved (Jackson, 2016).

Immersing myself in the testimonies of my participants in the described way was intended to allow me and the readers genuine and novel views on older men's inner worlds s to create more awareness for the specificities of their life-course narratives and self-perception within the context of ageing and the multitude of aspects involved in getting older as a man in Germany (Andrews et al., 2008; Brockmeier \& Carbaugh, 2001). Subsequently, I hope that my interview analysis will expand and enhance the current understanding of ageing in the west which, in turn, could facilitate a revision of the societal depreciation of older age by moving the attention paid to older people from the periphery more into the centre of societal concern and significance.

To increase the transparency, authenticity and readability of both my film and interview analysis, I stayed very closely to the data and used plenty of direct citations from the films as well as from the interviews to support and illustrate my claims while staying as truthful to the data as possible (Grix, 2010). To enable a deeper exploration and resultantly thicker description of the interviews (Geertz, 2008) I first reviewed the responses of my participants to assess how far the initial codes developed during the previous stages of my project could be used to categorise the interview results (Braun \& Clarke, 2006).

Although overall the initial codes proved useful, I nevertheless refined, altered and summarised some themes to enable a better fit between the data and the codes (see chapter 6 for further detail). This was followed by sifting through each interview transcription repeatedly to identify aspects mentioned by the participants in connection to the main themes. I wanted to find out which elements within the context of the main themes were mentioned, and therefore relevant and significant for my interviewees. This process was supported by tabularising the answers of each participant to each question so that I could better compare similarities and differences in the responses of my interviewees and ensure that I had covered all varieties in their utterances (Cullum-Swan \& Manning, 1994). To visualise the different dimensions of each theme, I devised a mind map for each main topic 
detailing aspects discussed in relation to the respective theme. I have included the mind maps in the appendix to illustrate the range of aspects linked to each main thematic unit (see appendix K-P). In the last step, I went through each interview transcription again to ensure thoroughness, completeness and inclusivity of the narrative analysis (Braun \& Clarke, 2006; Cullum-Swan \& Manning, 1994; McAdams \& McLean, 2013; Weedon, 2004). Below is an illustration of my overall research design, while a detailed description of and reflection on the different stages involved in the interview analysis can be found in chapter 6 of this thesis. 


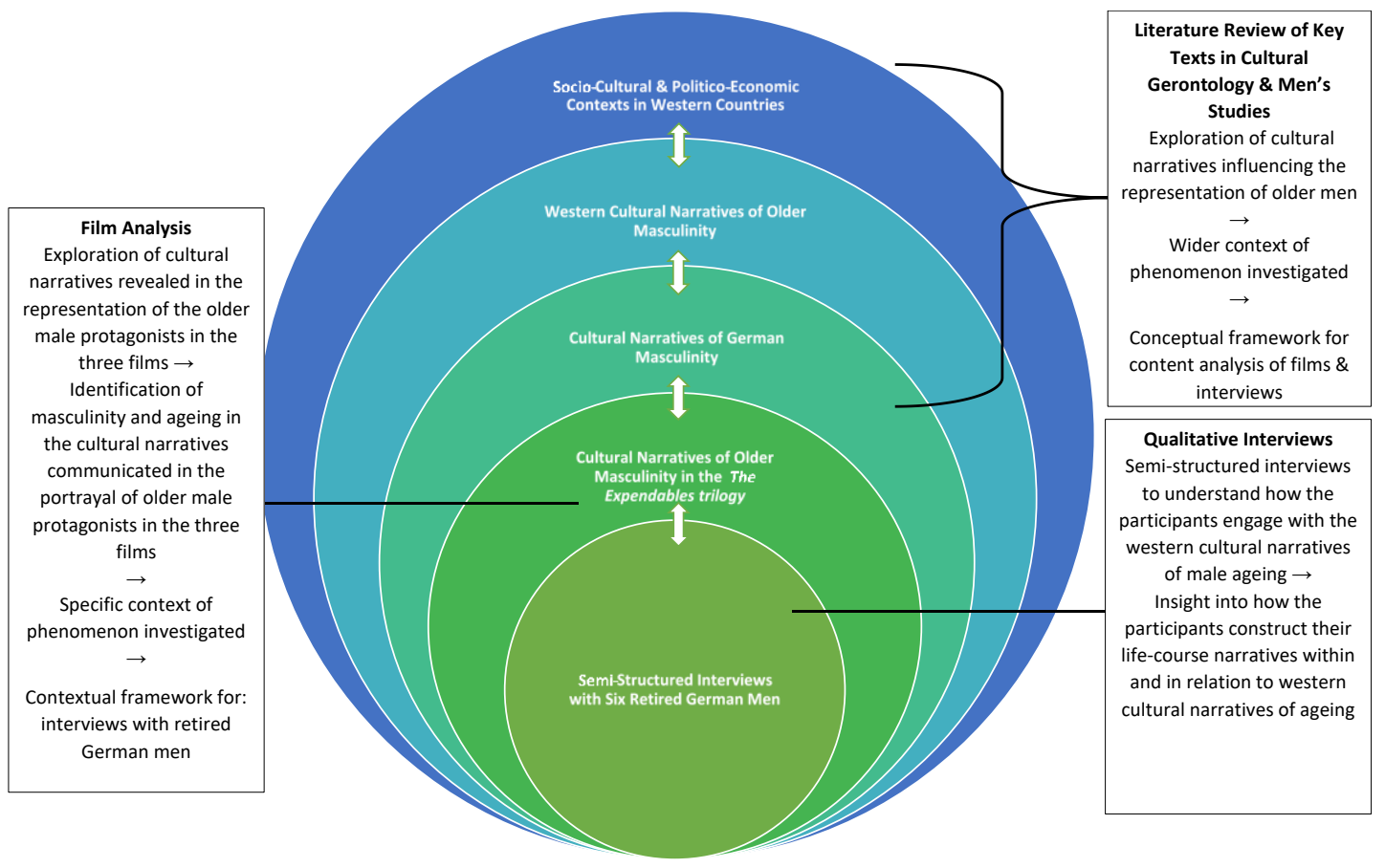

\section{The Construction of Ageing in Neoliberal Societies}

\subsection{Neoliberal Master Narratives \& Individual Identity Constructions}

As highlighted above, social constructionism is based on the understanding that social structures are grounded in historical processes and human-made. The embeddedness of societal structures in culture is supported by their dissemination from one generation to the next and its codification and solidification in the socio-cultural, legal and governmental rules and regulations of a society (Chomsky, 1999; Fay, 1987). As a result, the humanly constructed, established and maintained mechanisms of society are objectified and perceived as unchangeable laws which are part of an objective reality determined by nature (Bourdieu, 1990; Chomsky, 1999; Harvey, 2007a; Horkheimer \& Adorno, 2002).

However, as proven by history, the rules governing human activity can be changed by humans if uncovered, critically investigated and questioned. Indeed, human agency, sociocultural standards and the seemingly unchangeable and objective social reality are inextricably linked to each other (Collier, 1994; Fay, 1987; Foucault, 2000; Foucault et al., 2008). Hence, as afore-mentioned, I believe that to understand, explain and potentially change social phenomena it is necessary to examine both, the mechanisms guiding social phenomena and the subjective viewpoints of the group influenced by these social processes. 
Within this context, the significance of master narratives in the transmission of socio-cultural standards and values and their impact on individual identity constructions cannot be overestimated since they are essential for the establishment and maintenance of core societal values and rules and every member of a given society grows up with (de Medeiros, 2005, 2016; Laceulle \& Baars, 2014). They are passed on to us by our main social peer groups from the day we are born (Berger \& Luckmann, 1991; Bourdieu, 2018; Žižek, 2006, 2008) and their internalisation is further supported by socio-cultural agents such as the media, educational and cultural institutions and the law, surrounding us every day (Chivers, 2011; Featherstone, 1995; Horkheimer \& Adorno, 2001, 2002). Master narratives dominant in a specific socio-cultural setting at a particular time reveal the hegemonic touchstones of a society. Therefore gaining a better understanding of these narratives is of high relevance when exploring social phenomena (Donnar, 2016; Eckersley, 2006; Hall, 2000; Holmlund, 2010; Katz \& Calasanti, 2014; Phoenix \& Sparkes, 2006; Spector-Mersel, 2006; Tarrant, 2010).

Postmodern western society is characterised by the ever-increasing global dominance of neoliberalism (Chomsky, 1999; Harvey, 2007a). Neoliberal ideology significantly influences the ethical, social and behavioural benchmarks against which the individual is judged. Neoliberal master narratives determine how one defines success, failure, marginalisation and dominance (Hoffmann et al., 2015) and they therefore also exert a critical influence on societal and individual understandings of ageing and related processes as well as on constructions of gender (de Medeiros, 2016; Laceulle \& Baars, 2014; Richardson, 2019).

Neoliberalism is based on the view that markets freed from governmental regulation and interference are the prerequisite for economic development and the flourishing of countries. It consequently centres its activities on securing private property rights and market liberties by implementing suitable legal and governmental regulations whilst insisting on a minimum of market supervision by governments since governments, unlike economists and entrepreneurs, are not in a position to understand the complex market structure (Harvey, 2007b). Neoliberalism thus "seeks to bring all human action into the domain of the market" (Harvey, 2007a, p. 3). Accordingly, current neoliberal society rests on three main master narratives: consumption, production and individual autonomy (Bauman, 2013; Featherstone, 1991).

Firstly, self-fulfilment and the belief that consumption is the basis for it has established itself as one of the primary purposes of most people living in neoliberally oriented societies (Žižek, 1989, 2006). Self-realisation gained by consumption has developed into the neoliberal duty of the individual firmly inscribed into one's existence by the socio-cultural 
order one is part of. The constant preoccupation with it ensures that one neither has time to question the ideology underlying current societal values nor the energy to step out of the system which structures one's life (Chomsky, 1999; Harvey, 2007a; Žižek, 2008).

To be able to consume one needs the means for consumption - money. The counterpart of consumption is, therefore, productivity, a value deeply rooted in the western socio-cultural system since the establishment and increasing popularity of Protestantism in the $14^{\text {th }}$ century. The understanding that hard and continuous work is the prerequisite for salvation ties in particularly well with the constituents of capitalistic and post-capitalistic society (Weber, 2013). Master narratives on the lines of 'you can reach everything if you try hard enough' or 'the American dream' have at their core the belief that only hard work qualifies you to benefit from societal benefits (Gullette Morganroth, 2004; Gullette Morganroth, 2008). Only being and keeping busy and productive provides the individual with the justification to reward him- or herself through consumption (Gergen \& Gergen, 2000). Productivity must hence be viewed as the flip side of consumerism, and it is essential in ensuring the preservation of the politico-economic system of neoliberalism. It follows that the individual's productive value is determined by his or her employment and related income. This, in turn, has a crucial impact on one's societal status and on how one perceives oneself and is perceived by others. Thus, the individual's production value increasingly determines one's value for oneself and others (Price \& Livsey, 2015).

The holy trinity of neoliberal society is completed by its strong emphasis on individuality, autonomy and personal responsibility. By doing so neoliberal society places the responsibility to make the best out of one's life on the individual while at the same time restricting individual choices by the socio-cultural frames it provides (Carroll \& Bartlett, 2015; Laceulle \& Baars, 2014). The emphasis on individual freedom and the social dictate to find self-realisation through consumption while being productive members of society who aspire to become successful, influential and affluent at nearly all costs, secure that individuals do under no circumstances want to become a burden to society. Having to rely on social and medical services of the state or, even worse, becoming dependant on the help of relatives and friends has become a horror scenario for postmodern individuals in the west (Katz \& Calasanti, 2014; Katz \& Marshall, 2003). Inflating the significance of personal responsibility as a crucial criteria for evaluating one's life has led to an obsessive focus on staying independent since losing autonomy as a result, for instance, of the ageing process means losing societal privileges such as respect, influence, status and above all, perceived control which, in turn, makes one feel worthless, insignificant and useless (Laceulle \& Baars, 2014; van Dyk, 2014). 
Neoliberal master narratives of the west shape how individuals think and feel about themselves and, at the same time, they set the parameters for the evaluation of others (Nelson \& Lindemann, 2001; Richardson, 2016). In the fierce competition for societal privileges and power, one's self-construction in industrialised countries of the west fundamentally rests on the "commodification of the self-identity" (McDonald, Gough, Wearing, \& Deville, 2017, p. 367). Whilst in former times religious or political belief systems could serve as a crucial point of reference for one's identity constructions, today individuals in the west have, except the above-discussed market values of autonomy, productivity and consumption, little to base their identity construction on (Chomsky, 1999; Žižek, 2006, 2008).

In current neoliberal society, however, one seems to question precisely these values increasingly and to refuse indoctrination (Žižek, 1992, 2006, 2008). The critical and often cynical attitude towards neoliberal ideology and linked belief system leaves the individual, in our increasingly complex consumer society with its myriad of choices to be made every day, rather unoriented (McDonald et al., 2017). It is, therefore, no surprise that although the post-ideological individual does not believe in the authority of the politico-economic system underlying society and yet continues to fulfil his or her roles in society tirelessly:

[T] hey know very well how things really are, but still they are doing it as if they did not know ...For example, they know that their idea of Freedom [sic!] is masking a particular form of exploitation, but still they continue to follow this idea of Freedom [sic!]( (Žižek, 2008, p. 30).

As a result, the individual scepticism towards core neoliberal master narratives does not translate into a discussion of alternative socio-political systems but exhausts itself in fighting for the interests of marginalised groups and their rights within the boundaries of neoliberal socio-cultural frames we are, for a lack of alternatives, unable to let go of (Hall, 2000, 2015). Within this context, social comparison has become a crucial means for establishing one's and other people's 'market value'. 'As such, our obsessive need to evaluate everything and everybody must be viewed as an essential instrument in the construction of individual identity in the occidental world.

Leon Festinger's theory of social comparison (1954) assumes that upwards and downwards comparison aids us in judging our intricate social environment, finding our place in it and in developing identity (Corcoran, Crusius, \& Mussweiler, 2011; Festinger, 1954; McDonald et al., 2017). Social comparison hence fulfils a crucial function in the development and maintenance of a positive self-perception. It aids individuals in determining their position in neoliberal socio-economic systems. Downwards social comparison increases one's feeling of superiority and subsequently, one's self-esteem. Upwards social comparison, in the best case, motivates one to thrive for a more privileged place in society. In the worst case, it 
evokes greed and envy for what others have and thereby instils a feeling of inadequacy, frustration and unfairness in the individual. In a neoliberal society, everybody has thus become judge, jury and executioner of one's fellow human beings since this is one of the only means to establish a sense of self and essentially defines one's place in society (Hall, 2000, 2015).

This is, however, only one side of the coin. At the same time, how one is viewed by others has an impact on one's self-perception, which cannot be overestimated (Baars, 2013; Lyons, 2009; van Dyk, 2014). Most people in western societies consistently seek the approval, recognition and appreciation of other human beings since only through the positive feedback received, one can feel significant and can develop a positive self-concept. A lack of social recognition or social disapproval can potentially have detrimental consequences for the individual. Only through social feedback is the individual able to confirm what he or she perceives him- or herself to be and what one according to socio-cultural standards hegemonic at a particular time at a particular place ought to be. It could, I believe, even be argued that it is almost exclusively the reflection of oneself provided by others which enables the construction of a self in the first place and without it, one becomes invisible and somewhat ceases to exist (Bourdieu, 2001, 2018; Žižek, 2006, 2008, 2010).

In my view, the most crucial prerequisite for achieving positive social recognition in neoliberal societies is to prove to oneself and others that one is in control of one's life and this aspect must thus be viewed as representing a core element of individual identity constructions. Within this context, the development of life-course narratives is the precondition for the creation of coherence and meaning in one's life (Murray, 2003). They support integrating past experience with present actions, behaviours and aims and they enable envisioning one's future self. As such they are not objective accounts of our lives but purposive constructions that form a unified identity out of the contradictions every individual feels and is characterised by (McAdams, 2015; McAdams \& McLean, 2013). Life-course narratives, however, can, as discussed above, only be constructed within the narrow constraints of neoliberal master narratives and gain meaningfulness predominantly by sharing them with others (McAdams, 2015; McAdams \& McLean, 2013)

It is important to stress that cultural narratives understood in this way play a fundamental and indispensable role in the creation and interpretation of life as meaningful. They are carriers of values, they provide the horizon against which we situate our own life narratives. They provide us with a reservoir of both narrative form and content on which to model our own stories. (Laceulle \& Baars, 2014, p. 36)

Life-course narratives must, therefore, be seen as a means to generate both the illusion that one is the agent of one's lives instead of one's life being controlled by social reality (Eckersley, 2006; Laceulle \& Baars, 2014; Murray, 2003) and as an individual adaptation of 
social norms and rules aimed at establishing one's 'normality' to others which enables one's social acceptance and recognition.

Since the neoliberal master narratives of consumption, productivity and autonomy impact how one views him or herself and other people, they exert a substantial influence on the societal understanding of ageing and older age and simultaneously on how older people view themselves and on the life-course narratives they construct in older age (Laceulle \& Baars, 2014; Nelson \& Lindemann, 2001; Nelson, 2005). In general, the natural decline in productivity and a decrease in autonomy of ageing individuals and the celebration of youth as the prime time of productivity, independence and consumption in the west has led to rather negative views of ageing and an increasing loss of societal appreciation when ageing (Gullette Morganroth, 2004; Gullette Morganroth, 2008; Gullette Morganroth, 2011). Modern postcapitalist societies are characterised by their obsessive focus on personal autonomy and responsibility, advancement, speed and never standing still. It is thus no surprise that slowness, limited physical and mental abilities and a general inability to 'keeping up' with the pace of modern society, characteristics commonly linked to ageing, are understood as an impediment which is the result of personal failure (Baars, 2015). Consequently, if older individuals do not prove their value to society by staying independent, by contributing to society through being productive in voluntary work or at least by taking care of grandchildren and continue in their search for self-actualisation through consumption, they are often victims of discrimination and societal exclusion (Cruikshank, 2013; Featherstone \& Wernick, 1995; Gilleard \& Higgs, 2000).

I believe, however, that the application of Slavoj Žižek's explanation of racial phantasies (Žižek, 1992, 1997, 2006, 2008) to ageing can serve to illustrate another less apparent reason for the rather fearful and often bleak socio-cultural perceptions of ageing. In my view, the uneasiness linked to ageing and older age is ultimately rooted in fear of anything which resists unequivocal symbolisation (Žižek, 1997, 2006). Ageing and its endpoint, death, cannot be defined conclusively. In other words, although there is a signifying chain related to 'age' and 'ageing' it is highly unstable and ambiguous and predominantly characterised by the quality of 'not' - not being young, not being active, not being productive, not being mentally and physically fit, not having a job, not being technically minded (Cruikshank, 2013; Katz \& Marshall, 2003; Weedon, 2004), to mention but a few. It, therefore, resists coherent symbolisation and as such, threatens the individuals' need for unity and control of the universe surrounding him or her (Žižek, 1989, 2006). This feeling of instability and incoherence is amplified and gains relevance by the fact, that everybody ages, provided one lives long enough, and therefore will be part of the signifying chain linked to ageing (Chivers, 2011). 
To resovlve the ambiguity of the signifying chain linked to older age, the ageing person can, on the one hand, be viewed as the ethnic other in racist fantasies (Myers, 2004; Žižek, 1997). Current public discourses indicating that older people demand and drain resources that are meant to benefit the younger generation or the public damnation of older people whose way of life does not fit to the master narratives of neoliberal society are exemplary for sociocultural mechanisms at work when justifying societal exclusion and discrimination. Intergenerational conflicts and ageism are the results of this view of ageing (Gullette Morganroth, 2004, 2011). On the other hand, if older people stay productive, independent and continue to be consumers in their search for self-fulfilment, the quality 'not' can in today's western societal structures be suspended and the symbolic ambiguity of ageing and older chronological age can be overwritten (Baars, 2013; Carroll \& Bartlett, 2015; Cruikshank, 2013; Featherstone, 1995; Gilleard \& Higgs, 2000; Gullette Morganroth, 2004; Katz \& Calasanti, 2014). Subsequently age and the ageing process can be subsumed and fit neatly into current dominant narratives and loses the fear one feels when confronted with something unknown and undefinable. In summary, if adhering to the socio-cultural standards of neoliberal society, ageing is today socially acceptable but if an older person does not fulfil these standards it is legitimate to exclude him or her from society since he or she has obviously not tried hard enough to overcome the signs and inhibitions of ageing.

To enable a better understanding of dominant master narratives of ageing and its influence on the self-perception of older individuals in post-capitalism, I will, in the next section, critically review the constituents of the master narratives of ageing. After having discussed and highlighted the detrimental influence these master narratives can have on the selfperception of the ageing person, I will outline an alternative approach to viewing ageing. This is intended to avoid, even if unintended, a reproduction of the master narratives discussed (Baars, 2013; van Dyk, 2014) while highlighting an approach to ageing (Moglen, 2008a, 2008b) which could facilitate a more differentiated picture of older age in general, and particularly of older male age.

\subsection{Neoliberal Master Narratives of Older Age}

Since the process of bodily decay begins more or less in our twenties, ageing is something which affects everybody (Gullette Morganroth, 2004; Segal, 2013). Despite this fact, older age and the process of ageing remains a topic whose discussion is characterised by an overt and unprecedented level of simplification and homogenisation (Twigg \& Martin, 2015). Why is that so? 
Current western societies are characterised by dualism and the attempt to order everything in binaries (Fay, 1996; Tulle, 2015). This makes our complicated life, the choices we have to make, and the evaluation of our own and others' socially acceptable actions and behaviour much easier. Apart from the essential dichotomy of male versus female (compare chapter 4.1) (Bourdieu, 2017), another binary, the division between young and old, has been gaining increasing relevance since industrialisation (Addison, 2006) and developed into one of the master narratives of neoliberal society. It has, thus, become one of the crucial bases underlying individual identity formation when comparing oneself to others (Moglen, 2008a; Woodward, 1988).

The youth cult and with it its flip side, the pathologizing of ageing, characterising postmodern society, was firmly inscribed into our existence latest with the establishment of gerontology and geriatrics in the early $20^{\text {th }}$ century (Addison, 2006). Since then, ageing has become something which is perceived as an illness but also as something which can be managed and improved by medicine or medical procedures. "Ageing became something pathological that could be avoided - or at least delayed - if the proper preventive treatment(s) could be identified and undertaken," (Addison, 2010, p. 12). Resultantly, since the $20^{\text {th }}$ century the discussions surrounding ageing have centred on yet another binary construction: ageing as linked to decline and successful ageing or bad old age and good old age (Addison, 2010; Cruikshank, 2013; Featherstone, 1995; Gullette Morganroth, 2004; Katz \& Calasanti, 2014; Laceulle \& Baars, 2014; Moglen, 2008a; Segal, 2013; Woodward, 1988).

Lately, low fertility rates and advances in medical treatment and resulting prolonged life expectancy have led to a considerable increase in the older population in the west (Hoffmann et al., 2015; Kudo, Mutisya, \& Nagao, 2015). As a consequence of these demographic developments, the political and academic debates about ageing have gained momentum. As already highlighted above, the nightmarish visions produced by the media and politics of a greying population literally eating up resources, straining the social system and generally menacingly outnumbering the younger generation while demanding privileges which belong to the younger generation, characterises one side of the discourse surrounding the topic of older age (Calasanti \& King, 2015; Gullette Morganroth, 2008; Jennings, 2015; Katz, 2015). The negativity with which our society views 'bad old age' is not overly surprising given the fact that, as discussed above, neoliberalism is informed by the master narratives of autonomy and the related imperatives of agency and control, productivity and consumption (Baars, 2013). Precisely these attributes often subside with increasing age. It is a natural part of ageing that physical and mental abilities diminish, which often leads to lower-level productivity while the level of dependency on others might increase, and the consumption power often decreases. It, hence, resembles the horror scenario of the neoliberal individual: 
a lack of control over body and mind, dependency on others, limited financial means and a subsequent loss of status and privileges (Chivers, 2011; Cruikshank, 2013; Phoenix \& Griffin, 2015).

The negativity which with ageing is viewed is further amplified by the fact that even though people become much older than they used to, one is considered old at a much earlier time (Baars, 2015). Although in western industrialised countries one is generally considered old when approaching retirement at ages from 60 to 65 (WHO, 2011a), age becomes an issue, for women as much as for men, latest when we turn 40 (Cruikshank, 2013). Furthermore, western societies are characterised by their pathological fear of death. Since death not only represents the end of our existence but especially since it marks utter loss of control and a level of ambiguity and insecurity which is unprecedented in our lives, death and dying are one of the last taboos in western society (Calasanti \& King, 2015; Joyce, Loe, \& DiamondBrown, 2015; Tulle, 2015). It follows logically that the fear of ageing and death in combination with the media coverage and politico-economic debates of ageing have in western countries led to a rather negative view of ageing characterised by notions of dependency, frailty and decline (Gullette Morganroth, 2004; Wilson, 2000).

However, there is hope in sight, at least for the so-called young-old or people in their third age as compared to people who are old-old and in their fourth age. Alongside the cultural narrative of ageing viewed as decline today mostly linked to the 'fourth age' (Gilleard \& Higgs, 2000; Lloyd, 2015), an increasingly dominant alternative narrative has developed: the cultural narrative of successful ageing (Carroll \& Bartlett, 2015).

Already implied in the founding of gerontology and geriatrics, as highlighted above, in their book Successful aging: The MacArthur foundation study (1998), Rowe and Kahn formally defined how age can be managed successfully. This is possible only if people dedicate time to staying fit and refrain from unhealthy practices such as smoking, eating fast food or drinking to support mental and physical health into old age. In addition, Rowe and Kahn $(1997,1998)$ view the accumulation of financial resources enabling independence from state and family support, establishing sound and lasting interpersonal relationships and, regardless if retired or not, using one's time productively and in the service of society as key factors in enabling successful ageing:

To succeed ... means having desired it, planned it, worked for it. All these factors are critical to our view of aging which ... we regard as largely under the control of the individual. In short, successful aging is dependent upon individual choices and behaviors. It can be attained through individual choice and effort (Rowe \& Kahn, 1998, p. 37). 
Rowe and Kahn's understanding of successful ageing fundamentally rests on the cornerstones of neoliberal perspectives: individual autonomy, consumption and productivity (Carroll \& Bartlett, 2015). Emphasising individual agency releases the state from any responsibility for their older members. Highlighting lifestyle choices as imperative in increasing the chances of living a good old life places the burden of responsibility for life quality in older age in the hands of the individual. Simultaneously, it implies that age can be managed and that a positive ageing experience can be bought by being a good consumer who chooses the suitable consumer goods, may it be food or the appropriate and usually costly medical services. Lastly, indicating that effort and motivation are a prerequisite for successful ageing stresses that only if actively and productively approached, is it possible to be successful in controlling age and subsequently to age happily (Featherstone, 1995; Gilleard \& Higgs, 2000; Gullette Morganroth, 2004; Katz \& Calasanti, 2014; van Dyk, 2014).

The concept of successful ageing has been enthusiastically embraced by academia, politicians and media since it ties in well with the neoliberal agenda (Katz, 2015; Katz \& Calasanti, 2014). At the same time, on a subtler level, incorporating ageing into the value system of neoliberal society, allows its definition and evaluation and, thus, it loses its fearladen character while supporting the politico-economic system on two counts (Baars, 2013; Eckersley, 2006). Firstly, it firmly reminds the younger generation to plan well if intending to succeed in the process of ageing. Since this is majorly dependant on being successful and productive before retiring the active ageing paradigm facilitates the productivity dictate and the investment in consumer goods and services that promise a good life in older age. Secondly, the division of good and bad older age is, in its simplification and the resulting homogenisation and stereotyping of older people, an effective means to evaluate and to discriminate and exclude older people from society, if they do not comply with the criteria of successful ageing (Gullette Morganroth, 2004; Gullette Morganroth, 2008; Katz, 2015; Katz \& Calasanti, 2014). As such, successful ageing is a powerful tool in providing the illusion that age is controllable while anything which seems to prove the contrary is legitimately ignored, overlooked and/or condemned (Davey \& Glasgow, 2006; Gergen \& Gergen, 2000).

Consequently, western culture provides two frames - successful ageing and ageing as decline - for the construction of identity in later life (Calasanti \& King, 2015; Carroll \& Bartlett, 2015; Katz, 2015; Twigg \& Martin, 2015). As illustrated above, social structures and identity construction are inextricably linked (Bourdieu, 2017, 2018; Fay, 1987, 2014; Gullette Morganroth, 2004; Žižek, 1997, 2008). Thus, the question of what influence these master narratives of ageing have on ageing people arises. To decide between good old age 
and bad old age is, similarly to many other choices one seems to be making out of free will, however, no real choice (Bourdieu, 1990, 2017; Žižek, 1997, 2006). Indeed, who would willingly choose to be frail, dependent and mentally and/or physically limited? The choice is, contrary to public belief, predominantly not the individual's at all. The embodiment of age will have an impact on us regardless of how hard we try to fight, control or ignore it. As mentioned at the beginning of this chapter, the decline of bodily and mental abilities with increasing age is a natural process which cannot be reversed or stopped although it can be slowed down particularly if one is affluent enough (Chivers, 2011; Katz \& Calasanti, 2014; Katz \& Marshall, 2003; Tulle, 2015). Hence, age discrimination does not affect every older person in the same way. Ageism is therefore much less the result of chronological age but much more the outcome of class affiliation and, to a lesser degree, gender (Arber et al., 2003; Clover, 1993; Davenport, 2008; de Pater, Judge, \& Scott, 2014; Richardson, 2019; Woodward, 2006).

Anyone whose livelihood is determined by the investment of, in Bourdieu's words, physical capital is much more likely to not only be judged much harder but, particularly in the case of the blue-collar working class, also to age prematurely when compared to people who have a high level of cultural, economic and social capital at their disposal (Bourdieu, 1990, 2018; Tulle, 2015). The working class who usually have little cultural, social or economic capital, have not much else than their bodies and respectively their physicality to capitalise on. This can lead to an earlier bodily decline as bodies are overstretched and as the labour class usually do not have the money or, for a lack of knowledge or as a strategy of resistance, the motivation to compensate the abuse of their bodies by living healthily and/or access services facilitating a youthful appearance (Gilleard \& Higgs, 2000; Katz \& Calasanti, 2014; Swinnen \& Stotesbury, 2012). As a result, the process of ageing impacts working-class women and men in a double sense: they cannot capitalise on their bodies to earn a living anymore while being condemned by society for not having lived reasonably to avoid being a burden to society in older age.

Within this context, there can also be no doubt that in the social evaluation and classification of women, appearance still plays a major role. Female attractiveness and beauty are often the prerequisites for and the main focus of social recognition, prestige, status and, last but not least, employment (Chivers, 2011; Richardson, 2019). Resultantly women feel the pressures of ageing more than men do even though, in my view, there are other aspects linked to the ageing process which possibly hit men harder than women (see chapter 4) (Calasanti \& King, 2005, 2015). This is particularly true for women who are public figures such as actresses, politicians, singers, to name but a few. Nevertheless, these women usually also have the financial means to follow a healthy lifestyle, to afford costly fitness trainers and 
medical procedures which support the maintenance of a youthful look into older age (Clarke \& Bennett, 2015; Dolan, 2018; Marshall \& Lipscomb, 2010). This is not to say that people belonging to higher social classes or men are never victims of age discrimination, but it certainly means that anyone who has limited cultural, social and economic capital at his or her disposal is affected by ageing more severely (Cruikshank, 2013; McAdams, 2015; Price \& Livsey, 2015).

Indeed, the homogenisation of older people on the basis of generations or cohorts affects all older people even if in different degrees. In a society essentially based on continuous social, political and economic evaluation informed by numerical data and statistics and within binary frames (Jennings, 2015; Jennings \& Krainitzki, 2015; Tulle, 2015) such as effective versus ineffective, successful versus unsuccessful, productive versus unproductive, young versus old, to mention but a few, in combination with the emphasis on individual responsibility (Laceulle \& Baars, 2014), ageing, like everything else, has become a competition.

It is quite common that we often feel the pressure to fulfill the prerequisites of successful ageing from our mid-forties, if not younger. As a result, every breach of the more and more powerful dictatorial health and fitness trend increasingly linked to moral goodness and a lack of taking responsibility for securing the financial means for retirement causes an overwhelmingly bad conscience (Clarke \& Bennett, 2015; Gullette Morganroth, 2004, 2011; Hogan, 2016). Nobody wants to be a loser in the ageing competition and resultantly sacrifice societal privileges, respect, and above all, control of one's own life (Featherstone, 1995; Featherstone \& Wernick, 1995; Gullette Morganroth, 2003).

It is, therefore, no surprise that older people when asked about ageing usually compare themselves with other older people who do not fulfil the criteria of productive ageing while also frequently uttering that 'they don't feel old' or 'as old as their chronological age would suggest' (Nilsson et al., 2013; Thompson, Itzin, \& Abendstern, 1990; Tulle, 2015). This social comparison downwards enables the emphasis of the own success in ageing and therefore supports a positive self-perception in older age. Simultaneously, stressing that one's chronological age does not represent how one feels indicates, on the one hand, the internalisation of cultural master narratives of ageing linking older age to decline and, on the other hand, the inadequacy of chronometric time in capturing the self-perception of older people (compare next paragraphs).

All in all, there can be, in my view, no doubt that the active ageing paradigm puts enormous pressure on every older person who tries to comply with the standards set for good old age while it majorly contributes to the marginalisation and discrimination of those who cannot or - rarely - refuse to conform to the dictate of successful ageing (Arber et al., 2003; Clarke 
\& Bennett, 2015; Cruikshank, 2013; de Medeiros, 2016; van Dyk, 2014). I believe that ageing, like all human experiences, cannot sufficiently be aligned to binary constructions since life and subjective experiences are much more complicated than that (Baars, 2013, 2015; Moglen, 2008a; van Dyk, 2014). My view of the two cultural templates available for an ageing person is therefore rather critical. However, how is it possible to break out of the cultural matrixes of ageing provided by society so as not to, even if unwillingly, reproduce them when analysing the self-perception of older people (van Dyk, 2014)?

One main cornerstone of neoliberal society and, thus, the binary construction of ageing is chronometric time (Baars, 2015; Segal, 2013, 2014). The lives of anyone living in western societies are ordered and determined by watches, calendars, timetables and, most importantly, by our age. As such chronological time majorly contributes to the categorisation into generations or age cohorts and the related conceptions of what is considered to be appropriate at what age (Baars, 2013, 2015). The consequent homogenisation and simplification affect not only older people but everybody. In the desperate search for control, social recognition and a stable and consistent identity, individuals continuously try to conform to the age norms telling them what is viewed as standard and 'normal' at a particular life phase and where and what one should be at any particular age stage (Grenier, 2012; Gullette Morganroth, 2004).

Nonetheless, individuals do not experience time as orderly, objective measurement and chronometric. Instead, depending on its intensity and life stages, time runs slower or faster but never orderly and invariantly. As children, summer seems endless while the older one gets the faster time seems to pass, and one year is over in a flash. This aspect is particularly relevant with regards to the life-course narratives of older people (Baars, 2015). The older one gets, the more the past becomes intertwined with the present. The ambivalence most people feel when they see their ageing body and face reflected in the mirror and in the reaction of others and the age one feels to be are rarely synchronous, and less so the older one gets. That the visible and palpable signifiers of age strike people living in western cultures with such awe and horror is, on the one hand, undoubtedly the result of the culture we live in and its celebration of youth and productivity (Gilleard \& Higgs, 2000; Gullette Morganroth, 2008; Segal, 2008, 2013). On the other hand, the uncontrollability of ageing, which inhabits and occupies our bodies and minds against our will, is horrifying and leaves us feeling disoriented and somewhat helpless. It is, therefore, not surprising when one moans his or her lost youth and regrets missed chances and experiences (Moglen, 2008a; Segal, 2013).

'Transageing' or 'thinking with age rather than about age' might offer an alternative to the dichotomous, simplified, categorical and inherently destructive cultural understandings of 
ageing (Jennings, 2015; Jennings \& Krainitzki, 2015, unpublished; Moglen, 2008a, 2008b). If individuals manage to move away from chronometric and binary perceptions of their own ageing as well as in the attempts of understanding the experience of others with ageing and embrace a view of ageing as "the interrelated consciousness of many selves, which are of many ages and of no age at all," (Moglen, 2008a, p. 308) it might support the construction of a stable identity through positive life-course narratives across the entire life.

\section{The Construction of Men and Masculinities in Neoliberal Societies}

\subsection{The Construction of Hegemonic Masculinities in the West or Why do Men Always Have to Be in Control?}

Connell's now widely accepted definition of masculinity is a highly relational concept which reflects the ambiguity, temporality and the context-dependence of constructions of maleness throughout history and across the life span. As a cultural construct, masculinity is defined as the result of an interplay between the respective culture, the individual personality as expressed in actions and of positioning oneself in opposition to other men and women. “"Masculinity [...] is simultaneously a place in gender relations, the practice through which men and women engage that place in gender, and the effects of these practices in bodily experience, personality and culture,"(Connell, 2005, p. 71).

Understandings of masculinity thus depend and are affected by the geographical region, by the historical period and its respective values and norms as well as by societal and politicoeconomic developments. As such, for instance, changes in the status and evaluation of minority groups or changes in the work environment such as the increasing industrialisation or the transitions from an industrialised society to a service-oriented model or, more recently, the move towards an information-based mode of work, impact socio-cultural views of masculinity considerably (Cheng, 1999; Connell, 1993; Connell, 2005).

As well as these historical influences or these horizontal variables, several models of masculinity exist simultaneously at any moment in time within a specific cultural setting in the vertical axis of society. One of these masculinity ideals is usually dominant while other expressions of masculinity are less influential although they can and often do affect and modify the prevailing view of masculinity (Connell, 1993; Connell, 2005; Connell \& Messerschmidt, 2005). Connell has, apart from hegemonic masculinity, identified three additional masculinity constructions present at any particular moment of time in a specific society. Complicit men often live a life which in many aspects such as status, physical appearance, sexual orientation or lifestyle, do not comply with the hegemonic understanding 
of masculinity. Predominantly, however, they do not challenge the socio-cultural view of being a man but rather admire men fulfilling these standards. Subordination, on the other hand, characterises the status of men who cannot and/or do not want to accept the current view of masculinity such as homosexuals or effeminate men but clearly also the new breed of housemen. The 'wrong' race, low economic status and a low educational level determine the marginalisation of men (Connell, 2005). These constructions of masculinity are as context-bound as hegemonic views of masculinity, and as such, they are not only affected by historical developments but, throughout their lives, men possibly embody and enact different, hegemonic and less hegemonic narratives of masculinity (Cheng, 1999; Connell \& Messerschmidt, 2005; Edwards, 2004; Griffin, 2005).

However, as context-dependent as constructions of masculinity are, what seems to recur and tie together hegemonic understandings of masculinity across time is the significance attributed to being in control and to displaying and incessantly proving dominance. I believe this to be the result of the highly influential and seemingly unassailable binary construction of sexual difference. The unremitting socio-cultural power of the sex binary is astonishing when considering that the biological evidence for a division of humans into only two sexes is far from being unambiguous (Feinberg, 1996; Kessler, 1998; Rubin, 1997). In fact, there is plenty of proof of the existence of more than two sexes. This supports the view that, despite its claim for objectivity, the results of scientific research into the determination of sex were and are significantly influenced and interpreted in the light of the hegemonic socio-cultural understanding of the supposedly natural male-female divide. Its establishment as a scientific factum must, therefore, be seen as an essential constituent in preventing any changes in a system which ensures the privileged status of men while disadvantaging women (FaustoSterling, 2000; Lorber, 1993).

Nevertheless, the concept that our genitals, chromosomes and hormones do not clearly and unambiguously determine if we are men or women and albeit removing the category of man /woman altogether seems to pull the rug out right under our feet and leaves us clueless and disoriented (this is, at least, how felt when I first came across this approach). After all, what am I if I am not a man or a woman? Sexual difference is, without a doubt an, if not the essential base for our identity construction (Butler, 2011). In fact, sexual norms are so powerful that aspects which do not neatly fit into the socio-cultural gender norms of being either a man or a woman and related heterosexuality seriously threaten the unity of our selfperception. Deviations from the 'normal' are therefore often either suppressed or felt as detrimental inadequacy frequently leading to insecurity and potentially although less frequent, psychological trauma (Butler, 2011; Fausto-Sterling, 2008; Lorber, 1996). 
This is little surprising when considering that virtually every aspect of our lives can be broken down to connotations tied to language which are linked to the female/male divide. (Bourdieu, 2001). Indeed, social life is ineligible and manageable only since we appraise our behaviour, our actions, our identity, but also the animate and inanimate environment around us with reference to the binary sexual difference (Wittig, 1980, 1993).

The division between the sexes appears to be 'in the order of things', as people sometimes say to refer to what is normal, natural, to the point of being inevitable: it is present both -in the objectified state -in things (in the house, for example, every part of which is 'sexed'), in the whole social world, and in the embodied state in the habitus of the agents, functioning as systems of schemes of perception, thought and action. (Bourdieu, 2001, p. 8)

As a consequence, it is practically impossible for man as well as for women to avoid the socio-cultural and largely unconscious process of sexual indoctrination (Bourdieu, 2001) within which masculinity is associated with predominantly positively connoted aspects such as strength, dominance, rationality and control while connotations affiliated to femininity centre around notions of passivity, submission, helplessness, emotionality and empathy characteristics which are, in the occidental world, often valued considerably less than the supposedly male attributes. By linking male dominance and female submission to biological predetermination, patriarchy is defined as representing the natural order of things which legitimates its rule beyond doubt (Butler, 2011; Connell, 1998, 2005; Schippers, 2007).

The binary construction of sexual difference is so deeply implanted in the individual that one subconsciously embodies, enacts and confirms his or her gendered self in every role one plays on the stage of social interaction. "...the action of gender requires a performance that is repeated. This repetition is at once a reenactment [sic!] and reexperiencing of a set of meanings already socially established; and it is the mundane and ritualized form of their legitimation," (Butler, 2011, p. 191) Subconsciously gendering oneself univocally as a man or a woman is therefore essentially the foundation from which one cultivates all other aspects of his or her identity development. The “... objective complicity between the structures embodied in both women and men and the structures of the major institutions through which not only the masculine order but the whole social order is enacted and reproduced..."(Bourdieu, 2001, p. 117) renders challenging the identity constituting factor of sexual difference as nearly unthinkable.

If gender and sex are predominantly social constructions which reciprocally inform each other and if biological markers such as genitals, chromosomes and hormones cannot serve as unambiguous criteria for being a man, how can masculinity be defined and what exactly then makes a man a man? If maleness is predominately a construction and not something which is, at least partly, a natural embodiment than anything which is not in accordance with 
the respective socio-cultural understanding of true manliness is a threat which true men have to stand up to and put in a place of subordination. It is therefore crucial for men to establish their masculinity in opposition to constructions of femininity(Schippers, 2007).

From earliest childhood, boys are harshly made aware of what is considered to be unmanly demeanour such as playing with dolls, dressing too colourfully and flamboyantly, being emotional except in sports tournaments or perhaps when your child is born, showing weakness or admitting mistakes, and, most importantly, under no circumstances losing autonomy and agency. Any supposed feminine connoted conduct is immediately penalised in primary and secondary social groups by ridiculing the inability to be manly while media products, socio-cultural institutions as well as politico-economic structures enforce sociocultural understandings of appropriate masculine and feminine performance (Bourdieu, 2001; Butler, 2011; Fausto-Sterling, 2008). Breaching the socio-cultural standards linked to hegemonic constructions of masculinity will lead to immediate exclusion from male privilege (Cheng, 1999; Connell, 2005; Kimmel, 2001; Pyke, 1996).

Men thus feel the need to continuously reinstate and confirm their status since they are always in danger of losing their privileges to other men (and nowadays also women) and more importantly, their identity. In other words, to be masculine means to be in unremitting control, first and foremost, of the own male body and mind to prevent being taken hostage by the femininity surrounding men in-and outside of their materiality (Fausto-Sterling, 2000, 2008). This could explain the almost manic efforts of men who understand themselves as representatives of the current hegemonic masculinity to incessantly prove their dominance and superiority in any way possible (including violence) (Hall, 2002; Schippers, 2007).

Within this context, competition is at an early stage established as a practice whose outcome reflects the manliness of boys and men, which essentially determines their rank in the male hierarchy. As such sports and military service as well as career development and linked social and economic status are crucial elements of manhood and its constant need for displaying control and dominance (Baur \& Luedtke, 2008; Bourdieu, 2001; Coston \& Kimmel, 2012). "Male privilege is also a trap, and it has its negative side in the permanent tension and contention, sometimes verging on the absurd, imposed on every man by the duty to assert his manliness in all circumstances"(Bourdieu, 2001, p. 50)

Concepts of masculinity in the west are, on the one hand, thoroughly affected by the respective socio-cultural environment, whilst on the other hand, as I have argued, they are bound together across occidental cultures and historical periods by being based on notions of control and dominance. The next section is thus dedicated to analysing the significance of control in hegemonic constructions of German masculinities between 1935 and 1945 the years my interviewees were born in - without neglecting the specific socio-cultural 
situation Germany during these periods of time. This is aimed at gaining a better understanding of the masculinity constructs my interviewees were exposed to during their lifetime since, in my view, it can be assumed that these masculinity constructions exerted a significant influence on the way my interviewees view and enact their own masculinity.

\subsection{Hegemonic Constructions of German Masculinities}

Although understandings of masculinity in Germany overall mirror the general historical developments of masculinity in western industrialized countries, there are some aspects which seem to be the outcome of the specificities of German history (Baur \& Luedtke, 2008). Germany's low tolerance for uncertainty in combination with the high significance attributed to long-term planning enabling a stable future, the intense focus on individual performance and achievements particularly in a professional context and an emphasis on protestant work ethics, are, without a doubt, consequences of Germany's past (Hofstede, 1983, 2011). Men born between 1935 and 1945 were the sons of men who grew up in a time in which men felt they knew their place in a pecking order perceived as natural since it was believed to be legitimised by God and God's representative: the monarch. Hegemonic masculinity in the early $19^{\text {th }}$ century in Germany was, thus, predominantly represented by aristocratic men who were relatively immune against claims for dominance by other men although other masculinity constructs, such as the emotional and sensitive hero in the romantic literature of the time, existed (Colvin \& Davies, 2008; Gardiner, 2004).

The military, particularly in Prussia, had always been held in high esteem in Germany and especially its officers were seen as godlike but after the Prussian army defeated Napoleon in 1813 its socio-political power and influence markedly increased. It was in this time that attributes, such as rationality, discipline, authority, determination, mental and physical strength and a lack or, at least, a strict control of emotions, were firmly set as the characteristics representing true German masculinity - all virtues inextricably linked to the military and particularly to the Prussian army (Colvin \& Davies, 2008; Gray, 2000; Mosse, 1998).

The increasing industrialisation between 1815 and 1915 in Germany (Kiesewetter, 1996) slowly led to adaptations in the understanding of hegemonic masculinity. For capitalism to thrive, internal stability was needed since the production of goods required a harmonious and numerous workforce and citizens who were willing and able to consume goods without being distracted by internal conflicts and violence (Hall, 2002). "Social advancement became less dependent upon one's ability to wield arms and more dependent upon one's ability to compete with words and planned strategies with which to win the favour of social 
superiors."(Fletcher, 1997, p. 35) As a result, alongside the soldierly ideal of masculinity, the bourgeois and the ideal of the 'Bildungsbürger' (Gray, 2000; Hall, 2002; Willems, 1986) - a man who succeeded in climbing the social ladder by accumulating cultural and financial capital (Tjeder, 2003) and a predecessor of what Connell will later call "transnational business masculinity" (Connell, 1998, p. 16) - increasingly determined the standards of hegemonic constructions of masculinity. This masculinity concept was not exclusive, as it hardly ever is. It competed with the former still highly en vogue soldierly ideal and a newly developing working-class masculinity characterised by aspects such as muscularity, strength but also a lack of emotional control particularly with regards to anger, little cultural and economic capital and a general archaic savageness (Hall, 2000, 2002; Tjeder, 2003).

The masculinity constructs of 'Bildungsbürger', soldier, specifically officers, and workingclass men in Germany in the period between 1860 and 1918 were all characterised by the strict separation of the public and the private sphere (Gardiner, 2004). By establishing that women were naturally suited to staying at home and taking care of all private issues while men's natural role was to provide for the family and to direct and control the fate of the outside world, may it be in politics, business or war, to ensure the safety of the home, patriarchy was cemented as the primary defining principle of society (Gray, 2000; Kessel, 2003).

In the aftermath of World War I military defeat, political and economic instability during the establishment of the Weimar Republic and the transition from monarchy to nation-state and democracy, led to changes in hegemonic masculinity constructions. The increasing visibility and acceptance of women and homosexuals in liberal circles as a consequence of a socialist government and the rising influence of democratic socio-cultural orientation threatened the formerly axiomatic breadwinner model of hegemonic masculinity (McCormick, 2001). However, there was a strong oppositional movement at odds with the new and more liberal and modern understanding of gender roles (Fitterer, 2013).

In the numerous extremely conservatively oriented circles, democracy was viewed as a danger to true masculinity. Here the soldierly masculinity ideal of discipline, mental and physical strengths and agency and cognitive control was upheld and promoted in sports groups, the Freikorps (a paramilitary youth organisation), the boy scouts and, of course, the army in which liberalism and a softened idea of masculinity was established as the new enemy (Borutta \& Verheyen, 2010; Martschukat \& Stieglitz, 2018; Mosse, 1998).

When the National Socialists (Nazis) were elected as the strongest political party in 1933, it marked the end of liberalism and beginning of the distorted view of the German male 'Übermensch' (Ettelson, 2002). Nazi ideology promoted a masculinity concept based majorly on reinstating the old soldierly ideal promoted in above-mentioned ultraconservative 
circles which essentially fostered a nation ready for war (Kühne, 2009, 2010; Steiner, 2013). Hitler's megalomania and delusional attempts to establishing global dominance resulted in the Second World War, which brought unimaginable misery to the world. Subsequently and luckily, Germany was defeated. The German soldiers who came back from war returned to an occupied and majorly destroyed Germany in which women had started rebuilding the country and children had often no memory of their fathers, let alone a relationship to them (Borutta \& Verheyen, 2010). They also had to face the shame of having, knowingly or not, supported a regime which provoked World War II and which had, with German efficiency, murdered six million Jews not to mention the thousands of homosexuals, non-German nationals and partisans (Bauman, 2000).

Although there was a short period of time directly after WWII characterised by efforts of denazification, the remaining men, as well as the whole nation, were quickly given a general pardon. This was a strategic move since the geographical location of Germany was seen as crucial by western allied powers. It, therefore, needed to become stabilised and strengthened so that it could serve as a bastion against the developing communist east bloc (Gardiner, 2004; Maier, 1977; Taylor, 2011). Although understandable, this strategy also led to former Nazis being reinstated in crucial positions in the legal systems as judges, in educational institutions as teachers and professors and in the political system as ministers, to mention but a few. At the same time, the general insecurity felt after WWII, and the newly developing threat of the cold war and communism resulted in a wide-reaching new conservatism in the west. Subsequently, developments towards a more modern understanding of masculinity and femininity faltered in the face of the heavily promoted picture of the family unit consisting of a mother, a father and one to two children as an essential foundation for moral stability and economic prosperity (Lazar \& Mauch, 2004).

My interviewees were thus socialised in an environment in which their mothers were frequently the primary caregiver because their fathers had either not come back from the front or if they did, were struggling mentally and physically with the aftermath of the holocaust, WWII and their share in it and, as a result, in fulfilling their role as breadwinner and patriarch (Jeffords, 1998; Moeller, 1998). To compensate their feeling of lost agency and failure, these fathers were often extraordinarily authoritative and emotionally cold since that was what they had learned to be the suitable masculine role in the family (Poiger, 1998; van Rahden, 2010). In school and university, many of the generation born between 1935 and 1945 were taught by former Nazis who promoted ultraconservative values and standards such as heterosexuality, fulfilling one's role as breadwinner or respectively housewife and mother, and being disciplined and obedient to authority. Simultaneously, the youth in the 50 s and early 60s was more and more influenced by the occupier's and particularly the 
American culture. Its media products and consumer goods perpetuated narratives of the nuclear family as the palladium of normality and principally also of social and economic success whilst stressing the significance of individual agency and stamina for societal ascent (Lazar \& Mauch, 2004) .

When the younger generation realised the extent of their parents' and generally the entire older generation's part in the horrors of Nazi regime, the holocaust and in WWII, it led to an overwhelming feeling of disappointment and distrust in older adults which stirred up an attitude of resistance. This found its expression in the student revolt of the late $60 \mathrm{~s}$. As in other parts of the world, the student revolt and other civil rights movements resulted, on the one hand, in the development of Hippie communities fostering tolerance, equal rights and sexual freedom and, on the other hand, in the emergence of extremist left-wing organisations such as the Red Army Faction (RAF) which terrorised Germany in the 70s. Although the 70 s were, thus, a time of socio-cultural upheaval and insecurity, they also paved the way for a more flexible and tolerant understanding of gender relations (Brown, 2013). Conservative views of what it meant to be a man were questioned and replaced by alternative understandings of masculinity. Suddenly men were expected to show emotions, be loving to their children, take part in their upbringing, take on household tasks and respect women as their equals (Gotto, 2017).

However, the breadwinner narrative was still maintained and extremely persistent in many influential and more conservative-oriented social institutions such as the church, right-wing governments, such as the 16-year reign of the CDU, or the army in which all men had to serve a basic military service of 12 to 18 months right after school (Gardiner, 2004). German men born between 1935 and 1945 were hence exposed to two masculinity constructs during their younger years - one rather conservative emphasising that men's primary role is to provide for their family and another more liberal one stressing the equality of men and women and demanding men to dispose of any conservative understanding of masculinity (Baur \& Luedtke, 2008).

To complicate German male identity constructions further, by the mid-eighties changes in the work environment and increasingly robust implementation of neoliberalism as the dominating politico-economic system resulted in a hegemonic understanding of masculinity "marked by increasing egocentrism, very conditional loyalties (even to the corporation) and a declining sense of responsibility for others (except for the purposes of image-making)..." (Connell, 1998, p. 16). Globalisation, growing market orientation and the immense power of multinational corporations in current western society has resulted in defining successful masculinity as mainly based on its access to power. As such, it must be viewed as a logical continuation of older models of manhood linked to control, agency and the subordination of 
others. At the same time, however, formerly crucial elements such as physical strength and domination, sexual orientation and related family structure as well as the understanding that part of being a man is to fight and protect one's fatherland and its values have become less crucial and replaced by a focus on individual agency and responsibility.

On the other hand, when East and West Germany were united in 1990 this not only ended the cold war between Eastern and Western powers and related worldviews based on socialism and capitalism respectively which lasted 40 years, but it also extended the constructions of masculinity that were available. This was especially so in terms of the ways that West German men became aware of East German understandings of masculinity that were strongly informed by socialist values aimed at enforcing equality in all areas of social life. However, the decline and perceived failure of socialist ideology, not only in Germany but all over the world, has frequently been used in a re-unified Germany to devalue and marginalise the socio-cultural values and standards linked to socialism. This is particularly the case if it is acknowledged that, when compared to West Germany, East Germany was much more successful in attempting to remove gender hierarchies and their linked conceptions of masculinity and femininity (Brandes, 2007). As such, East German understandings of masculinity must be viewed as representing a marginalised concept of masculinity in Germany and it can be assumed that it did not affect the identity construction of my interviewees especially since none of them was exposed to former Eastern German constructions of masculinity in their private or professional contexts.

German men who are now between 70 and 80 years old were thus affected by three hegemonic masculinity models during their life-course. Early in their upbringing, they were, mostly through their fathers or other older men in their social surroundings, exposed to the breadwinner model defined by ultra-conservative values such as a clear separation between public and private sphere, normative heterosexuality and explicit patriarchy. During their university and/or early employment period, the alternative masculinity model of the 'softie' developed. It questioned the old-fashioned view of the breadwinner construct and demanded of men to allow their feminine side to come to light, do away with all tendencies of patriarchal conduct and to live a life in which men and women are in all areas, public and private, equals (Colvin \& Davies, 2008; Gardiner, 2004). Alongside this masculinity concept, the transition from capitalism to the domination of the socio-cultural and politicoeconomic system of neoliberalism in the western hemisphere has produced an understanding of hegemonic masculinity mostly determined by power and control. Although inherently patriarchal, its focus on power allows for certain flexibilities in formerly viewed as natural constituents of masculinity such as heterosexuality or physical power, (Baur \& Luedtke, 2008). It is my argument that the interviews with my participants which follow in this thesis 
enable particularly exciting and revealing insights into the identity construction of older German men who grew up in a rather turbulent time characterised by varying and contradictory masculinity constructions and in a country in which regulations, norms, rules and uncertainty avoidance are held in the highest esteem.

\subsection{Controlling the Uncontrollable: Older Age \& Hegemonic Constructions of Masculinity in Neoliberal Societies}

In current meritocracies, outstanding achievements in one area might override a disadvantaged position in another constituent of hegemonic masculinity. For instance, homosexuality which usually disqualifies a man outright from hegemony could be superseded by exceptional performances in sports provided that one does not display his homosexuality too openly (Beynon, 2001; Schippers, 2007). The question at the core of this thesis is, however, does this also hold true for older men? Can men keep their hegemonic status also in older age, do they even aspire to remain representatives of hegemonic masculinity and, if so, what are the prerequisites for hegemonic male status in older age?

It is astonishing that although several studies in different countries have researched and documented varieties of masculinities throughout history and region (Law, Campbell, \& Dolan, 1999; Roberson \& Suzuki, 2005), strikingly little attention has been paid to older men or to the fact that age might affect their status (Calasanti \& King, 2005; Jackson, 2016; Spector-Mersel, 2006). In other words, not only race, religious and sexual orientation, economic status or educational level might contribute to a complicit, subordinate or marginalised status of men but, given the public perception of age, also chronological age might play a crucial role in the perception of men by others and themselves (Calasanti \& King, 2005; Chivers, 2011).

That men are mostly absent from the discussions of ageing is even more notable given the fact that in recent years and as a result of the demographic change in the western world, research about ageing has increased immensely. Most of this research, however, has focussed either on generally highlighting the challenges and opportunities of ageing for a rather faceless and basically sexless anonymous mass of elders to instigate necessary changes in our view and treatment of seniors or reflected on the difficulties involved in female ageing (Arber et al., 2003; de Medeiros, 2005; Milner, Van Norman, \& Milner, 2012; Moglen, 2008a; Segal, 2013; Tulle, 2015; van Dyk, 2014).

As discussed in chapter 3.2 of this thesis, undoubtedly, women are particularly disadvantaged by the ageing process. Not only is their social status throughout their lifecourse still predominately underprivileged and majorly determined by their appearance and 
attractiveness at every stage of their lives but especially in older age but they also suffer more often and as a result of their underprivileged status from financial instability in older age which frequently leads to social exclusion and isolation (Hogan \& Warren, 2012; Segal, 2014). However and despite the fact that men in a patriarchal system are always and also as older men privileged, I believe that the process of ageing affects men's self-perception and social appraisal as severely as women's (Jackson, 2016). The hegemonic cultural narratives of ageing contextualised in society as being successful or as decline lead to social marginalisation and segregation of women and men alike and, thus, the construction of ageing in neoliberal society is, in my view and contrary to some scholars (Addison, 2010; Cruikshank, 2013), not a gender-specific phenomenon although it undoubtedly affects men and women differently (Arber, Davidson, and Ginn 2003, Featherstone and Wernick 1995, Hearn 1995).

Even at the risk of oversimplifying, hegemonic masculinity in postmodern western society is majorly dependant on displaying control and dominance. Primary vehicles for this are, on the one hand, heterosexual potency and, on the other hand, employment which is the basis for the accruement of status symbols such as a house, a car, expensive holidays, to mention but a few, which then, in turn, determine the position men can claim within the framework of the hegemonic masculinity constructions available today (Calasanti \& King, 2005; Connell \& Messerschmidt, 2005; Griffin, 2005; Hearn, 2004; Hearn \& Sandberg, 2009; Mann, Tarrant, \& Leeson, 2016). Precisely these aspects are affected by the process of ageing.

Thus, the question arises as to how men who have retired and who most likely experience a decrease in capabilities including in sexual potency, handle these age-related changes and how they incorporate aspects linked to ageing into the construction of an older male identity. Non-hegemonic status demands of the individual to find ways of maintaining self-worth and identity despite being marginalised (Calasanti \& King, 2015; Coston \& Kimmel, 2012; Tarrant, 2010). But what do men, who have during major periods of their adult lives, been part of the hegemonic class of men and who were as a result never forced to find ways of handling marginalisation, do? How do they deal with the natural decline in physical and mental abilities and linked reduced level of control and the loss of the identity-constituting professional role in older age and with the ageism prevalent in western societies?

As highlighted above, research on older men in their third or fourth age is limited. Studies discussing male ageing often do so within the context of the media portrayal of older men in advertising and feature films or they are purely theoretical (Boyle \& Brayton, 2012; Calasanti \& King, 2005; Chivers, 2011; Collopy, 2015; Fung, 2013; Hearn \& Sandberg, 2009; Holmlund, 2010; Holt \& Thompson, 2004; Saxton \& Cole, 2013; Spector-Mersel, 
2006) while very few empirical investigation of older men's live-course narratives exist (Alex, Hammarström, Norberg, \& Lundman, 2008; Capstick \& Clegg, 2013; Jackson, 2016; Mann et al., 2016; Phoenix \& Griffin, 2015; Thompson, 1994). The available literature emphasises that the hegemonic cultural narratives of ageing in neoliberal western society are highly influential in the way older men see themselves as well as in the way they are portrayed in the mass media (Chivers, 2011; Jackson, 2016).

The narrative of decline as the necessary consequence of ageing plays a similarly significant role in the socio-cultural understanding of older male age as it does in older female age. While the focus with women often lays on the decline of their physical attractiveness and consequent social isolation, with men the decrease of potency, status and physical and mental control and autonomy is emphasised (Chivers, 2011; Jennings \& Krainitzki, unpublished; Jennings \& Oro-Piqueras, 2016; Moore \& Reynolds, 2016). Within this context, older men are often portrayed in mass media as suffering immensely by the lack of a useful role (Spector-Mersel, 2006) in older age as a result of being deprived of a paid job. For older men, it is, contrary to older women, apparently not fulfilling enough to be a husband, friend or grandfather (Chivers, 2011; Hearn, 1995).

Indeed, it is striking that in media products, older men are often shown as living in complete isolation. They are either divorced, widowed or have never married, they frequently have no or very loose family relations and a practically non-existent social circle except for the perhaps one close friend - a result, of course, of their obsessive focus on work (King, 2007; Tarrant, 2010). As a consequence of not being able to comply with crucial benchmarks of hegemonic masculinity, such as being in complete control of oneself as well as everybody around them and the lack of a meaningful role confirming this control, related status and importance, older men are stripped of many of the male privileges they are used to and are shown as having immense trouble in reconstructing their identity on the basis of the changed circumstances in older age (Jackson, 2016). Thus, older men are stereotypically portrayed as embittered, grumpy and resigned and, partly also as asexual or feminised beings. Or, on the contrary, represented as sometimes not being in control of their sexual desires and therefore becoming 'dirty old men' (Calasanti \& King, 2005).

Older men therefore face a rather complex situation when trying to adapt their life-course narratives to their ageing selves. Their identity construction as men remains closely linked to displaying control and dominance while the available socio-cultural frames of older age, which are based on the homogenisation of older people, emphasise an unavoidable loss of bodily and mental control and disability and related decline. This reduction in social value considerably lessens men's access to male privileges and socio-cultural power they have been used to and based their identity construction on. "The threatening loss of socio- 
economic productivity, social status, fears of sexual impotence and the disorientating shocks of physical disruptions and discontinuities ...often create a destabilising, social contexts where dominant ideals of masculinity and gendered expectations are weakened (Jackson, 2016, p. 54)." No wonder then that men of all ages are horrified by the process of ageing if this is the cultural repository of possible old age available to them.

However, as examined in chapter 3.2 of this thesis, in recent years, a compelling counternarrative to the socio-cultural understanding of ageing as decline has developed. Men no longer have to live in fear of an older age characterised by impotency, loss of control and autonomy and following dependence provided they age successfully. The concept of successful ageing is based on the understanding that ageing can be managed and controlled if one dutifully follows the neoliberal dictate of taking individual responsibility for ageing actively and productively or, in other words, for staying youthful as long as humanly possible (Featherstone, 1995; Katz \& Calasanti, 2014). It goes without saying that the chances for successful ageing are significantly influenced by the economic and social capital one has at his disposal (Bourdieu, 2018). In a nutshell: the less you have of both, the lower your chances of becoming a successfully ageing man are (Laceulle \& Baars, 2014).

This is, however, not part of the story being told in the media and perpetuated on the micro as well as macro levels of society. Instead, we are told a story in which men and women who age unsuccessfully can only blame themselves for not having tried hard enough (Hearn \& Sandberg, 2009). A narrative which finds its extension and apogee in the rather new narrative of a healthy lifestyle. This narrative is not only based on emphasising that fitness and health consciousness are the prerequisites for a happy and prosperous life at any life stage, but it is also characterised by the condemnation of everyone who does not follow this health dictate since apparently, it is an indication for a weak character and a lack of will power as well as of moral deficiencies (Hogan, 2016).

In neoliberal societies which primarily evaluate everything and everybody by the respective market value, men can and do retain some of their male privileges if they are good neoliberal citizens and do everything in their power to support everlasting youthfulness by individual determination and by consuming products and services which aid the prevention or at least deferral of age-related mental and bodily decay (Cornwall, Karioris, \& Lindisfarne, 2016; Griffin, 2005). Viagra has enabled men to stay sexually active (Moore \& Reynolds, 2016) regardless of age while being sportive and socially active throughout one's life is communicated in public discourse as ensuring physical and mental fitness in older age (Beynon, 2001; Kimmel, 2001). Subsequently, autonomy and control can be retained, which is also supported by taking part in recreational activities such as long and expensive holidays to exotic places (Tulle, 2015). 
However, there can be no doubt that "[E]ven when blessed with the privileges of money and whiteness, old men lack two of hegemonic masculinity's fundamentals: hard-charging careers and robust physical strength,'(Calasanti \& King, 2005, p. 10). Nonetheless, even if older men cannot compete with younger men - although they might try - they can continue striving for dominance and superiority over women - older or not - and other older men to ensure themselves and others that they are still powerful and therefore can demand the privileges reserved for the hegemonic class of men (Chivers, 2011). Male ageing has, therefore, become yet another competition in which ageing as decline is the enemy and successful ageing is the key constituent not only for a fruitful and bright third and fourth age but also for staying top dog, at least among the marginalised group of older men and women. Men who used to live a life predominantly embodying hegemonic masculinity can retain a feeling of agency, usefulness and potency as long as they are fitter, healthier, more affluent and, most importantly, more in control than particularly women, regardless of age, but also other older men (Connell, 1998; Hall, 2002; Kimmel, 2001).

Nevertheless, despite the fact that age-related physical and mental decline can, if you are fortunate enough - in the double sense of the word - be postponed, it cannot be stopped. Fighting older age is, thus, an uphill struggle doomed to fail. As traumatic as this realisation might be for many men, it might also open up the opportunity to critically reflect on formerly held beliefs and convictions of what constitutes being a real man and lead to alternative constructions of manhood in older age (Jackson, 2016).

The film analysis following this chapter aims to generate more insights into the public discourse linked to older age and constructions of masculinity since, in my view, U.S. mass media products and particularly films play a crucial role in the preservation and dissemination of dominant socio-cultural values. Within the context, the recent revival of tough-guy action films is especially interesting since the films revolve around aged workingclass men who are in stark contrast to the current hegemonic masculinity ideal of whitecollar, young to middle-aged, affluent, middle- and upper-class managers. The critical examination of the portrayal of men in The Expendables trilogy should, therefore, enable a better understanding of the cultural frames which are available to men to alleviate and neutralize narratives of disability, decline and stigma so as to enable the construction of acceptable, sustainable and positive identities in older age. Subsequently, the interviews with my participants were motivated and informed by the intention to identify how older (German) men use the socio-cultural frames highlighted in chapters two, three and four of this thesis to construct identity in their life-course narratives as older German men. 


\section{The Construction of Male Ageing in the Geriaction Film Trilogy The Expendables}

\subsection{The Expendables Trilogy}

The media, and particularly mass media products, are today main agents in the dissemination and perpetuation of master narratives shaping public as well as interpersonal discourses (Dolan, 2018). Films, TV programmes, magazines and advertising produced for the international market have in recent times increasingly been at the centre of scholarly attention since "they are much more influential than the narratives that are not backed up by societal power and concentrate less on narrative persuasion" (Baars, 2013, p. 10). As discussed above, Rowe and Kahn's (1998) concept of successful ageing developed in the 90s had a significant impact on the public discourses linked to ageing (Lyons, 2009; Segal, 2013). As had the increasing visibility of the demographic change and the rising significance of a neoliberal value system in the west. The transition from capitalism to neoliberalism, the emergence of the successful ageing paradigm and the palpable rise in older population groups, have in combination led to a shifting emphasis in the public representation of ageing in the past thirty years. All of a sudden, or so it seems when looking back, older people moved from an invisible status to the limelight and became part of the media landscape. They were not only targeted in advertisements promoting age-defying products and services but also older people were given centre stage in a number of films and series produced for the international mass market (Dolan, 2018).

Films such as the British production The Best Exotic Hotel Marigold (Madden, 2012), the Hollywood production Something's Gotta Give (Meyers, 2003) or the U.S. American series Grace and Frankie (Kauffman \& Morris, 2015, 2016, 2017, 2018) feature actresses and actors aged between 50 and 70 years and were substantial commercial and critical successes. This rising interest in the older generation is also evident in the many discussions in the press focusing either on how well celebrities have aged or on how older population groups have carelessly been neglected as a consumer group (Mandelbaum, 2013; Murtaugh, 2017; Patterson, 2014; Tucker, 2014). Older age is today transmitted in the media as something which can and must be managed successfully by the individual through meaningful contributions to society, through living healthily and autonomously and through consuming whatever products and services are necessary to keep age in check. This apparently enables, at least fictional characters and celebrities to stay if not forever young then at least much younger than one's chronological age would suggest for much longer than in previous decades thought possible (Chivers, 2011; Dolan, 2018). 
In fact, colloid images of older age in films, on TV, in magazines or in print or audio-visual advertising campaigns seem to be visions of agelessness and seem to be somehow frozen in time. As such current portrayals of ageing in the media are increasingly characterised by excluding everything which is abject and horrifying about ageing and by presenting a sugarcoated image of ageing that does not disturb the sensitive neoliberal individual by purporting the belief that one is the master of one's own destiny, at least until finally being robbed of control by death. In other words, despite the fact that "an increase in films about ageing indicates a greater social concern about old people, in fact, the films reflect an ongoing pathologization of changes associated with age" (Chivers, 2011, p. 147).

An essential element of all discussions of ageing discourses in the media is an examination of the link between ageing and gender. Within my research context, the emergence of the so-called geri-action film in the past 20 years is of particular interest (Calasanti \& King, 2015; Cruikshank, 2013; Holmlund, 2002; Jermyn \& Holmes, 2015). The beginning of the $21^{\text {st }}$ century saw a sudden upsurge in action films closely resembling the tough-guy action films of the 1980s in which not only the storyline was similar or in fact a continuation of the original narration but also the actors predominantly remained the same. In other words, these films are a revised edition of a tried and tested formulaic action narrative featuring recognisibly hard-bodied but aged action heroes and based on a rather archaic, macho masculinity that has little awareness of more politically correct or palatable attitudes to gender. Besides the age of the protagonists, on the surface, none of these geri-action films is a surprise at all (Tasker, 1993a). Hyper-muscular men with no, mysterious or problematic family ties, fight injustice from a disadvantaged position. They face a massively superior opponent and are armed only with male camaraderie, true loyalty, authentic values and most importantly, un-human physical strength and agility and, of course, they are, in the end, victorious (Boyle \& Brayton, 2012; Lennard, 2014).

In The Expendables Barney Ross (Sylvester Stallone) and his team of mercenaries (Jason Statham, Jet Li, Dolph Lundgren and Terry Crews) the 'Expendables' are offered 3 million dollars to kill the corrupt dictator General Garza who rules Vilena, an island in the Gulf of Mexico, by Mr Church (Bruce Willis). Barney and his partner Christmas travel to Vilena to meet their local contact, Sandra. Although Barney and Christmas manage to escape a fierce fight with the dictator's troops, Sandra refuses to leave her country and is left behind. After some investigation, Barney finds out that Church is an FBI agent who actually did not hire him and his team to save Vilena from a dictator but rather to do the dirty work of killing the powerful drug dealer and rogue FBI agent James Munroe, who uses the island to grow cocaine. Despite feeling betrayed by Mr Church, Barney and his team return to the island, 
kill Munroe and save Sandra who remains on the island to rebuild it and restore peace and democracy (Stallone, 2010).

In The Expendables 2, CIA agent Church (Bruce Willis) offers Barney Ross (Sylvester Stallone) the seemingly simple job of retrieving the contents from a safe in a crashed plane. As part of the agreement between Barney and Church, the team is joined by Maggie, a young woman who is a specialist in cracking security codes, among other things. Despite some difficulty, the team manage to rescue the box. However, while returning to the plane, they find that their sniper Billy The Kid (Liam Hemsworth), the youngest member of the team, has been taken hostage by a group of men under the command of Villain (Jean-Claude Van Damme). Although Barney exchanges the box for the life of Billy the Kid, Villain kills him before leaving in a helicopter. When Barney is told by Maggie that the content of the box shows the locations of a massive mine of Russian plutonium, Barney and his men decide to track down Villain and his group known as The Sangs and kill them. This, they then do, and on top save the world from a nuclear threat (West, 2012).

In The Expendables 3 Barney (Sylvester Stallone), Christmas (Jason Statham) and the rest of the team comes face-to-face with Conrad Stonebanks (Mel Gibson), who years ago was co-founder and fellow combatant of The Expendables. Stonebanks subsequently, however, motivated by greed turned bad instead of fighting for justice - something intolerable for Barney. Since Barney once unsuccessfully tried killing Stonebanks, he has now made it his mission to erase the Expendables. Confronted with this grave threat, Barney decides that he has to fight old blood with new blood, and brings in a new era of the Expendables team members who are younger, faster and more tech-savvy or so it seems. The latest mission, therefore, becomes a clash of classic old-school style versus high-tech expertise in which, in the end, and not really surprisingly, the old save the young from certain death (Hughes, 2014).

The main cast in The Expendables, The Expendables 2 and The Expendables 3 is between forty-four and seventy-two years old. The main character Barney Ross (Sylvester Stallone) is sixty-four in the first film, sixty-six in the second and sixty-eight in the last. The remaining crew is considerably younger. Mr Christmas (Jason Stratham), Hale Caesar (Terry Crews), Toll Road (Randy Couture) and Yin Yang (Jet Li) are all between mid- and late-forties, and Gunner Jensen (Dolph Lundgren) is in his mid-fifties. Supporting roles, including the opponents, are predominantly filled by older men of the likes of Mickey Rourke (58), Eric Roberts (54) or Bruce Willis (55) in The Expendables or Chuck Norris (72), Jean-Claude Van Damme (52) and Arnold Schwarzenegger (cast in all three films) (65) in The Expendables 2 and 3. 
As said elsewhere (Bühring, 2017), to cast older actors between forty and end sixties as action heroes is atypical for the genre of action films (Tasker, 2004). The chronological age of the majority of the cast is in stark contrast to the current hegemonic masculinity ideal of white-collar, young to middle-aged, affluent, middle- and upper-class managers (Coston \& Kimmel, 2012; Pyke, 1996). The high commercial success of the films in the U.S. and abroad (Mojo Box Office, 2010, 2012, 2014) suggests that the films present male characters which are, despite their chronological age (and working-class social status), embraced by audiences as socio-culturally acceptable representations of older age masculinity. Hence, the examination of representations of masculinity in older age in the selected films will allow for particularly revealing insights into a neoliberal society essentially characterised by patriarchal dominance and enable a better understanding of "the context within which contemporary older men struggle to build acceptable identities," (Spector-Mersel, 2006, p. 68). The analysis, hence, focuses on the strategies used in the Expendables trilogy to transform narratives of decline commonly linked to ageing into stories of success and progress with a strong market value.

\subsection{Narratives of Masculinity in The Expendables Trilogy}

As discussed previously, masculinity, and particularly hegemonic masculinity, is not easily earned and from earliest childhood on needs to be continuously proved and reinstated. Physical strengths and mental prowess as well as being efficient and in control of oneself are crucial prerequisites in the daily and life-long competition for male privileges and hegemonic status (Bourdieu, 2001; Connell \& Messerschmidt, 2005). Ageing poses a severe threat to these cornerstones of dominant masculinity. Earlier or later natural biological decline impacts bodily control, and mental velocity whilst retirement robs many men of a powerful means for gaining status and recognition, namely a meaningful and lucrative occupation (Dolan, 2018; Donnar, 2016; Lennard, 2014).

Yet, the portrayal of older masculinity in geri-action films presents older men as far from having lost any of the aspects ensuring male dominance. Signs of ageing are visible in, for instance, the wrinkled faces and hands or a reduced eye-sight, whilst other parts of the body are often covered in clothes in contrast to being on continuous display in earlier performances of the protagonists (Lehman, 2001; Lennard, 2014). That the male body in the action films of the $80 \mathrm{~s}$ was orchestrated as a sexualised object of desire for both the male and female audiences - something traditionally reserved for the male gaze on female protagonists (Mulvey, 1992 [1975]) - has been highlighted by a number of scholars (Feasey, 2011; Holmlund, 2002; Neale, 1983; Tasker, 1993a, 2004, 2012). It is therefore striking that 
in The Expendables trilogy as well as other geri-action films the older male (and female) bodies are mostly hidden by clothes or the focus on bodies is deflected by the action taking place around them. The stark contrast to the way their hypermuscular bodies were staged in the protagonists' earlier films indicates that the visibility of physical markers of age on the bodies of the older cast were seen as interfering with their construction as chronologically older men whose abilities and powers are yet untainted by their older age (Donnar, 2016). Indeed, the narrative construction of the films invests a considerable amount of time and effort to assure audiences that older members of The Expendables have not lost any of their abilities that are indicative for previous stages of their on- and off-screen lives. In fact, it seems that their abilities have rather increased than declined. Each of the three films begins with high-speed, destructive and action-packed scenes. The filmic exposition quickly and firmly establishes that the higher age of the protagonists has not in any way reduced the nearly superhuman capabilities of the members of the Expendables team. At first sight, it seems that the hyper-muscularity and extraordinary cleverness of the male and very occasionally female cast, the high-tech and over-the-top armament and the spectacular action scenes merely comply to genre-typical standards and that the older age of the cast is simply ignored (Dolan, 2018). However, in my view, disregarding the chronological age of the protagonists outright would have decreased their authenticity and believability and veered the films towards a slightly absurd comedy at risk of exposing the older cast to ridicule. Instead, their portrayal is based on a highly complex dramaturgy in which narratives of successful ageing and ageing as decline are skilfully interwoven with narratives of masculinity.

Although the films are clearly based on a rather conventional and easily foreseeable genretypical storyline (Tasker, 2012, 2015), it also tells a story of older men battling their decline by embodying a masculinity ideal which, despite the fact that it is apparently outdated and anachronistic, disproves their perceived 'expendability' for neoliberal society (Boyle \& Brayton, 2012). The Expendables trilogy transmits that it is precisely the roughness and toughness of this allegedly outdated understanding of masculinity which not only allows the aged action heroes to fight and conquer decline linked to ageing but also ultimately enables them to save the world. Notwithstanding this, the portrayal of the ageing action heroes in The Expendables trilogy is not without facets in the depiction of their main characters - an indication for the scriptwriters' awareness for the negative connotations related to narratives of ageing and its perceived impact on male virility (Cruikshank, 2013; Dolan, 2018).

As discussed above, male status is crucially linked to potency, employment and on displaying control of oneself and others (Donnar, 2016; Feasey, 2011; King, 2007). The Expendables trilogy wastes no time to establish the high relevance and meaningfulness of 
the job the Expendables team perform. The protagonists of the films are mercenaries. They are hired to do jobs that are dirty and highly dangerous but need to be done to save humanity - clearly an occupation whose significance surpasses the relevance of any other job imaginable. This is further underlined by depicting the team as having clear moral values despite their status as mercenaries and hence expendable and purchasable killers. In one of the first scenes of The Expendables, Gunnar, who is under the influence and wants to hang one of the just seized pirates, is told by Barney "This is not how we work" (Stallone, 2010, p. 06:45). In the continuation of the scene, it becomes clear that although their work is characterised by extreme violence, the Expendables only kill when they are attacked and not for enjoyment or pure revenge since this is seen as "sick" behaviour (Stallone, 2010, p. 06:55). Their work ethic and honourability are further staged by contrasting them with the respective opponents in the films: all antagonists have no moral values and are driven solely by monetary motives, greed and narcissism.

Emphasising the moral superiority of the protagonists and the significance of their occupation is essential in the characterisation of the protagonists since it justifies their brutality and aggression whilst stressing over and over again that the main characters are embodiments of true masculinity (Boyle \& Brayton, 2012; Feasey, 2011). This enables them, and only them, to save the world from evil. Their representation as driven by a high level of morality and the desire to fight injustice and to support the disadvantaged also sets the stage for another crucial element of masculinity: control (Beynon, 2001). In contrast to the antagonists, the actions of the Expendables team's professionalism and noble aims empowers them to stay focussed and in control even in times of highest emotional turmoil. The team's enemies are ruled by irrational greed for power and money, an unrealistic feeling of invincibility and superiority, which subsequently leads them to, first, lose control of themselves and then of the battle. The Expendables, on the other hand, hardly ever lose composure. In fact, the harder they are beaten, in the literal and metaphorical sense, the calmer and more controlled they become even when feeling extreme rage and deep sadness.

Maggie: 'Do you think about the young man who died?'

Barney: 'All the time.'

Maggie: 'You don't talk about him much.'

Barney: 'No, that's how we deal with death. Can't change what it is, so we keep it light until it's time to get dark. And then we get pitch black, understand?'

(West 2012, 51:26-51:42)

The apparent high moral stand the Expendables claim is therefore inextricably linked to control - one of the main criteria of hegemonic masculinity. In other words, although the portrayal of Barney and his crew appears to offer a counter-narrative to hegemonic masculinity scrips, their depiction, in fact, mainly rests on crucial aspects linked to dominant 
understandings of masculinity such as displaying and being in control and having a profession of crucial significance for society (Bourdieu, 2001; Cheng, 1999; Connell, 2005; Pyke, 1996).

Since hegemonic masculinity is mostly dependent on the domination and submission of others and as such a highly relational concept (Beynon, 2001; Bourdieu, 2001; Connell \& Messerschmidt, 2005; Fausto-Sterling, 1997), it is of crucial relevance to explore the portrayal of the supporting cast to identify how their dramaturgical construction facilitates images of manhood in the films. As can be seen in the quote above, supporting actors, particularly women but also men, in The Expendables trilogy are often used to emphasise a masculinity ideal which is, after all, much less unconventional and outdated than it might seem at first sight (Chivers, 2011). Yet, the members of the team do not represent the current hegemonic understanding of masculinity of being white, affluent, heterosexual and powerful and middle-aged (Cheng, 1999). Indeed they are portrayed as being somewhat socially outcast working-class men even though the different nationalities and races and the high educational level of some of the characters deviate from the conventional definition of working-class men (Banet-Weiser, 2014; Calasanti \& King, 2005). The social setting and mentality of the team are set in stark contrast to men such as Church, their employer in The Expendables and The Expendables 2, and Drummer, their client in The Expendables 3 - CIA bureaucrats who are not willing to risk their own life and reputation but have the power to hire men (and occasionally women) to do what is necessary without dirtying their own hands. As such they resemble the hegemonic masculinity ideal which becomes crystal clear when Barney in The Expendables 2 says to Church:

You know Church, you're the kind of guy that pulls strings, and everyone else does your dirty work. Because you never had the guts to do it yourself.

(West, 2012, p. 41:54)

This is a crucial narrative construction since it firmly establishes the seemingly long outdated and anachronistic masculinity codex strongly linked to working-class men as a viable alternative to dominant views of masculinity whilst questioning the right of pseudo-pacified and more politically correct men to patriarchal dominance (Connell, 2009; Coston \& Kimmel, 2012; Hall, 2000). This is further underlined when both Church and Drummer, finally join the action and support the team in their effort to save the world.

Particularly noteworthy within this context is the representation of women in the film trilogy. In general and genre-typical, female characters are a rare phenomenon, archetypically much younger than most male protagonists and outstandingly attractive in all three films (Lauzen, 2015; Tasker, 2012). When they appear, they are either portrayed as needing male protection and rescue and as such motivate the heroes' actions which, in turn, further stresses the 
manliness of the protagonists or they are part of the team and are resultantly and politically correctly staged as being on par with their male counterparts (Dutt, 2014; Holmlund, 2002). Interestingly, only the first part of the film trilogy follows genre conventions typical for the depiction of women in the hard-boiled action films of the 80s (Mulvey, 1992 [1975]; Tasker, 2012). Lacey, Chris Christmas' (Jason Statham) former girlfriend, needs him to rescue her from the abusive and violent relationship with her new boyfriend, whilst Cheyenne, a girl Tool (Mickey Rourke) has apparently just met, is staged as merely an accessory of Tool to emphasise his masculinity and heterosexuality.

When Tool rides into his workshop on his Harley with the sexily dressed and very young Cheyenne on the back seat, the subsequent scenes appear to be a manifestation of what Laura Mulvey famously defined as "the male gaze"(Mulvey, 1992 [1975], p. 11). When he cannot seem to remember her name, when he asks her to go upstairs, fix him a drink and wait for him until he has finished his business (tattooing and chatting with Barney) and when he finally sends her off with a slap on her backside, and her long naked legs slowly move up in the elevator in the background, it is undoubtedly repulsive and hard to watch, especially for women. However, in my view, it also highlights that Tool is a relic of the past who cannot really be taken seriously but must instead be seen as an ironic reminiscence of a view and treatment of women long outdated. The whole scene finds its climax when Tool says:

I don't want to die alone. I want to die for something that counts. I want to die in the arms of a woman - not with a woman. I want to die with somebody who cares about me. I don't want to die for a woman. I want to die next to a woman (Stallone, 2010,17:11-17:23).

Lauren Jones in her role as Cheyenne and Charisma Carpenter as Lacey are objects of the male gaze and they are contextualised as passive and as depending on men for support (financial or emotional) and protection. The inclusion of the scenes revolving around Lauren and Cheyenne in the first part of The Expendables are, thus, used to underline the manliness of the male cast while firmly establishing heteronormativity as the socio-cultural standard of male and female behaviour (Hills, 1999; Mulvey, 1992 [1975]).

Both women are introduced in two successive scenes which highlight the problematic relationship status of the protagonists and aids the construction of masculinity characterised by the inability to express positive emotions whilst longing for emotional stability and support. In other words, the scenes facilitate a rather popular picture of a bad-boy masculinity in which men are portrayed as hard on the outside but soft inside who need women as a safe haven to regain strengths for their dangerous but most significant job (Kimmel, 2012; King, 2007; Tasker, 1993b). This is an old-fashioned and nowadays extremely unrealistic image of heteronormativity as socio-culturally prescribed standard for relationships and linked gendered separation between the public and private sphere which significantly facilitated the 
establishment of the strictly hierarchical respectively patriarchal gender system current western society is still majorly influenced by (Bourdieu, 2001; Fausto-Sterling, 1997, 2000). However, after The Expendables Tool vanishes from the cast and is never seen again and the characterisation of women in the two follow-ups comply with a more modern and politically correct contextualisation of femininity which had already been established in the character of Sandra in the first film.

Sandra in The Expendables, Maggie in The Expendables 2 and Luna in The Expendables 3 are shaped after the heroines Ripley in Alien, Lara Croft or Sarah Connor in Terminator. They are presented as resourceful, active and physically as well as mentally strong and young heroines whose muscularity and behaviour match and often outperform their male counterparts' (Tasker 2002, 1993b). It is likely that this more acceptable depiction of women is owed to targeting female audiences, but the contextualisation of these modern heroines also reflects a changing cultural narrative of femininity and masculinity (Brown, 2004; Inness, 2004).

I believe that the portrayal of women in The Expendables trilogy is significantly informed by neoliberal ideology - an ideology in which the value of the individual is determined by his or her productivity and consumption power (Gergen and Gergen 2000, Chomsky 1999). As such, the formerly genre-typical binary of active men versus passive women is overwritten by a storyline transmitting that gender, age, social class or race are insignificant if one is productive and useful to neoliberal society (Cruikshank 2013, Gergen and Gergen 2000).

Notwithstanding this, it is striking that, besides the fact that women are a rarity, underrepresented and much younger than most of the male protagonists, even these strong and active heroines are construed differently to their male counterparts in the films. It is the women who show emotions and who display interest in and care for the male leads' feelings. Simultaneously, the male team members seem to feel particularly protective towards the female team members and treat them with unusual respect and chivalry. Particularly Barney's behaviour is characterised by veering between fatherly consideration and sexual attraction which becomes apparent in the following quotes:

Barney: (handing over a folded piece of paper) The second account number's mine -

Maybe it'll help get things going.

Sandra: No, I can't. You've done so much.

Barney: So have you - A gift for a gift - Take it.

Sandra: Will you come back ever?

Barney: Can't say, but I promise you'll always be near me.

Sandra: 'Tienes que encontrar un mujer que siempre se quedara despierto solo para verte dormir' - Someday you will find a woman who'll be happy just to watch you sleep. 
Maggie: I don't have to go. If you want me to stay, I'll stay. And I did save the babysitter's life.

Barney: That you did do.

Maggie: Maybe I'm good luck for you.

Barney: But I could be bad luck for you. And I can't allow that.

Maggie: If you ever want help, call me. Or your dead.

Barney: I'll do that.

(West, 2012, 01:25:45-01:26:12)

Luna: Just wanted to say thanks.

Barney: For what?

Luna: For caring.

Barney: Oh, come on.

Luna: You know, if you were 30 years younger ...

Barney: I'd be afraid of you.

(Hughes, 2014, 01:52:00-01:53:26)

It is also noteworthy that although all three women, Sandra, Maggie and Luna, can clearly carry their own weight, only Maggie is not shown in a scene (or several scenes in the case of Sandra) in which they need saving by Barney or another team member. The depiction of women in The Expendables trilogy is, therefore far from gender-neutral and based on rather conventional understandings of gender differences (Negra et al., 2007). Women are portrayed as more compassionate and sensitive than the male cast which elicits the male protective instinct whilst the fact that they possess similar abilities to the male protagonists is contextualised as a somewhat surprising and kind of unnatural and therefore intimidating occurrence (see above: Barney's reply to Luna: "I'd be afraid of you.") (Butler, 2011; Fausto-Sterling, 2000; Schippers, 2007). As such male attributes such as strengths, dominance and control are presented as the measure of all things against which female performance and character are judged (Schippers, 2007). Resultantly, all three films insist and perpetuate a stereotypical and patriarchal view of gender difference which supports the hegemony of men.

All in all, the trilogy communicates a narrative of masculinity which, even if exaggerated and, at times, self-deprecating, is essentially based on the current constituents of hegemonic masculinity. The members of the Expendables team find identity, recognition and selfrespect exclusively in and through the extraordinary meaningfulness of their work and the high efficiency and moral superiority they display when executing the respective mission (Calasanti \& King, 2015). The good guys can only defeat their enemies by staying in full mental, emotional and physical control of themselves and everything and everybody surrounding them and by being and acting entirely autonomously from the state, their employer, but also, and most importantly, from any politically correct understanding of a pseudo-pacified masculinity (Chivers, 2011; Edwards, 2004; Hall, 2002; Mosse, 1998). An 
essential criteria in establishing the hegemonic status of the Expendables team entails proving and displaying their dominance over other men (bureaucrats, bad guys and pussies) and women or by allowing them to join the ranks of the team once they have, in the case of other men, recovered their true manliness or, in the case of women, proven that they are as manly as any of the team (Bourdieu, 2001). As such, The Expendables are not constructed as an alternative to the current view of hegemonic masculinity but much rather as representing the true nature of men unhampered by the corset of current socio-cultural frames (Boyle \& Brayton, 2012). Nonetheless, the older age of the main cast and the often negative connotations linked to ageing seriously impede their representation as the embodiment of hegemonic masculinity. The natural decline in physical and mental abilities cannot be entirely ignored in a storyline which rests on the dissemination of the protagonists as representing true and biologically determined masculinity without losing credibility (Feasey, 2011; Thompson, 2006).

\subsection{Narratives of Male Ageing in The Expendables Trilogy}

As discussed repeatedly throughout my thesis, current hegemonic narratives of ageing unfold within two contradictory socio-cultural frames, namely ageing as decline versus successful ageing (Gullette Morganroth, 2004). The Expendables trilogy utilises both concepts of ageing in the portrayal of the older protagonists. However, the genre of action cinema as well as the on- and off-screen personae of the main cast naturally determine a predominant focus on the narrative of successful ageing. In fact, in many respects, all three films can be read as a blueprint for how to age successfully as a man (Davey \& Glasgow, 2006; Phoenix \& Griffin, 2015; van Dyk, 2014).

Personal responsibility and resulting autonomy and independence from friends, family and the state, activity which ideally benefits society and consumption are crucial prerequisites for ageing successfully (Sandberg, 2013; Segal, 2014; Woodward, 2006). The Expendables team and particularly the oldest members of the team, Barney, Trench or Tool are in many ways embodiments of the successful ageing ideal. Although their faces and hands (Bordo, 2004; Dolan, 2013; Dolan \& Tincknell, 2012; Fairclough, 2012) and the use of reading glasses are indicators of their older age, their actions and behaviour quickly lay to rest any suspicions that their age might have reduced their mental and physical capabilities. Even if only jokingly and casually mentioned, any reference to an age-related reduction of the older protagonists' capabilities is immediately counteracted by either showing them in high-speed and extraordinarily violent action scenes in which they match and often outperform their antagonists and often also the younger cast or in scenes in which they calmly, wisely and, 
most importantly, rationally make decisions on how to further proceed. Indeed, it seems as if their older age has increased not only their physical strengths and agility but also as if it has enabled them to manage their emotional landscape better than the younger teammates which subsequently supports their efficiency (Chivers, 2011; Cruikshank, 2013; Jackson, 2016).

Linking older age with senescence and wisdom is not unusual in western cultural settings. "Throughout the history of western thought, wisdom has been associated with the coolness of reason and evenness of judgement, with detachment and balance" (Woodward, 2003)58 In societies which hold emotional control and rationality in such high esteem this definition is often hailed as supporting a positive view of ageing since it is one of the rare positively connoted characteristics related to increasing age which is not part of the successful ageing paradigm and therefore does not demand of the older individual to stay youthful, independent and productive. Using one's wisdom to contribute to society is, therefore, one of the few meaningful social roles available for all older people in contemporary western societies, regardless of, at least, their level of physical fitness and mobility, and as such it could facilitate the positive self-perception of an older person (Cruikshank, 2013; Scheibe, Kunzmann, \& Baltes, 2007).

Nonetheless, as succinctly outlined by Kathleen Woodward (2003), wisdom as an attribute of older age is a double-edged sword as its conceptualisation also communicates a certain level of emotional distance and aloofness from matters which seemed relevant at earlier stages of one's life. It hence represents yet another template for older age which by linking older age to disengagement and cognitive and emotional control relegates the older population to the side-lines of society where they are distant observers whilst setting another idealised, homogenising and nearly unachievable standard for evaluating older people's success in ageing (Cruikshank, 2013; Gullette Morganroth, 2011). As such "wisdom as an ideal ...often serves as a screen for ageism."(Woodward, 2003)56. Interestingly, the portrayal of the older members of The Expendables team, and particularly of Barney, appear, however, to draw on both narratives, the narrative of wisdom as a natural outcome of old age and the narrative of successful ageing as staying autonomous, active and youthful. As such, their depiction could be viewed as representing a harmonious combination of being wise and actively contributing to society (Woodward, 1988). Linking these two seemingly contrary conceptions of older age is essentially enabled and dependant on the age atypical physical and mental fitness of the protagonists.

At no point in any of the films can there be a doubt that the older protagonists in The Expendables trilogy are in full control of their hypermusclar and outstandingly fit and agile bodies and that neither their physical nor their mental potential has decreased as a result of 
their older age. As such they are living proof of the possibility to control the ageing process provided one is determined, strong-willed and disciplined enough (Carroll \& Bartlett, 2015; Featherstone \& Wernick, 1995; Gilleard \& Higgs, 2000). That The Expendables team routinely and successfully saves the world from destruction, that they put their lives on the line and that they have taken it on themselves to cleanse the earth from very evil people, emphasises the significance of their occupation and subsequently determines their high value for society (Cruikshank, 2013).

At the beginning of each film, the team and their work are contextualised as underestimated, as social outcasts and as stuck in a historic time long gone with moral principles not suitable for modern times (Collinson, 2010; Hall, 2000, 2002). This becomes clear in the following scene, which stands representative for numerous similar dialogues in the three films:

Stonebanks: It's a dark business Barney, don't pretend you're not in it. The baddest survive, those are the rules, I didn't make them up. You see Barney here was content to work for the small end money. As an employee. Now being boss is more lucrative, but that's a concept that somehow eluded you. Plus you got that pesky moral conscience.

God that stuff gets in the way. He thinks he's the good guy.

Barney: Keep talking while you can.

Stonebanks: Sure, you got a conscience that makes you weak. Success, real success... is being willing to do the things that other people are not.

Barney: Not everyone's as sick as you.

(Hughes, 2014, 01:05:27-01:05:55)

The humiliating way Barney and his team are treated by their employers and the antagonists is hard to watch. Although the narrative construction of the action hero as a rebel fighting alone and against all the odds is typical for the genre (Tasker, 1993b), the continuous reference to the outdatedness of Barney's mentality is linked tightly to the chronological age of the main cast. The films thus reflect current views of ageing as characterised by not being able to adjust to the requirements and speed of modern society (Baars, 2015).

This is a particularly clever narrative manoeuvre since when the team finally and after much hardship triumph over their enemies and when they converted men embodying the current standards of hegemonic masculinity to join their ranks, this is not just a triumph of good over evil but also a victory of older age and related anachronistic understanding of manliness over current views of ageing as decline and a tamed and socially acceptable version of masculinity (Carroll \& Bartlett, 2015; Feasey, 2011). Essentially the films, hence, communicate that it is not the older age of men which impedes their virility and related claims to power but the restraint and domestication of a naturally, unruly and wild macho masculinity in modern hegemonic constructions of masculinity (Boyle \& Brayton, 2012; Hall, 2002; Lennard, 2014). 
This contextualisation is further underlined by the mise en scene. On the one hand, the older protagonists of the films are surrounded by black-and-white photos, classic motorbikes and cars, a battered water plane, placed in an old army base resembling the US in the 50's, or in nostalgic scenes which pay tribute to the shoot-outs and the general narrative construction of masculinity in westerns of the $60 \mathrm{~s}$ and $70 \mathrm{~s}$. On the other hand, the overwhelming hightech armoury and technology used underline that the team is far from being dinosaurs in a modern world ruled by corporate and often younger men (Hearn 1995) who represent the current ideal of masculinity. Simultaneously, it stresses their value as consumers of goods which support but do not enable their efficiency (Calasanti \& King, 2005, 2015).

However, in accordance with the narrative of successful ageing, it is of crucial importance to establish that battling age is not an easy endeavour. Only through will power, discipline and strength of character can the decline of ageing be conquered - a narrative which is firmly based on linking the individual motivation and responsibility to stay fit, healthy and youthful to moral goodness increasingly communicated in current neoliberal society (Calasanti \& Slevin, 2001; Gilleard \& Higgs, 2000). To highlight the high morale and bravery of the team, the films, therefore, combine scenes in which the team perform at the highest level possible with scenes in which they battle with their own demons resulting from having lived a life full of violence, destruction, grave danger and loss. These demons, however, are grounded, by and large, on notions linked to ageing understood as decline (Chivers, 2011).

In all three films positively connotated attributes of ageing such as wisdom, serenity and emotional stability are skilfully interconnected with much more negatively connotated aspects such as cynicism, resignation, bitterness and loneliness predominantly associated with bad old age (Cruikshank, 2013; Woodward, 2006). Particularly Barney and, to a certain degree, Tool in the first film, are staged as ambiguous characters continuously balancing on the edge between good and evil. They are in constant danger of losing the fight against their dark side and of giving up the futile battle for justice in a corrupt world. Indeed, the motif of 'dark' and 'black' recurs several times in the films and is clearly associated not only with a total lack of morality but also with death - the ultimate and unavoidable threat to masculine control as evident in the following dialogues:

Christmas: 'This new job sounds bad.'

Barney: 'Yeah, but it'll pay good.'

Christmas: 'Yeah well, money aside, what happened to your code?'

Barney: 'What code is that?'

Christmas: 'The target has to deserve it.'

Barney: 'Oh, that was cancelled due to lack of interest.'

Christmas: 'You're dark, dark and cold.'

Barney: 'You noticed!?'

Christmas: 'And I ain't buying into it, by the way.'

Barney: 'You will.' (Christmas leaves) 
'Stick around young man, you will.'

(Stallone 2010, 24:54-25:15)

Tool: '... Kinda feelin' like... dead too, ya know? My heads all very, very black place. Didn't believe in shit. Just goddamn Dracula black...'

(Stallone 2010, 57:55)

Maggie: 'Do you think about the young man who died?'

Barney: 'All the time.'

Maggie: 'You don't talk about him much.'

Barney: 'No, that's how we deal with death. Can't change what it is, so we keep it light until it's time to get dark. And then we get pitch black, understand?'

(West 2012, 51:26-51:42)

Barney: You went dark.

Stonebanks: It's a dark business Barney, don't pretend you're not in it.

(Hughes, 2014, 01:05:27)

However, surrender and resignation deprive men of an occupation which provides meaningfulness, direction and relevance in their lives regardless of age (Hearn, 2014; King, 2007; Price \& Livsey, 2015). This can subsequently only lead to either loneliness, and the resulting depression and/or a pathological obsession with irrelevant and ultimately selfdestructive values as the antagonists and, to a lesser degree, Tool and Gunnar most impressively prove.

Typically for the genre of action films except for Christmas and Hale Caesar, the team do not seem to have any conventional relationships to spouses, family or friends, apart from the Expendables. Instead from the first until the last part of The Expendables the team is staged as a surrogate family in which the members are held together by pursuing a mission, by sharing the same values and generally by the life they live. The close relationship to the other team members seemingly extends beyond age, race and also gender and it is here where the team receives recognition, respect, human warmth, where they feel safe and where everyone cares about the other (Boyle and Brayton 2012). However, lack of rationality or efficiency and 'giving in to the dark side' lead to immediate exclusion from this intimate circle of friends and comrades-in-arms and resultantly to the condemnation of the excluded to a life of loneliness, insignificance, purposelessness and ultimately death (Calasanti \& King, 2015; Clarke \& Bennett, 2015; Kimmel, 2001). This is illustrated by the conceptualisation of Gunnar who is excluded from the Expendables team because of his drug addiction and subsequent irrational and uncontrollable behaviour, then joins the dark side of their enemies, nearly dies in a fight with Barney but refined and healed from drug addiction is allowed to re-join the team by the end of part one of The Expendables. 
The strong emphasis on teamwork, loyalty, trust and cooperation as the basis for reaching the aim of their respective mission is unusual for the genre of action films in which the hero usually seems to stand all alone against an overwhelming enemy (Tasker, 1993b). Although often joined by others, often women, this is more accidental and frequently unwanted cooperation. In general, action heroes predominantly match the 'lone fighter' image - an image which finds its reflection in the character of Booker, whose nickname quite tellingly is 'Lone Wolfe'. He nonetheless joins the team and, in the end, utters "it is sometimes fun to run with the pack" (West 2012, 86:16). The same is true for Trench who initially works alone and is a clear competitor of Barney and his team but he not only, in the course of the other two parts, becomes an unofficial part of the team, but he is also joined by an original member of the Expendables, Ying Yang (Jet Li).

Conceptualising teamwork as the crucial element for overcoming any seemingly insurmountable obstacle is, in my view, neither an indication for the waning power of the older protagonists nor is it based on a socialist view of equality but it is much rather a crucial element of the conceptualisation of " "real' men whose loyalty to one another endures beyond their physical bodies" (Boyle \& Brayton, 2012, p. 475). The homosocial bond the members of the team share which can even, although to a lesser extent, include women if they have proven their 'manliness', is construed as outshining all other relationships since it is based on deep trust which within the confinements of the team, permits the protagonists to act out their true nature without having to comply with hegemonic standards of behaviour. This becomes particularly clear in the scenes in which Christmas' rocky relationship is the topic of discussion between Barney and Christmas. In Barney's view, "the man who gets along best with women is the man who gets along without them" (Stallone 2010, 34:24). Despite the fact that the general mistrust in women is in line with genre customs (Tasker 1993a), his remark also hints at a strong scepticism towards any type of dependency on anybody or anything except the team. Once again the film's narrative here communicates that a loss of autonomy due to emotional or physical dependency can only lead to disaster whilst conceding to the need for interhuman connection and solidarity which, however, can only be found in true male friendship and very occasionally perhaps with some women who have proven worthy of male trust (Featherstone \& Hepworth, 2005).

Nonetheless, the Expendables are not a pari passu and unorderly pack of expendables killers drawn together by a similar mindset and jobs to be done. In fact, the team is ordered strictly hierarchical and resembles a military organisation. It is led by Barney whose authority surpasses any struggles and upset in the team or with decisions Barney has taken. He is clearly staged as a fatherly figure who is strict but cares immensely about his team and their well-being. This particularly true with respect to the younger members of the team. Whilst 
in the first film no youngsters are included in the team, in the second and third film intergenerational cooperation takes up a large part of the narrative construction, and it is within this context that older age is discussed most frequently.

In general, the storyline in all three films is based on a hegemonic socio-cultural narrative which assigns a higher value to the life of younger people than that of older people since the young not only have their life still ahead of them but also since they are considered to be entirely innocent and free of any guilt which could justify their assassination (Gullette Morganroth, 2004, 2011). It is this understanding which is clearly communicated when after the violent death of Billy the Kid Barney says: "Why is it that the one of us who wants to live the most, deserves to live the most, dies. And the ones that deserve to die,...keep on living? What is the message in that?" (West, 2012, 40:19). Indeed, it is striking that Barney's suicidal missions are frequently motivated by the intention to save the younger such as Sandra in the first film or the new youngster team in the third film or to retaliate their death in the second film. Besides increasing Barney's and the older team's likability and emphasising their integrity, this is, however also a clear message sent to anyone who views the older protagonists as belonging in the scrap heap (Chivers, 2011).

All films quite clearly communicate that, in fact, it is the younger who need the older and not the other way round. Comments such as when Barney says at the sight of a new but battered water plane: "That thing belongs in a museum" and Trench answers: "We all do."(West, 2012, 87:01) or when Barney tells his team: "We're not the future anymore, unfortunately for us, we're part of the past."(Hughes, 2014, 30:15) or when he says: "It's a young man's game.'(Hughes, 2014, 40:30) are immediately disproven by the continuation of the story. As a result, The Expendables trilogy presents intergenerational collaboration as possible and as mutually beneficial (Gullette Morganroth, 2004; Jennings \& Krainitzki, 2015). This is in contrast to public representations informed by a socio-cultural view which Susan Hamad calls "toxic intergenerationally" (Gill et al., 2016; Hamad, 2013) which precludes a fruitful cooperation of different generations since it is "characterised by conflict and divisions that can't be surmounted" (Gill et al., 2016)3. Nonetheless, the films also transmit that alliances between generations only work on the basis of a clear hierarchical and quite conventional structure in which the older have the say and are on top of the packing order whilst the younger need to respect and learn from the older protagonists even if in certain areas such as technological advances they might be more knowledgeable (Calasanti \& King, 2005).

The Expendables trilogy is thus based on both hegemonic narratives of ageing: ageing as decline and successful ageing. Notions of ageing as decline are contextualised as the result of giving in to 'the dark side'. In other words, losing moral integrity will unavoidably lead 
to the exclusion from the privileged and strictly hierarchically ordered circle of true men who have taken it on them to save the world. This exclusion must be viewed as the ultimate punishment for a lack of will power and discipline which, as the films insist, necessarily ends in social isolation, depression and death. This narrative reflects a socio-cultural understanding which links health, fitness and the will to stay youthful to moral goodness and superiority (Clarke \& Bennett, 2015; Hearn, 2014; Hearn \& Sandberg, 2009).

The construction of ageing as decline is therefore used to underline the high significance of personal autonomy while acknowledging that human beings, even men, need social recognition, warmth and support. Without risking personal autonomy and emotional and/or physical dependence, this substantial sociality can, however, only be found in a male camaraderie grounded in trust, loyalty and in performing tasks that are not only meaningful for the individual but benefit society as a whole. The high significance of what the Expendables team do within the frame of an organisation ordered in military-style gives the members of this exclusive circle direction, increases their social recognition and value and, most importantly, it can, to a certain extent, enable them to move beyond intersectional discriminators such as age, gender, race or social background (Gilleard \& Higgs, 2000). The films, therefore, transmit a socio-cultural understanding of ageing, which utilizes both hegemonic narratives of ageing - decline and progress. As such they promote and emphasise a construction of masculinity rooted in a seemingly outdated ideal of masculinity in which control and dominance are established by explicit violence, brutality and the submission of anybody who does not adhere to these patriarchal standards and which is justified by the moral superiority of the men, and very rarely women, who execute it (Chivers, 2011). The Expendables trilogy hence disseminates a somewhat reactionary perception of masculinity one has to be wary of since it purports a practice of patriarchy which is essentially based on propagating the ruthless marginalisation, discrimination and subordination of anyone who is not part of the elite circle of male hegemony (Bourdieu, 2001). Nonetheless, the films also offer critical insights into the current hegemonic constructions of ageing and masculinity.

\subsection{Neoliberalism \& the Construction of Male Ageing in The Expendables Trilogy $^{1}$}

As discussed in chapter four of this thesis, today hegemonic masculinity status is closely tied to being white, heterosexual, middle to upper-class, Christian and young to middle-aged

\footnotetext{
${ }^{1}$ Parts of this section have been adapted from Bühring, L.-N. (2017). Men Refusing to Be Marginalised Aged Tough Guys in The Expendables and The Expendables 2. Journal of Extreme Anthropology. 1(3). 1-20.
} 
(Cheng, 1999; Connell, 2005). Notwithstanding this, hegemonic masculinity is a contextbound and highly relational construction, and as such, at any specific time in history and within different socio-cultural settings there are multiple understandings of masculinity of which one is usually hegemonic while the others are marginalised and strive to replace this dominant masculinity ideal (Coston \& Kimmel, 2012; Kimmel, 2001). Particularly in times of social, political and/or economic upheaval due to, for instance, advances in technology and linked alterations in the social and political environment as is characteristic for the past twenty years or the strong civil liberty movements in the $60 \mathrm{~s}$ and $70 \mathrm{~s}$, are especially prone to instigating alterations in hegemonic narratives (Negra et al., 2007). When one's belief and value system is questioned and alterations to it are imminent, it leaves individuals in any respective society feeling insecure and disoriented which often leads to a nostalgic idealisation of former times and their seemingly clear-cut socio-cultural frames (Kimmel, 2001).

The increasing emancipation of women and the continuous social and political struggle for equality has, in the past thirty years, resulted in some confusion about gender roles. Public discourses on the so-called crisis of masculinity in the closing years of the $20^{\text {th }}$ and the beginning years of the $21^{\text {st }}$ century lament the loss of conventional and binary gender constructions. Linked was the perceived emasculation of men which does not reflect the seemingly natural order although this natural an order is, at least in my view, neither natural nor standardisable but merely a reflection of the desire to secure patriarchal privilege (Allen et al., 2015; Baur \& Luedtke, 2008; Bourdieu, 2001; Cornwall et al., 2016).

Within this context, it is not overly surprising that films like The Expendables trilogy in which an old-fashioned masculinity script thought to be long overcome is revived were internationally highly successful since the masculinity frames presented in the three films are so much less ambiguous and confusing than current understandings of masculinity. They thus leave much less room for insecurity and confusion over one's own gendered identity (Butler, 2011; Fausto-Sterling, 1997, 2000). That the older protagonists of the films grew up in a time and social context in which precisely the masculinity construction they communicate was dominant increases the authenticity and believability of the filmic narratives (Connell, 1993; Hall, 2002). The older age of the protagonists, therefore, provides an essential basis for allowing audiences to accept the ultra-conservative and politically largely incorrect understanding of masculinity perpetuated in the films - after all, being stuck in the past is the privilege of older people, isn't it?

There can be no doubt that the older age of the majority of the cast in The Expendables, their social background and the macho-masculinity they transmit today represent a marginalised construction of masculinity which, however, is clearly construed as a viable alternative to 
the current hegemonic understanding of masculinity. To establish the rule of right of this view of masculinity the films utilise a strategy which in Coston and Kimmel's (2012) application of Goffman's approach to stigmatisation (2009) is typical for handling marginalisation, namely militant chauvinism. This is a strategy in which differences to the ruling ideal are stressed and used to establish the superiority of the marginalised group. The Expendables trilogy can, therefore, also be read as a manifest and outcry of older, workingclass men not willing to accept their dismissal (Donnar, 2016). The films are a proclamation of their superiority over the competing masculinity ideal of corporate masculinity, and they clearly communicate that to save the world from everything going wrong it needs men like the Expendables. The films, hence, criticise nothing less than the socio-economic system of neoliberal financial capitalism, which has produced the current dominant masculinity ideal (Griffin, 2005; Kotz, 2002; Pyke, 1996).

Barney articulates this criticism of the currently trending idea of masculinity openly in The Expendables 2:

'You know Church, you're the kind of guy that pulls strings, and everyone else does your dirty work. Because you never had the guts to do it yourself' (West, 2012, 41:54).

The name 'Church' and the selection of a church for the first meeting of Barney and Church supports the interpretation that 'Church' acts in the name of and as a secular assistant to the new godlike idols of neoliberalism: money and power. The Expendables are his crusaders whose job is to protect and preserve neoliberalism, the economic and political system which supposedly has proven to be without alternative (Chomsky 1999). Having somehow exceeded their expiry date, these musclemen now perform and employ their masculinity in the service of the hegemonic group of men such as Church or Stonebanks in the films or of film producers in the real world (Connell, 2009; Hall, 2000; Pyke, 1996).

Church and later Drummer delegate the necessary, but less valued and extremely dangerous aspects of manliness, to Barney and his crew who in his place execute what needs to be done (Kuldova, 2014, 2016). By doing so these men satisfy still widely accepted standards and rituals of masculinity but without risking their own privileged life (Hall, 2002; Pyke, 1996). Presenting Church in The Expendables and The Expendables 2 and Drummer in The Expendables 3 as bureaucratic weaklings, who do not do much more than paper-pushing but also stand representative for a dominant masculinity construction, is used to further underline the true manliness of Barney and his team (Coston \& Kimmel, 2012). More importantly, however, when Church and Drummer, provoked and purged by Barney's remark, later in the narratives reclaim their true manliness by joining the battle, this marks a victory of marginalised masculinity over the new whitewashed masculinity ideal and a defeat of neoliberal ideology (Boyle \& Brayton, 2012; Feasey, 2011; Lennard, 2014). 
This criticism of the neoliberal understanding of masculinity is also transparent in The Expendables when Vilena is portrayed as an island which has been infected by the evils of neoliberalism and is close to complete destruction by exploitation (Harvey, 2007b). It, therefore, does not surprise when General Garza says: "We will kill this American disease!" (Stallone, 2010, 87:39) but it is also a clear and explicit declaration of war against neoliberalism in a U.S. American action film. In The Expendables 2, ideology criticism is less pronounced and replaced mainly by elements of humour and satire. However, the antagonist Villain in The Expendables 2 is once again like his predecessor in The Expendables, Monroe, motivated solely by greed for money and power and characterised as bare of any morals or human traits. In The Expendables 3 the storyline is much more personal and everything circles around Stonebanks, a former Expendables member who has turned bad and who is, therefore, on literally every level, much worse than Villain. He again is staged as the personification of everything which characterises the dark side of neoliberalism namely amorality, greed and a total lack of solidarity or empathy for fellow human beings (Katz \& Marshall, 2003; Žižek, 2009). This becomes more than evident when he, in a conversation with Barney, says:

Stonebanks: It's a dark business Barney, don't pretend you're not in it. The baddest survive, those are the rules, I didn't make them up. You see Barney here was content to work for the small end money. As an employee. Now being boss is more lucrative, but that's a concept that somehow eluded you. Plus, you got that pesky moral conscience. God that stuff gets in the way. He thinks he's the good guy.

Barney: Keep talking while you can.

Stonebanks: Sure, you got a conscience that makes you weak. Success, real success... is being willing to do the things that other people are not.

(Hughes, 2014, 95:27-96:10)

Both men and their actions are hence presented as the embodiment of a socio-economic system that values profit and productivity above everything else (Chomsky, 1999; Featherstone \& Wernick, 1995; Harvey, 2007a).

The ideology criticism in The Expendables trilogy is undoubtedly partly owed to the genretypical staging of the protagonist against the state or the bureaucracy linked to the state which underlines his image of being a true man (Tasker, 1993b). However, in my view, the ideology criticism of The Expendables is too blunt and explicit to be only attributed to genre conventions. In fact, the stories would have worked just as well if, instead of neoliberalism being the target of the narrative, another ideological system had been chosen as public enemy.

I believe that the films' insistence on contextualising the perversions of a neoliberal system must be seen as a critical reflection on a society in which men such as the Expendables do 
not have a place anymore. In other words, in a system which worships youth, efficiency and consumption above everything else, in which not only products and services but also human beings are evaluated on the basis of these market values, individuals who do not fit these criteria are literally expendable and thus excluded and marginalised (Calasanti \& King, 2005; Katz \& Calasanti, 2014). This value system affects the Expendables team in the films as well as in the real world since they are a dying out species in the imaginary world of the films as well as in the real world of the Hollywood film industry (Donnar, 2016; Treme \& Craig, 2013). As actors who rely on their hard-boiled bodies for employment, ageing and changes in the cultural understanding of masculinity threaten their professional careers in an industry which is obsessed with youth, efficiency and productivity and which is highly dependent on sensing and following current cultural standards (Addison, 2006; Boyle \& Brayton, 2012; Chivers, 2011).

At the same time, however, this seemingly out of fashion roughness and toughness, physical power and aggression of the main cast aid the hegemonic group of men in maintaining their patriarchal dominance (Hall, 2002). Their extraordinary strength and muscularity in combination with their actions and behaviour seem to prove and legitimate the dominance of heterosexual men - a belief which is still one of the cornerstones of a neoliberal ideology presented as a seemingly natural order which benefits all (Bourdieu, 2001; Butler, 2011; Chomsky, 1999). As such, and perhaps partly unwillingly, the films are characterised by the ambiguity of, on the one hand, communicating and thereby enforcing hegemonic sociocultural narratives of ageing and masculinity while, on the other hand, questioning and critically reflecting on the socio-economic structure these hegemonic cultural narratives are informed by.

This ambiguity becomes particularly apparent in the use of self-deprecating humour and extreme exaggeration of action and devastation in the trilogy. The sheer amount and size of muscles, motorbikes, corpses and general destruction in the three films is so much over the top that one cannot help but be amused. While The Expendables is still characterised by a more serious undertone which includes elements of film noir, the other two films in parts veer towards an action-comedy. Humorous scenes are not just established by reference to the earlier performances of Trench (Arnold Schwarzenegger) in the Terminator series but particularly by playing on the hyper-masculine and hyper-muscular image of the actors. Two of the most brutal scenes in The Expendables 2, the slaughter of Villains assassins in the old army base and the killing of Villain, are both staged to mimic the classical western scenes of the 50s which is funny since it is a clear self-ironic reference to the perceived outdatedness of these men. Similarly amusing is watching Trench and Mr Church use a SMART, a modern but tiny car, which is clearly not made for their body frame, to chase the bad guys. 
These humorous elements in combination with the excess of brutality, destruction and violence in The Expendables trilogy allows audiences to accept what is today normally unacceptable: the use of violence and brutality to establish male dominance and superiority by men whose age, social background and ultra-masculinity opposes current views of socioculturally acceptable constructions of masculinity (Connell, 2005; Connell, 2014). After all, if the protagonists themselves do not seem to take their own behaviour and claim for reinstating an archaic masculinity to save a world where not many things are going right really seriously (Jeffords, 1994), then why should audiences? As such, in The Expendables trilogy humour is instrumentalised to take the sting out of both the harsh ideological criticism and the presentation of an alternative and highly controversial masculinity ideal. This strategy is undoubtedly aimed at increasing the potential target audience and thereby the commercial success of the films despite their quite reactionary narrative construction. This is a quite popular but nevertheless genius manoeuvre applied by many media products of present times (Tasker, 1993b).

Mass media are substantially dependent on understanding and prematurely recognising new trends, and they have undoubtedly managed to successfully adapt their products to today's Zeitgeist of resistance based on the concept of ptolemization. According to Žižek, ptolemization occurs "[W]hen a discipline is in crisis, attempts are made to change or supplement its thesis within the terms of its basic framework" (Žižek, 2008, p. vii). Although Žižek refers to a crisis in academic disciplines, I believe the concept of ptolemization can also be applied to socio-political systems in crisis, powerful socio-cultural agents reproducing and subsequently maintaining this system and to individual negotiations of identity within the frame of the respective ideology. Ptolemization is hence a crucial concept not only for the analysis of The Expendables but also for the exploration of individual identity constructions of older German men which follows.

Recently the flaws of neoliberalism and its institutions - the state, the church, and the law have increasingly come to light and have led to a hegemonic position of the post-modern individual characterised by critical distance and resistance to the ideological system. One seems to question authority and outdated stereotypes continuously and to refuse indoctrination (Žižek, 1992, 2006, 2008). That this resistance does not translate into a discussion of alternative socio-political approaches (Bingham, 1994; Hall, 2000, 2015; Hall \& Winlow, 2015), is, to my mind, proof for the successful ptolemization of the current ideology and related values and principles. In other words, most people are more than aware of the fact that neoliberalism has significant weaknesses and is inherently unjust and therefore, ultimately self-destructive. Nevertheless, since neoliberal system individuals live in seem to be part of a natural order and, as a result, changes to it are next to unthinkable, it 
appears impossible to move beyond fighting for the interests of marginalised groups and their rights within the boundaries of neoliberal society.

Within this context, many products of popular culture seem to break conventional narrative patterns and to question hegemonic narratives linked to gender, race, social class, or ageing, to name but a few, to satisfy the audiences' attitude of resistance which calls for specific patterns of neoliberalism to be criticised and changed. However, the alterations in the narratives are rather superficial and continue to perpetuate key neoliberal values such as consumerism, productivity and autonomy. When viewed through the lens of ptolemization, The Expendables trilogy is hence by no means a rebellious piece of filmic work which calls old and young to arms to fight against neoliberal views of ageing and masculinity.

There can be no doubt that all three films are by and large commercial products aimed at making the highest possible profit. They are a result of being produced in Hollywood for the mass market and thus follow conventional, easily understandable and sellable cultural narratives of ageing and masculinity. Successful ageing in The Expendables series is hence defined as an individual and controllable fight against ageing aimed at staying fit, selfreliant, productive and, above all, youthful as long as possible. Successful ageing is in all films skilfully interwoven and contrasted with hegemonic narratives linking ageing to decline since the older age of the main cast cannot be ignored entirely and more importantly, since their victorious and manly fight against ageing then becomes even more pronounced and admirable.

The films are hence far from facilitating a more differentiated understanding of ageing and gender but they, on the contrary, reproduce and support a view in which a masculinity ideal closely tied to controlling oneself, and everything and everybody else by blatant brutality and aggression is constructed as the basis for overcoming marginalisation due to age, social background or gender. Hence the films communicate a narrative which is deeply rooted in the neoliberal value system and which could be summarised as follows: The 'disabilities' of being a woman and/or of being old cannot be eliminated, but they can be significantly reduced and partly overwritten provided one follows the neoliberal dictate of staying youthful, productive, and, most importantly, self-reliant and in control by consuming products which support and enable youthful looks and behaviour and individual autonomy. As such, The Expendables trilogy is not only an illuminating example for the ptolemization of dominant neoliberal master narratives but it is also exemplary for the socio-cultural frames available for the identity construction of older men in neoliberal societies. An exploration of the question of how German men use precisely these socio-cultural master narratives to construct their identity in older age will be in the centre of the subsequent interview analyses. 


\section{Reflexive Comments on Interviewing Process \& Analysis Reflections of Retired German Men}

\subsection{General Realisations, Researcher's Position \& Initial Development of Interview Questions}

Since my research is mainly concerned with the investigation of empirically invisible processes, it quickly became clear to me that to gain a better understanding of how retired German men handle older age and retirement and how these aspects have impacted their identity construction and life-course narratives the most suitable research method would be the conduction of interviews. I believed interviews to be the most effective research approach to allow some access to the lifeworlds of my participants (Haraway, 1988) but I also considered a discourse-based method to best fit my own abilities as a researcher (Patton, 2014). Having been a teacher and lecturer for the past nearly twenty years a large part of my professional life was and is dependent on the ability to create an environment in which students feel that they can express their thoughts freely, which in turn then enables me to elicit responses and further critical reflection (Argyris, 2002).

Essential elements for creating an atmosphere in which all participants in communicative exchanges feel comfortable to articulate openly what is on their mind are, in my view, a genuine interest in what others have to say and an attitude which is not based on preconceived ideas which one only seeks to confirm in the communication with others (Harding, 1992). In addition, in my experience the development of mutual trust in discursive practices is substantially facilitated when the communication is not one-sided but when I allow the counterparts in any respective discussion access to my own thoughts, opinions and experiences. This allows for communication on eye-level rather than top-down, and this further supports trust and subsequently deeper insights into the life-course narratives of others (Burr, 2006; Denzin, 2001). However, the quid-quo-pro approach can, if one is like me and rather talkative, quickly and often unintentionally become imbalanced and veer towards a self-centred monologue of the person asking the questions rather than of the person or group of people one was interested in finding out more about (Patton, 2014).

This is particularly true within the context of conducting interviews since their aim and the relationship to the interviewees are entirely different from informal and often less structured ways which characterise conversations in a professional or private context. Indeed, the process involved in the development of the interview questions, the execution of the interviews and the analysis of the interview results has, despite my experiences in instigating student participation, been quite challenging for me (Patton, 2014). 
As discussed in chapter 2 of this thesis, the strong emphasis on objectivity and rationality within the German research context, particularly in management science, necessitates the researcher to follow strict procedures to ensure validity and reliability of the investigation (Chang \& Aldred, 2014; Koivisto, Thomas, \& Aslama, 2008; Kuhn, 1970). These are the prerequisites for ensuring the credibility of one's research and for subsequently gaining a reputation as a scholar. To enable 'objective' research crucial aspects are not only that the researcher must stay distant and non-emphatic observers when interviewing but also the assumption that the participants will often not be willing to disclose voluntarily the information one is seeking for. In Germany, there is a common view that participants in interviews are consciously or sub-consciously untruthful due to the desire to please the interviewer, to fulfil social expectations and/or to maintain a consistent and positive selfconcept - an aspect many of my German students mention instantaneously when asked for the challenges involved in using interviewing as a research method. As a result, essentialist approaches towards interviewing in Germany are frequently characterised by not fully disclosing the research aims not to taint responses and apparently increase objectivity (Baur \& Blasius, 2014; Witt \& Redding, 2009).

It did not overly worry me that my interviewees could deceive me willingly or unwillingly for above-mentioned reasons or so I thought. In fact, one could say that it were precisely these aspects which interested me since I firmly believe that everyone's life-course narratives are in large part the result of the wish to construct a harmonious identity that is compatible with accepted socio-cultural and personal standards (McAdams \& McLean, 2013). This permits the individual to receive the kind of interpersonal recognition most people, at least in the occidental world, so desperately need to sustain a positive selfperception (McDonald et al., 2017).

Notwithstanding this, a leopard cannot easily change its spots, and there is hence a great danger that, without you noticing, certain elements perceived as natural in your culture sneak in even though they are highly inappropriate for the research realised. My embeddedness in the German culture and its preference for control and order in the research process is with hindsight clearly evident in the choices I made in my empirical investigation (Cramer, 2015; Schommer, 1990; Zimmer \& Toepler, 1996). I decided to base my exploration of the lifecourse narratives of older German men on semi-structured interviews since I did not feel experienced and subsequently confident enough to dare using an unstructured interview approach only loosely based on pre-determined thematic units but mostly developed through probing (Creswell, 2009). Although I believe, that this choice is quite typical for anyone who is a novice in scientific research and interviewing as I am (Baxter \& Jack, 2008; Turner III, 2010), when looking back it is also clear to me that was not the only reason for selecting a 
semi-structured approach to interviewing. My choice was surely also an outcome of my cultural background and the need to feel more control over the research process.

At the same time, and this is yet again clear proof of my ambiguous socio-cultural situatedness, it was crucial to me to take a position which supported an interview situation in which both, the interviewer and the interviewee are equal participants in the research process. This is rather untypical for the German research context since the essentialist research paradigm demands of the researcher to stay objective and non-empathetic to ensure objectivity (Koivisto et al., 2008). This position often leads to an unbalanced hierarchical relationship in which the researcher somehow from above indifferently investigates the subject of interest and in which the researcher, and only the researcher, decides which information to disclose to the interviewee and which is withheld (Haraway, 1988). However, I share the understanding that objectivity in the research process is impossible since " [T]he thought of an age is of an age, and the delusion that one's thought can escape historical locatedness is just one of the thoughts that is typical of dominant groups in these and other ages" (Harding, 1992, p. 62). In other words, the socio-cultural influences human beings grow up with and in are impossible to discard since they are not only an essential part of the individual's identity but also often mostly sub-conscious. As a result, taking on an objective position in research such as this is, in my view, neither possible nor, and this is particularly true within my research context, desirable .

Since my main interest in the interviews I conducted lay in gaining access to the experiences and emotions of my participants, it was, thus, of utter importance to quickly establish an interview situation based on trust and mutual respect resembling an informal conversation between acquaintances which allowed the participants to, as openly and as uninhibitedly as possible, speak their mind (Mason, 2017; Patton, 2014). To clarify that the interview was based on genuine interest and not on attempting to tease out specific answers I wished to hear, I emphasised in the introductory remarks prior to the first interview question that there was not such a thing as 'correct' or 'incorrect' answers while stressing that I was interested in gaining a better understanding of their experiences with the transition from employment to retirement.

Being a woman, the age gap of thirty years between me and the participants and related higher level of professional and personal experiences on the interviewees' part as well as the fact that all my interviewees used to work in high positions in a corporate environment whilst I was a lecturer of so-called soft skills, a profession affiliated predominately with women, placed me in an anything but superior position to my interviewees. In addition, I had encountered all interviewees in social gatherings before even if, I had, apart from my father, never had lengthy or in-depth conversations with them. These aspects as well as the fact that 
all participants have children my age, supported the establishment of a relaxed and cooperative interview situation in which all participants displayed a high level of willingness to allow me access to their life-worlds (Blichfeldt \& Heldbjerg, 2011; Robertson \& Hale, 2011).

Nevertheless, because my participants did not have experiences with research interviews and therefore did not know exactly what was coming their way, in the very beginning of the interviews some level of tension and nervousness was visible in all my participants. This is not surprising given the novelty and resulting uncontrollability of the situation in a sociocultural context in which control is held in the highest esteem and in which information is an extremely highly valued currency (Bourdieu, December 1998; Harvey, 2007b). In addition, my own inexperience, the high significance of this research stage and the fact that, except for my Dad, I hardly knew the respondents, increased my own insecurity and slight uneasiness. How far this showed I cannot tell, but surely it had some influence on the overall interview situation (Baxter \& Jack, 2008). As a result of my experiences in teaching in a one-to-one situation as well as of the research I had done to help me develop the interview procedure and questions, I decided for three main strategies to establish and strengthen the rapport and trust between my participants and me which, to my mind, was the basis for accomplishing sufficient depth in the interviews (Argyris, 2002, 2008).

First of all, I believed that it was essential to provide the participants with some control of the situation by giving them a choice where the interview would take place. Three of the interviewees chose to invite me to their house (Burkhard Rosenfeld, Prof. Dr Günther Schotten and Dr Lars Bühring) and one (GB) I interviewed at my own place. My supervisors had warned me beforehand that conducting interviews in a private surrounding could be risky since I would not have the possibility to protect myself against verbal or physical assaults. However, I decided to go against the advice of my supervisors as, in my view, the risk was rather small given that my respondents were known to my family and me and as the benefit of establishing some trust and rapport before the interview had even started outweighed the risks. Moreover, visiting the interviewees at their home provided me with some additional insights into my participants' lives. Not only was I able to see where and how they lived which somehow added depth to their life-course narratives, but I also had the opportunity to briefly meet their wives which, despite the fact that they were not part of my investigation, I found valuable. It aided me in better understanding what my participants had told me about their partnerships.

Secondly, to generate data as rich as possible, I knew that I had to force myself to let the interview develop freely and spontaneously. That meant that probing and following up on what my participants said was crucial whilst asking each and every question I had formulated 
in the order they were written down was not (Flick, 2009). Being a novice in conducting research interviews and being a German who is very dependent on retaining a feeling of control, I found this realisation quite daunting even though this was an approach familiar to me from one-to-one tuitions in English language teaching (Hughes, 2005; Merriam, 2007). Nonetheless, when teaching English, it was irrelevant where the conversation ended as long as my students spoke English, but this was obviously not the case in my research interviews. I needed to find a way to simultaneously permit my respondents to lead the interview whilst ensuring that the topics I was interested in were covered in as much depth as possible. Multitasking has never really been a key competence of mine, and therefore I decided to develop a concept map covering the main areas which had transpired during the previous steps of my project (see Appendix D) in addition to the questions I devised. This served me as a kind of safety net as it allowed me to quickly check during the interview which topics were already covered and which were not. This strategy turned out to be effective and the more so, the more interviews I had conducted as I consequently became both increasingly familiar with the questions and developed more confidence in my interviewing capabilities (Mason, 2017). Lastly and most challengingly, to gain deeper insights into my participants' views on constructions of masculinity and ageing, their own and others, it was not only essential to transmit to them that everything they told me was of high significance to me, but it was also, and more importantly, of highest relevance to emphasise that whatever they told me was received by me as neutrally and non-judgementally as possible. To show empathy and to express understanding of their viewpoints as well as the choices they had made was, in my view, most critical to encourage my participants in sharing their beliefs, experiences and emotions with me. Patton calls this research position 'empathic neutrality' (2014, pp. 56-60) and whilst showing empathy came quite naturally to me, neutrality was much more difficult to achieve. Eliciting as much depth as possible in the input I received by asking follow up questions without leading my participants in a particular direction which fit the research I had done prior to the interviews, required constant alertness and reflection (Morley, 2008; Stewart \& Sambrook, 2008).

I believe that all in all, I managed to stay neutral, to keep an open mind by attempting not to let my own feminist views on the construction of identity in older male age and on gender hierarchies influence the interview questions, and particularly the follow-up questions and their analysis. Nonetheless, retaining neutrality was an ongoing challenge throughout the whole process involved in the empirical stage of this thesis. In any case, I have sincerely tried to credit the trust my interviewees showed to me by staying as impartial, receptive and reflexive as possible and I hope that my respondents will feel that they have been adequately represented in my analysis. 
Within this context, to formulate questions that were both open and non-leading was imperative to minimize researcher bias and maximise the richness of data gained from my participants (Merriam, 2009). The initial interview questions were based on these considerations and an outcome of my literature review of key texts in ageing and gender respectively men's studies as well as of the initial film analysis of The Expendables trilogy. During the theoretical exploration of the socio-cultural construction of ageing and particularly male ageing and conceptualisations of masculinity in the occidental world, certain aspects recurred. Key themes related to ageing were in particular ageing viewed as decline characterised by an increasing loss of mental and physical abilities, illness, financial instability, social isolation and a potentially resulting state of depression whilst the concept of successful ageing is conceptualised as linked to continued mental and physical fitness and health, autonomy and productivity (Baars, 2013; Fung, 2013; Gullette Morganroth, 2004; Laceulle \& Baars, 2014; Segal, 2014). Although these hegemonic narratives apply to both men and women, their socio-cultural interpretation is gender-specific and as such these narratives contribute to the continuation of gender hierarchies into older age (Dolan, 2018; Dolan \& Tincknell, 2012).

In the literature, constructions of masculinity were viewed as hallmarked by their hierarchical and context-bound division in hegemonic, complicit, subordinate and marginalised masculinities. Hegemonic masculinity, my research focus, was described in the literature as predominately linked to being white, heterosexual and as having access to much cultural and economic capital expressed through a high educational level and a lucrative and influential position often in the world of multinational corporations (Cheng, 1999; Connell, 1998; Connell, 2005). Another element critical in perceiving oneself as being part of the hegemonic group of men and underlying before-mentioned characteristics appeared to be emotional and physical control of oneself and others and linked personal autonomy (Coston \& Kimmel, 2012; Kimmel, 2012). However, prior understandings which saw masculinity particularly evident in the display of bodily strengths and superiority and in dominating and subordinating others by using physical violence, are nowadays supposedly outdated and representative for marginalised masculinity constructions (Hall, 2002).

In the first approach towards my interview guide, I chose to start with asking my participants to describe the progression of their career to me since I believed this to be a good starting point to break the ice and get my participants talking and since I was particularly interested in how significant their professional life was for their self-perception. Probing and the follow-up questions were aimed at gaining more insight into the aspects which the interviewees particularly liked and disliked in their jobs so that I was better able to understand if aspects such as control, autonomy, status and dominance played a role in the 
uttered likes and dislikes. The next section focussed on the significance and structure of their personal life during their employment. This helped me generate more depth with regard to my participants' priority settings during their working life. After that, I moved to the topic of retirement and all questions in this part centred on how retirement had impacted their private life, their status, their emotional and physical condition and, most importantly their self-perception (see Appendix B).

Subsequently, I discussed how they experienced the process of ageing with my participants. Within this context, I was particularly interested in exploring how my participants' identity construction had changed with increasing age, in how they dealt with age-related mental and physical changes, how they handled potential fears and worries linked to the ageing process and what they felt to be positive about older age. In the next section, I asked the interviewees about their financial situation so as to better understand how, if, at all, the financial situation of my participants affected their life in older age. In the next part of the interviews, I enquired about the relationship of the interviewees to younger people since I wished to explore the possibility of intergenerational cooperation.

In the next section of the interview guide, I aimed at eliciting my respondents' views on socio-cultural attitudes towards ageing and older age in the West, media representation of ageing and on how they evaluated these and their impact on their identity construction in older age. Lastly, I enquired about some demographic details such as what nationality my participants were, if my participants had ever lived abroad or if they lived in their own property. These questions were aimed at providing some more detail about the socio-cultural background of the interviewees (see Appendix B).

Although the general structure of the interview proved overall to be useful and I therefore only marginally adapted it from one interview to the next, as a result of my post-interviewing reflections, I changed some questions, removed others and also added some questions which were not part of the original set of questions. These alterations and their rationale will be discussed in the next section.

\subsection{Reflections on Interviewing Process ${ }^{2}$}

Despite the fact that I was aware of my cultural embeddedness and resulting hang-ups, I nevertheless shied away from asking explicit questions tackling the issues I was interested in particular with regard to masculinity. In fact, during the pilot interview, I discovered that I had not asked one direct question about the participant's own understanding and construction of masculinity and any changes related to older age in their conception of

\footnotetext{
${ }^{2}$ This section is predominantely based on my notes on the interviewing process - see Appendix E
} 
masculinity. Before I conducted the pilot interview, I was, and this is in my view of crucial importance, not in the slightest aware of this forbearance. This subconscious disregard for such a crucial aspect in my research is I believe clear proof for my cultural blinkers (Schommer, 1990; Schön, 1983).

Of course, I intended to find out about my interviewees' views of masculinity, but instead of asking directly, I decided to rather circle around the issue without ever mentioning the word masculinity explicitly. This approach resembles the above-discussed German approach towards qualitative interviewing based on deceiving the interview participants to gain insights assuming that the participants would not willingly and openly disclose these if asked explicitly. This realisation was a significant learning experience for me since it aided me further in critically reflecting on my own socio-cultural embeddedness and in moving beyond it as far as possible (Argyris, 2002; Guillemin \& Gillam, 2004). I subsequently adapted my questions to include direct questions about masculinity directed at gaining a better understanding of my interviewees' perception of masculinity and femininity and the effect of ageing on these perceptions whilst generally reviewing all questions and adapting some questions so that they were phrased as directly and transparently as possible.

In addition, I noticed that although sexuality in older age is, without a doubt, of high significance for my research context (Calasanti \& King, 2005; Dolan, 2018), tackling this issue was extremely difficult for me. Although I had, after having discussed this issue with my supervisors, included questions on intimacy in my pilot interview, I could, in the end, not bring myself to ask these questions since I did not feel comfortable asking such private questions. Surely this was also based on socio-cultural influence, but in this case, at least in my view, it was less a result of being affected by the German research standards and procedures but more of my upbringing. This has taught me to show respect to older people which included not unduly intruding on their privacy which I felt would be the case when asking them about their sexual activity. At the same time, my own age and sex created a distance between my male and older participants and me. In other words, the age gap of thirty years and the different sex had a substantial impact on what I believed I could ask without risking to provoke an uncomfortable atmosphere and subsequently losing rapport with my participants (Patton, 2014).

I was and am aware of the fact that my interviewees might have had no problem with answering questions about their sexual life, but in my view, the chances for this reaction were rather slim. First of all, their generation is, in my experience, much less open towards publicly debating their sex life than following generations (Wells \& Twenge, 2005). Moreover, my relationship with the participants did not really permit revealing such personal details because I knew them too little or, in the case of my father, our relationship as father 
and daughter made it somewhat problematic. My experience in teaching has also led me to the realisation that if I felt uncomfortable asking a specific type of question, this would affect the insight gained (Mason, 2017). I then posed questions simply to tick them of an imaginary or, in the interviews, a real list and quickly move on to the next questions without probing for more information. In the end, I decided not to include any questions on the sexual activities of my participants since I did not believe that the risk could outbalance the insight generated.

During the pilot interview, I also recognised that the questions with a thematic focus on the media representation of older age were based mainly on assuming that everybody has similar viewing habits with regards to media as I do (Argyris, 2002; Bolin \& Skogerbø, 2013). I thus asked if my respondent felt that the media representation mirrored his own lived reality. This question led to a little bit of confusion since the participant did not watch TV as much as I did, avoided watching advertisements and when going to the cinema did not select films with older protagonists. As a result, he could not recount any media representations of older people. Hence this part became hypothetical, and the respondent seemed to feel as uncomfortable as if he had forgotten an essential answer in an exam. In hindsight, I realised that most people do neither watch as much nor the same content on TV as me. I, therefore, tried to adapt this section of my interview so that it became less vague and hypothetical. I believed that there needed to be more input from my side and I decided to use some visuals showing older people I had found online to then ask the interviewees which pictures they felt represented by and why (see Appendix C).

Shockingly I also became aware that I had not asked one specific question about cultural differences in the understanding of ageing, although this was a significant research interest of mine. Why I had not done this is unexplainable to me, but I believe it was a result of my inexperience (Turner III, 2010) and the pressure I felt to not ask too many questions to not over exhaust my respondents (Russell, 1999) and to support the manageability of my data (Merriam, 2009). In addition, I had initially thought that I could elicit some more detail during the last part of my interview in which I asked some demographic questions including if my participants had been to other countries or even lived in another part of the world. Nonetheless, after the pilot interview, I felt that I needed to include some specific questions before the last section with a focus on socio-cultural variances in viewing age and ageing. Lastly, I noticed that I needed to include some more questions about the religious inclination of my participants since it seemed logical to me that how you view ageing and linked finiteness of being is majorly influenced by what one believes to happen after death which in turn could have an impact on how you view and handle your own ageing. 
Taking part in the Women, Ageing and Media (WAM) summer school in June 2017 markedly aided me in reflecting on my interview questions and structure. As a consequence, I recognised that even though I had assumed that my research focus would ensure my own sensitivity towards ageist views, I was not immune against over-simplified categorisations of older people. In fact, I had not asked one question about future plans of my participants since I had assumed that due to their older age they would focus on the past and the present rather than on the future. This was a crucial, although shocking, realisation since it illustrated my own socio-cultural limitations and alerted me to the necessity of closer questioning my own pre-conceived, often sub-conscious understandings of ageing (Ayalon \& Tesch-Römer, 2018; Kinsella, 2007; Nelson, 2005).

Since much of the input received during the summer school dealt with how older people handled technological advances, another outcome of my participation was that I extended the area of the interview which had a focus on their relationship to younger generations by including two questions enquiring about what areas the participants felt to be in an advantageous respectively disadvantageous position towards younger people. These questions were aimed at gaining a better understanding of how the interviewees evaluate themselves, their capabilities and their mental and physical performance in comparison to younger people. Within this context, I was particularly interested in whether, on the one hand, aspects such as technological advances and their use by older people would be mentioned and, on the other hand, whether concepts such as wisdom, life experience and serenity, often linked to older age, would be brought up by the participants (Cruikshank, 2013; Gullette Morganroth, 2004; Jennings, 2015).

My realisations from the pilot interviewing until conducting the first interview led to a high increase in the questions from forty-four to fifty-eight. During the first interview, I then, not surprisingly, felt that there were too many questions since answering this amount of questions was strenuous for the participants and for me, particularly when considering the need for probing follow up questions as well. I thus decided to reduce the number of questions by excluding some, by summarising others, by generally being more direct and by following a more flexible approach based on the thematic units developed in the previously mentioned mind-map. This strategy, I believed, would allow me to gain more depth by following my participants in their line of thought more flexibly which would, in turn, lead to a more intense reflection on the topics that interested me. I also felt that I needed to take myself back more since during the first interview, and as a result of my desire to quickly establish rapport with Burkhard Rosenfeld, there was sometimes too much input from my side. I therefore aimed at finding a better balance between my reactions and what my respondents said in the next interview (Patton, 2014). 
Tackling the issue of media representations of ageing and gender, was, despite providing visual input contextualising notions of ageing in ads, still highly unsatisfactory. Instead of leading to a discussion about the public images of ageing, the participant simply chose the pictures representing him best. Since most people wish to support a positive and stable selfperception which is, however mostly dependent on how one is perceived by others, it was not really surprising that Burkard Rosenfeld chose the photos clearly based on notions of successful and active ageing (McAdams \& McLean, 2013). Although insightful, this was nevertheless not the intention of the visual input, which, after all, I felt was too leading and manipulative. After all, who would select to view him- or herself as frail, as controlled by others and as characterised by declining physical and mental abilities if there is an alternative? Thus, I decided to remove the visuals in the next interview and try another approach. I changed the order of questions and first asked about attitude to ageing in society and then inquired where my participants thought these constructions of ageing were based on. I hoped that this structure would automatically lead to a discussion of media as a crucial agent of socio-cultural standards and values. In addition, I believed this alteration in the structure and content of the interview could elicit relevant comments on other socio-cultural agents such as the law, educational institutions or politics (Bauer, Heinemann, \& Lemke, 2017).

Lastly, during my first interview I realised that in addition to asking about my participants' definition of masculinity, which, I thought, provided particularly insightful comments besides being an effective lead-in, it could increase the depth of responses if this question was followed up by asking if my participants believed their understanding of masculinity to be the result of their upbringing and childhood experiences (Bourdieu, 1990, December 1998; Fausto-Sterling, 2000). This alteration turned out to be a useful addition not only because it somewhat followed naturally but also as it supported the richness of the information received and focused my participants' on reflecting on the past. Subsequently, this supported a smooth transition to the next part of the interview in which I asked about their career development.

Although the inclusion of a question on the influence of upbringing on the perception of masculinity worked well, I decided against a specific question about the army service, which I had considered, since I believed it would most likely come up in their reflection on their professional development, and since I did not want to extend the number of questions further. Additionally, I excluded the last part of the interview in which I intended to gain some information about the demographic background of my participants as I had realised that these facts would be covered during the interview without having to ask about it specifically. 
The short time span of one day between the first and the second interview proved to be disadvantageous although in this particular case unavoidable as the participant had made several travel arrangements for the coming months which would have made finding another time to meet very difficult. And, indeed having just one day to act on the realisation from the first interview was challenging but possible. As already mentioned above, I managed to reduce the number of questions from fifty-eight to thirty-six, mainly by merging several singular questions about one specific theme into one or two more general question(s). The adaptation of the interview from a more structured and controllable to a more open and flexible approach emerged as particularly useful on several levels (see Appendix B, Interview 1).

First of all, it increased the flow of the conversation and resultantly the richness of the data since the participants were entirely free in how they interpreted the questions without being limited and led by specific questions (Mason, 2017). Moreover, and related to the previous, the openness of the questions allowed for developments of the conversation I had not expected, which therefore proved to be particularly interesting and insightful. This also aided me in not saying too much during the interview since, besides listening and probing, I also had to ensure that I had covered all relevant themes and not to let the conversation veer off the topic entirely. This forced me to rely more on the thematic units defined in the conceptmap and on probing for more detail if need be rather than on the ticking off of questions (Ritchie, Lewis, Nicholls, \& Ormston, 2013). This had from the start been my aim and, I believe, that, in general, it worked quite well. Nevertheless, I felt that I needed more practice since, as mentioned above, multi-tasking is not an area I excel in which means that I was continuously worried about having forgotten to tackle a particularly important question. This somehow stressed me which in turn could have impacted the interview situation negatively since it could have led to me to be more interested in covering all pre-determined areas of interest than in what my interviewees had to say and in probing for more detail (Patton, 2014).

That this was, in my view, not the case, was less a result of my improving abilities as a research interviewer but more of the fact Prof. Dr Schotten, my second interviewee, was much less talkative and more to the point and precise than Burkard Rosenfeld, my first interviewee, which could be the result of his profession as a notary as well as being a character trait. However, I believe that despite the fact the interview took a shorter time to conduct and the answers were not quite as extensive as in my first interview, it was by no means less informative than my first interview. In addition, the precision and goalorientation of Prof. Dr Schotten's answers aided me immensely in probing and keeping in 
control of the interview despite its more flexible structure (Rosenfeld, Personal Communication, 17.07.2017 \& Schotten, Personal Communication, 18.07.2019).

Not using the visual input and asking some more general questions about the representation of ageing and older people led quite naturally to a discussion of the representation of older people in the media but also, as I had hoped, to a more general reflection on the basis of socio-cultural narratives of ageing and masculinity. This nearly automatically provoked a closer focus on cultural differences in the conception of ageing and masculinity. I was quite happy with this particular change in the question formulation and structure since I felt I had finally found an effective way of approaching this thematic focus (see Appendix B, interview 2).

Interestingly I noticed that I felt somehow more intimidated by Prof. Dr Schotten than by Burkard Rosenfeld. This entirely subjective feeling, which was not in the slightest based on the way Prof. Dr Schotten acted towards me or how he answered my questions, was possibly the result of me being well aware of my participant's titles and his academic work. In hindsight, I think that I felt that Prof. Dr Schotten was more in a position to evaluate me and my work. It was quite a shocking revelation that apparently I could not as easily, as I had thought, free myself from public perceptions which link academic titles to intellectual and sometimes even moral superiority (Bourdieu, 2011, 2018). I had, however, known for a long time that especially situations in which I am formally or informally assessed, I experience the pressure as unsettling. This makes me feel overly insecure, which then frequently results in an unsatisfactory performance below the standard I am capable of. Nonetheless, within the specific context of my research interviews, I believe that my insecurity did not negatively influence the interaction between Prof. Dr Schotten and me. Even if the rapport between him and me might have been a little weaker than with my previous interviewee, I believe that my insecurity and the resulting uneven power relations increased my interviewees' confidence and subsequently his trust in me and his willingness to talk openly about his experiences and opinions (Mason, 2017). This perception was later confirmed when Prof. Dr Schotten told my father and me on different occasions that he was surprised at how much he had actually told me (Schotten, Personal Communications with Lisa and Lars Bühring, n.d.)

After the second interview, I decided to exclude specific questions about the financial situation of my interviewees. I had noticed in both interviews that asking about the economic status of the participants made them and me feel quite uncomfortable. This was probably due to our socio-cultural background in which talking about money is considered a sign of bad manners and lower social class since it is considered as inappropriate boasting indicating low self-esteem and a desperate need for social recognition. This is particularly true for the generation my participants belong to since they grew up in a time when the money taboo 
was much stricter than it is today (Alsemgeest, 2014; Needleman, 1994; Trachtman, 1999). To not endanger the rapport I had developed with my participants, I only touched on the topic in the first interviews without asking for precise numbers. I also realised that the issue of money and financial stability would be broached naturally in the course of the interview although mostly indirectly when talking about the lifestyle of the interviewees and thus decided that there is no need to ask about money matters explicitly (see Appendix B, Interview 2).

There was a period of five months between the second and the third interview. The more extended period of time between the second and third interview had not explicitly been planned but was the result of my realisation that I needed more time between one interview and the next to act on the outcome of the interview reflections, the tight schedule of my third interviewee and my wish to start transcribing the first two interviews. All in all, I believe the long time between the second and third interview was beneficial (Ritchie et al., 2013).

The transcription process, as well as the continuous work on the literature review, enabled me to reflect on the relevance of questions asked and on the effectiveness of my probing. Overall, I was satisfied with the questions, but when reviewing the literature, I recognised that I needed some focus on chronological time and subjective perceptions of age (Baars, 2013, 2015). So, I added two questions with a specific focus on how my participants define older age and how old they felt. In my view, this would lead to a discussion of the concept of chronological time and its fit with the subjectively felt age. Besides this, and the exclusion of the three questions about the financial situation of my participants, the structure and questions generally remained the same as in interview two even though in the question 'Has your position as retiree had an influence on how you view yourself?' I added '... as a man?' since I thought this addition would be better suited to capturing the particular perspectives of my male participants (see Appendix B, Interview 3).

There was an excellent rapport between the third interviewee and me, possibly since GB is an outgoing, reflective and very open person. However, this also meant that the conversation veered off focus frequently, and I was forced to approach the interview much more flexibly than in the previous ones. Although I found it rather stressful to mostly base the interview on thematic units rather than on proceeding from one interview question to the next, it worked astonishingly well, which increased my confidence in my interviewing abilities. In general, the interview with GB had provided me with an, up to this stage, unprecedented level of richness. It not only allowed me deep insights into my participant's life but it was also unique in revealing experiences, feelings and reflections that were in many ways contrary to the interviews I had conducted before (GB, Personal Communication 15.12.2017). In fact, I had shortly considered to not conduct any more interviews for the 
before-mentioned reasons but after some reflection decided on doing one more interview with my father.

That I felt more satisfied with the process and outcome of the interview with GB was possibly, apart from the participant and me being on the same wavelength, also the result of gaining more experience in research interviewing. Indeed, in the period between the second and the third interview, I had conducted another interview which, after serious consideration, I excluded. The interview with Erich Schüle, a retired high school teacher of sports, was rather short and characterised by his reluctance to talk and reflect about older age and masculinity. It was also the only interview in which the participant continuously asked me, probably because he sensed my increasing frustration with his brief and little informative answers, what I wanted to hear. The differences between the first two and the third interview probed me to reflect on the reasons for this and led me to the realisation that besides possibly being the result of Erich Schüle's personality, it might have also been since the participant did predominantly not match the hegemonic masculinity ideal. This put him in a more defensive position and additionally meant that he could not relate as well as my other participants to the questions I asked (Connell, 2005; Coston \& Kimmel, 2012).

This experience aided me immensely in my research as it forced me to think about the aim of my interviews and the selection of the participants intensely. I had initially included the participant precisely because he did not match current hegemonic masculinity as perfectly as my other interviewees. I believed including him would increase the depth of my research by exploring a somewhat different perspective on ageing and masculinity. However, after the interview, I realised that incorporating his perspective would be like opening Pandora's box. In other words, I thought that including the life-course narrative of someone whose socio-cultural background, former profession and self-perception firmly link him to constructions of complicit, in parts perhaps even subordinate, masculinities would go beyond the aims of my research and thus compromise its depth and logical development. In my view, the interview with Erich Schüle, my reflections on it and the decision to exclude it, therefore supported a more consistent and focused research approach (Mason, 2017; Merriam, 2009). Although during the pilot interview I felt that being too close to the interviewee and knowing him too well can be a problem (see Appendix E), after thinking about it intensely, I decided to, after all, conduct the last interview with my father for several reasons. First of all, observing my parents' very different approaches towards managing the ageing process has, besides my own experiences with getting older, been the main reason for my research focus and as such it seemed logical to me to include, at least, my Dad in the research. I really wanted to find out more about his life-course narrative(s), his identity construction and any changes to this identity construction in older age. Moreover, my father has taken a vital 
interest in my research, and I thus found it appropriate to allow him to share his perspective on ageing with me. Furthermore, my father used to fit the hegemonic masculinity ideal in many ways, and I believed he could provide crucial and relevant insights into the identity construction of German men who used to be part of the hegemonic class of men in older age. Finally, including my father in the interviews I thought could be a beneficial corrective since our closeness would motivate me additionally in exploring his and the other interviewees' lifeworld and life course narratives sensitively so that he and the other participants would feel appropriately represented by my account of what they had said (Harding, 1992; Mason, 2017).

Since there was a time gap between the last and this interview and I had written up my literature review in the meantime, I included specific questions on the successful ageing paradigm which before I had instead, in the German manner, circled around than explicitly asked about (Segal, 2013, 2014). These new questions turned out to provide exciting insights since my father defined the paradigm of successful ageing somewhat differently to the aspects mentioned in the literature (compare chapter 7.4). I also decided to approach the questions regarding the media representation slightly differently and take a less explicit approach by rather asking about where my respondent thought the socio-cultural understanding of ageing comes from. This was intended to allow for more flexibility in the answer, which worked well but did not yield markedly different input from the interviews before (see Appendix B, Interview 4).

My father was very focused and precise in his answers which is reflected in the relatively short time the interview took and also in the clarity both in speech and content of my father's answers. This was potentially a result of both our many previous discussions on the topics covered and his wish to provide me with valuable input and his somewhat rational and controlled personality (Bühring, Personal Communication, 31.07.2018). Nonetheless, I believe that his answers reflect his views and are not aimed at pleasing me since I had repeatedly discussed with my father that the aim of my research is not to confirm preconceived ideas of mine but to gain a better understanding of the lives of my older male participants. Although it somehow sometimes felt a bit awkward to be in a situation in which we covered topics we had discussed before in a much less structured and informal way, overall the interview went well, and I believe it was a fruitful and enriching experience for both of us. I am therefore happy with my decision to include my Dad's perspectives on male ageing despite my former worries. 


\subsection{Reflections on Interview Analysis}

In many ways, the analysis of the in-depth interviews I conducted marked the climax of my research journey. However, there were also numerous challenges involved in this stage of my research. Not being able to touch type negatively impacted the transcription process since it slowed me down immensely. After the first transcription, which took me weeks, I decided to have the next interview transcribed by a professional agency to save some time. In the end, I transcribed the interviews conducted with my father and Burkard Rosenfeld myself whilst the audio scripts from Prof. Dr Schotten's and GB's interviews were transcribed by a professional service and then checked intensely for accuracy by me. In my view, whether the interview was transcribed by a third party or me had no influence on the intensity of my dialogue with the data since after the transcription I reviewed the data numerous times within different contexts. In addition, using a professional transcription service for one of my first interviews aided me immensely in my own transcriptions as it provided me with a template of a professionally-produced transcript (Flick, 2009).

Although I interviewed my father in August 2018, the other three interviews were all done in 2017 and transcribed in the first six months of 2018. I started to review the data intensely in November 2018. The long time slots between conducting the interviews, the transcriptions and the analysis, were partly the result of work commitments and a subsequent lack of time, but it was also a conscious decision of mine. To my mind, these time gaps in combination with working on other parts of my doctoral thesis permitted me to approach the data from a new perspective each time I immersed myself in them. In other words, each time - during the interviews, during the transcription process and during the coding stage - I discovered more or different aspects which I found interesting and relevant (Braun \& Clarke, 2006). Thus, this approach, I believe, furthered my reflexivity and subsequently the depth of the analysis and a thicker description of my participants' accounts (Flick, 2009; Geertz, 2008; Morley, 2008; Ritchie et al., 2013).

I had previously decided to follow a smooth verbatim transcription style in which I transcribed literally everything I heard even if there were grammatical errors, the sentences were not finished, or there were repetitions of sentences or words as well as habitual speech patterns such as "you know" "I guess" of both the interviewee and me, whilst I omitted fillers such as uhm and ahm, laughter and pauses. However, when I came across passages which I considered to be of particular relevance for the analysis, I listened to this particular part of the interview again not only to ensure that the interviewee's utterance was correctly transcribed but also to identify if there was laughter or a pause which could add an additional layer of meaning, such as when laughter is aimed at taking the sting out of something said 
or an exceptionally long pause indicated intense reflection on the participant's part (Flick, 2009).

The decision to exclude non-verbal communication markers and to explicitly focus on the transcription of what my participants said and not on how they behaved when they articulated their thoughts was mainly the result of one consideration. From the beginning of my empirical research until the end it was of crucial importance to me to show respect to my participants by being as transparent and trustworthy as possible in handling the data they generously and openly provided to me. This included avoiding reading sub-conscious meaning into the non-verbal signals, which accompanied the articulation of their thoughts (Silverman, 2016).

I do not prescribe to the view that everything we do when we communicate invariably reveals a frequently contrary, subtler and sub-conscious level to what is being said. Undoubtedly, facial expressions, laughter and body language add meaning to verbal exchanges; however, I often feel that interpretations of non-verbal communication by laypeople are somewhat random and frequently seem to be more motivated by supporting the personal preestablished evaluations of somebody than by being based on substantial psychological expertise. Since I am not a trained psychologist, I did not feel to be in a suitable position to judge what the behaviour the participants showed during the interviews might mean (Smith, 2015). In addition, my research aim was not to uncover some hidden mechanisms and structures my participants are unaware of but to better understand their life-worlds (Bhaskar, 1989; Comstock, 1982; Danermark et al., 2005; Harding, 1992). Indeed, I believed that had I focussed more on the non-verbal communication signals on the basis of my rather basic knowledge of behavioural psychology the interviewees might have felt cheated and somewhat deceived since I did not inform them about this level of analysis. As mentioned before, this was something which I wanted to avoid by all means since it would have jeopardised the truthfulness of the data and been ethically questionable and since it would have irrevocably damaged the relationship of mutual trust and respect between my interviewees and me (Silverman, 2016).

Similar concerns affected my decisions with regard to all issues linked to the translation of my interviews from German to English. In line with my research philosophy, I not only believe that language is, possibly more than anything else, bound to the socio-culture context one is part of, and it is spoken in but also that language frames and limits the construction of meaning since one can only express what one has words for (Foucault, 2000; Haraway, 1988). As such, communication is never neutral but a biased act of interpretation on the speakers as well as listeners part impacted by one's culture, social background, understandings and worldviews (Burr, 2006). It is, therefore, in my view, not exaggerated to 
say that although my interviewees and I come from the same socio-cultural environment and share the same language, the process of transcribing what my participants had said already involved a certain amount of interpretation on my part (Mason, 2017).

Although I attempted to transcribe what the interviewees uttered as precisely as I could and checked the accuracy of the transcripts several times against the audio script, I cannot exclude the possibility that there were occasions in which my own socio-cultural situatedness impacted my hearing capabilities and I, so to say, heard what I expected to hear. This is particularly true when utterances were ambiguous due to low volume, grammatical errors, background noises or mumbling which, however, and luckily, rarely happened. Nonetheless, and despite the fact that I invested much effort and time in being true to the data, I cannot entirely negate the potential that my own understanding of the world subconsciously impacted the transcription process. To minimise the danger of having made transcription errors, the participants counterchecked the transcription of their interview something that they had all ticked in the informed consent they had signed before the interviews took place (see Appendix A). Interestingly, except for my Dad, all interviewees invested a relatively high amount of time and effort in proofreading the transcript and said to me and others that they were surprised about how much they had told me. Corrections in the transcripts mainly concerned grammatical errors, eradication of verbal fillers and habitual speech patterns and errors in names or terminology. The content was not changed but occasionally smoothened, for instance, by adding 'less' and 'more' to sentences that were previously more declarative.

It follows logically, that if even the commonly viewed neutral process of transcription might be influenced by the person doing the transcription, this impact rises exponentially with every further step of the interview analysis. This is particularly true within the context of my specific research since it at some point involved translating all or some of the interview transcripts from German into English so that my English readership would be able to follow my analysis. Social constructionist discussions of the issues linked to translating any type of content from one language into another suggest that the act of translation will invariably lead to changes in meaning since every word in one specific language carries with it certain sociocultural connotations and historic and region-specific developments which cannot be transferred by directly translating words from one language into another. As such "there is no single correct translation of a text” (Temple \& Young, 2004, p. 165).

At some point, before the interviews, I had considered holding the interviews in English since all my participants are more or less fluent in the language. This would have solved the issue of translation but I, nonetheless, decided against it since I found it to be critical that the interviewees feel comfortable (Mason, 2017) - something which would not have been the 
case had they been forced to speak a language which was not their native language. Finding the right words for one's own emotion-laden experiences and understandings is often a challenge in one's native language and in a foreign language, regardless of how high the proficiency level is, accurately articulating what is on one's mind is near impossible since the array of words in a foreign language is much lower than in one's native language (Koulouriotis, 2011; Woodrow, 2006). Yet, gaining access to the life-worlds of my participants was my main focus, and I thus came to the conclusion that conducting the interviews in English would be counterproductive.

I, therefore, had to decide what needed to be translated and at what stage in the analysis. In positivistic oriented research, it is a commonly accepted standard to use literal word-forword translations of the entire transcript and to ensure validity and objectivity by backtranslation usually performed by somebody else (Regmi, Naidoo, \& Pilkington, 2010). Apart from practical issues such as the high amount of time needed for this approach towards translating, and the financial means needed to pay a professional translator for backtranslation, I did neither believe that this procedure would contribute to the depth of the analysis nor that it could sufficiently support staying truthful to the data (Larkin, Dierckx de Casterlé, \& Schotsmans, 2007; Temple \& Young, 2004).

On the one hand, despite the fact that I am a proficient speaker of English, my array of words, my knowledge of the grammatical and semantic structure of the language and its sociocultural connotations is naturally much more limited than in my native language and, on the other hand, a professional interpreter, even if I could have afforded one, would not have had any knowledge of my specific research context which would have impacted the equivalence of meaning in both languages (Regmi et al., 2010). To support the highest possible level of meaning equivalence, I decided not to translate the entire interview scripts but to postpone the act of translation as long as possible and to limit it to first capturing the main meaning of aspects identified previously in the before mentioned mind-maps (see Appendix K-P). This approach markedly expanded my understanding of the broad themes Subsequently, to support some access to my interviewees' understandings, feelings and experiences as older men, I selected relevant quotes illustrative for my participants' life-course narratives and translated these as literally and as closely to the original quotes as possible. When I found that the equivalence between the German quote and the English translation was too far apart and ambivalent due to the specific socio-cultural connotations linked, for instance to metaphorical language or proverbs well-known in Germany but not in the UK, I explained their specific meaning in the German culture in an annotation. In my view, this approach was useful as it enabled me to stay as close and truthful to the data as possible during the translation process (Birbili, 2000; Temple \& Young, 2004). 
When I had finished the transcriptions of the interviews, and before even thinking about the issues involved in translating the data, I was faced with the question of how to analyse the data. Being a complete novice in this field, I found this stage rather challenging and given my socio-cultural background yearned for some rules and regulations to keep my insecurities at bay (Hofer \& Pintrich, 1997; Scotland, 2012). Not surprisingly, I, therefore, decided to begin my analysis by feeding the data into Nvivo (Basit, 2003). This was quite timeconsuming since I did not really know how to use this computer programme and also since I did not really know what precisely the aim of my use of Nvivo was. At the first stage, I copied all transcriptions into Nvivo and then reflected intensely on the appropriateness of the themes defined and specified before and during the interviewing stage. I soon found that the broad themes of culture, masculinity, profession, ageing, retirement and private life were too open to be useful although it was effective for an initial approach towards analysing the data. At the same time, I realised that some of the more specific aspects I had defined as linked to these themes such as income, social status, successful ageing and ageing as decline, family, friends and hobbies aided me in refining the thematic units (Compare Appendix D). However, as a result of my continuous and intense dialogue with the data, I kept only some of these aspects, adapted others and removed a few entirely. In the end, I defined the following thematic units as the basis for coding the interviews: ageing as decline, successful ageing, control and autonomy, society/culture and media, masculinity and femininity, generational conflict and life course narratives.

However, when I started coding, I noticed not only that some passages were relevant for more than one thematic unit but also that nearly everything the participants had said matched the category of life-course narratives. With hindsight, this seems obvious since after all capturing the life course narratives of my participants was the main aim of my interviews, and since everything the respondents said was based on their life-course narratives. That I needed to go through the process of coding first to realise this flaw in the themes developed shows my inexperience and my initial confusion in identifying how the aspects covered in the interviews were linked to each other (Turner III, 2010). Indeed, the first stages in the analysis helped me immensely in gaining clarification about the different aspects involved in the construction of older male identity and in weaving these into one's personal life-course narratives which, in turn, aided the development of a codebook for the analysis of the interviews (Mason, 2017; Merriam, 2009; Patton, 2014).

The development of the codebook was an ongoing process which began with coding the interviews by applying all themes mentioned above in Nvivo. After that, I wanted to find a way to display what my participants had said with regard to each thematic unit so that I could compare what the interviewees had said. This would, in my view, be a practical approach 
towards capturing relevant aspects linked to each theme (Braun \& Clarke, 2006). After some experimentation with different functions of Nvivo, I managed to produce matrixes in which the utterances of my participants with regards to each specific thematic units were placed next to each other. This enabled me to gain a better overview of the similarities and differences in their responses. It was during this stage that I realised the impracticability of the theme life-course narratives (see above) and I thus decided to exclude this theme before continuing with the analysis. Although I had quite naively thought that the matrixes would be a sufficient basis for the analysis of the interviews and the subsequent write-up, I soon realised that there was still much too much data to permit a comprehensive analysis which provided insights into the lives of my participants whilst supporting readability (Basit, 2003). Hence, I needed to break down the content further.

Since mind- and concept maps had proved to be a handy tool to me during my doctoral research when attempting to gain a better cohesion of complex relationships or social phenomena, I opted for this strategy to further both, the management of data and my understanding of it (Mason, 2017; Merriam, 2009). This stage turned out to be rather timeconsuming and challenging as it not only involved much consideration of which content fit which theme and why but also as I had decided to use English for the elements which I believed to be related to each theme. Despite the fact that at this stage my aim was to translate the gist of what was being said and thus the focus was not on accuracy and on literal translation of quotes, I nonetheless spent a considerable amount of time to ensure that the meaning was not distorted which would have jeopardised the credibility and truthfulness of the data and, in fact, the entire research (Birbili, 2000; van Nes, Abma, Jonsson, \& Deeg, 2010).

At the same time, I moved flexibly between the concept maps and the codebook to further refine the elements which were linked to each theme and which I discovered during the development of the concept maps (Mason, 2017). In a final step, I compared the sub-aspects related to each of the six themes which I discovered, translated and recorded in the six concept maps with the transcripts of the interviews to ensure, on the one hand, congruence between the transcribed interviews and the sub-aspects discovered and, on the other hand, that I had not overlooked essential aspects. To additionally aid this process, I colour-coded all relevant passages in the transcribed interviews on the basis of the colours assigned to each theme in the codebook. This also enabled me to more effectively crosscheck the aspects discussed in the analysis with both the transcriptions and the concept maps while writing the analysis (Creswell \& Miller, 2000; Hammersley, 2007; Koro-Ljungberg, 2008; Miles \& Huberman, 1994; van Nes et al., 2010). 
The codebook consisted of six themes, namely 'Ageing as Decline', 'Successful Ageing', 'Control and Autonomy', 'Masculinity and Femininity', 'Generational Conflict/Intergenerational Cooperation' and 'Society, Culture and Media'. The theme of 'Ageing as Decline' included sub-aspects such as mental and physical decline, effects on identity and reflections on the finiteness of being and death. Aspects linked to 'Successful Ageing' surfaced mostly when the focus was on the professional and private activities of the respondents during their employment as well as when they had retired. 'Personal Autonomy and Control' seemed to mark a particularly essential aspect when the interviewees reflected on gender and sexual differences as well as when they talked about their professional and personal activities, which was the reason why I decided to cover these sub-aspects in a separate theme. Ageing predominantly gains meaning and is conceptualised by comparing oneself to other age groups. I, therefore, found it to be crucial to cover the aspect of 'Generational Conflict and Intergenerational Cooperation' in a separate theme. Similarly, what determines individual and socio-cultural understandings of masculinity is significantly influenced by comparing and contrasting it to the other sex(es) and gender(s). Thus, to gain insight into my interviewees' perceptions of masculinity, the respective theme needed also to cover the theme of femininity. Last but by no means least, there is no doubt in my mind that the socio-cultural setting and their agents majorly influence one's construction of the self through life-course narratives. As such, it was crucial to gain a better understanding of what my interviewees thought influenced societal and individual understandings of ageing and masculinity. The theme of 'Society, Culture and Media' was intended to enable capturing the respondents' own reflections (see Appendix F and K-P).

All in all, the themes developed proved, in my view, useful in supporting a thick description of what my participants had said (Geertz, 2008) and thereby facilitated a better understanding of their life-course narratives and their construction of identity in later life and as men. However, in hindsight, I realised that my determination to use Nvivo was motivated mostly by my very German need for feeling in control of the research process and for gaining undisputable, namely numerical data. One of the things which attracted me to Nvivo was, apart from the hope to ease the effort involved in the data analysis, that it could provide me with some factual data such as the coverage percentage of each theme compared to the entire interview. Initially, I was convinced that this would be useful and enhance the analysis. Yet, the further I proceeded with coding the data, the more I recognised that this kind of information would not contribute anything to the quality of my analysis.

In fact, I believe, the inclusion of these percentages would have been counterproductive since it would have implied that there is a correlation between the number of words somebody uses to discuss any particular issue and the significance of what is said. In other words, it 
would have indicated that the more my participants talked about any one aspect, the more important it was for them and the more important it should, therefore, be in the analysis. Although in some instances there might be some truth in this, in all I find this assumption dangerous and misleading since how much one talks about something is dependent on numerous other factors such as character, familiarity with the topic or the physical and mental state of the participants on the day of the interview. Indeed, it could very well be the other way around and the less and more precisely someone talks about an issue the more it shows how much the participant has thought about a particular topic. In any case, since I did not believe that coverage rates would add depth to my analysis, I excluded them and generally came to the conclusion that Nvivo is not an effective tool for research grounded in a social constructionist research paradigm (Basit, 2003).

When I started writing up my analysis, there were mainly two issues I experienced as challenging. Firstly, all themes developed were closely linked to each other and combined contributed markedly to the life-course narratives of my participants. To separate the data and allocate their testimony to themes was necessary to manage the data, which was the prerequisite for producing a coherent, thick and readable report. At the same time, it was essential to link the data with each other and draw references between and across the themes since only then was it possible to explore the depth of the data sufficiently and gain a holistic understanding of the interviewees' perspective on the issues discussed (Geertz, 2008; Mason, 2017). This made a certain amount of repetition unavoidable, which might have decreased the readability of this thesis. However, I believe that the gain in-depth justifies the slight increase in the effort it will take to read the analysis.

Secondly, what I found to be an ongoing and somewhat insurmountable challenging during all the stages of the empirical analysis was to keep an open mind by attempting to not let my own views on the construction of identity in older male age, which were partly the outcome of having researched the socio-cultural construction of ageing and masculinity in-depth and partly of previous observations and experiences, influence the analysis. I have been adamant about justifying the trust the participants credited me with by answering my questions openly and extensively by aiming for a non-evaluative, holistic and rich report in which the respondents feel represented. This required me to move beyond my own cultural embedment firmly situating me in a research context majorly influenced by a positivist research orientation in which research is, despite claiming to be objective, frequently characterised by dissecting what interviewees said to uncover hidden meanings and to confirm predetermined categorisations and perceptions. Since one is often not aware of how strongly the socio-cultural beliefs and standards of the country one lives in influence oneself, my worry was that I would without even noticing, start to make the data fit my own assumptions 
and understandings of the research context (Cooper \& Hedges, 2009; Denzin, 2001; Guba \& Lincoln, 2005; Schommer, 1990).

To consistently maintain a researcher position of neutral empathy during the process of analysis has been a continuous struggle which required me to permanently reflect on what I was doing and writing (Patton, 2014). What aided me immensely was that one of the participants was my father and the constant thought of how the participants and particularly my father would receive what I had written. Under no circumstances did I want him or any of the other interviewees to feel offended or hurt by what I had written whilst I also wanted to avoid tampering with the data to please him and the other three interviewees. To facilitate my neutrality and truthfulness to the data, I stayed as close to the data as possible by supporting the analysis with direct quotes of the interviewees whenever suitable (Creswell \& Miller, 2000; Geertz, 2008; Mason, 2017). I was motivated by the hope that the participants would when reading my doctoral thesis feel that my analysis reflected their lifecourse narratives and perception of ageing and masculinity accurately and in-depth. An ambitious aim I sincerely and adamantinely attempted to reach. 


\section{Reflections of Four Retired German Men}

\subsection{The Interviewees}

From the beginning of my doctoral project I perceived the selection of the interviewees as one of the most critical decisions to be made (Flick, 2009; Grix, 2010; Miles \& Huberman, 1994; Patton, 2014). Resultantly I spent a high amount of time thinking about who would be suitable and how I could identify and contact potential participants. During the initial stages when I was still very much, although sub-consciously, firmly bound to the positivistic research orientation in Germany, my thoughts mainly circled around how to find a sample which would somehow be representative for what I was trying to find out (Patton, 2014). I was considering a heterogeneous sample and conducting about ten interviews in which scenes of the films I had selected would be shown to the participants to stimulate discussion. I had planned to recruit suitable participants by posting calls for participation on websites and online forums targeted at older people.

In all honesty, I have to admit that I neither felt comfortable with the way of contacting the participants nor with conducting interviews with men I did not know at all. In addition, I felt very insecure in determining criteria for the interviewees' selection and repeatedly asked myself questions such as 'Do all participants need to have seen the Expendables trilogy? Do they need to be outright fans of action films or not? What ages and social background should the participants have and why?' Nonetheless and despite my struggles with this research approach, I did in this phase not consider alternatives since I believed that this is how you do and go about proper scientific research and that the problems I had with it were a natural part of scholarly work (Golafshani, 2003; Hofer, 2000).

Thankfully, the further my research continued, the more it dawned on me that my views on how to conduct valid scientific research were a result of my socio-cultural embedment. Realising that there was not only good and bad research but that how one conducts scientific projects is largely dependent on one's ontological and epistemological views was a revelation (Guba \& Lincoln, 1994; Schommer-Aikins, 2004). It allowed me to much more intensely consider what my research interests actually were and what I thought to be a suitable way to gain more knowledge in these areas instead of thinking about how research should be, according to some omnipresent authority, conducted to be valuable. Taking on a social constructionist research stance aided me in recognising that I neither believed in the possibility of objectivity and undisputable representativeness, nor was I interested in it. I much rather wanted to understand the world, its processes and individual experiences with it better (Guba \& Lincoln, 1994; Haraway, 1988; Harding, 1992; Schommer-Aikins, 2004). 
As a consequence, my research changed its focus and now aimed at exploring the experiences of older men in Germany with retirement and resulting adaptation to their lifecourse narratives. What I was particularly curious about was how men, who during their professional life in many ways represented the embodiment of the socio-cultural standards set for hegemonic masculinity in current western society, handled their retirement and the process of getting older. This was, without a doubt, mainly a consequence of my personal background and the observations I had made in relation to my father's retirement and his coming to terms with this new phase in his life as an ageing man.

Within this context, it must be mentioned that my interest lay in identifying how older German men who perceived themselves and were perceived by others as embodying many aspects linked to hegemonic masculinity constructions in the West use the socio-cultural frames of successful male ageing revealed in films such as The Expendables to construct their identity as older men. It was not about understanding of how men who resemble the on-and offscreen personae of the protagonists in The Expendables trilogy, such as bodybuilders or martial arts fighters, handle the ageing process. Thus, the selection of my interviewees was based on identifying participants whose mental and physical fitness permitted them to continue doing activities such as sports which had during their life stages determined their status within hegemonic groups of men. I viewed this as essential in understanding how continued mental and physical fitness might play a role in my participants' identity construction in older age and how their views reflected the criteria linked to successful male ageing.

But how was I to locate interview participants who matched the criteria outlined above? I considered following the original approach of using websites and forums to identify and recruit potential participants but somehow still felt uncomfortable with this strategy since not knowing the interviewees at all would make it very difficult to develop rapport and trust which, in my view, was a prerequisite for gaining insights into their life-course narratives as older man (Blichfeldt \& Heldbjerg, 2011). In addition, I was worried about the consequences my research might have on the participants. In my view, there was some likeliness that older men who spent much time in online forums and on websites and would be willing to support me with my research might potentially be rather lonely and could be longing for some social recognition. This might have led to an emotional overreliance on me and resulted in disappointment and potential emotional suffering on my interviewees' part once they realised that my interest in them was professionally and not personally motivated (Denham, 1984; Russell, 1999; University of Gloucestershire, 2008). I had already experienced similar situations when I taught older men English in one-to-one tuitions and had, at that time, found it rather challenging to deal with their personal interest in me and their emotional attachment 
to me. After discussing these issues with my supervisors as well as with family and friends, I, therefore, decided to attempt finding research participants in and through the broader circle of my friends and family.

In the end, all interviewees I selected were part of my family's wider circle of friends whom I had met, at most, a couple of times but never talked to at lengths or in-depth. Nevertheless, I benefitted greatly from the sympathy and respect they felt for the members of my family they were closest to since they extended this trust to me before the interviewing process had even started. Not only did they all, without any hesitation, agree to participate in my research, but they also showed great enthusiasm for and curiosity about my project. Their willingness to cooperate with me markedly eased the establishment of rapport and enabled a research collaboration on eye-level which, I believe, significantly contributed to the depth and richness of the data received (Patton, 2014; Robertson \& Hale, 2011). Moreover, having some knowledge of the socio-cultural background and professional development of the interviewees permitted me to tackle the issues which interested me rather directly whilst being aware and showing some sensitivity towards areas in which the participants had made painful experiences, such as having lived through the death of a partner or other family members, divorce or serious illnesses. At the same time that the interviewees were acquaintances of my family, and particularly my father also meant that I did not want to disappoint or upset the participants in any way. Although I believe that this was a useful corrective for staying neutral and truthful to the data, it also raised the pressure on me markedly (see section 6.2 and 6.3). Nonetheless, I am happy with my decision since the selected approach permitted me to explore the issues of ageing and masculinity in a level of depth which would not have been possible, had I chosen another approach.

All interviewees have been exceptionally successful in their careers, are white, heterosexual and married with children - criteria which firmly mark them as belonging to the hegemonic masculinity ideal of corporate masculinity promoted in the West (Cheng, 1999; Connell, 2005; Connell \& Messerschmidt, 2005) - and are now officially retired. Prof. Dr Schotten was born in 1938 and at the time of the interview 79 years old. My father was born in 1939, whilst Burkard Rosenfeld was born in 1941 and GB, the youngest participant, was born in 1946. They all grew up in Germany, finished school with the qualification allowing them to study and all except Prof. Dr Schotten, who was exempt for health reasons, then served between fifteen months and two years in the German army - something which was obligatory in Germany until 2011. Burkard Rosenfeld studied mechanical engineering at a vocational college in Munich and then continued his education with a post-graduate course in economics. Prof. Dr Günther Schotten studied law in Innsbruck, Heidelberg and Bonn and then studied philosophy since he had achieved his state examination rather early. He 
then proceeded to do his doctorate in international law. Dr Lars Bühring studied economics in Marburg and then completed his education with a doctoral thesis which was subsidised by the Volkswagen foundation whilst working for the Max-Planck institute in Bad Godesberg. After finishing his obligatory military service, GB started his vocational training in a branch of Kaufhalle - a German department store which belonged to the Kaufhaus group (Rosenfeld, Personal Communication, 17.07.2017, Schotten, Personal Communication, 18.07.2017, GB, Personal Communication, 15.12.2017, Bühring, Personal Communication, 31.07.2018).

After Prof. Dr Schotten had finished his education, he took over his father's notary office in Monschau since he had fallen seriously ill and then passed away. In 1984 he moved to Cologne and continued his activity as a notary. In addition, he became an honorary professor for law at the university in Bielefeld and an examiner for the second state exam for lawyers. Moreover, he published academic articles on legal issues, revised the Staudinger, an influential commentary on the German Civil Code, every five years, conducted advanced training courses for lawyers and notaries on private international law and was a tutor in the chamber of notaries (Schotten, Personal Communication, 18.07.2017).

Burkard Rosenfeld started his professional career at the Technical Association for Steelmaking in Düsseldorf in the R\&D department for one year and then served his obligatory service in the German army. He subsequently took on a position as deputy chief in the R\&D department of the machine factory Weingarten in Weingarten. He was promoted to head of material testing and quality assurance but left the company after four years since it had manoeuvred itself into a difficult financial situation. In the next 18 years, Burkard Rosenfeld took on positions as deputy head of investment, vice president of technology and managing director in international as well as national companies in Coburg, Essen and Leonsberg. In 1990 he joined the company Indus Holding in Bensberg near Cologne as a member of the management board responsible for all issues related to technology. In 2008 he became a member of the supervisory board of Indus and then took on the position of the chairman of the supervisory board from 2009 until 2014 (Rosenfeld, Personal Communication, 17.07.2017).

After GB had finished his traineeship as a sales manager, he moved up the career ladder very quickly. Within 14 years he first became a senior buyer for baby products, then for leather products and finally, he was promoted to sales director for all textiles. As a result of the Kaufhalle having been taken over by Metro, he took on the position of senior buyer in Asia for the Metro Group. During this time, he commuted between Hongkong and Metro's headquarters in Switzerland. In 1996 he returned to Germany and headed the newly founded Cash and Carry Holding of the Metro for non-food. In 2003 GB became the chief 
representative for corporate development and internationalisation. In addition, he was active in the Chamber of Commerce as chairman of the foreign trade committee and was a lecturer and tutor for internationalisation and project management at the university in St. Gallen (GB, Personal Communication, 15.12.2017).

After having finished his doctoral thesis, Dr Lars Bühring resigned from his position at the Max-Planck Institute in 1970 and began working as a controller for the large multinational company Esso in Hamburg and stayed there for three years. From 1973 until 1988, my father worked in different positions for a medium-sized company, Otto Wolff, with a focus on metal processing in Cologne. A significant part of his duties at Otto Wolff was to travel to countries in Asia, Africa and Latin America. After he had successfully completed his training as a tax expert, my father decided to join a former colleague of his as the Chief Financial Officer. He was the second member of the management board and established a start-up company with his colleague which bought highly specialised companies in Germany whose owners did not have a successor but wanted to ensure that the firm was taken over without significant changes to the structure and philosophy of it. This concept was highly successful, and Indus became a public holding company in 1995. After my father retired from his position at Indus in 2008, he became a member of the board in a private foundation and then took on the position as the head of the executive board in this foundation in 2017 (Bühring, Personal Communication, 31.07.2018).

Prof. Dr Schotten, Dr Lars Bühring and GB grew up in a middle- to an upper-middle-class socioeconomic background. Burkard Rosenfeld was the only participant who experienced some poverty in his childhood and also the only one who was reared in a single-parent household. He and his younger brother were brought up by their mother since their father had been killed in action in the Second World War. The other three participants spent their childhood and adolescence in so-called intact families, in which the parents stayed with each other their entire life, and their relationship resembled the, at their time, conventional role division between women and men. As such, the mother stayed at home and took care of the children and the household and the father took on the role of the breadwinner. Lars Bühring grew up with two considerably older sisters, GB had three older brothers who are each one year apart and Prof. Dr Schotten has a younger sister whilst Burkard Rosenfeld had a younger brother who has already passed.

Dr Lars Bühring and GB are divorced and married for the second time. My father's second wife is about ten years younger than he is, and GB's current wife is about fifteen years younger than he is. Prof. Dr Schotten has been married for fifty years, and his wife is slightly older than him. Burkard Rosenfeld lost his first wife through illness and is now married for a second time to a woman who is eight years younger than him. GB has two children with 
his first wife, both sons, and a daughter and a son with his second wife whilst Dr Lars Bühring and Burkard Rosenfeld both have one daughter from their first marriage. Prof. Dr Schotten has two children, a son and a daughter (Rosenfeld, Personal Communication, 17.07.2017, Schotten, Personal Communication, 18.07.2017, GB, Personal Communication, 15.12.2017, Bühring, Personal Communication, 31.07.2018).

As could be seen, although all interviewees match or used to match the majority of the sociocultural standards western conceptions of hegemonic masculinity are characterised by, they also differ considerably in many aspects. Not only their career developments, their professional orientation and resultant experiences deviate but also their personal background and their life-course developments vary more or less strongly. In my view, the combination of similarities and differences led to a particularly interesting and promising research context. Despite the fact that relatively often the answers of the interviewees were somewhat similar, there were also many occasions in which their views and experiences were sometimes slightly and hardly noticeable and sometimes markedly differed. This, in my view, contributed considerably to the depth of the data and thereby significantly furthered a better understanding of how these four German men experience the process of ageing. It also facilitated gaining insights into how they construct their life-course narratives within and around the socio-cultural frames available for older men to construe their identity in older age (Haraway, 1988; Harding, 1992).

\subsection{Reflections on Masculinity, Femininity \& Ageing}

Ageing is a process, and as such one does usually not wake up and perceives oneself as old. It is a gradual, often hardly noticeable development which, at least in my experience, in western societies begins at some point around forty. In fact, most people only become aware of getting older and of being increasingly perceived as belonging to the group of older people by both comparing oneself with others and by the social feedback one receives (Gullette Morganroth, 2004; Gullette Morganroth, 2008). In other words, seeing friends and acquaintances getting married, having children and observing their own and their parents' ageing process, watching celebrities on TV and in films age or realising that certain ads targeted at older people actually target oneself lead to an increasing awareness for one's chronological age. At the same time, hearing comments such as 'You look much younger than you are' or 'I would not have thought you are that old' slowly results in feeling and defining oneself as old even if often in combination with believing not to be, behave and look quite as old as other people in a similar chronological age (Segal, 2014). As such, age is a highly relational concept mostly dependent on one's socio-cultural context (Baars, 2013, 
2015). Resultantly, coming to terms with older age and adapting one's life-course narratives to age-related bodily changes as well as to the alterations to one's social status and life-style in older age is an ongoing activity starting at middle age and often continuing, I believe, until the time one passes away.

Hence, I assumed that men's conception of what characterises masculinity has not changed markedly from the time they were younger to the time when I interviewed them. As such, the interviewees' definition of masculinity is, in my view, a, if not the most fundamental basis for gaining a better understanding of many of the other aspects related to ageing and particularly male ageing discussed in the interviews (Bourdieu, 2001; Butler, 2011). This was the reason why I decided to start the interviews by asking the interviewees to define masculinity, and it is also the rationale for including a translation of their entire answer to this question.

Burkhard Rosenfeld described his perception of masculinity as follows:

To me, masculinity means to have the ability to take part in reproduction and play a crucial role in it. Masculinity is also linked to this traditional understanding of the male respectively female role. That is that the men of the cave people went hunting and the women were responsible for the part, which took care of the family. The man provides the family with protection, and the woman provides emotions and feelings (Rosenfeld, Personal Communication, 17.07.2017, p. XXIX, line 3-8).

Prof. Dr Günther Schotten replied when asked what for him determined masculinity: "Well, masculinity means to me a...the attempt to think stringently ${ }^{3}$ and also a certain amount of physical effort and a strong reference to rationality, a highly rational life," (Schotten, Personal Communication, 18.07.2017, p. LIII, line 3-5). When I then enquired if these characteristics must be viewed as in opposition to female attributes, he confirmed that by saying: "To a certain degree, yes," (Schotten, Personal Communication, 18.07.2017, p. LIII, line 7) GB's answer to the same question was rather lengthy, but I nonetheless decided to include his full answer since I believe it to be particularly insightful:

Honestly, I have never really thought about this. Well, to me masculinity is somewhat a little more linked to a question mark than to an exclamation mark. Since I was -, you need to see the individual situation, so we were four boys at home, and I was the youngest, and I was, let's say, not only by age but also by nature the most sensitive of the four. And the others were bigger, stronger and rougher, I was somewhat more highly sensitive, what then somehow was, yes, somehow perceived, by my social surroundings more than by me, I don't want to say, to have a more feminine touch but it was not the kind of masculinity, let's say, that was embodied by others. I was also more artistically talented whilst my next oldest brother was the sporting ace, he played football. The one before that one was somehow the craftsman and the oldest was, well, the oldest. As such masculinity was somehow embodied by, let's say, strength, powerfulness, dominance, a man of action type, but I somehow viewed this perception

\footnotetext{
${ }^{3}$ The German word 'stringent' is linked predominately to notions of coherence, logical thinking and rationality and not as in English to being strict or forceful
} 
with a question mark since by nature I was not like this at all. So I had a conception of what masculinity was but, let's say, also some healthy doubts about how sustainable and also justified this was, let's say, simply because of the discrepancy to my own feelings, to how I viewed myself (GB, Personal Communication, 15.12.2017, p. LXXVII, line 6-22).

Dr Lars Bühring defined masculinity as follows:

Masculinity means drive, determination, well, another word, activity, to be responsible for others. Ultimately to be able to also live with the consequences, which in his surrounding in some way have significance and to identify himself with them and to derive, well, then the suitable consequences and activities. Well, really it is the opposite of passive (Bühring, Personal Communication, 31.07.2018, p. CVIII, line 2$6)$.

Interestingly, although in some ways similar, all four men stressed different aspects of masculinity. Prof. Dr Schotten emphasised rationality and physical activity. In fact, later on, he specified his definition of masculinity by referring to the "problem of masculinity" which he defined as "performance orientation with a strongly systematic approach," (Schotten, Personal Communication, 18.07.2017, p. LVI, line 3-4). Some minutes later the topic came up again, and Prof. Dr Schotten referred to his wife who introduced the idea of the "masculinity gene" which she defined as being "performance-oriented, Yang-excess, to prove oneself, that you can still do it and so on," (Schotten, Personal Communication, 18.07.2017, p. LXII, line 18-21). Burkard Rosenfeld and my father both followed a similar train of thought when the former suggested that masculinity had been determined in early human history when the men were the hunters and protectors of the family while the latter named, among other attributes, activity as a core element of masculinity. GB's extensive answer was particularly fascinating since he mentioned what others link to masculinity, such as strengths, dominance, roughness, sportiness, to name but a few but clearly distanced himself from this view since it did not match his own personality.

The emphasis on activity, logical thinking and performance reflects a hegemonic cultural narrative grounded in biology and evolutionary theory which, as discussed in section 4.1, communicates an ambiguous understanding of masculinity. On the one hand, the binary between feminine attributes such as emotionality, passivity and being caring and masculine attributes such as rationality, activity and goal orientation is through and in cultural agents, such as the media, academia or the legal system, established as determined by nature and therefore unchangeable (Butler, 2011; Fausto-Sterling, 2000; Lorber, 1996). On the other hand, research in endocrinology in the 1950s, which revealed that the development of a male embryo requires much effort, given the fact that all foetuses are initially female, has crucially contributed to the solidification of the belief that being a male is a challenging and life-long endeavour. After all, although the characteristics commonly viewed as masculine are 
genetically pre-determined, staying a man and not being taken over by female hormones apparently takes considerable work (Fausto-Sterling, 2000, 2008).

That all participants, even GB who was in the above quote rather sceptical towards conventional understandings of masculinity, are impacted by conventional understandings of the male-female divide is also evidenced by the fact that all interviewees more or less strongly stressed the differences between men and women. Burkard Rosenfeld said strikingly little about the specific characteristics of women, but at one point when we discussed his favourite sport, golf, he said: “... for men it is about being sporty, they might cheat a little bit more but besides... women have ambition and clothes and Andiamo ${ }^{4}$," (Rosenfeld, Personal Communication, 17.07.2017, p. XLI, line 14-15 \& p. XLII, line 1). When I asked if in his view men or women suffer more by the ageing process, he said: "I think on average women suffer more by the physical signs of ageing, wrinkles, glasses, whatever," (Rosenfeld, Personal Communication, 17.07.2017, p. XLVIII, line3-4). This view was also entertained and elaborated upon by my father, who in reply to the same question, said:

It is much more difficult for women...Yes, because I think that on the one hand, in a different age, they, at least in my view and I think are much more linked to their social environment in a specific way, well, integrated in their social environment, you could also say more dependent and therefore more dependent on the echo ${ }^{5}$ of their social surroundings than it is the case with men. And also since the appearance plays a crucial role and the echo naturally becomes less when one becomes a little older. That means they have with ... I think that is; they will have more difficulty in keeping their self-concept (Bühring, Personal Communication, 31.07.2018, p. CXXVIII, line 3 \& line 5-12).

Neither, Prof. Dr Schotten nor GB agreed with this conception of women, particularly of the women they know. Prof. Dr Schotten first said when I asked him whether ageing was more difficult for men or for women: "I have heard that it is more difficult for women," (Schotten, Personal Communication, 18.07.2017, p. LXXIV, line 4). When I then probed for more detail by asking him if he thought that was true, he replied: "I don't know. I don't think so. It's not the case with my wife. She as little a problem with getting older as me. You hear that regularly but in my circle of friends I cannot confirm that," (Schotten, Personal Communication, 18.07.2017, p. LXXIV, line 6-9).

Nonetheless, in other parts of the interview, both GB and Prof. Dr Schotten reflected on the differences between men and women. In addition to mentioning the 'male gene of performance orientation' (Schotten, Personal Communication, 18.07.2017, p. LIX, line 78), Prof. Dr Schotten's view of the natural role division also became apparent when he talked

\footnotetext{
${ }^{4}$ Since Burkard Rosenfeld is fluent in Italian, he here used the Italian word for 'Let's go'

5 'Echo' in the German language can be used to describe social recognition
} 
about his wife's professional occupation. He described to me that his wife used to have a lawyer's office with a focus on divorce, inheritance, corporate and criminal law relating to young offenders which was rather successful “...but small. Very small and it always took backstage. The children had time-wise always preference, and when there was something ...That is why it $^{6}$ did not play such a big role for her," (Schotten, Personal Communication, 18.07.2017, p. LX, line 2-4). And shortly after he remarked: "My wife worked much but, as said, always totally in the background and very little. In comparison to me naturally very little," (Schotten, Personal Communication, 18.07.2017, p. LX, line 6-7). I then asked: “And she took care of the children?" and Prof. Dr Schotten answered: "Exactly, that always had preference, which was appropriate. ${ }^{7}$ That is why she called herself a single mother," (Schotten, Personal Communication, 18.07.2017, p. LX, line 9-10).

GB repeatedly mentioned his high respect for women and especially for his mother. For instance, he said: "Well, I have never met a woman who worked as much as my mother," (GB, Personal Communication, 15.12.2017, 15.12.2017, p. LXXXI, line 21-22). When I enquired about the gender-specific handling of the ageing process, he even said: “...I also believe that women have much more inner balance... Within this context, I have always been somebody who said that women are the stronger sex," (GB, Personal Communication, 15.12.2017, p. CVI, line 9-10 \& line 14-16). Nevertheless, it became clear in his definition of masculinity that regardless of his high respect for women, he did not want to be affiliated to female characteristics when he said: “...I don't want to say, to have a more feminine touch but it was not the kind of masculinity, let's say, that was embodied by others," (GB, Personal Communication, 15.12.2017, p. LXXIX, line 12-13).

In my view, the above responses show that the interviewees have incorporated a conception of gender characteristics based on sexual difference into their identity construction in which characteristics such as activity, performance orientation, being driven by ambition for professional success and the will for domination and winning are attributed to masculinity whilst female characteristics are generally linked to being caring, being more balanced, being emotional and warm, being less interested in career progression but also to passivity and, to a much lesser degree, dependence. The answers, however, do not indicate a general disrespect for women but simply show that women were perceived as a totally different species which has little in common with men. In fact and despite what is suggested by some scholars (Connell, 2009; Kimmel, 2001; Schippers, 2007), at least, Prof. Dr Schotten,

\footnotetext{
${ }^{6}$ her job

${ }^{7}$ The interviewee used the word ,richtig' which literally translated would be 'correct' but within this context he referred to the appropriateness of behaviour in his eyes and not some objective standard of correctness. I therefore chose the word 'appropriate' instead of 'correct' in the translation
} 
Burkard Rosenfeld and GB did not appear to link feminine characteristics to negative connotations but instead seemed to, in different degrees, feel admiration for women and their way of handling life. Within this context, it is also particularly interesting that women who in some way display male characteristics were not, as Schippers (2007) believes, categorised as unnatural or social pariahs. In fact, it neither seemed to be something which the interviewees viewed as unlikely nor did it seem to impede their appreciation or respect for these particular women.

Burkhard Rosenfeld said when reflecting on the fact that his first wife had only found a satisfactory professional occupation rather late in her life whilst his second wife had continuously worked part-time in a pharmacy during their relationship: "But after all, she ${ }^{8}$, she, after all, had additionally ${ }^{9}$ had a job, not full-time but part-time. That somewhat gave her strength, and that did not exist for my late wife," (Rosenfeld, Personal Communication, 17.07.2017, p. XXXV, line 24-26). At another point during the interview, Burkard Rosenfeld described his current wife as "... a little more curious than me," (Rosenfeld, Personal Communication, 17.07.2017, p. XLV, line 6-7). These remarks and in general the whole conversation left me with the impression that it was of particular significance for Burkard Rosenfeld to be on eye-level with his wife and that he believed to be equal partners in the relationship was a prerequisite for a healthy relationship and a working marriage.

Similarly, Prof. Dr Schotten stressed that he and his wife were intellectually on the same level and that this markedly increased the quality of their lives, also in older age: “...And yes, my wife and me, we have well, ...We also get on intellectually $100 \%$. We are nearly always of the same opinion. Also critical, very critical. We really are individualist somehow. But also, very critical about all and nothing and as such it ${ }^{10}$ has been quite good," (Schotten, Personal Communication, 18.07.2017, p. LXIV, line 9-12). He also mentioned that he still had contact with two female colleagues who frequently contacted him to discuss legal issues. He clearly held these women in the highest respect and did not at any point indicate that he found it remarkable or inappropriate when women worked in the same profession as he used to. Referring to one of these two women, he said: “...We are on an absolutely identical wavelength. When she has questions, they are always absolutely justified...," (Schotten, Personal Communication, p. LXII, line 12-13).

GB was, except in the instances already quoted above, a little less explicit about his opinion about women who display characteristics and abilities traditionally linked to men. I, however, believe this was based on him not even questioning that women have the same and

\footnotetext{
${ }^{8}$ His second wife

${ }^{9}$ In addition to being a mother and housewife

${ }^{10} \mathrm{It}=$ retirement respectively the quality of life during retirement
} 
perhaps more abilities than men - an understanding which he transmitted throughout the interview (GB, Personal Communication, 15.12.2017). The implicitness with which he took the equality of men and women for granted is, I believe, the result of his upbringing on two counts. First of all, he is considerably younger than the other participants and therefore grew up in a time characterised by civil rights movements and feminism which certainly influenced him and his views on gendered hierarchies (Baur \& Luedtke, 2008; Brown, 2013; Kimmel, 2012). At the same time, the admiration he felt for his mother, a woman who not only took care of her family and a household but also helped his father considerably with the administration of the company, very early presented him with a female role model highly unusual for that time. His slightly different view on women became utterly clear to me when he, as the only participant, said quite casually: "That therefore means, everything which was family, what had to do with the children was her job," (GB, Personal Communication, 15.12.2017, p. XCVI, line 15) At first sight, this might seem to be a rather conservative remark referring to the traditional role division between men and women, and partly this might be true, but to define taking care of the family and the household as a job and thereby equating it with paid jobs, I find rather unusual.

Although my father, to my mind, provided the most conventional and rigid definition of masculinity in which he somehow implicitly excluded the possibility of women having the same abilities as men, this was relativised in his answer to my follow-up question. When I inquired if he believed that the qualities he mentioned as being characteristic for men could also apply to women, he admitted that that was possible but not as likely as with men. Precisely I asked if his definition of masculinity was also based on a clear demarcation from women and my father answered: "Largely yes, absolutely. But, of course, I also know women to who this demarcation only applies to, to a limited degree..." (Bühring, Personal Communication, 31.07.2018, p. CVIII, line 9-11). In addition, I know that my father, in general, admires drive, competence and dedication in people but, as far as I can judge, regardless of sex. In fact, in the above-mentioned foundation, he cooperates with several women who are either with him on the managing board or work with the foundation on planned projects. When he talks about his activities in the foundation, I have never noticed that he made a difference between the men and the women he worked with, or that he felt it to be unusual, unnatural or threatening when he collaborated with women (Personal Communications at different times between Lars Bühring and Lisa Bühring between 2015 and 2019).

Without a doubt and clearly not the least as a result of the historical context they grew up in, all interviewees understand masculinity by and large in contrast to femininity. They entertain a rather conventional view of the male-female role division whose articulation is nowadays 
evaluated as politically incorrect which, however, does not mean that men regardless of age do not think similarly. Younger men and women might predominantly just not dare to utter this in public to avoid social sanctions (Bourdieu, 2001; Butler, 2011; Hall, 2000, 2002). Nonetheless, the interviewees' views on gender relations and their own embodiment of masculinity are neither quite as black and white and inconsiderate nor as unambiguous as it may seem at first sight. This became particularly obvious not only in above comments on their conception of femininity but also at two other points during the interviews, namely when I asked the interviewees about what they thought their conception of masculinity was based on, and when the interviewees reflected on their priorities during their employment.

Prof. Dr Schotten and Burkard Rosenfeld attributed their understanding of gender roles explicitly to their specific situation during childhood and adolescence. Prof Dr Schotten said:

I think that one is somehow influenced by one's parents, grandparents, by what one experiences. I believe that. Also, by the traditional role model. That was much stronger than one can understand today. ... Yes, my parents stayed together all their lives. And my father worked. He was a notary, too. And my mother took care of the children. She was very extramural, was involved in politics, was in the city council, in the unitary council, but in the end, the role division was absolutely there (Schotten, Personal Communication, 18.07.2017, p. LIV, line 1-4 \& line 6-9).

Burkard Rosenfeld elaborated on his view of the man being the provider and protector of the family when I asked him about his priorities during his professional life:

I was influenced by my family background. ... So, she had, my mother had, with 29 the war ended and with 32 she found out that she is a widow and there she had two boys and had under great privations to bring up these children. That influenced me so that I wanted to become independent as soon as possible; I really wanted to earn my own living. And then could not only earn my own living but I could also together with my brother take care of my mother, who was a fantastic person. And that obviously influenced me ... I take care of the protection of my family. And my wife takes care of the family itself, of the warmth of the family...(Rosenfeld, Personal Communication, 17.7.2017, p. XXXIII, line 2-3 \& line \& line 10-18).

GB had already in the above-quoted response to his understanding of masculinity indicated that although he was sceptical about the masculinity conception, he saw embodied in his social surrounding, one's understanding of what masculinity is primarily influenced by the specific individual situation. As such, I believe it to be justified to say that GB's somewhat relatively contemporary and ambiguous perception of masculinity is the consequence of his younger chronological age but also of what he saw exemplified by his brothers, by his parents and particularly by his relationship to his mother. When I asked him with whom he had a closer relationship, his father or his mother, he answered: "Clearly to my Mom. I was somehow her Benjamin...I was very close to her, also later, even as an adult,'(GB, Personal 
Communication, 15.12.2017, p. LXXX, line 4 \& line 9). Soon after he then described the role division in his family:

Well, my mother was the centre of the family. My father worked from morning till night...but still had when he came home the half hour to play football with his sons. But my mom was the soul of the family. She managed everything, did everything. She was there for everything and everybody.... a well-fortified woman. But nothing took priority over her family. And, well, I mean, what you might as an adult when you look back, view a little more differentiated, for her, the children came first and then there was nothing for a while, then came her husband and then came the rest of the family...(GB, Personal Communication, 15.12.2017, p. LXXX, line 12-13 \& line 1418 \& p. LXXXI, line 1-3).

When I asked Dr Lars Bühring if he thought his understanding of masculinity was influenced by his upbringing, he denied this possibility and said that the older he became, the more he made his decisions without consulting his parents or siblings. He then continued:

That's why this conception ${ }^{11}$ really formed itself slowly through this development and was not like this from the start. When I look at my mother, then this impression would largely be attributed to the fact that my mother was in some way very passive, but during the war, she incredibly determinedly guided the family's destiny. And with regards to my sisters, they then were in different degrees able to have their own activities, set their own course to then in the end in some way subordinate themselves to the general standards, that means their respective husbands. Insofar I think that throughout the years it formed itself slowly and well, less during adolescence (Bühring, Personal Communication, 31.07.2018, p. CVIII, line 14-24 \& p. CIX, line 1-3).

When I followed this comment up by asking if his father, therefore, did not match his conception of masculinity, he answered:

He did match it, but ${ }^{12}$ he did match it. But I don't know if he really was a role model for me and I, therefore, adopted this story. Because this role model or let's say what my father represented resulted from a time that was partly the imperial period. There was a completely different reason than activity or something like that. That was more authoritarian. I don't know if I wanted to perceive it like that. That wasn't really a role model which I... I saw that my father was very much in charge ${ }^{13}$ but I then also saw that when my father became older, and my mother could link up, cooperate with at least one of my sisters, that it got him into a major predicament, he majorly fell back, and as an imperial officer, it got so far that he told me that if this continued he would after 50 years of marriage get a divorce. That means that this absolute dominance and activity and such things were at the time I'm talking about majorly undermined by my mother and my sister (Bühring, Personal Communication, 31.07.2018, p. CIX, line 620).

To my mind, above quotes illustrates that after all my father's definition of masculinity is both not quite as univocal as it appears and that it, despite his denial, was to some degree

\footnotetext{
${ }^{11}$ Conception of masculinity

${ }^{12}$ My father said 'but' which seems grammatically wrong but I believe that the 'but' referred to me asking if his father did not match his conception of masculinity

${ }^{13}$ In the original transcript my father used the word 'kamellen' which is a colloquial word for being in charge
} 
impacted by his experiences in childhood and adolescence even if more by observing role models of masculinity and femininity that in my father's view were rather negative.

According to the literature, hegemonic masculinity needs to continuously be proven and reinstated since it is both, always in danger of being replaced by an alternative and competing concept of masculinity, and since the privileges available to men who are on top of the male hierarchy are hard-fought for (Bourdieu, 2001). That two of the participants, Dr Lars Bühring and Prof. Dr Schotten explicitly mentioned activity, and performance orientation in their definition of masculinity suggests that they might indeed feel the pressure to incessantly validate and defend their dominance and superiority against other men and perhaps to a lesser degree against women by outstanding achievements and by generally outperforming them (Bourdieu, 2001; Chivers, 2011; Connell, 2005; Coston \& Kimmel, 2012). Although GB and Burkard Rosenfeld did not include being active and performance-oriented in their definitions, it is striking that Burkhard Rosenfeld, just like Prof. Dr Schotten and Dr Lars Bühring, has always been a very active sportsman - something whose competitive character is in the literature highlighted as an early training camp for claiming male hegemony and ensuring and maintaining dominance (Baur \& Luedtke, 2008; Phoenix \& Griffin, 2015; Wörsching, 1999). In contrast, GB has never been overly sportive but did nevertheless make it a point to emphasise his superiority in other areas such as being a successful member of a choir.

Whether ambition and goal orientation is something mostly linked to being a man and desiring to be on top of the male hierarchy, or if this is simply a characteristic of the meritocracy which has developed as a result of the increasing neoliberal socio-cultural and political-economic orientation in the west, cannot be conclusively answered. There can, however, be no doubt that a majority of individuals who grew up and now live in neoliberal societies, regardless of sex or sexual orientation, feel that one's value is crucially dependent on what one represents, has achieved and on the social status one can thus claim (Bourdieu, December 1998; Chomsky, 1999; Harvey, 2007a).

Social recognition and linked status are in neoliberal meritocracy majorly determined by the professional position one has. Since as discussed in chapter 7.1 my interviewees in many ways matched the criteria hegemonic masculinity is characterised by, I assumed that their career progression and professional position would play a crucial role in the identity construction of the participants (Cornwall et al., 2016; Gill, 2008; Mac an Ghaill, 2003). To some degree the answers of my interviewees confirmed this assumption but, and somehow to my surprise, the ambiguity evident in each individual interview and across the interviews with regards to gender differences and the construction of their own masculinity also surfaced when the questions focussed on their career progression. 
My interviewees' account of their professional development constituted a major part of the entire interview. Judging from their enthusiasm, all interviewees seemed to have, understandably given their career development, enjoyed telling me about the different steps of their career, the challenges they had met and the experiences they had made. Nonetheless, only Prof. Dr Schotten admitted that he valued the social recognition and status linked to his profession: "Yes, it does have an influence on me. That is stabilising for the self-confidence," (Schotten, Personal Communication, 18.07.2017, p. LVIII, line 7-8). A little later, he first conceded when I suggested that it might have been nice to spend some more time with his family but then ended his comment with the following words: “...Yes, there is, let's say, the male gene, performance orientation. That one I don't want to say explicitly seeks but appreciates if one receives social recognition from the outside...," (Schotten, Personal Communication, 18.07.2017, p. LIX, line 7-10).

When I asked GB if his job had an influence on how he viewed himself, he did not answer the question and instead talked about his career development and the challenges he had met (GB, Personal Communication, 15.12.2017, p. LXXXVII, line 7-12, p. LXXXVIII, line 1$36 \&$ p. LXXXIX, line 1-14). The issue of one's need for social recognition and appreciation as linked to self-worth only came up when we discussed his retirement (which will in detail be discussed in 6.2.3) when he said: “...Well, yesterday, let's say you had a bag full of appointments and tasks to be done ${ }^{14}$, and you were very important. And today nobody really gives a damn. What you do and how you do it," (GB, Personal Communication, 15.12. 2017, p. XCIV, line 18-20).

Both, Dr Lars Bühring and Burkard Rosenfeld denied that the prestige linked to their high position in the corporate world had any significance for them. Burkhard Rosenfeld said: "I always have or I love, I love discretion and I don't like public appearance. And that is why many people did not even know what I was doing...," (Rosenfeld, Personal Communication, 17.07.2017, p. XXXII, line 1-2) When I asked my father if he believed that his prestigious position in a well-known company in Cologne had an influence on how outsiders perceived and evaluated him, he agreed with this but when I then asked if he liked or disliked that he said: "I understand that somehow but really I found it neither pleasant nor unpleasant but it was simply aware of it. That was the way it was. I can't defend myself against it, but I also don’t find it particularly great," (Bühring, Personal Communication, 31.07.2018, p. CCIII, line 4-6).

Interestingly, however, three of the participants, namely Prof. Dr Schotten, Burkard Rosenfeld and GB, explicitly reflected on the downside of making a career and, in fact, in

${ }^{14}$,bag full of appointments ' is a colloquial expression for having a packed agenda 
hindsight, all of them questioned if they had set adequate priorities during their career. Burkard Rosenfeld said within this context: "I neglected my family. I neglected my family; I did not willingly neglect my family. I didn't work at weekends except when I travelled. But at weekends I had to do sports. And if my wife didn't join me in it, at that time it was tennis; then it is her own fault...the family got a raw deal according to current standards,"(Rosenfeld, Personal Communication, 17.07.2017, p. XXXII, line 19-21 \& p. XXXIII, line 1-2 \& line 20-21). When I followed this comment up by asking: "Current standards?" he replied: "No, according to general standards," (Rosenfeld, Personal Communication, 17.07.2017, p. XXXIV, line 1).

Prof Dr Schotten commented on his priorities during his working life as follows: "My private life was structured so that I worked too much...my wife always says that she was a single mother. There is undoubtedly some truth in this. I worked a lot, a lot. But I also...We always went on holidays together with the children," (Schotten, Personal Communication, 18.07.2017, p. LVIII, line 11-12 \& line 13-15). Shortly after, he added: “Also at weekends. Yes, yes, and many evenings, yes, yes. Frequently. And then I was away a lot for public lectures or something. That has (sighs), when thinking back, maybe been the wrong evaluation. That one put too much value in it," (Schotten, Personal Communication, 18.07.2017, p. LIX, line 1-4).

GB first said when I asked him how he had managed all his professional obligations: “...For me, there really just was the job and then at the weekend family. I had no hobbies, for hobbies I had no time. Since during the week my job was really open end, I kept the weekend free deliberately to spend time with the children,"(GB, Personal Communication, 15.12.2017, p. LXXXIII, line 4-7). Within another context, he then said:

As successful as I was with what I was doing, I also had to sacrifice a lot for that. Well, regarding my family, that was ...You see, the fact that I separated from B., that we separated ... That was, let's say, I used to say this quite tomboyish: I sacrificed my first marriage on the altar of my career. ... Today I would think about this (GB, Personal Communication, 15.12.2017, p. XCII, line 13-15, line 16-17 \& line 28-29).

My father did neither in the interview nor in other conversations we had ever indicate that he felt that he had spent too little time with his family because of his job. However, his personal situation was also entirely different from the other participants'. By the time my father's career really sped up, he had already been divorced from my mother for some time, I lived with her and only saw my father at weekends and in his rather unconventional new relationship he and his partner did not live together and made it a point to both live their own lives rather independently from each other. I, indeed, never felt that my father did not have enough time for me since we saw each other regularly and also spent holidays together, sometimes just us two, and sometimes with his new extended family. As far as I can recall, 
my father never revoked any appointments we had made for job-related reasons. In fact, he also stressed in the interview that one of the advantages in his last job was that:

[...] without needing to talk about it explicitly we all agreed that during the week, what I mean, from Monday to Friday, there was practically no time limit for our activities, so we did not have a time card or punch card in the back of our minds. But we agreed, all, all three and also just executed this, that the weekend is a private, a private thing, that essentially two days of the week are off....Another thing was that we all took holidays in an adequate, more than adequate time frame...(Bühring, Personal Communication, 31.07.2018, p. CXIII, line 10-16 \& line 19-20).

When the interview focussed on the career of the participants, I asked them to tell me what they had particularly liked and disliked in their respective jobs. Interestingly, not only were all answers in essence somewhat similar but they also a revealed an understanding of masculinity which contradicts the current hegemonic understanding of transnational masculinity and resembles a somewhat old-fashioned conception of being a man (Alsemgeest, 2014; Connell, 2009; Kimmel, 2001; Needleman, 1994). Dr Lars Bühring, Burkard Rosenfeld, GB and Prof. Dr Schotten all agreed in their dislike for multinational companies and/or the practices and behaviour transmitted as a prerequisite for success in an international (corporate) environment. Dr Lars Bühring said when I asked him why he resigned his job at Esso:

[...] since this environment of big corporations did in many ways not match my conceptions.... This absolute totally impersonal relationship between activity and people, hierarchies and regulations absolute totally determined the relationship with each other and didn't allow for individual characteristics or something like that. So that he could not at all identify himself with this activity, which he did (Bühring, Personal Communication, 31.07.2018, p. CX, p. 21-22 \& p. CXI, line 19-23).

At another point in the interview, my father remarked that he would not ever want to go back to working in his former company. When I asked him why this was the case, he replied:

[...] because the way that the business is conducted at the moment does not correspond with me. That is something totally different, possibly not less successful, but it is something which does not correspond with me and what I wouldn't want to do...That means the interest in the activities in the company, in the companies where something which counts is happening, where in the end the money is made and where the future lies, that was abandoned, and it was replaced by an ever-increasing bureaucracy and control... That means that on the quiet, a top-heavy bureaucracy has developed and the activities of the board of management at Indus concentrates on other things that do not find my interes. (Bühring, Personal Communication, 31.07.2018, p. CXVII, line 4-7, line $14-18$ \& line 21-23).

Burkard Rosenfeld replied when I asked him why he did not like big corporations: "I am not a politician. I am constructed rectangularly. That means I can only, I can only do truth or not do it. I love, or I hate underhandedness und all that can be found in the daily business of big 
corporations...this is the culture of multinationals,"(Rosenfeld, Personal Communication, 17.07.2017, p. XXIX, line 11-13).

GB remarked about his experiences as a top manager in a multinational company:

I, of course, earned the most money as a top manager, only that then you play in a totally different league. There it all revolves around politics, diplomacy, coordination, evaluating and that is then, let's say, sometimes tiring since you can hardly decide anything by yourself....I could have perhaps reached more if I had sometimes sold my own grandmother. I never did that. In the end, the human being was always more important to me than the job (GB, Personal Communication, 15.12.2017, p. LXXXIV, line 14-18 \& line 21-23).

Prof. Dr Schotten had never worked for a big company, but when we discussed his job choice and the option of becoming a diplomate, he repeatedly said: "I'm not a diplomatic type...I am not diplomatic. No, not at all. ... No, not a diplomatic type. I am totally undiplomatic. Totally undiplomatic," (Schotten, Personal Communication, p. LIV, line 12 \& line 14-15). Sometime later, when we talked about his activities in the chamber of notaries, he remarked:

The chamber is politics. I gave it up after I had done it intensively for eight years since there was too much politics. You know, they said this today, tomorrow something else and the day after that something else, that all was not consistent and... You decide something and then a day after nobody knows what had been decided the previous week (Schotten, Personal Communication, 17.07.2017, p. LXII, line 3-7).

Within this context, I also enquired about what the participants found most important in their professional environment and what was most essential with regards to their job. Rather surprisingly given their individual differences, all interviewees' answers centred around very similar aspects.

Prof. Dr Schotten first replied to my question if he had ever regretted his decision to become a notary: "No, not at all. For me to become a notary was absolutely right. Absolutely right," (Schotten, Personal Communication, 18.07.2017, p. LVII, line 18-19). When I enquired whether his job had influenced his self-perception, he said:

Yes, to a very high degree. To a very high degree, I would say, since I believe I have chosen the right profession for me. That, that was a job in which you can work very intensely calmly, rationally. Also balanced and with crucial problems and that, I believe, influenced my life (Schotten, Personal Communication, 18.07.2017, p. LVIII, line 1-4).

At other points during the interview, he reflected on the reasons for enjoying his job:

What I enjoyed in being a notary, that as a notary, you have a lot to do with people who come to you with crucial problems...Y You deal with the central problems of people...Y You really have to do with the essential references of human beings. ...And of utmost importance was of course also that the people had trust in you and I believe I received that to a relatively high degree (Schotten, Personal Communication, 18.07.2017, p. LVI, line 15-16, line 19-20 \& line 22-23, p. LVII, line 13-15).

When GB talked about which job he had enjoyed and why he enjoyed it, he said: 
Well, listen, I enjoyed travelling and issues which involved making decisions. I have always said my best time; my happiest time was when I was central purchaser since there was nobody who in any way lay down the law to me as long as I was successful in what I was doing and had decided, I had all the freedom of the world (GB, Personal Communication, 15.12.2017, p. LXXXIV, line 7-11).

Shortly after he described his work ethics:

[...] nobody of these people knows with how much turnover I had left the Metro, how many countries I established in my job as a fully authorised representative. Nobody knows that. But that I was somebody who was open and fair, whom people liked working with, with whom they didn't get the impression, that, let's say, that they were only pressured and beat, or let's say, wrongly accused, that was really what I stood for. I am convinced that I could have climbed the career ladder higher, had I, let's say, been an 'ass', well and had tried to profit at someone else's expense. That wasn't worth it for me. That wasn't worth it for me. Well, I always wanted to look at myself in the mirror in the morning and be able to tell myself that I could look the people I worked with straight in the eye (GB, Personal Communication, 15.12.2017, p. LXXXVI, line 19-30).

At another point in the interview, he reflected on the significance of staying true to yourself in whatever position you are in: "Authenticity has to be there... Somebody can say I view this differently,, but he cannot say; he pretends to be something what he isn't," (GB, Personal Communication, 15.12.2017, p. XCIII, line 18-20).

Within a similar context, Burkard Rosenfeld said:

At Indus, I enjoyed that from the beginning I could make decisions autonomously. At Indus, I enjoyed that in areas in which I had little competence and which I also was not that interested I had good conversation partners on whose vote I could count. At Indus, I enjoyed the way we worked together...the corporation was always fair, and there was no case in which I couldn't count, in which I couldn't count on the chairman (Rosenfeld, Personal Communication, 17.07.2017, p. XXX, line 5-9 \& line 12-14).

Shortly after he then said: "I always lived in the certainty that I could count on my colleagues," (Rosenfeld, Personal Communication, 17.07.2017, p. XXXI, line 1-2). And when I asked him if his position played a role in his identity construction Burkard Rosenfeld said:

Well, I identify myself by the fact that employees from as long as 45 years ago contact me to wish me Happy Birthday or to ask for advice or something like that. That motivates me, or it motivates me that a managing director I had dismissed quite at the beginning of my time at Indus, that I maintain nearly friendly contacts with him. It motivates me; I am motivated by the fact that I could stay true to myself...(Rosenfeld, Personal Communication, 17.07.2017, p. XXXII, line 5-10).

Dr Lars Bühring described his work at Indus very similarly:

That what I enjoyed especially there (at Indus) was that I could essentially define the crucial criteria for my actions and the limits of my actions more or less myself and that we, K. and then later Rosenfeld, so the third member of the managing board, got along very well and the basis of that was what I had said previously, that we trusted each 
other totally, that you could trust in what the other did, that it is a ... that the other uses his autonomy responsibly (Bühring, Personal Communication, 31.07.2018, p. CXII, line 4-10).

A little later, my father replied to my question if there was a necessity for hierarchies:

There were practically no hierarchies, also in the relationship to the companies and the management board responsible for them the hierarchy was... or let's say, that hinting at the hierarchical rank was not really an argument in the relationship, but the argument was always persuasion of something or else to think about something together and to find a solution together and to not have a preconceived schema from the start (Bühring, Personal Communication, 31.07.2018, p. CXII, line 12-18).

The above quotes of my participants are an expression of a value system which centres around notions of straightforwardness, fairness, authenticity and integrity whilst disapproving hierarchical structures, underhandedness, gaining profit on the expense of others and greed - aspects which characterise today's practices in the corporate world (Bourdieu, December 1998; Chomsky, 1999; Cornwall et al., 2016). One could, therefore, say that the interviewees represent a dying out species of men in today's harsh, competitive and unforgiving environment of neoliberal greed for profit and social recognition. Much like the protagonists in The Expendables trilogy the interviewees' find meaning and motivation in and through their occupation but not by aiming for generating the highest profit and becoming more powerful by all possible means and without consideration for others (compare chapter 5.1-5.4). Instead, they emphasised the importance of staying true to yourself by treating people fairly and sensibly and by using argumentation rather than authoritarian force to lead people. At the same time, staying and being in control and having the freedom to make autonomous decisions, aspects which play a crucial role in the current hegemonic construction of corporate masculinity (Baur \& Luedtke, 2008; Collinson, 2010; Nilsson et al., 2013), also transpired as representing a critical element for the interviewees' positive evaluation of their professional position.

In my view, the participants' emphasis on personal integrity, on treating people fairly and on acting morally correct whilst determining individual autonomy and control as a prerequisite for job satisfaction and intrinsic motivation in many ways matches the construction of masculinity in The Expendables trilogy. Although muscularity did not seem to represent something which the interviewees viewed as essential criteria for their understanding of masculinity, they all made it a point to stress their physical fitness, and for three of the participants, Burkard Rosenfeld, Prof. Dr Schotten and Dr Lars Bühring doing sports regularly and at a more less high level has clearly been an essential part of their lives and, thus, of their identity construction (Rosenfeld, Personal Communication, 17.07.2017, Schotten, Personal Communication, 18.07.2017, GB, Personal Communication, 15.12.2017, 
Bühring, Personal Communication, 31.07.2018). At the same time and similarly to the narrative contextualisation of the protagonists in the three Expendables films, it disseminated that activity, autonomy, a meaningful occupation and moral superiority had been crucial aspects in the professional contexts of my interviewees. As such the participants' construction of their male identity combines, as I had assumed, socio-cultural narratives of nowadays marginalised conceptions of masculinity with current hegemonic understandings of masculinity (Connell, 2005; Coston \& Kimmel, 2012; Hall, 2002).

As discussed in chapter 4.2, the breadwinner model of masculinity is characterised by clearly divided gender roles in which the woman stays at home and takes care of the children and the household whilst the man provides for his family by performing a meaningful occupation in the public sphere (Gray, 2000). All the participants in some way referred to this rather old-fashioned masculinity ideal popular in the $19^{\text {th }}$ and early $20^{\text {th }}$ century (Gardiner, 2004; Kessel, 2003). Simultaneously, the strong emphasis the interviewees placed on activity, performance, personal autonomy and rationality respectively control of oneself are vital aspects in the socio-cultural narratives of modern understandings of hegemonic masculinity (Connell, 2009) evidences that these standards have impacted the participants' identity construction throughout their working life and until now. This could also explain the ambiguity I identified in their perception of women and their position in relation to men.

Although all interviewees expressed their belief in a gendered role division, their relationship to their wives or female colleagues was predominantly not characterised by the hierarchical structure typical for ultra-conservative patriarchy dominant in the $19^{\text {th }}$ and early $20^{\text {th }}$ century (Colvin \& Davies, 2008). Instead, it seemed to me that the participants often viewed women as equal partners, particularly when they displayed characteristics such as being active, rational and autonomous usually linked to masculinity whilst still fulfilling their role as mother and housewife.

This could be an outcome of the loosening of gender hierarchies as a result of civil rights movements and feminism in the $70 \mathrm{~s}$, but it certainly is also the result of the increasing influence of neoliberal meritocracy in which outstanding achievement can override social marginalisation caused by gender, sexual orientation or race (Baur \& Luedtke, 2008; Duggan, 2002). Lastly, establishing integrity, authenticity and fairness as critical constituents of meaningful professional activity is based on a nowadays marginalised conception of masculinity firmly linked to working-class men (Banet-Weiser, 2014; Hall, 2002, 2015; Meuser, 2016). As such, one could say that the participants utilised and combined masculinity conceptions, hegemonic at different times in their lives, to form a consistent identity expressed in their individual life-course narratives. 


\subsection{Reflections on Ageing, Masculinity \& Control}

Older people in western socio-cultural settings are often viewed and treated as an ungendered category whose chronological age overwrites any individual characteristics and intersectional differences such as race, sexual orientation, gender, able-bodiedness, social class and educational background (Cruikshank, 2013). By contrast, the lived diversities of old age are impressively illustrated by my interviewees' accounts; suggesting that the lifecourse narratives and identity constructions of people in older age are possibly more varied than in any other life stage (Baars, 2013; Gullette Morganroth, 2004). Being side-lined and the relative flexibility of the cultural frame of successful ageing defining it as basically not giving in to ageing as decline leave older people in an ambiguous vacuum in which they, on the one hand, are expected to stay active, productive, autonomous and youthful, whilst, on the other hand, there are no clear socio-cultural narratives of how to achieve this. It is left to every older person to interpret and fill the rather vague and broad socio-cultural frames available for constructing an older identity on the basis of their individual circumstances, experiences and worldviews (Segal, 2014; Spector-Mersel, 2006).

Nonetheless, as mentioned throughout my thesis, the gender hierarchies constitutive for earlier stages of life continue into older age (Chivers, 2011; Dolan, 2018; Richardson, 2019). Even in older age, men can retain certain aspects of power such as financial stability or wealth, especially if they are white and heterosexual. Being a man still provides them with privileged status. At the same time, the natural process of ageing sooner or later affects elements crucially tied to socio-cultural understandings of hegemonic masculinity in the west. Physical strength and endurance, mental agility, memory as well as sexual potency will eventually decrease and being a retiree means having lost a crucial means of male identification and social status and power, namely one's professional occupation. "Thus, in many ageing men's lives, there is a strange, contradictory mixture of some legacies of masculine and heterosexual privilege and power interacting with some reduction and loss in their social and bodily power," (Jackson, 2016, p. 10-11). How older German men handle these ambiguities and how they use and fill the available socio-cultural frames of successful ageing and ageing as decline to construct their identity in later life was and is the focus of my research.

Given the high relevance one's profession and linked status in men's lives, I had assumed that retirement would represent a crucial transition in my interviewees' life courses - one which would be quite challenging and potentially traumatising. In fact and to my surprise all interviewees, except one, described their experiences with retirement predominantly positively. Prof. Dr Schotten said when I asked him how he had experienced the transition 
from employment to retirement: "The transition was for me ... My son is a psychiatrist, and he said to me that I had managed it astonishingly well. I managed it astonishingly well. I, of course, still had ... First of all, I have many hobbies, let's say. Running. Then philosophy. I do quite a lot of philosophy," (Schotten, Personal Communication, 18.07.2017, p. LXI, line 5-8).

Burkard Rosenfeld said when I asked him how he had found it to be suddenly a retiree:

That was wonderful. I was wonderful. I had just felt that the backpack ${ }^{15}$ had become too heavy, had I continued a little longer in this way then it could have been that I would have in some way collapsed. I enjoyed it. I had not planned anything. I did therefore not do the things typical retirees do, travelling around the world, writing a book, study, walk around the house, all this I didn't do. I slept in. And then I read books... Before I could not ever read a book in one stretch. I really enormously enjoyed it. My wife was really surprised; he doesn't do anything (Rosenfeld, Personal Communication, 17.07.2017, p. XXXVI, line 4-12).

In reply to my question of how my father had found it to be a retiree, he said:

Great because, I have to say that, as great as the entire time was, previously the weeks had professionally been rather tense so that the evenings, at least during the week, did not offer themselves for other major activities or major, let's say, mental efforts were out of the question. So that one thing, to my mind, was neglected massively and that was reading. And I enjoyed it immensely to have the possibility to choose and structure my activities from morning till evening autonomously. And that $I$ in the beginning, in the beginning, I, let's say in the first three to four years or so, I read a lot and everything from fiction to non-fiction but predominantly books with a focus on historical developments...in addition, what we did... what we, of course, did not do so much since holiday times were filled with, well, content of a sportive character, at least in winter, we additionally undertook different journeys. To Egypt and to Russia and to Italy, Spain and France anyways and also to Canada (Bühring, Personal Communication, 31.07.2018, p. CXV, line 1-16).

However, during the interviews, it turned out that all three men, Prof. Dr Schotten, Burkard Rosenfeld and Dr Lars Bühring, had, in fact, stopped working only shortly before the interview took place or were still professionally active although much less than they used to be. Prof. Dr Schotten had after his retirement as head of his notary office, still continued working for one particular company until 2012 and had revised the Staudinger ${ }^{16}$ in the past year. "As such, I haven't done any legal work for a year," (Schotten, Personal Communication, 18.07.2017, p. LXI, line 1-2). Burkhard Rosenfeld's period of pure retirement had actually only lasted for about one year. In addition to his position as head of the supervisory board of Indus from 2009 to 2014, he was also still actively involved in two other mandates at the time of the interview although he said "I am now considering if I should stop this year or if I should continue as long as I am healthy," (Rosenfeld, Personal Communication, 17.07.2017, p. XXXVII, line 9-10). My father officially retired in 2004 but

\footnotetext{
${ }^{15}$ In German a colloquial expression for burden

16 an influential commentary on the German Civil Code
} 
continued as an advisor for his former company until 2008 and then in 2010 first became a member of the executive board of a private foundation in Germany in which he then took on the position as head of the executive board in 2016 which he holds until today. As such, my father as well as Burkard Rosenfeld and to a lesser degree, Prof. Dr Schotten cannot be viewed as having fully retired. However, compared to the times before they officially retired, their professional involvement has markedly decreased, and therefore all three participants generally perceive themselves as being retired.

When I asked my father if he enjoyed his involvement in the foundation, he said: "That is the way it is quite nice," (Bühring, Personal Communication, 31.07.2018, p. ). He then explained what exactly he enjoyed about his work in this honorary post:

What I enjoy about it is that I see that certain characteristics or abilities that I happen to have, that I can apply them within the frame of this foundation quite effectively and that this foundation, one of the most significant private foundations in Germany, that they, as I found out, very often are led more or less on the level of an allotment association, and that is obviously a shame for, in this case, really big, big assets, that are placed in these foundations, that, that are only partially handled professionally... That therefore what the founder, the founders wanted to achieve is realised less and less and that the resulting trench warfare and with it the waste of energy and also money leads to the foundation not achieving what it could have achieved (Bühring, Personal Communication, 31.07.2018, p. CXIV, line 5-16).

In reply to my question whether Burkard Rosenfeld enjoyed his different current mandates, he said:

Yes, I noticed during the time when I was a retiree, I don't know, maybe it was two years, that my mental sharpness declined, my memory, that I had to more often search for words and facts since I had forgotten them. This being permanently occupied with the matters of these firms as I think about these things sometimes more, sometimes less, that sharpens the senses here (points to the head) as well as in the behaviour. I have learned to be patient, particularly that...the weakness I have. I have learned not to do the things I want to do spontaneously (Rosenfeld, Personal Communication, 17.07.2017, p. XXXVII, line 12-19).

Since Prof. Dr Schotten had actually not been active in his former profession for about a year at the time of the interview, I did not enquire what he enjoyed about his current professional activities but instead asked him immediately if he felt that people viewed him differently since retirement and he replied: "No. Of course, you are challenged in your job. You do something for somebody else. And then you are also rewarded, but that of courses ceases to exist. That is, well, ...These relationships were, of course, essential to me," (Schotten, Personal Communication, 18.07.2017, p. LXIII, line 4-6 ). When I enquired if he missed this feedback and how he had replaced it, Prof. Dr Schotten told me about his close relationship to two female colleagues who frequently sought his advice and finished by saying: "...As such, I am still in the game. But I don't miss it. I don't miss that anymore," (Schotten, 
Personal Communication, 18.07.2017, p. LXIII, line 14-15). When I asked my father if his retirement had changed the way people view him, he said:

I have the impression, especially with outsiders, you can't get rid of this totally. That means you take that with you. You don't ever appear as a new-born pensioner... No, no, not necessarily. No, well a silly example, I have relatively little to do with my previous classmates ...but occasionally somebody turns up whom I did my A-levels with, then it really comes to light that what I used to do professionally really plays a role, well, is an aspect which contributes to his evaluation of mine (Bühring, Personal Communication, 31.07.2018, p. CXV, line 20-22 \& p. CXVI, line 3-4 \& line 5-8).

The above quotes as well as the continuation of the interview, in my view, illustrate clearly that the three participants view themselves as having mastered the transition from working life to retirement quite successfully and welcome the increase in personal autonomy and control of how to fill one's time. This became particularly clear when I asked them if their priorities had changed as a result of being retired. Except for GB, they all enthusiastically agreed, and all mentioned that they now had much more time to spend with their families and friends and to dedicate to their favourite pastimes, namely sports and reading, which in turn had markedly increased their quality of life. Simultaneously and in line with key findings in the literature, it seemed to be of high relevance for these three interviewees, too, despite retirement, stay busy and feel that the activities they fill their lives with are meaningful and contribute, in a closer or broader sense, to society - something which seems to be possible mostly by being part and taking part in the professional context (Carroll \& Bartlett, 2015; Cruikshank, 2013; van Dyk, 2014).

It appears that it is precisely this meaningfulness whose lack GB, the fourth interviewee, misses in his life as a retiree. He first said:

[...] two things were significant for the later development. I had been on the move internationally a lot, and that is why I could not, as one says, take root somewhere... That meant in consequence, I had no big network outside of my job, and I also didn't have some memberships in clubs or hobbies such as tennis or golf or whatever. Where you could say, there are a few people who will also be there, when you have a lot of time as is the case now (GB, Personal Communication, 15.12.2017, p. LXXXII, line 25-26 \& p. LXXXIII, line 1 \& line 7-11).

He then sometime later, in reply to my question of how he had experienced it to from one day to the next wake up as a retiree, said:

Well, really, that was traumatic, let's say. Because you suddenly feel as if you were totally dispensable. ... That is the case till today so that I really say; it is a waste of the time I live without being able to fill it with something, I could say it makes sense... (GB, Personal Communication, 15.12.2017, p. XCIV, line 4-5 \& line 7-9).

When I asked if being retired had an influence on how GB viewed himself, he responded:

Yes, definitely....That you have a problem to do just something. Because I would tell myself, don't now do something just to have an alibi. According to the principle, the main thing is that he is off the road so that I don't get run over by a car. That is 
not enough for me. I would have to do something which fills me with sense. Where I can say, I can move something; I can do something....For me, it would have been more significant to pass on something which is needed. What makes sense. Yes? T. says I should write a book. I don't know (GB, Personal Communication, 15.12.2017, p. XCV, line 3-8 \& line 14-16).

I then enquired if his family could not take that place and GB said:

No. Let me say it this way. How deep that goes, you can see. I am a believer. Not a catholic, in how the church prescribes it, but I am a believer. And since I managed to get over my lung cancer, I often asked myself ... Then I said, God, you must have had a plan. So. And now I really look right and left, very attentively, so that I can identify what God has saved me for. ...But that is somewhat characteristic for the search you are conducting. That is then, ... you then could also say, I am looking for a new centre of my life. Before that were my family and the job. In this combination. And now that is gone... (GB, Personal Communication, 15.12.2017, p. XCV, line 18-21 \& p. XCVI, line 6-8 \& line 9-12).

GB's comments highlight the high relevance he attributes to the meaningfulness of his activities in older age. He insisted that just keeping busy with something to avoid feeling futile would not suffice but that to feel useful the activity needed to contribute to society significantly. As such his remarks reflect hegemonic socio-cultural understandings of successful ageing as well as ageing viewed as decline which are crucially based on neoliberal conceptions of productivity and achievement - a fundamental prerequisite for socio-cultural recognition (Ayalon \& Tesch-Römer, 2018; Carroll \& Bartlett, 2015; Cruikshank, 2013; Fung, 2013; Jolanki, 2009). Although the other participants seem to have found some of this meaningfulness in the intense engagement in sports, reading and travelling as well in the intensified relationships to friends and family, it is striking that all three took on some professional obligations after their retirement. This, in my view, indicates that not only for GB but for all participants family, friends and pastimes could not entirely adequately fill the gap left by retiring from their professional position. This is especially true when additionally considering that GB was the only interviewee explicitly linking a feeling of worth- and uselessness to a lack of meaningful occupation since his retirement. This suggests that somewhat working within a professional, and one might say male, environment bears particular meaning for older male identity (Baur \& Luedtke, 2008; Jackson, 2016).

At the same time, all interviewees explicitly linked attributes commonly alluded to alternative understandings of a good old age which used to be commonly linked to western conceptions of ageing (Woodward, 2003) but which are today hegemonic especially in Asian countries (Fung, 2013; Grossmann et al., 2012), such as wisdom, emotional balance, serenity and mentoring, to their older age. As a result of the increasing hegemony of neoliberal values in the west, this socio-cultural narrative of ageing has mostly been replaced by characteristics better suited for serving the neoliberal agenda, such as activity and productivity, 
consumption, speed and youthfulness (Baars, 2013, 2015). As can be seen in the above quotes, the participants are clearly influenced by these current views of successful ageing whilst, however, also incorporating alternative views of ageing into their self-conception. Burkard Rosenfeld said in reply to my question if his priorities had changed in older age: "Yes, clearly. ...Wasting no time," (Rosenfeld, Personal Communication, 17.07.2017, p. XXXVIII, line 2). I then asked with what he would waste time and Burkard Rosenfeld said: "With meaningless things, with anger, as far as possible...And this includes, for example, placidity," (Rosenfeld, Personal Communication, 17.07.2017, p. XXXVIII, line 4-7). A little later he explained how older age had changed him: “... I am certainly becoming softer. Well, I certainly also have become softer in this phase. A basic toughness, the willingness to fight and to commit yourself and so on and also to strike has remained but mercifulness has won," (Rosenfeld, Personal Communication, 17.07.2017, p. XXXIX, line 4-7). In a totally different context, when I, within the context of enquiring for whom ageing was more difficult, men or women, focussed on a decline in strength and resultantly control and asked if that worried him, he remarked:

No, that doesn't worry me. There are things I have to think about. Which I am, however, handling quite well since I have a certain humbleness that I want to accept things and don't complain or don't want to kick into the long grass. That is the way it is, and now I have to handle it...(Rosenfeld, Personal Communication, 17.07.2017, p. XLVIIi, line 15-16).

Towards the end of the interview when he told me about a film contextualising a fatherdaughter relationship, he described the father's behaviour as: “...[he] behaved very wisely, that, that impresses me, that I like," (Rosenfeld, Personal Communication, 17.07.2017, p. XLVII, line 9-12).

When I asked my father to define what older age means to him, he said:

Older age, first of all, means to me that you are not in a hurry anymore. And to not being in a hurry anymore means that you are possibly in your reactions not anymore, that the amplitudes in his actions and reactions cannot deflect up and down so much. That, therefore, means that with reference to older age total delight but also total despair do not happen as such anymore (Bühring, Personal Communication, 31.07.2018, p. CXVIII, line 4-9).

In reply to my follow-up question asking if this referred to tranquillity he then said: “Absolutely, well, I don't want to say, I refrained from saying, but now I'll say it anyway, in the direction of wisdom, but something like that...,"(Bühring, Personal Communication, 31.07.2018, p. CXVIII, line 11-12). My father further elaborated on the theme of older age and its effect on him when I asked him if it had any influence on him not to be employed anymore:

No, I can only say that I accept the position I have at the moment or what characterises life at the moment. But if the question aims at finding out if I feel less valuable or 
something like that. Then I have to say that is not the case. Each period has its function. Each period also has its particularities and its beauty and this one, well, I generally find wonderful ..."(Bühring, Personal Communication, 31.07.2018, p. CXVI, line 1318).

Prof. Dr Schotten insisted that his world view had not changed in older age but then admitted: $\ldots{ }^{17}$ Has certainly also led to essential insights. Well, that to perceive what is essential as essential. And that you also ask yourself why you got angry about some things in previous times...," (Schotten, Personal Communication, 18.07.2017, p. LXXI, line 2-4). I then asked him what he considers as essential, and he replied: "Essential for me is a certain inner tranquillity with a philosophical basis...," (Schotten, Personal Communication, 18.07.2017, p. LXXI, line 7-8).

Within this context, it is noteworthy that both Prof. Dr Schotten and GB used to, in addition to their primary jobs, lecture at universities and vocational colleges. Both claimed that they enjoyed working with young people immensely, were highly successful in transmitting their professional knowledge and their experience to and very popular with the student body. Prof Dr Schotten said: "Yes, I really enjoyed that. That was the first time that I noticed that I had pedagogical abilities. And that had sound success...,"( Schotten Personal Communication, 18.07.2017, p. LVI, line 7-9). Shortly before that he had explained to me what university programme he had taught on entailed, and finished by saying:

And then that was really good. I also had a lot of success. A really well-known professor... once passed by the lecture hall and then his assistant said: 'If you want to see a full lecture hall, then have a look in here.' There were in total 200 students. That is quite a lot. ..."( Schotten Personal Communication, 18.07.2017, p. LV, line 25-29).

GB first remarked:

Because much later, as a parvenu top manager, that was for me the most delightful experience that I could pass on my experience to younger people. Since I experienced when I was a young person, what it means when one, who nearly stands on a pedestal in front of you, turns towards you and helps you like a father (GB, Personal Communication, 15.12.2017, p. LXXXII, line 2-6).

Shortly before that he had said about his work at St. Gallen University:

... So. And then the parents are far away, the friends are far away and, well, then there was B., whom they could always talk to, whom they could discuss problems with which they possibly didn't want to discuss with their parents or something like that. And that I enjoyed immensely...The professor of the HSE...always said...:'The problem we have is that by now we have a B-scale since you are by far always the one with most first choices'..." (GB, Personal Communication, 15.12.2017, p. LXXXIX, line 20-23)

I then commented said: "Yes, mentor - you match that a little bit..." and GB agreed: "Yes, mentor," (GB, Personal Communication, 15.12.2017, p. XC, line 2).

${ }^{17}$ Older age 
Although these quotes illuminate and will be discussed in detail in the respective section my interviewees' view on intergenerational relationships, they also fit very well into the context of above-mentioned socio-cultural narrative of ageing linking higher age to wisdom accumulated by and the natural outcome of more life experience and to a mentoring position in which it is the job of the older, particularly male, generation is to pass this knowledge on to the next generations (Ayalon \& Tesch-Römer, 2018; Cruikshank, 2013; Segal, 2013; Wilson, 2000). That the interviewees specifically mentioned how successful and well-liked they were as lecturers and that they enjoyed passing on their knowledge and experience to younger people, however, also points to a fascinating commixture of marginalised and outoff-fashion views of ageing with a neoliberal emphasis on productivity, efficiency and on contributing to society in the life-course narratives of my interviewees (Jackson, 2016; Woodward, 2003).

The theme of efficiency, respectively control recurred several times in the continuation of the interviews. After having asked about how the participants had experienced the transition from working life to retirement, I enquired more about how older age had impacted the lives and identity construction of the interviewees. When I asked if older age had in any way limited their capabilities, all participants conceded to feeling some but minor signs of physical and mental decline. Prof. Dr Schotten said:

I have been noticing that uphill I am still really good, but downhill I am feeling a little bit insecure. That is, therefore, the first limitation, that I...and also the memory declines. That is without a doubt, so the memory is declining...but very moderately and peu-à-peu only. Well, I would say so far I don't feel any limitations due to older age.... But very moderately and only peu a peu...(Schotten, Personal Communication, 18.07.2017, p. LXV, line 2-4 \& line 6-7).

Burkard Rosenfeld remarked in a similar context: "I have noticed that my health, that the stability of my health is declining. I have noticed that my hearing has worsened, that my eyesight has worsened," (Rosenfeld, Personal Communication, 17.07.2017, p. XXXIX, line 2-4). Shortly after he added: "I experience it as a restraint that when I visit the doctor once a year, that at the end of the visit, when you don't hear anything bad, it is experienced like an acquittal. In former times that was a given," (Rosenfeld, Personal Communication, 17.07.2017, p. XL, line 1-3). My father admitted:

That the years take its toll on physical performance and also, let's say, the memory, that is no question...Yes, it is sometimes annoying when you simply forget something or not... Then that is annoying, but it's not as if I then want to kick myself out of anger. That is not the case. But I need to accept this how it is (Bühring, Personal Communication, 31.07.2018, p.CXX, line 3-4 \& line 13-16).

GB first said in reply to the question if he felt old that he did not feel old but then acknowledged: “...well, I sometimes say that some brain cells, let's say, were destroyed 
during chemotherapy but besides that, let's say, I am in full command of my senses. Also physically... No I have no problems with, ...I don't have dementia or something like that...," (GB, Personal Communication, 15.12.2017, p. XCVII, line 1-4 \& line 5-6).

It becomes clear that, although all participants feel that older age has slightly impeded their physical and mental abilities overall they not only perceive themselves as younger than their chronological age would suggest but that they also do not experience the age-related impediments as limiting their efficiency, their autonomy or their masculinity. Burkard Rosenfeld denied that getting older had in any way impaired his manliness while my father said when I asked him if his conception of masculinity had changed as a result of older age:

That is a really difficult question. I think that I especially with regards to this aspect of, not activity, but with regard to the aspect of leading or dictating, that that has led to me taking back its significance a little. That is the crucial point which is linked to something else that I don't believe that what I think and what I believe should happen is written in stone but that there are different views and that other people who see things differently can also be happy and content (Bühring, Personal Communication, 31.07.2018, p. CXXII, line 11-18).

I then summarised his comment by saying that he had become more tolerant and flexible with age, and he replied:

I don't know if I am more tolerant, but I don't react as quickly and suddenly to some things. Possibly as little tolerant as I used to be but with regards to certain things I can listen to much more than that was the case in previous times (Bühring, Personal Communication, 31.07.2018, p. CXXIII, line 2-5).

Although Prof. Dr Schotten denied that older age had affected his identity, his reply resembled that of my father:

No, I don't think so. I am calmer because you can concentrate on essential questions. I am more unconcerned about many things. In law, in politics. Even in politics, many things leave me more unconcerned. As such, I wouldn't say that something has changed (Schotten, Personal Communication, 18.07.2017, p. LXII, line 15-18).

Three participants further underlined and objectified this personal evaluation of their mental and physical status quo by either referring to remarks by others or by explicitly comparing themselves to people of varying ages. My father, for instance, said in response to my question of how old he felt to be first:

... But with reference to feeling it is for me always a little bit the reflection of how others see me. And when I consider, when I consider that to...two young men, who are in their mid-forties and they...that, they are really interested in continuing to play tennis with me, once a week. And that these meetings once a week clearly have a certain significance to them, then I have to say, then I am possibly, also in their eyes and in mine, not quite as old as the chronological age seems to indicate (Bühring, Personal Communication, 31.07.2018, p. CXIX, line 1-9). 
When I followed this up by enquiring if he could pinpoint of how many years he roughly felt younger than his chronological age suggested, he replied:

When I look at my environment, and when I look at the others who are similar to me who clearly have to do with different parts of their body and who also have many bodily deficits, then I believe it to be in the region of ten years, that is I think no... it is something in that range... (Bühring, Personal Communication, 31.07.2018, p. CXIX, line 13-18).

Within this context, I asked him as the only interviewee if he felt that he matched the sociocultural frames of successful ageing which he affirmed and then explained: "Yes because I do appreciate yesterday and also think about it and also bring it up once and again but that I also have many experiences that define the now...I also live now and not only in the past," (Bühring, Personal Communication, 31.07.2018, p. CXXI, line 8-10).

GB first described his parents in law as:

They both sit; they are both 81; nothing happens there. They both sit in front of the TV and watch their soaps. He sits in front of the TV and watches sports. So. And, I mean, they have been married for 65 years, there are not many new things to exchange (GB, Personal Communication, 15.12.2017, p. CIII, line 26 \& p. CIV, line 1-3).

Shortly after he denied being a "typical pensioner" and when I asked him what a typical pensioner is, he answered:

The typical pensioner for me is somewhat the one for whom everything is sorted. Who knows that he can manage with the money he has. He paid off his little house, or a pays a manageable rent. And he can, whatever, go on holiday for a few days. And he has his TV, his sports, his news or something like that. So a regular, manageable routine. And also no high ambitions and expectations. That's what defines the typical pensioner for me, and that is not how I see myself. And I also often see that the typical pensioner has a big distance to the younger generation. There is a gap. And that one I also don't see as applicable to myself (GB, Personal Communication, 15.12.2017, p. $\mathrm{CV}$, line 4-12).

Prof. Dr Schotten did not explicitly compare himself to people of different ages but at some point said: "My wife is healthy. I am healthy. We travel a lot. We also have the ability to...People usually think that we both are considerably younger than we are," (Schotten, Personal Communication, 18.07.2017, p. LXIV, line 17-19). Burkard Rosenfeld did not compare his own or his wife's ageing process with other people's, but he did comment insightfully on the difficulties in the relationship between younger and older people:

Younger people are in a completely different life situation; they have totally different aims and problems than older people. Older people have the tendency to brag with their experience and always think they are right; they want to be right. And that leads to conflicts, some open and some only hidden. Well, if I'll take myself, well, I can't really judge. It is probably like this...The dominance that one represents, even if one doesn't do this actively, limits the freedom and ease of others... You want to be on the 
same level, but you are not on the same level (Rosenfeld, Personal Communication, 17.07.2017, p. LI, line 12-19 \& p. LII, line 2-3).

Burkard Rosenfeld was the only participant who did not use social comparison to others (Corcoran et al., 2011; Festinger, 1954) to underline that he had handled the ageing process particularly well and kept comparably youthful in behaviour and mind. Nonetheless, he, like my father and Prof. Dr Schotten, clearly perceived himself as successfully ageing. This became clear latest when he, as described above in section 6.2., from a variety of images of the media representation of successful ageing or ageing as decline, chose the pictures of successful ageing as resembling him and his situation best (Rosenfeld, Personal Communication, 17.07.2017, p. XLVI, line 6).

Only GB did not evaluate the life-stage of retirement as entirely positive and viewed himself as somewhat unsuccessfully ageing. He, however, attributed that more to the socio-cultural and individual circumstances than to him personally failing at ageing successfully.

The only problem I have is that I, therefore, with my power, my mental and physical power not only feel totally under-challenged but that I do not at all feel placed somewhere. You know? I once said somewhat colloquially; I steal God valuable time. As such as that, I could really do more and would also like to do more than I'm factually doing (GB, Personal Communication, 15.12.2017, p. XCVII, line 6-11).

As a result of my own experiences and the literature review I had conducted, I had assumed that personal control, respectively limitations or loss of it would present a crucial element in the male ageing process. As mentioned repeatedly throughout the thesis, although the obsession with keeping control is surely not a male-specific phenomena, maintaining personal autonomy and affiliated attributes of rationality, efficiency and emotion management appears to be an exceptionally essential element of hegemonic understandings of masculinity (Beynon, 2001; Calasanti \& King, 2015; Connell, 2005; Coston \& Kimmel, 2012; Edwards, 2004; Hearn, 2014; Reeser, 2011). Consequently, I wanted to find out more about how my interviewees dealt with present and foreseeable decline in physical and mental control.

Within this context, GB's life and experience deviate in several ways from the life-course narratives of the other participants. GB is not only ten years younger than the other interviewees, but he was also the only participant who had dealt with cancer twice and overcame it at the time of the interview and then had to battle the recurrence of cancer shortly after the interview. In combination with not having had the opportunity to establish a social circle of friends or follow hobbies regularly because of his work commitments and business assignments abroad, his medical history and his younger age have led to a situation which is markedly different from the other interviewees (GB, Personal Communication, 15.12.2017). His concrete experience with a life-threatening illness and linked confrontation with the 
finality of being and his strong belief in God could explain both, the above-cited conviction that there was a deeper meaning in him having overcome cancer twice (respectively three) times and his attitude towards controlling the process of ageing and ultimately death which I found particularly fascinating:

And then I lay in bed. And when you are in hospital, let's say, you doze all day and then can't sleep at night. Then I lay awake...I planned my funeral; I planned who would get what. I chose the music I wanted to have in the chapel, the Church of the Holy Sepulchre... And then there are the attacks when you only cry. I will not see my grandchildren grow up...And then I only said, God, I cannot handle this. That is too much for me. I'll leave it to you. You decide, and whatever you decide I'll accept. Lisa, from this moment on, I had no problem anymore. I had such inner peace; it was incredible. And I was, however, in a state in which I simply said: God, thank you. I know that I...I will not die. You don't yet want me. Yes, well, the professor at some point said, Mr B. $60 \%$ of the recovery is psychological. But my recovery was totally unexplainable to the doctors (GB, Personal Communication, 15.12.2017, p. XCVIII, line 12-25).

As such it seems that GB is able to tolerate and even welcome transferring personal control to a higher entity whilst believing that the mind rules over the body and struggling with accepting living a life without, in his view, a meaningful occupation. That his understanding of meaningfulness is defined by doing something which is publicly visible and therefore enables socio-cultural recognition and respect is likely the result of his long-time employment in a multi-national company and a company culture deeply infused by neoliberal values of efficiency, productivity and success (Eckersley, 2006; Heron, 2008; Leek, 2016).

I asked Prof. Dr Schotten whether he thought that his sound mental and physical condition was the result of his lifestyle, he said:

Yes, yes...we have been living consequently healthy for many years. We hardly ever go to see the doctor and are members of a health insurance in which we haven't given in a doctor's invoice for 22 years. ... We really live vegetarian. And we eat very, very plainly and simply. And consequently and try to live healthily. My wife buys in the health-food shop, very consistently. As such, I believe that it also has something to do with the life-style (Schotten, Personal Communication, 18.07.2017, p. LXV, line 1012 \& line 15-16 \& p. LXVI, line 1-2).

Burkard Rosenfeld said that besides going to a "top doctor" (Rosenfeld, Personal Communication, 17.07.2017, p. XLLII, line 5) once a year:

I do what I can do within limits. So I don't abstain from sugar or alcohol, and I don't smoke or drink spirits. I am also generally sensible, but I don't do, I don't try through some homoeopathy or something to stay healthy, but I trust that, or differently, I am ready to endure what there comes, and so that I know what is coming in time and can influence it, I see the doctor every year (Rosenfeld, Personal Communication, 17.07.2017, p. XLIII, line 9-14). 
When I asked my father if he believed that one could control or stop the physical and mental changes linked to the ageing process, he replied:

Yes, by living as actively as possible...Yes, for example, by having certain expectations or, no, by executing certain activities which demand mental effort and by executing activities at all, own, and not by just reacting to something and, first and foremost, by not, for example, seeing one's realm of experiences in watching TV (Bühring, Personal Communication, 31.07.2018, p. CXXI, line 17 \& p. CXXII, line 2 \& line 4-8).

GB had already uttered several times that he was convinced of the strong link between body and mind and this conviction also became evident in the following quote:

When you have a job... and what was done there, irregular meals, wrong food, no balance. And that is the long-term effect. And that I only understood, very, very late. In older age. That the psyche, or what you in the Chinese medicine call Yin Yang, that that is very, very important. If you don't take care of this, you will, in the long run, get problems in regions you cannot determine beforehand. Because you don't know how your body will react in the long run and in which area. But for me, this totally obvious today (GB, Personal Communication, 15.12.2017, p. XCII, line 1-8).

Although the approaches towards slowing down and influencing the ageing process vary, overall, the above citations illustrate that all interviewees believe that it is possible to exercise control over the process of ageing. Prof. Dr Schotten and Burkard Rosenfeld understand a healthy lifestyle as the key to contain age-related decline whilst my father sees being and staying active particularly, but not only, mentally as crucial and GB perceives inner balance as essential for staying healthy in older age. However, when taking into account what the interviewees said at other points in the interview, it becomes clear that not only my father but all participants viewed keeping mentally active in combination with a healthy and active lifestyle as the prerequisite for keeping age in check. This suggests that the interviewees internalised current hegemonic socio-cultural narratives based on transmitting the high significance of personal autonomy and productivity and carried them forward into older age (Chivers, 2011; Gullette Morganroth, 2004).

However, although public discourse insists on the personal manageability of ageing, the natural process of decline with increasing age puts limits to personal efforts of setting boundaries to the effects of ageing (Katz \& Calasanti, 2014; Katz \& Marshall, 2003). I, therefore, wanted to find out more about my interviewee's views on age-related illness and death.

Prof Dr Schotten and my father both admitted to being afraid of the process of dying. Prof Dr Schotten said:

At the moment I'm not afraid of death at all. I am only afraid of the process of dying. I hope that...I am not in favour of actively assisted suicide; I have to say honestly. Although the differentiation is difficult. But passively assisted suicide I believe to be 
necessary. That means when somebody is in pain, that one provides pain killers even if that could cost that person's life. Yes, because that is then no meaningful life anymore (Schotten, Personal Communication, 17.07.2017, p. LXVIII, line 19-22 \& p. LXIX, line 1-2).

I then shortly after enquired if he was more afraid of pain or of losing autonomy, and Prof. Dr Schotten answered: "Well, you won't then notice the loss of autonomy. When the loss is there... But the loss of autonomy is, of course, problematic," (Schotten, Personal Communication, 17.07.2017, p. LXIX, line 13-14 \& line 15). In reply to my question what worried him about older age, my father said: "Fear of pain - that is really what moves... the continuous. Or, let's say, the process which happens before dying," (Bühring, 31.07.2018, p. CXXIII, line 15-16). When I probed for more details regarding the loss of control, he said: "Yes, all, all that, no. That you are then in a situation that for you, if physically or mentally or both, really is very unpleasant and in which you are not able to influence or even just to express this," (Bühring, 31.07.2018, p. CXXIV, line 2-5). I then wanted to know what would be worse for him, a loss of control over the mind or over the body?

When you imagine that. When you have no control over your body, then you are still aware of this. As such it will have an effect on the mental state, and that is why, I believe, well, if you decline physically, I find that really very bad, I have to say since that automatically also is something really bad for the mind, I cannot bear that (Bühring, 31.07.2018, p. CXXIV, line 9-14).

In response to the same question, Burkard Rosenfeld said: "Especially mentally. Well, if I am mentally entirely gone then in my imagination, it would be totally painless... And if I was physically fit and stupid, it wouldn't concern me much. ... Mental is important, yes. The mental fitness," (Rosenfeld, Personal Communication, 17.07.2017, p. XLII, line 12-13 \& line 15-16 \& p. XLIII, line 2).

In the same context, GB uttered:

Well, it would be worse for me if I was physically limited. Clearly, because I have experienced that factually, not directly, but through my brother, my oldest brother, his best friend, he is now end 70, was a sports teacher, marathon runner. So sportive all his life... He for months now has to, ... he cannot move and needs to be fed. He has to be taken to the toilet; he has to be cleaned, he has to be dressed, he has to be taken to the table in a wheelchair so that he can be fed. If he wants to read the newspaper, someone needs to turn the pages for him. And the man is mentally fully there. And that I imagine being terrible. I can honestly say, I would get, as a precautionary measure, get the tablet. It costs 5000 Euro... If I am mentally gone, I don't care much about what happens to my body. I won't notice that. But if I am still capable of making decisions and know that my body can't carry its weight. And then the quality of life would have decreased so much for me that I would not want to continue living (GB, Personal Communication, 15.12.2017, p. C, line 1-4 \& line 7-13).

To sum up, Prof. Dr Schotten, Dr Lars Bühring and GB viewed the loss of physical autonomy as worse than a detrimental decline in mental health since they all assumed that physical 
limitations would be particularly hard to deal with and would have grim consequences also for their mental fitness whilst a loss of mental abilities would mean that you do not feel or notice what happens to you. In my view, Burkard Rosenfeld did not actually contradict this but instead wanted to emphasise the significance mental fitness has for him. I, in fact, felt that Burkard Rosenfeld had probably not thought about the potential scenarios of illnesses in older age much before and also did not really wish to do so in the interview. This was confirmed when he, after I commented that if he was physically limited would need a lot of help and asked if that would not be a problem for him, he said: "Yes, of course, that all is a problem. Let's see how it is when the time comes." (Rosenfeld, Personal Communication, 17.07.2017, p. XLIII, line 5-6) To me, this was not only an indication that for Burkard Rosenfeld, both physical and mental deterioration was equally worrying but also that he wanted to end this topic and move on which I did.

I had assumed that the religious inclination of the participants would have an influence on how they viewed age since I thought that if you believe in some form of existence after death you would be less scared of the finality and ultimate loss of control death represents. To my surprise, however, if my participants strongly believed in God, or if they somewhat believed in something being there after death or if they thought that what there was after death was not definable since we do not have access to this knowledge made no significant difference in their attitude towards the end of life. Burkard Rosenfeld outright said that he was not afraid of death at all. When I then asked if he was religious, he said: "I'm searching for faith," (Rosenfeld, Personal Communication, 17.07.2017, p. XLIV, line 3). I then probed further, and Burkard Rosenfeld said:

...I am convinced that God exists. With reference to the details...I am a person who is too concrete to really believe. But I have a certain trust in God as a basis...I believe in, in eternal life and that very deeply ...I believe that there is not nothing (Rosenfeld, Personal Communication, 17.07.2017, p. XLIV, line 6-8 \& line 11 \& p. XLV, line 3).

Both my father and Prof. Dr Schotten explicitly said that they were not afraid of death although Prof. Dr Schotten has his doubts regarding religious understandings of life after death and my father does not believe in being able to access any knowledge about what there is after death. When I enquired further Prof. Dr Schotten said:

...I don't want to rule that out totally. I am therefore in the philosophical sense a believer since I believe. That is the belief that there is more than natural mechanisms, let's say...ethics has to appeal to rationality. To the autonomy, that the human is able to act correctly, even though he seldomly does so (Schotten, Personal Communication, 18.07.2017, p. LXVII, line 6-8).

When I asked my father if he thought about death, he outright denied this and then explained: 
Since I think, I think not after death... my depth or breadth of knowledge does not spread beyond death. I cannot say there is nothing else or there is nothing or something like that. I cannot access a for me satisfying cosmos which I can say I can find myself in. That is...it is a closed door (Bühring, Personal Communication, p. CXXV, line 2 \& line 13-17).

Despite the fact that GB had repeatedly emphasised how strongly he believed in God, he was surprisingly the only interviewee who expressed some ambivalence regarding his fear of death:

Well, I have to honestly say yes and no. If only because...Well, let's say you are not one hundred per cent sure. And especially with a medical history like mine then you often think about how that will be. But then you also try to push that away from you a little bit. According to the principle:....so the only reality that is there is here and now... The only thing that is certain is that one day you won't be there anymore...It is the finality that hits and shocks you so deeply... But it is no fear. It is partly trust in what there is to come...It is a grain of fear because insecurity. But also a grain of hope or faith. That is the situation (GB, Personal Communication, 15.12.2017, p. XCIX, line 3-9 \& line 12-19).

In my view a closer inspection of the above citations reveals that how my interviewees felt with regard to their own death was apparently not so much dependent on religious inclination but much rather a result of personal experiences, individualised value systems and lifecourse narratives. It is striking that my father and Prof. Dr Schotten base their understanding of the world predominately on rationality, logical thinking, on maintaining the highest degree of personal autonomy and on avoiding any type of dependency which they also explicitly linked to masculinity. To me, it follows logically that they were also the two participants who most diligently and analytically examined the possibility of life after death and that both came to the conclusion that besides being unlikely, there is no way of knowing what happens after death and as such it is futile to worry about it.

Both men were also particularly critical with reference to institutionalised religion and particularly to Catholicism. My father was of the opinion that all belief systems are mostly an "auxiliary tool which is aimed at channelling and decreasing the human fear, but really it is an instrument of power for some people," (Bühring, Personal Communication, 31.07.2018, p. CXXV, line 6-8). Prof. Dr Schotten questioned the general strategy of belittling human beings in the catholic church. “...The human being is made to look so small. That is a totally wrong approach. And that leads to humans being reduced to someone who is underage," (Schotten, Personal Communication, 18.07.2017, p. LXVIII, line 9-11). Considering the high significance both attach to individual agency and independence it does neither surprise that the two men refuse to transfer any of autonomy to a third party nor that they view the loss of independence as something to be avoided at all costs. 
Burkard Rosenfeld and GB clearly found transferring some of their personal responsibility to a higher being less problematic. GB explicitly uttered that he found it to be a great relief to not having to carry the responsibility for your life all alone (GB, Personal Communication, 15.12.2017, p. XCVIII, line 18-21).

Burkard Rosenfeld was not quite as expressive about easing the burden of personal autonomy by following and believing in religious doctrines but seemed to appreciate the advice and guiding received in sermons. "...last year the parish priest in the neighbouring community gave me, not only me but all, a small note saying placidness... Then I thought, fitting, I can use that," (Rosenfeld, Personal Communication, 17.07.2017, p. XXXVIII, line 6-7 \& line 12-13). As demonstrated here and in the above citations, it appears that both men's construction of their identity is somewhat more permeable and characterised by ambiguity and doubt than that of my father and Prof. Dr Schotten. As such, their life course narratives of older age seem to, to some degree, permit the inclusion of 'unmanly' attributes such as irrationality, emotionality, softness and even a certain amount of dependence.

Nonetheless and despite the fact that each interviewee applies the neoliberal dictate of productivity somewhat differently, it is evident that they all believe that taking charge of their own ageing process is possible and, in fact, an obligation and responsibility every individual has regardless of gender (Bengtson et al., 2005; Carroll \& Bartlett, 2015; Gergen \& Gergen, 2000; Gullette Morganroth, 2011; Katz \& Marshall, 2003; Tulle, 2015). What, in my view, is, however, gender-specific and indicative for the construction of masculinity in older age is their emphasis on physical and mental fitness, efficiency and meaningful activity rather than on appearance as is often the case with constructions of older femininity (Clarke \& Bennett, 2015; Dolan, 2018; Dolan \& Tincknell, 2012). Notably, the definition of meaningfulness which seems to be linked to professional occupation, the high significance attributed to physically and mentally challenging activities and on comparing the own performance with others' seems to me to be particularly representative for male ageing.

All interviewees were from earliest childhood on trained to prove their superiority and right to being top dog in the male hierarchy through competitive games and challenges whilst growing up in a society increasingly dominated by neoliberal values which likely instilled a view of masculinity into them which is based on being active, productive, disciplined, rational and, most importantly, autonomous (Bourdieu, 2001). Their careers in the transnational and male-dominated corporate environment possibly further supported this understanding of masculinity whose main aspects the interviewees, for lack of alternative socio-cultural frames of older masculinity (Spector-Mersel, 2006), use to construct their identity as pensioners and as successful agers (Cornwall et al., 2016; Griffin, 2005; McDonald et al., 2017). However, during the interviews, it was also evident that older age 
had an impact on the self-perception of the participants. GB expressed this most strongly by saying that he felt useless, which he attributed to not having found a meaningful occupation, yet. The other participants were much more positive about the changes they viewed as linked to an older age. All three said that they had become more emotionally balanced and softer respectively more tolerant. In my view, that all interviewees are for their high chronological age exceptionally fit, mentally and physically, and the fact that older age does not seem to limit their abilities markedly has so far simply not made it necessary to majorly adjust their life-course narratives or the constituents their understanding of themselves as men is based on (Calasanti \& King, 2015; de Medeiros \& Black, 2015; Jolanki, 2009; Katz, 2015; Segal, 2014; Wilson, 2000).

Prof. Dr Schotten actually supported this assumption when he said: "If you are not healthy when you have a walking disability, or you are quadriplegic, then the situation looks totally, totally different... a certain financial security is very important...And not everybody can be so active," (Schotten, Personal Communication, 18.07.2017, p. LXXVII, line 7-8). After all, why should you undertake the challenging and gruelling task of redefining yourself when there is no need for this since your current identity construction still fits not only how you view yourself but also current understandings of successful male ageing which ensures you social recognition and acceptance?

\subsection{Reflections on Socio-Cultural Understandings of Older Age \& Masculinity}

As elaborated on previously, this thesis is based on the assumption that personal life course narratives and individual identity are inextricably linked to the hegemonic socio-cultural narratives of any particular time in any respective cultural setting (Foucault, 2000; Foucault et al., 2008; Haraway, 1988). When viewed through the lens of this ontological and epistemological stance, the selected interviewees' life course narratives are particularly fascinating since they were affected by markedly different constructions of masculinity and ageing.

Having grown up in post-war Germany, my interviewees were exposed to masculinity conceptions based on the Prussian ideal of emotional and physical discipline and power, rationality and unquestionable authority through their fathers and other men of critical sociocultural impact, such as teachers (Crouthamel, 2008; Evans, 2015; Jeffords, 1998; Kessel, 2003; Levsen, 2008). Subsequently, they observed and were surely influenced by the deterioration of precisely this masculinity view as a result of the student revolts in the $60 \mathrm{~s}$ and 70 s in Germany. Gender equality and replacing not only old-fashioned and reactionary 
conceptions of an Arian Übermensch masculinity but also the actual removal of former Nazis in key positions of public life were among the declared aims of civil rights movements in Germany (Brown, 2013; Junker, Gassert, Mausbach, \& Morris, 2004).

At the same time, the rebuilding of Germany by the Allies and particularly the U.S., also meant that their socio-cultural value system has increasingly impacted the German culture. Notions of a free market economy as the basis for social, political and economic stability therefore quickly gained ground and became ever more influential in the coming decades especially within the corporate environment which all interviewees were part of (Brandes, 2007; Dobbins, 2003; Dustmann, Fitzenberger, Schönberg, \& Spitz-Oener, 2014; Lazar \& Mauch, 2004). As a result, former constructions of masculinity shifted and gave room to a hegemonic construction of masculinity which is not anymore based on displaying and applying physical power to ensure dominance but in which core neoliberal values such as productivity, efficiency and personal autonomy and responsibility have taken centre stage (Connell, 1998; Connell, 2009; Hall, 2002).

In addition to the alterations of the dominant socio-cultural perception of masculinity, the interviewees also witnessed the emergence of an alternative conception of ageing alongside the traditional and long-time unquestioned view of ageing as decline. The successful ageing paradigm writes these values of neoliberal meritocracy forth into older age and defines success at ageing as taking responsibility for one's ageing by staying active and independent through following a healthy lifestyle, through disciplining and training one's body and mind and through the consumption of goods and services that support productivity and youthfulness (Bolin \& Skogerbø, 2013; de Medeiros, 2016; Featherstone, 1995; Katz \& Calasanti, 2014; Laceulle \& Baars, 2014; Rowe \& Kahn, 1998).

There can be no doubt that all interviewees have integrated a majority of aspects linked to current dominant understandings of masculinity and successful ageing into their life-course narratives. Yet, all participants were very critical in their evaluation of the present sociocultural and politico-economic system. When I asked Prof. Dr Schotten if there was something that worried him about the future - a question intended to elicit his thoughts on his older age and the possibility of mental and bodily decline - he said:

I am worried about the development of the world. The whole world. The world is going up in flames everywhere. And when you think...we have to grandchildren, and when you think, they are now eight and six and a half, when you think of what is coming their way. That worries us, honestly. Since I am very, very sceptical ... Yes, the general problem is, I believe, that the western world has radically exploited the entire rest of the world for centuries. I has exploited the world radically and it can't redeem that. It is not enough to say now we need to do something for Africa when this country has been exploited for centuries... (Schotten, Personal Communication, 17.07.2017, p. LXVI, line 5-9 \& line 12-16). 
In reply to my question how Prof. Dr Schotten would describe the general attitude of society

to older age, he said:

It is not particularly positive. Yes, it is not very positive. You can see that, for example, in the difficulty 50-60-year-old people have to find employment. Yes, I have never understood that. I always had quite old employees, and I was always very happy with them. Well, this obsession with youth, that is applied in many cases, also in many companies, I find, well not for the 60,70 or 80 -year-old people but for the 50, 60-yearold people, I find that problematic. There it becomes, well, ...you are viewed as belonging to the scrap heap quickly. I find that a very bad development. But it's a typically selfish development (Schotten, Personal Communication, 18.07.2017, p. LXXIV, line 12-19).

I then asked him where he thought this connotation came from, he answered:

I believe it is the result of the ever-increasing selfishness in society. ... That is also an expression of selfishness that is expanding quickly ...Yes, productivity has to run so that one can fulfil all of one's selfish wishes...I do believe that an increasing dog-eatdog society has developed. ...Well, consumption, excessive consumption is an expression of selfishness. A sign of that. A symptom (Schotten, Personal Communication, 18.07.2017, p. LXXV, line 3-5 \& line 24-25 \& p. LXXVI, line 2-5 \& line 9-10).

In response to the same question, my father said:

The general attitude towards older age in this country is, to my mind, not characterised by the appreciation of what somebody did in his life, also not by a certain amount of cordiality towards older age but predominantly characterised by an economic way of thinking that, well, older age has to offer little economically or has little importance, little significance. This might now be changing a little when you consider that especially older people are potential or potent consumers, aren't they? But besides that, ....it is more so that they don't contribute anything to the Gross National Product and something like that so that they more or less live on a thinning tolerance level. ... And that brings us to economic aspects. And something as with the Chinese... who or also in African societies that ascribe a high value to older age and view it with respect and in which the advice of older people has value ... That this doesn't play a role in our society also since at the moment it is fashionable to under all circumstances follow the latest trend. That means what is old is, how the name prescribes, old (Bühring, Personal Communication, 31.07.2018, p. CXXIX, line 1-10 \& line 12-18).

Despite everything my father had said before about his positive experiences with ageing, he, at this point in the interview, became quite passionate and when I probed further, he not only shared his rather insightful analysis of neoliberal society with me, but his comments were indicative also for his exceptionally critical view of politico-economic and with it the sociocultural development of Germany and the west, in general. When I asked him where in his opinion, this understanding of ageing was transmitted, he answered:

They are, for example, transmitted in advertising, they are also transmitted in the law. Well, in the social legislation or something like that, there they are also transmitted. They are also transmitted by institutions such as the church or so... That well...At all different levels, it is never transmitted that the older part of society is part of a society 
in which are all equally on the same level. But it is always assumed that older age means a decline in some way and that is why it only conforms to or deserves a special examination. Yes and really the young and active are really the measure. And when that is the measure, then it is clear that the old are in some way devalued (Bühring, Personal Communication, 31.07.2018, p. CXXX, line 1-10).

GB elaborated on this theme by reflecting on changes in the company culture as well as on a societal level:

...But that, this exemplifies the decline of our system. Because, when I, well, see some of these entrepreneurs ...they all die out... Otto Beisheim always said, I mean he was once the richest man in Germany with roughly 10 billion private assets... he was also in Switzerland ... and he had said to me: 'You know Mr B when I have eaten my bread with liver sausage I am also full.' Yes, these are people with the attitude, let's say, for them, it is not decisive for the war if one billion of profit is added every year. They, when they are full, they are full. So. And that...this view, let's say, that as a top manager you really also have a responsibility for the people who are dependent on the company, that very much got lost since they all are evaluated by the analysts. When, when it is a limited liability company, so, they are only evaluated by the shareholders, by, let's say the EBIT increases, by how the share prices developed and the shareholders, in plain German, don't give a damn. The main thing is that they receive a higher dividend. ... Where, where is the understanding of these people that it is not their job in life to take rather ten than eight billion with them in the grave, that the real aim is to leave behind something which is a little, let's say, more sustainable than ten or eight billion (GB, Personal Communication, 15.12.2017, p. LXXXV, line 16-18 \& line 19-22 \& p. LXXXVI, line 1-16).

Probed by my question enquiring about the basis of the socio-cultural understanding of older age in Germany, GB uttered:

...I think that the older generation will feel increasingly worried about their future. The topic of poverty among the elderly, let's say, will increase. That will be intensified by the increasing number of pensioners from the whole demographic development. ... One has to say that a man who worked his whole life and paid social security and who then today receives a pension of twelve or fifteen-hundred Euro, he can hardly live of that. ...I believe that the increasing gap between poor and rich will lead to massive social upheaval... That is for me the greatest risk. Social injustice is the biggest risk for this country (GB, Personal Communication, 15.12.2017, p. CI, line 9-12, line 15-17 \& line 23-24 \& p. CII, line 13-14).

Compared to the other respondents Burkard Rosenfeld was a little less passionate and much briefer in his response to my question where the rather negative attitude to ageing came from in Europe and the US, and he only said: "Since efficiency declines, since the young very early on are trimmed to go to the top and then when you are there, and then there is an old one, he disturbs. That is meritocracy," (Rosenfeld, Personal Communication, 17.07.2017, p. LI, line 1-3). 
The aspect of efficiency and living in a meritocracy also played a significant role in my father's reflection on the differences in socio-cultural understandings of ageing within Europe:

Since here, since it, except perhaps in the US but in no of the big civilised economically potent countries within which Germany takes a leading position, there is such a and so consistent perception of efficiency...in the moment in which this achievement principle has a special significance, an outstanding significance it is clear that he who cannot achieve anymore also in the social or in the perception of the meaningfulness for society obviously doesn't take a leading position (Bühring, Personal Communication, 31.07.2018, p. CXXXI, line 11-19).

As is evident in the above citations all participants not only were very critical and took issue with the current neoliberal orientation of society, economy, legislation and politics but their comments were also, and despite placing the focus on different aspects within this context, particularly insightful and differentiated. What I, however, found interesting, particularly in light of what the participants had said before was the level of frustration and even anger that shone through in their answers. They clearly felt that older people were not appreciated and respected sufficiently in our society. This became even more pronounced when I asked the interviewees if they felt there were cultural differences in the way older people are viewed. As my father in the already quoted citation, Burkard Rosenfeld and GB both reflected on the differences between western and eastern cultures in the perception of older age. Burkard Rosenfeld said:

...In Japan seniority is simply one level in life, the highest level. So an older person there plays a dominant role. With us in Europe that is not the case. In Europe, there is retirement and the old receivers of a pension who take away money from the young or whatever or the old ones that are glued to their jobs, who don't want to let go. That is... for me the biggest difference between the two cultures (Rosenfeld, Personal Communication, 17.07.2017, p. XLIX, line 14-19).

In reply to the same question GB said:

...Japan was nearly destroyed by exactly that conflict, that the old, traditional family bonds dissolved entirely as a result of the developments in the society and economy. The young people that, let's say, stepped out of line. The old ones that were suddenly alone and this break in the socially established way has led the country into major problems. And if you take China as an example. What is still, let's say, totally untouched in China, is the respect the younger ones have for older age. ...And that is simply a matter of course. Let me say it like this; the Asian cultures are still much more; they are much more linked and grown together in generational networks than we are. With us, this really quite degenerated... (GB, Personal Communication, 15.12.2017, p. CIII, line 6-12 \& line 14-17).

Burkard Rosenfeld also commented on the lack of intergenerational bonds in Germany: 
...well, in the middle of Europe, in Germany families have been driven apart as a result of wars, abscondences and so on, as a result of the human character, ambition, employment and so on and that has an influence on the values of a society. In Mediterranean countries, this doesn't exist. Firstly, they didn't have war-like conflicts as in the middle of Europe. Secondly, it's the economic situation, the totally different economic situation in these countries that has led to the situation that Mr Asunto still travels to his mother in Calabria. Because frequently the family, several generations live in the same house... (Rosenfeld, Personal Communication, 17.07.2017, p. L, line 3-11).

Interestingly, Prof. Dr Schotten had not noticed any differences in the behaviour and attitude towards older people in the countries in and outside of Europe he had travelled to (Schotten, Personal Communication, 18.07.2017, p. 41). However, he attributed this less to the fact that there were none but more to him not having thought about this:

So far, I haven't noticed any differences. I have honestly never thought about whether, how the attitude towards ageing is since I don't feel that old. I don't feel that old. When I realise how old I am, then I know how old I am, but in this way, I don't feel that old, and that's why I don't know if the attitude there is different. I have never really noticed (Schotten, Personal Communication, 18.07.2017, p. LXXVI, line 13-18).

I found this remark of Prof. Dr Schotten illuminating in two ways. Firstly, it supports my finding (see chapter 7.2) that all interviewees do not perceive themselves as old and therefore older age and their status as pensioners have hardly affected their life course narratives and identity construction. Secondly, it explains the rather peculiar phenomenon that all respondents deeply perceptively and very critically analyse the socio-cultural and politicoeconomic foundations of western views on ageing but do so somewhat abstractly. In other words, all participants seem to be acutely aware of hegemonic narratives of ageing and particularly the hegemonic narrative linking ageing to decline but do not seem to, at any point in the interview, apply and link these realisations to their own status as retirees and older men.

To my mind, this also becomes clear when having a closer look at another crucial aspect in the construction of my male participants' identity which appears to be social comparison downwards with other men their age or older and upwards with younger men (Baur \& Luedtke, 2008; Festinger, 1954). As mentioned before, a primary arena for the establishment of male dominance is competitive sports. This does not seem to change with older age. Considering the high significance three of the participants place on doing sports, it appears that, apart from pursuing a meaningful occupation, competitive sports is a particularly effective means to confirm to oneself and one's social surrounding that one's physical health has not declined, that one is far from belonging to the scrap heap, and that one is much younger than the chronological age would suggest (Phoenix \& Griffin, 2015; Phoenix \& Sparkes, 2006; Sloan, Gough, \& Conner, 2010; Thompson, 2006; Wörsching, 1999). While 
in younger and middle age it is common to compete with men roughly your age, it is striking that my father, Burkard Rosenfeld and Prof. Dr Schotten chose to do sports in which they more or less explicitly compete with much younger men.

To illustrate this aspect, what follows is a comment of my father which I already quoted in chapter 7.2. but since it is so telling I decided to repeat it here:

...But with reference to feeling it is for me always a little bit the reflection of how others see me. And when I consider, when I consider that to ...two young men, who are in their mid-forties and they ... that they are really interested in continuing to play tennis with me, once a week. And that these meetings once a week clearly have a certain significance to them, then I have to say, then I am possibly, also in their eyes and in mine, not quite as old as the chronological age seems to indicate (Bühring, Personal Communication, 31.07.2018, p. CXIX, line 1-9).

He, however, also said: “...I don't have to go skiing until I am 85 or something necessarily. I don't need all that. At some point I will stop that," (Bühring, Personal Communication, 31.07.2018, p. CXXVI, 5-7)

Prof. Dr Schotten repeatedly mentioned how important sports and particularly running marathons had been throughout his life during the interview which he also, as the only interviewee explicitly linked to being male respectively to having "the male gene" (Schotten, Personal Communication, 18.07.2017, p. LXI, line 24). When I asked him if he had any aims for the future, he said: "Yes, I have the wish to run a marathon under four hours... That is a schizophrenic wish, I know that, but it is one," (Schotten, Personal Communication, 18.07.2017, p. LXX, line 5-6). I then asked him why it was a schizophrenic wish, and he replied:

Well, one could, of course, ask, if this must be...It's of course like this, I had previously always dreamt of running a marathon under three hours. I didn't manage. I managed three hours and six minutes... And then I didn't run so much anymore. And of course, when you...it is said that with increasing age you become one minute slower per ten kilometres every year. At a certain age and that is, of course, something and that is somewhat true if I think that I ran about three hours thirty years ago and now I can hardly reach four hours. That really is ... an ambitious aim for my age ${ }^{18}$ (Schotten, Personal Communication, 18.07.2017, p. LXXII, line 1-8).

Burkard Rosenfeld had also always done sports, first tennis and then he changed, due to injury, to golf, a sport he is highly passionate about and which he spoke about extensively and with great enthusiasm. What he especially likes about golf is that everybody can play with everybody and that it trains your character:

Yes, yes, naturally, it is a competition. And the clou is, and that doesn't exist in any other sports...well, in tennis when I have an opponent such as Dr Kill, for example, I have no chance... That feeling I don't need. There people who are very good and they

\footnotetext{
${ }^{18}$ As Prof. Dr Schotten told me recently, he actually managed to run a marathon at three hours and fifty-eight seconds last year.
} 
play against people who are, well, not so good and so that they compete there is the handicap...That is wonderful ...it is a wonderful sport and a great education for children, great education: discipline, consideration for others, fairness also against oneself(Rosenfeld, Personal Communication, 17.07.2017, p. XL, line 17-21 \& p. XLI, line 7-8).

GB does not follow any competitive sports, but he also mentioned that he walked the dog for two hours every day to underline his physical fitness. That sports do not play a significant role in the life-course narrative constituting his identity in older age is, in my view, due to the fact that according to his own testimony sports has never been an essential part of his life. It, therefore, follows that it also does not provide GB with a crucial basis for the construction of his older male identity. To me, it seems that GB mostly found meaning and social recognition in and through his work which then also could explain why it is particularly difficult for him to deal with the lack of a, in his eyes, meaningful occupation in older age (GB, Personal Communication, 15.12.2017).

Within the context of pitting one's physical strengths against younger men as a means to determine one's position within the male hierarchy in older age, I was particularly interested in gaining more insight into my interviewees' perception of the younger generation and their views on the possibility of intergenerational relationships - something which is also intensely in the focus of popular and academic discussions of older age (Gill et al., 2016; Gullette Morganroth, 2004, 2011; Jennings \& Krainitzki, 2015, unpublished; Johnson, 11.02.2013; Nelson, 2005). The topic of generational differences but also of connecting factors between the generations had occasionally been touched upon during the interviews mainly when, for example, Prof. Dr Schotten and GB talked about their time as lecturers or when the participants told me about their families or the present state of society (see section 7.2 and 7.3).

It was in these parts of the interview that it became clear that particularly GB found great satisfaction in being a mentor to the younger generation and that his work as a lecturer had changed his view of the younger generation. He, for example, said when telling me about his time as a lecturer at the University of St. Gallen:

I then also immediately completely revised my prejudices against the young generation. So, the 'couldn't be buggered-generation' was wrong. I take it back since I got to know young people who came to university with an incredible degree of commitment and enthusiasm and motivation (GB, Personal Communication, 15.12.2017, p. LXXXIX, line 10-13).

Shortly after he then said:

I always said, told the professor: 'You don't have to thank me. Really I would have to thank you. I learned a lot from the young people.' Yes, well, I think, if I provided them with something, it is nice. When they at some point later as adults and top managers 
say, well during my studies, I was taught by an old guru of purchase then that is great (GB, Personal Communication, 15.12.2017, p. XC, line 18-23).

When I asked him if he had the feeling that young people treated and viewed him differently in comparison to people their age or younger, he answered:

Yes. But I had the feeling that they were all extremely surprised how I responded to them, how I treated them. I always talked on eye level with them.... And I also really had the feeling that they understood that I didn't go there with the intention: I experienced all that 40 or 50 times myself, you can forget that. But with the honest intention, let's say, that with my insight into what the young person wants to achieve, I can guide their attention towards any land mines that might be placed somewhere. But that I didn't want to dishearten him but the contrary. I wanted to motivate him to discover his own strength and to stick by it and to demonstrate that (GB, Personal Communication, 15.12.2017, p. XCIII, line 4-14).

My father responded to the same question: "I think so, a little bit. I think so," (Bühring, Personal Communication, 31.07.2018, p. CXXVI, line 11). I then asked if he found that appropriate, and he replied:

Yes, the way, I have to come back to these two tennis mates of mine, how they behave towards me is on the one hand totally on the same level but in, with some other things characterised by a certain very subliminal respect. That is a very pleasant thing (Bühring, Personal Communication, 31.07.2018, p. CXXVI, line 13-16).

Prof. Dr Schotten denied that he had noticed that younger people treated him differently and then remarked when I asked him if he would like to receive this kind of respect:

Also, no, no, no. Well, I don't really have the feeling that I am not treated with respect somewhere....But...well, sometimes I obviously think when you see 10-12-year-old teenagers on the tram or on the train, and you see how they sprawl and nobody thinks of getting up for an older person. That does annoy me. I honestly have to say. But that doesn't refer to me but in general. That you say, that really is bad (Schotten, Personal Communication, 18.07.2017, p. LXXII, line 2-12).

I then probed further by asking if he would find it appropriate that younger people paid him and his older age high respect, and he answered: "Not really, no...I don't know. I don't know. If I had the feeling that was respected simply because of my age that would be too little for me," (Schotten, Personal Communication, 18.07.2017, p. LXXIV, line 1-2).

Burkard Rosenfeld's response to the same question I already quoted above within the context of determining one's own age by social comparison (ompare chapter 7.2). He had said that he felt that younger people, indeed, treated him differently to people their age or younger people and explained that with the fact that younger people are in a totally different life stage and that older people even if they did not do that on purpose had a habit of being selfrighteous and dominant which made a balanced relationship problematic (Rosenfeld, Personal Communication, 17.07.2017, p. 30-31). He then continued by saying: "You want 
to be on the same level, but you are not," (Rosenfeld, Personal Communication, 17.07.2017, p. LII, line 2-3)

His view resembles Hannah Hamad's (Gill et al., 2016) concept of toxic intergenerationally without, however, being quite as toxic as she describes it. Burkard Rosenfeld considers the hierarchical power relations between old and young as characterised by the insistence of older people on their status of dominance which they established at early stages in one's life on the basis of the higher levels of experience, knowledge and power - something which he perceived as nearly impossible to change which he attributed to the inability of older people to give up the superior socio-cultural position they occupied over long periods of their lives even if they might not be aware of this themselves. I found his thoughts on intergenerational relationships particularly illuminating since, in my view, they accurately capture the main hurdle for any fruitful cooperation between younger and older people, although I believe that younger people have as many problems with getting to grips with renegotiating the distribution of power when they become more and, for instance, their parents become less powerful (Lindley, Harper, \& Sellen, 2008).

My father's reply to my question if he believed to be at an advantage in comparison to younger people supported Burkard Rosenfeld's view on the continued status of dominance older people purport:

That would be bad if that wasn't the case...First of all in areas which have to do with relationships between people. The evaluation of how to handle these, these human relationships. With this I certainly have a bit more and that all is called experience. In general how to deal with, let's say, problems, how to go about problems, how to perhaps start a problematic task and on the other hand in another perhaps not (Bühring, Personal Communication, 31.07.2018, p. CXXVII, line 5-11).

I then also asked him if he believed to be at a disadvantage in any area when compared to younger people, he replied:

Well, the way I see current society to a limited degree. That I really believe...Well, I think, if you could say they still have their life ahead of them and such things but what life offers, I don't know if that is such a big advantage. Well, in some way, I can't really, I really can't take a stand on this since ...I cannot relate to that. That is not a criteria for me. Well, I don't want to bring up a family or have a career now. I don't want all that (Bühring, Personal Communication, 31.07.2018, p. CXXVII, line 15-22).

GB had already reflected on the relationship between generations when I had asked about where he thought western understanding of older age came from. In the continuation of the above-quoted citation, he said:

$[\ldots .$.$] The other thing is that I think that the young people, the younger generation will$ for the same reasons have a problem, with the growing strain of social insurance contribution, to be soaked for that. I believe that the conflict between generations will 
increase as a result of the economic situation... Then the old will increasingly not understand the young .... and the young will more and more lose respect for older age. Also so they can somehow make ends meet (GB, Personal Communication, 15.12.2017, p. CI, line 19-23 \& CII, line 11-13).

When I then enquired if he had experienced this kind of generation gap in his closer social environment, he said:

No, absolutely not. What I sometimes jokingly say to my daughter and to my son is: Listen, you are now in your prime and also experienced managers, and you earn good money, but I am not senile. I can still think straight. Because sometimes there is somehow the attitude that you have the impression that your children now believe that they need to make decisions for you because you are not able to do the right thing yourself. But besides that, let's say, no (GB, Personal Communication, 15.12.2017, p. CII, line 16-20 \& p. CIII, line 1-2).

All in all and despite their rather different responses, it seemed to me that all participants did not generally disapprove of the younger generations but felt a certain distance to you younger people and their way of life. This became, for example, quite clear in the last quote I cited of my father but also in the remark of Burkard Rosenfeld who said: "But I admire the young people how they accept this und I have to laugh out loud when I drive somewhere, and I see children and adults running around," 19 (Rosenfeld, Personal Communication, 17.07.2018, p. LII, line 10-12) Also GB said something similar:

...It is not my world anymore. I also can't imagine that that would make me happy. But the kids grow up with this. You see that today when you go into a restaurant. Look at the tables. Nearly everybody even when there are three, four people on the table. Everybody has his mobile phone and looks at his mobile phone and send some SMSs or something like that. But that they talk to each other is more the exception than the rule (GB, Personal Communication, 15.12.2017, p. CIV, line 10-15).

Although the interviewees do generally view younger generations with a certain amount of sympathy, in my view, the above quotations indicate that all interviewees feel some scepticism towards the way they live their lives. It sometimes seems as if the participants view younger people like a totally different species who live in a world which the respondents can, in many ways, not relate to anymore. As such, their comments with reference to generational cooperation somewhat mirror the hegemonic narratives. Public discourse insists on communicating that older people drain the social security systems and stay glued to jobs which belong to younger people whilst the older population is portrayed as bemoaning a certain lack of respect for their age and linked experiences (Pickard, 2019). The interviewees' responses are milder and more balanced than what is representative for the dissemination of narratives linked to intergenerational cooperation, but they are, to my mind, nevertheless based on certain hegemonic understandings of the generation gap

\footnotetext{
${ }^{19}$ with their mobile phones - the reference was clear since we had discussed this issue right before
} 
separating the older from the younger (Ayalon \& Tesch-Römer, 2018; Jönson, 2012; Nelson, 2005; Palmore, 2001).

Within this context particularly my father's frustration with the general societal view which links age to decline and thereby devaluates older people whilst everything related to youthfulness is set as the all-encompassing standard one is judged against also became apparent when he talked about the representation of older people in advertisements:

I find this image which is transmitted in advertising that there are either ill people who are helped or people that are also helped by something so that they are happier. That means they are always in a condition that is somewhat pitiable or not even pitiable but definitely give a reason for improving it so that there is somebody who is old and happy without ailments that doesn't exist in advertising (Bühring, Personal Communication, 31.07.2018, p. CXXX, line 14-19).

Except for above quote and Burkard Rosenfeld's above-cited reference to a film he enjoyed watching and in which he admired the wise actions of the older protagonist (Rosenfeld, Personal Communication, 7.07.2017, p. XLVII, line 15-16), the interviewees had, even when probed, strikingly little to say about the media portrayal of older age. I attribute this to their particular media usage. As far as I could deduce from the interviews, all participants mostly watched sports programmes on TV and when going to the cinema, at least, Prof. Dr Schotten, Burkard Rosenfeld and my father, preferred independent films to mainstream blockbusters and all three clearly voiced that they favoured reading over watching TV. It is therefore not overly surprising that these three interviewees had neither watched The Expendables trilogy nor that Hollywood films in general and mass-produced Hollywood action films, in particular, were not their favourite genre (Rosenfeld, Personal Communication, 17.07.2017, Schotten, Personal Communication, 18.07.2017 \& Bühring, Personal Communication, 31.07.2018).

What, however, is surprising is that all men favoured a view of older masculinity which in many ways resembles the contextualisation of the older protagonists in The Expendables although they did not view muscularity and the establishment of male dominance by violence and aggression as representing their understanding of masculinity. When I asked Burkard Rosenfeld what he thought about older actors being the protagonists in action films he said: “That doesn't impress me," (Rosenfeld, Personal Communication, p. XLVII, line 6). When I enquired if muscles were part of Prof. Dr Schotten's understanding of masculinity he admitted that this was something which was generally "...exemplified and shown," (Schotten, Personal Communication, 18.07.2017, p. LXXVIII, line 3) as being linked to masculinity but then said: "For me this is repellent. So no factor for envy," (Schotten, Personal Communication, 18.07.2017, p. LXXVIII, line 5). 
However, integrity, meaningful occupation which contributes to society and personal autonomy and control clearly form essential aspects of the interviewees' identity construction of masculinity. As discussed above, these attributes which were significant in the construction of their younger and professional construction of masculinity appear to still form a crucial basis of their identity construction in older age. At the same time, wisdom and being a mentor to the younger generation represents an extension to their current views of masculinity and the only concession to their older age. In addition, their views on intergenerational relationships appear to reflect what the contextualisation of the cooperation of young and old in The Expendables trilogy is informed by, namely that it is possible but only when based on a clear hierarchical structure in which the older are accepted as the unquestionable authority. I, therefore, want to end this section with what GB said about The Expendables trilogy since I find it illuminating not only for his view of older masculinity but also for the other participants' approach to their older age despite the fact that they have not watched the films. The following is GB's reply to my question if he could relate to the portrayal of the protagonists in The Expendables:

Sure. Yes because, I mean, there is somehow still ... That has meaning. That is somehow with substance where you can say boah. Well, despite older age. They are still getting somewhere. They have no problems with their self-identification. Or they have no problem with motivating themselves. They know that they are old and they know that, let's say, their abilities have a natural limit. But within these limits of their abilities, they fully take part (GB, Personal Communication, 15.12.2017, p. CV, line 14-18). 


\section{Conclusion}

\subsection{Older Men Staring at Age in a Neoliberal Society}

When I embarked on this research project, I was motivated by the strong desire to gain a better understanding of how the life-course narratives of the four German men I interviewed developed within and around western hegemonic socio-cultural narratives linked to masculinity and ageing in general and with particular reference to the respective sociocultural frames I identified in The Expendables trilogy. After having established the relevance of my research topic and clarifying my research position and my research design I thus first discussed key concepts and approaches linked to ageing and masculinity to then explore the narratives underlying the portrayal of the older cast in The Expendables trilogy. Chapter 3 of this thesis focused on the significance of master narratives on individual lifecourse narratives and current master narratives linked to older age in western countries. I found that today master narratives of western societies are informed by the core values of neoliberalism: productivity, consumption and individual autonomy (Chomsky, 1999; Harvey, 2007a). Within this context, I highlighted the significance of social comparison upand downwards for establishing one's position in society and for motivating the optimisation of oneself by consumption, productivity and, most importantly, by incessantly reassuring oneself and one's social environment that one is the agent of one's own life (Corcoran et al., 2011; Festinger, 1954; McDonald et al., 2017).

In this chapter, I also established that understandings of older age in western countries unfold within two contrasting frames. On the one hand, older age in western societies is viewed as a life stage mainly characterised by the decline of one's physical and mental abilities and a subsequent a lack of control over body and mind, dependency on others, limited financial means and a subsequent loss of status and privileges (Chivers, 2011; Cruikshank, 2013; Phoenix \& Griffin, 2015). This negative perception of ageing is purported by the fact that in western societies dying and death - one of the last taboo topics in the western hemisphere mark an utter loss of control and a level of ambiguity and insecurity which is unprecedented in our lives (Calasanti \& King, 2015; Joyce et al., 2015; Tulle, 2015). On the other hand, the concept of successful ageing is based on the view that it is possible to manage the ageing process effectively. This is possible when one has followed a healthy life-style throughout one's life, has wisely planned for older age and ensured sufficient financial means for retirement, continues to be a productive member of society, and consumes products and services aiding physical and mental fitness in older age (Featherstone, 1995; Gilleard \& Higgs, 2000; Gullette Morganroth, 2004; Katz \& Calasanti, 2014; van Dyk, 2014). 
In chapter 4, I examined constructions of masculinity across time, in different cultural settings and at different individual life stages with a particular focus on the German sociocultural context and constructions of older male age. I concluded that although constructions of masculinity vary across time, within individual life-courses and in different cultural settings (Connell, 1993; Connell, 2005), there are key aspects which link the magnitude of different understandings of hegemonic masculinity.

Firstly, narratives of hegemonic masculinities are primarily constructed in opposition to socio-cultural understandings of femininity. Men are thus perceived the more manly, the less feminine their behaviour and looks are (Connell, 2005; Schippers, 2007). Secondly, dominant constructions of masculinity crucially depend on incessantly reaffirming one's hegemonic status by displaying control of one's own body and mind as well as by subordinating others, especially women but also other less manly men (Cheng, 1999; Connell, 2005; Kimmel, 2001; Pyke, 1996). Lastly, competition and the level of performance, particularly in sports and within a professional environment, plays a significant role in the establishment of masculine status (Baur \& Luedtke, 2008; Bourdieu, 2001).

I also identified that German men born between 1939 and 1946 were as teenagers and young adults exposed to the Prussian ideal of a soldier as well as the 'Bildungsbürger' conception of masculinity. Both of these hegemonic constructions of masculinity are based on a clear separation between the private and the public sphere and on viewing the man as the provider and the woman as the carer for the family. The Prussian soldierly ideal of masculinity emphasised characteristics such as discipline, mental and physical strengths, integrity and emotional and cognitive control whilst being based on a strict and unquestionable male hierarchy (Colvin \& Davies, 2008; Willems, 1986). The 'Bildungsbürger' understanding of masculinity continued to emphasise attributes such as discipline, emotional and cognitive control and rationality but physical dominance and aristocratic inheritance became increasingly linked to marginalised understandings of masculinity (Gardiner, 2004).

During the early stages of their career, the interviewees then witnessed the increasing popularity of a masculinity construction demanding of men to show their emotions and, in general, to give in to their feminine side (Gotto, 2017). In the last two decades of their working life the interviewees were exposed to a hegemonic construction of masculinity - the transnational masculinity construction - substantially dependant on tirelessly proving and displaying dominance without, however, utilising physical violence to enforce subordination (Connell \& Messerschmidt, 2005; Fitterer, 2013; Fletcher, 1997; Hall, 2002). In the final section of chapter 4 , I concluded that ageing and retirement robs older men, especially if they used to perceive themselves as belonging to the hegemonic class of men, of a meaningful role supporting the feeling of all-encompassing agency, linked status and 
importance. Consequently, older age not only seriously impedes men's claims for patriarchal privileges and hegemonic status, but it also challenges their sense of self (Beynon, 2001; Chivers, 2011; Kimmel, 2001).

In chapter 5, I focussed on the analysis of The Expendables trilogy to gain a better understanding of the socio-cultural narratives linked to ageing and masculinity, which are at work in these three films. I found that masculinity in The Expendables is constructed by emphasising the meaningfulness of the protagonists' job, the extraordinarily fit mental and physical shape of the older protagonists, their tough-guy manliness and the high standards of morality the Expendables team live by.

The clear sense of justice and their willingness to fight for the interests of the marginalised, discriminated, and vulnerable of the Expendables team stresses their superiority over other men, particularly men who represent the current masculinity ideal of transnational masculinity. At the same time, the nobility of their missions allows the members of the team to activate nearly superhuman abilities and to stay in emotional and cognitive control even in the most challenging times. Subsequently, this enables them to subordinate and dominate anybody whose actions are not motivated by such noble aims and who are thus either characterised by a total lack of morality and linked loss of control or who are not brave enough to break out of the corset of the politically correct blueprints of current understandings of hegemonic masculinity (Donnar, 2016; Feasey, 2011; King, 2007).

In addition, I argued that on the one hand the older protagonists in The Expendables are portrayed as wise and emotionally more balanced than the younger cast whilst on the other hand, the older cast is shown as cynical, resigned and embittered by the experiences they made during their life-course - all attributes linked to the master narrative of perceiving older age as decline (Gullette Morganroth, 2004; Woodward, 2003). Nonetheless, the contextualisation of older age in the trilogy is by and large based on the narrative of successful ageing. The films essentially communicate that to age successfully as a man and to subsequently ensure hegemonic status also in older age men first and foremost need to prove and maintain their unremitted personal autonomy and agency since this is the crucial pre-condition for confirming one's hegemonic position (Beynon, 2001; Bourdieu, 2001; Connell \& Messerschmidt, 2005; Fausto-Sterling, 1997).

Lastly, I argued that The Expendables trilogy is exemplary for what Slavoj Žižek (2008) has termed the ptolemization of neoliberal values. On the surface, the films seem to critically question the values and standards of current society which the master narratives of ageing and masculinity are based on. The portrayal of the older cast in The Expendables team appears to present a narrative contrasting current hegemonic narratives of ageing and particularly of masculinity which serves the audiences' demand for a critical attitude towards 
neoliberal values (Žižek, 1997, 2008). A more in-depth look, however, reveals that although the masculinity scripts underlying the depiction of the protagonists are old-fashioned and archaic, they are substantially informed by key neoliberal values. As such the films criticise the nowadays hegemonic understanding of masculinity characterised by diplomacy and pseudo-pacification (Hall, 2000, 2002) and, at the same time, perpetuate neoliberal standards, such as individual autonomy and productivity respectively performance orientation which are crucial in allowing men access to patriarchal privileges regardless of their chronological age (Feasey, 2011).

During the interviewing stage, the openness, depth and extent of the interviewees' accounts allowed me to gain novel and relevant insights into the lifeworlds of the four older German participants who took part in my study. This subsequently enabled me to understand better how the socio-cultural narratives of ageing and masculinity in western cultures I had previously identified, have affected the identity construction of my interviewees.

Since concepts of masculinity and particularly hegemonic understandings of masculinity are highly relational, unstable and strongly influenced by the respective historical setting, individual definitions of masculinity primarily depend, as highlighted in chapter 4 of this thesis, on delimiting oneself from constructions of femininity as well as from other men who do not fit the current standards of hegemonic masculinity (Connell, 2005). Only through comparing and contrasting oneself with other men and women and through the subsequent domination and subordination of others can men establish and retain a dominant status (Bourdieu, 2001; Schippers, 2007).

One of the significant findings of my research has been that this perception is carried forward into older age and continues to form the foundation identity in older age. My interviewees based their definitions of masculinity on the clear separation of male and female attributes and spheres of activity - something all of them seemed to perceive as a naturally determined differences (Rosenfeld, Personal Communication, 17.07.2017, Schotten, Personal Communication, 18.07.2017, GB, Personal Communication, 15.12.2017 \& Bühring, Personal Communication, 31.07.2018). They all expressed that in their view women were naturally more suited to being mothers and to taking care of the household, the family and their emotional needs whilst the natural predisposition of men enabled them to be active in the public arena, to secure the financial stability of the family by following a meaningful and lucrative occupation permitting a high level of social recognition and status (Rosenfeld, Personal Communication, 17.07.2017, Schotten, Personal Communication, 18.07.2017, GB, Personal Communication, 15.12.2017 \& Bühring, Personal Communication, 31.07.2018). This research also confirms that the socio-cultural view which assigns attributes such as activity, rationality, determination and the will to succeed to men and passivity, emotionality 
and a natural need and talent for being motherly and caring to women has in the past decades not changed significantly (Bourdieu, 2001; Chambers, 2005; Negra et al., 2007; Tasker \& Negra, 2007). In fact, the view of naturally determined gender relations is, probably due to its reference to biological predisposition, particularly persistent and till today at the core of many hegemonic narratives linked to masculinity and femininity (Fausto-Sterling, 2000; Lorber, 1993). As a result, women today are faced with the challenge to, on the one hand, pursue a career which is transmitted in popular culture as a prerequisite for personal fulfilment and, on the other hand, expected to become mothers and take care of the family and the household since apparently that is in their genes and therefore only through motherhood and taking care of a family can women find true fulfilment (Lorber, 1993).

This view of gender relations was also evident in my interviewees' accounts. All interviewees welcomed that their wives had worked. In fact, two of them stated that they perceived this as essentially supporting a healthy relationship (Rosenfeld, Personal Communication, 17.07.2017 \& Schotten, Personal Communication, 18.07.2017). However, it also became apparent that the interviewees saw the primary responsibility of their wives in catering for the emotional needs of the family whilst they insisted on rationality, activity and performance orientation as being predominantly male traits (Rosenfeld, Personal Communication, 17.07.2017, Schotten, Personal Communication, 18.07.2017, GB, Personal Communication, 15.12.2017 \& Bühring, Personal Communication, 31.07.2018). The high significance my interviewees attributed to control, expressed by stressing the attributes of activity, rationality and personal autonomy is, in my view, both the outcome of their socialisation and the specific conceptions of masculinity they were affected by throughout their life-course and of current understandings of masculinity (Connell, 1993; Connell, 1998; Connell, 2005). This supports my assumption that the interviewees' life-course narratives were majorly affected by The Prussian ideal of a soldier and the 'Bildungsbürger' conception of masculinity which are both based on a clear separation between the public respectively male and the private respectively female sphere.

Nevertheless and despite the fact that the interviewees' view of masculinity reflects many aspects linked to the 'Bildungsbürger' conception of masculinity, interestingly, all interviewees rejected being diplomatic and explicitly uttered their dislike for situations in which they were forced to do so when employed. They clearly felt that this was an attribute which had little to do with their perception of masculinity. Within this context, they expressed their disapproval for the type of diplomacy characterised by being dishonest and betraying oneself and one's values in the interest of gaining more financial and/or social power. What seemed to be much more in line with the interviewees' view on masculinity and male practices within a business context were honesty, staying true to oneself, integrity 
and teamwork (Rosenfeld, Personal Communication, 17.07.2017, Schotten, Personal Communication, 18.07.2017, GB, Personal Communication, 15.12.2017 \& Bühring, Personal Communication, 31.07.2018). These attributes are in stark contrast to the fiercely competitive attitude, immorality and ruthlessness nowadays needed to enable a career in multinational corporations and in neoliberal society in general (Chomsky, 1999). ). Thus, a significant finding of my investigation is that my interviewees' identity construction in older age is, at least partly, based on a renunciation of the current conception of transnational masculinity and linked amoral conviction that success justifies almost any means (Chomsky, 1999; Gergen \& Gergen, 2000).

At the same time, it must be highlighted that stressing authenticity, working together and personal honourability as essential criteria in the participants' conception of masculinity also allowed them to maintain in control of themselves and others - something in jeopardy when being diplomatic since it entails giving up some of one's independence and control to others to enable reaching a specific goal. This might also be necessary when working in a team. During the interviews, however, the interviewees argued that it was essential for tasks to be clearly separated and assigned and that each member of a team needs to trust the others and their expertise. In this model of teamwork, individuals would therefore not question their decisions or right to rule in their respective professional field (Rosenfeld, Personal Communication, 17.07.2017, Schotten, Personal Communication, 18.07.2017, GB, Personal Communication, 15.12.2017 \& Bühring, Personal Communication, 31.07.2018).

This conception of masculinity mirrors the socio-cultural narratives at work in the portrayal of the older protagonists in The Expendables trilogy. Their depiction seems to represent an increasingly marginalised understanding of masculinity in which men are brave and heroic and fight for justice, often in disregard of rules and values set by an anonymous state authority, and defend the defenceless - predominately women and children. (Heron, 2008; Jeffords, 1994; Poiger, 1998). However, as highlighted in my analysis of The Expendables films, when examining contextualisations of masculinity in public discourse and particularly in mass media outlets it is astonishing how often the portrayal of men is based on precisely these characteristics.

This heroic and somewhat unrealistic image of masculinity presented in the media is the outcome of catering to audiences who are increasingly aware of neoliberal indoctrination, whilst the lack of alternative value systems leaves them disoriented, desperately seeking stability and unambiguous behavioural guidelines (Žižek, 2008). The revival and public transmission of more conventional understandings of femininity and masculinity which, as they are less ambivalent, offer a more effective, even if idealised, templates for one's own behaviour. These constructions cannot merely be categorised as the expression of a 
marginalised and redneck conception of gender relations (Boyle \& Brayton, 2012). Instead, it seems to me that both narratives, the transnational masculinity and the more conventional understanding of masculinity, exist side by side and are equally visible in public discourse. As such, both must be viewed as socio-cultural frames currently available for (older) men to draw on to construct their identity (Greven, 2010; Griffin, 2005; Holt \& Thompson, 2004; Leek, 2016; Mac an Ghaill, 2003).

As I discussed in chapters 3 and 5, public discourse insists on both the possibility of managing the ageing process and on the individual duty to do so, regardless of specific personal circumstances such as physical or mental impairments or a lack of financial resources, which majorly impact one's 'success' at ageing. Not following the dictate of successful ageing and giving in to decline leads to social exclusion and condemnation in this model, and how to embody and realise the demands set by the paradigm of successful ageing is the sole responsibility of each older individual (Gilleard \& Higgs, 2000; Gullette Morganroth, 2004, 2011; Segal, 2014; Spector-Mersel, 2006).

The markers of successful female ageing perpetuated in popular discourse centre around maintaining attractiveness, sexual desirability and on using their alleged talent for caring for others to the benefit of society (Chivers, 2011; Richardson, 2016, 2019; Richardson \& Wearing, 2014). In contrast to this, physical and mental control are at the core of the sociocultural narratives of older male age since these aspects are vital pre-conditions for confirming one's hegemonic position in the domination and subordination of others. This is particularly true with regards to men who used to represent many characteristics linked to hegemonic constructions of masculinity such as my interviewees (Sandberg, 2011; Saxton \& Cole, 2013; Schippers, 2007; Thompson, 2006).

My research has confirmed this finding. In the interviews, it became clear that all interviewees predominantly filled the 'roleless role' (Cruikshank, 2013, p. 42) of being an older man, by relying on tested and tried conceptualisation of themselves as men which characterised their understanding of masculinity throughout their lives (Rosenfeld, Personal Communication, 17.07.2017, Schotten, Personal Communication, 18.07.2017, GB, Personal Communication, 15.12.2017 \& Bühring, Personal Communication, 31.07.2018). Although the life-course narratives of my interviewees differ in many ways, managing the ageing process and controlling mental and physical decline by activity and by living a meaningful life were recurrent themes in all interviews. The participants viewed these attributes as not only predominantly linked to masculinity but they also seemed to represent a crucial basis for their life-course narratives in young and middle-age as well as in older age (Rosenfeld, Personal Communication, 17.07.2017, Schotten, Personal Communication, 18.07.2017, GB, Personal Communication, 15.12.2017 \& Bühring, Personal Communication, 31.07.2018). 
My research thus evidenced that affirming to oneself and the surrounding environment full control of oneself continued to be, as in earlier stages of my participants' lives, a significant aspect in the construction of a positive older identity of the interviewees. Interestingly, within this context, who is controlled and who is dominated seemed to be highly relevant for the interviewees.

In contrast to women, men in the age group of my participants spent most of their time on the job and resultantly their life-course narratives and linked individual identity constructions appear to be based predominantly on the social recognition and feedback received in their work environment (Baur \& Luedtke, 2008). Professional activity and achievement were perceived by all interviewees as particularly relevant in confirming to themselves that older age had not majorly impacted their capabilities, whilst also being the most effective means to ensure continued mental fitness and to keep any signs of ageing in check. To a lesser degree reading fictional and non-fictional literature of a high complexity level, and continuing to expand one's horizon by travelling to destinations outside Europe, appeared to serve a similar purpose, at least for Burkard Rosenfeld, Prof. Dr Schotten and my father. Despite the dislike, all interviewees expressed for the display of exaggerated muscularity, doing sports regularly and challenging oneself physically continued to be of utmost importance in the lives of Burkard Rosenfeld, Prof. Dr Schotten and my father (Rosenfeld, Personal Communication, 17.07.2017, Schotten, Personal Communication, 18.07.2017 \& Bühring, Personal Communication, 31.07.2018).

In many ways, they seemed to view sports and particularly competitive sports in combination with a healthy lifestyle as a crucial prerequisite for managing and controlling age-related physical decline. Only GB was neither professionally active anymore nor did he practice any sports intensely or had other time-consuming hobbies, and he seemed to suffer immensely by the inability to fill the gap left by being professionally active with another meaningful activity which could support him feeling significant and valuable (GB, Personal Communication, 15.12.2017). Another main finding of my investigation is thus that proving unremitted power to other men of a similar or younger age and the positive social recognition received outside of their private context and by other men represents a major constituent in the life-course narratives the interviewees constructed in their older age (Beynon, 2001; Bourdieu, 2001; Gray, 2000; Hearn, 2014).

An additional outcome within this context was that, in contrast to what I had assumed, my participants' attitude towards death was neither determined by their religious inclination nor characterised by fear due to the high level of uncontrollability and insecurity related to death (. In fact, GB, the only interviewee who described as characterised by a sound faith in God, was also the only interviewee who admitted feeling some fear of death. He explained this by 
the fact that, although he believed in God, he did not really know what would happen after death (GB, Personal Communication, 15.12.2017).

It was precisely this inability to know what happens after death which the other participants mentioned as the reason for not being afraid of death. Prof. Dr. Schotten, Burkard Rosenfeld and my father more or less strongly expressed that it was futile to worry about something one has no access to and consequently cannot know about. This indicates that how my interviewees felt towards death was less dependent on their faith and more on the significance the attributed to rationality. It follows that particularly my father and Prof. Dr Schotten, in whose identity construction rationality played an especially crucial role, do not see any sense in thinking or being afraid of something which goes beyond the horizon of humanly accessible knowledge (Rosenfeld, Personal Communication, 17.07.2017, Schotten, Personal Communication, 18.07.2017 \& Bühring, Personal Communication, 31.07.2018). Nonetheless, all participants admitted to being horrified by the possibility of losing physical and/or mental abilities as a result of age-related illnesses (Rosenfeld, Personal Communication, 17.07.2017, Schotten, Personal Communication, 18.07.2017, GB, Personal Communication, 15.12.2017 \& Bühring, Personal Communication, 31.07.2018). This ties in well with one of my main findings since age-realted illnesses often lead to a decrease and, in the worst case, loss of personal autonomy. As repeadedly discussed throughout this thesis, control and personal autonomy represent a crucial basis for male identity constructions throughout their lives. That the image of being helpless and fully depenedent on others horrifies the interviewees does, therefore, not surprise and confirms that agency and personal autonomy, indeed, mark a particularly significant constituent in the identity construction of my male participants.

However, none of the participants expressed feeling any major limitations due to older age, yet. They, therefore, did not apply any characteristics tied to viewing ageing as decline to their own older identity. Instead, they emphasised characteristics such as wisdom and placidity which in neoliberal societies of the West, characterised by their emphasis on speed, progress and on keeping up with the newest often technological advances (Baars, 2015), are rather out of fashion and representative for a view of ageing which is today more prevalent in Asian societies (Blaikie, 1999; Eckersley, 2006; Fairclough-Isaacs, 2015; Weedon, 2004).This focus on characteristics which, although they seem to count little in their own culture (Grossmann et al., 2012; Woodward, 2003), are not only primarily based on emotional and cognitive control (Woodward, 2003) but also on the one thing young people cannot compete with, namely experience, allowed the participants to retain a positive and consistent self-concept of themselves as older men, despite the often negative view of the physical and mental changes linked to ageing in the west (Lindley et al., 2008). That the 
portrayal of the older cast in The Expendables drew on similar narratives also supports the assumption that linking older age to serenity and emotional balance is one of the few positively connoted socio-cultural accepted scripts available to older men with which to construct a positive older identity (Cruikshank, 2013).

In my study, I also identified that the construction of a positive older identity was facilitated by the clear distance all participants seem to feel towards the younger generation (Rosenfeld, Personal Communication, 17.07.2017, Schotten, Personal Communication, 18.07.2017, GB, Personal Communication, 15.12.2017 \& Bühring, Personal Communication, 31.07.2018). None of the participants expressed an attitude which resembles the toxicity of intergenerational relationships. Hannah Hamad (2016) describes, and in fact, all believed to get on quite well with younger people. Nonetheless, in each interview, there was a point in which the interviewees stated that they would not like to be young again or to have a family and work in current times, that it was not their world anymore or that younger and older people are simply on different levels (Rosenfeld, Personal Communication, 17.07.2017, Schotten, Personal Communication, 18.07.2017, GB, Personal Communication, 15.12.2017 \& Bühring, Personal Communication, 31.07.2018). It was my impression that distancing themselves from the younger generation and their way of life was a strategy which permitted the interviewees to invalidate their social evaluation on the basis of the still prevalent sociocultural perceptions linking older age to decline, inferiority and uselessness. As I illustrated in chapter 5 of this thesis, this strategy mirrors the contextualisation of the older male characters in The Expendables trilogy and their attitude to the younger generation.

Another key outcome of my investigation is the finding that despite the precision with which the interviewees dissected current socio-cultural norms and values particularly with regards to societal attitudes towards ageing, they seemed to apply these insights to their own lives seldomly. Whilst they disapproved of the dictate of productivity, consumption and autonomy in current society they placed high significance on agency, on exercising control over the ageing process through discipline, through challenging oneself mentally and physically in a professional or intellectual context or in sports, and through living a healthy lifestyle. In this, they essentially mirror the basic conceptions of successful ageing (Crosnoe \& Elder, 2002; Katz \& Calasanti, 2014). This indicates that the critical attitude towards society evident in the accounts of my interviewees as well as in the narrative construction of the older cast in The Expendables must, despite appearing to be the opposite, in fact, be viewed as a dominant socio-cultural narrative in current western societies (Žižek, 2008).

Another significant finding of my study was that comparing oneself with others, often with people in third age or fourth age, appears to be a crucial strategy for maintaining a positive self-perception as older men. As highlighted in chapter 3 of this thesis, this strategy is deeply 
infused with neoliberal ideology. That the interviewees applied the strategy of social comparison (Festinger, 1954) to inform their self-perception in older age is evidenced by the fact that they all expressed that they did not feel as old as their chronological age would suggest - an assessment mainly based on the feedback received in their social surroundings and on evaluating oneself and others on the basis of the principles significant in a meritocracy (Rosenfeld, Personal Communication, 17.07.2017, Schotten, Personal Communication, 18.07.2017, GB, Personal Communication, 15.12.2017 \& Bühring, Personal Communication, 31.07.2018).

In basing their life course narratives as older men on the criteria indicative of a successful performance of masculinity in previous stages of their lives, their identity construction also mirrors essential constituents of the successful ageing paradigm. They attributed high significance to remaining autonomous, productive and active in old age and understood this as prerequisite for the development of a positive older masculine identity. These aspects determine masculine status within male hierarchies but simultaneously they are also crucial benchmarks determining older men's success at ageing. My interviewees, thus, continued to rely on the templates for successful male performances characterising earlier stages of their lives and since they evaluate themselves "by competitive business standards" (Gravagne, 2013, p. 30) they fulfil many of the socio-cultural standards linked to successful male ageing. At the same time, however, viewing the participants' life-course narratives in older age as being based solely on socio-cultural frames setting the neoliberal parameters for successful ageing and hegemonic masculinity constructions would, in my view, not adequately capture their approaches towards the construction of an older male identity. Particularly their insistence on not feeling old and certainly not as old as their chronological age suggests that the successful ageing paradigm did not provide the interviewees with an entirely sufficient template for their lives in older age (Ayalon \& Tesch-Römer, 2018; Baars, 2013; Gullette Morganroth, 2008).

In addition to before-mentioned aspects, in chapter $3.1 \mathrm{I}$ also argued that the use of chronological time to assign people to cohorts and generations which set the standards for how one should feel, behave and what one should have achieved at what stage at any particular life stage contributes to the homogenisation and marginalisation of older people. In contrast, Helen Moglen's (2008a) views older age as informing only one of the many selves one develops, embodies and performs over the life-course at different times, in a variety of contexts and largely independently from chronological age which in combination then form one's identity.

All interviewees described the process of ageing as slow and gradual and as something one successively adapts to. None of the interviewees experienced their life as retirees as a life 
stage entirely detached from their previous lives but much rather as a logical continuation of what they had been and done before retirement. During this period, their priorities and attitudes had - as in other life stages - marginally changed due to changes in their personal circumstances and the increasing feeling that one's lifetime is limited.

When I asked my father if he believed to be successfully ageing, he said: "Yes, because I do appreciate yesterday and also think about it and also bring it up once and again but that I also have many experiences that define the now...I also live now and not only in the past," (Bühring, Personal Communication, 31.07.2018, p. CXXI, line 9-11 \& line 13). His definition of successful ageing emphasises the inextricable link between the past and the present - a relationship which can only inadequately be captured by chronological time. That my father's reinterpretation of successful ageing, as well as the life-course narratives of all participants, mirror Helen Moglen's (2008a) concept of transageing suggests that this approach might, indeed, be better suited to reflect the individual experiences of older people than chronological time, and the subsequent categorisations of older population groups on the basis of age cohorts (Gullette Morganroth, 2011; Jennings \& Krainitzki, 2015).

At the same time, my father's definition of successful ageing also highlights how the interviewees adapted and altered hegemonic narratives of ageing to develop their own positive older male identity. Although all participants clearly drew on neoliberal concepts of ageing and masculinity to construct their identity in older age, their reflections on their personal approach towards older age and towards handling its effects illustrates that the socio-cultural narratives perceived as hegemonic in any respective society are not a one-way road. Although there can be no doubt that socio-cultural frames have an enormous influence on the way one constructs one's identity, how these narratives are interpreted, adapted and changed to fit one's individual situation and worldview is up to each person (Foucault et al., 2008; Lemke, 2014).

Three of the participants, GB, Burkard Rosenfeld and Prof. Dr Schotten, in hindsight, expressed regret with regards to having spent such a high amount of time at work instead of dedicating more time to their families. That my father did not feel the same way is, as discussed in chapter 6.2, possibly an outcome of his specific individual context (Rosenfeld, Personal Communication, 17.07.2017, Schotten, Personal Communication, 18.07.2017, GB, Personal Communication, 15.12.2017 \& Bühring, Personal Communication, 31.07.2018). However, that three participants uttered regret is remarkable given that admitting errors is somewhat unusual for men since it endangers a masculinity conception mostly based on control (Cheng, 1999; Hearn, 2004; Mosse, 1998). Conceding to perhaps having made a wrong decision in the past could, therefore, indicate that, despite the fact that all interviewees more or less vehemently denied that ageing had an effect on their conception of masculinity, 
it has, in fact, led to some reflection on and subsequently also to some alterations in their understanding of masculinity.

This interpretation is further facilitated by the fact that all interviewees explicitly linked attributes such as wisdom, serenity and mentoring younger people to their older identity construction (Rosenfeld, Personal Communication, 17.07.2017, Schotten, Personal Communication, 18.07.2017, GB, Personal Communication, 15.12.2017 \& Bühring, Personal Communication, 31.07.2018). It can be assumed that these attributes did not form part of their male self-concept in younger years. This suggests that the interviewees adapted and extended their life-course narratives with elements which are indicative for older age to enable an identity construction which is more consistent with their advancing age. These were narratives which were compatible with concepts of emotional and cognitive control (Cruikshank, 2013; Woodward, 2003)- aspects which seem to still play a significant role in the identity construction in older age.

Overall, this research has found that identity in older age is still majorly determined by the hegemonic standards set by socio-cultural narratives of masculinity. Some of the parameters which define the successful performance of hegemonic masculinity, however, shift over time as individuals grow older. To establish and maintain their right to male privileges, issues such as control, domination and subordination of others become less significant. Instead, as men age, this research has demonstrated that it becomes more critical to demonstrate to oneself and others that older age only marginally impacted upon their mental and physical abilities and, most importantly, personal autonomy. The most significant factor for identity construction in older age, as this study has revealed, is the feedback received within a professional and/or public context which confirms their unremitted abilities.

Nonetheless and importantly, the participants in this study found that older age had made them softer, mfemale ore emotionally balanced and more appreciative for the little joys of life and the time left to enjoy them - an attitude which is largely at odds with masculinity constructions based on dominance and subordination of others. This significant finding suggests that men are aware of the finiteness of being and the impossibility of managing and stopping age-related decline in the long run and it hints at a potential revaluation of the factors which used to, and in many ways still, form the basis of the interviewees' male identity (Jackson, 2016).

\subsection{Reflexive Comments}

The decision to undertake a $\mathrm{PhD}$ was motivated by the wish to further my professional and personal development and by a genuine interest in gaining a better understanding of what 
getting older meant to the individual and what different perceptions of older age existed in Germany. My choice was mostly an outcome of observing the ageing process of my parents and the realisation of how deeply the changes related to ageing impacted upon their selfperception.

I had watched, particularly my Mom, majorly struggling with accepting her older self and with adapting her identity construction to this older self. As a result of her substantial physical limitations, she was increasingly unable to leave the house, and she lived a life in which I was her primary social contact which in turn led to a high dependency on me for practical issues such as shopping, cleaning or gardening as well as for emotional support and social interaction. This change of power relations from my Mom being the carer to her being cared for by me was awkward for both of us. I somewhat felt overstrained and missed how my mother used to be whilst my mother's feeling of inadequacy and insignificance affected her majorly, and she became more and more depressed and less and less easy to live with. In the last stages of her life, she moved between having an overwhelmingly bad conscience for being a burden on me and not any more being able to carry her own weight and anger about and frustration with the uncontrollability of her fate.

My father on, the other hand, seemed to have much less trouble with getting older and with being retired. Although I had had certain suspicions why that was the case I wanted to find out if his rather positive approach towards his increasing age had something to do with him being a man or if it had more to do with his social context and with his mental and physical fitness. Emerging myself in the literature as well as in the first-hand data of my four interviewees, including my father, has enabled me to find answers to some of the abovementioned questions whilst it also and unexpectedly altered my understanding of myself as well as of my socio-cultural context. In fact, during the past five years, I have increasingly become aware of the fact that my research was as much an outcome of wishing to find out more about my parents' ageing process as of getting to grips with my own increasing age as a woman.

When I reflected on my own approach towards ageing not only in my professional but also in my private context in the course of writing my dissertation it became clear to me that many of the aspects which I had found to be indicative for the identity construction of my older male participants somewhat also reflected my own approach towards ageing. First of all, there can be no doubt that I, as my interviewees, do not feel as old as my chronological age suggests. In fact, I often have to remind myself of my age explicitly and I am frequently shell shocked when I see people on TV or elsewhere my age since they seem so old to me and since I somehow cannot identify with them at all. 
I also noticed that I am, much like the interviewees, already starting to feel a certain distance to the younger generation and their way of life even though I keep telling myself that this is not the case and that I am neither prejudiced against younger people nor that I feel superior to them. Instead, my life-course narrative is based on purporting that I very much enjoy being with younger people and feel inspired by their views and lifestyle. This ambiguity with regards to the attitude of older people to younger was evident in my interviewees' accounts, too. I believe that through the contact with the younger generation and the feedback they provide, on the one hand, confirms to oneself that one does not belong on the scrap heap. Yet, on the other hand, it seems that older individuals, including myself, need to stress their extensive level of experience and resulting superiority to support a positive self-perception when becoming older.

One of the main outcomes of my interview analysis was that control as expressed in personal autonomy and meaningful activity was at the core of my interviewees' identity construction at different life stages and also in older age. I, however, kept and keep asking myself if these aspects are significant only in men's lives. When I examine myself, I have to say that I doubt this. I not only feel a strong need for controlling myself and continuously try to adjust the world around me in a way that I feel in control but I also noticed that not being active in a professional context had a negative impact on the way I feel about and look at myself. Apparently, to feel meaningful and socially visible and appreciated, it is not enough for me to take care of my pets, the house and the garden. Not even work on my $\mathrm{PhD}$, and other scientific research could really remove the feeling of being useless and insignificant. I believe this feeling to be the result of the internalisation of the master neoliberal narrative, which celebrates productivity and autonomy whilst inactivity, idleness and dependency are viewed as condemnable (DeFalco, 2016). However, although to my mind each individual who grew up in a society informed by neoliberal values feels a specific need to control oneself and to prove his or her usefulness and social value by occupying oneself meaningfully, I think that what is considered to be meaningful is mainly as a result of one's socialisation and this is evaluated differently by men and women.

At the end of my research, I feel that I have indeed gained relevant and novel insights into the life-course narratives of my interviewees and a better understanding of how they construct their identity in older age within and around the socio-cultural narratives of ageing in the west whilst also understanding my own life-course narratives better. At the same time, however, I am now more than ever before aware of the many avenues of research which could and need to be further pursued to increase our understanding of the way older people construct their identity. Such research could subsequently facilitate both the development of 
positive life-course narratives throughout one's life and fair, sustainable and equal inclusion of all older people into western societies.

\subsection{Suggestions for Future Research}

Chronological time and socio-cultural perceptions of what one should have reached, at what age, and how one should act and behave at particular life stages, exerts an enormous influence on the self-perception of older people as well as on how they are perceived in society. I, therefore, believe that questions such as 'Is there a specific time at which chronological and individual conceptions of age start to divert and if so what could this be attributed to?' or 'Are there differences in the subjective feeling of age in different cultural settings, socio-demographic groups and/or genders?' need to be asked. More empirical research aimed at answering these and related questions would support gaining insights into the subjective conception of time within one culture and across different cultures and their impact on individual and socio-cultural views of ageing.

To my mind, intergenerational relationships and social comparison upwards and downwards are of particular relevance when researching constructions of older age since intergenerational cooperation and an awareness for the mechanisms enabling a positive selfevaluation are, in my view, key in altering and adapting current conceptions of older age so that older and younger people can benefit from each other. This could support an understanding of older age which communicates that being old is not something to be feared or rejected but that it can be a fulfilling and fruitful stage of life. Within this context, it would be particularly interesting to focus on the role, intergenerational relationships and social comparison play in the self-perception of older people as well as in hegemonic narratives linked to ageing in different cultures. In addition, examining these and related topics with a higher number of people of different ages, genders and social backgrounds in the same or different countries in the occidental and/or oriental world, in group interviews or focus groups could allow for more depth and, at the same time more breadth when accessing the life-worlds of older people.

Since my interviewees clearly used to belong to a group of men which can be considered as hegemonic and whose status as hegemonic men was mostly determined by their professional activity, exploring the life-course narratives of older men who during their career belonged to more marginalised groups of men and of older women from varying socio-cultural backgrounds could markedly expand the insights gained in this project. Also, my focus on the self-perception and identity construction of older German men could be extended by capturing the experiences of older men and women in other countries and particularly within 
different cultural contexts. This could then also shed light on the question if specific nationalities such as Germans feel due to their past a particular need for control which I suspected but could for lack of comparison with other nationalities not conclusively answer. In addition, more research into the representation of older age in media outlets less in the focus - but to my mind not less powerful - of media research than films, advertising and TV programmes such as popular music and accompanying music videos, video games or U-tube videos could potentially lead to a better understanding of the socio-cultural narratives linked to older age. Lastly, there can be no doubt that me being a middle-aged female had an impact on the way the interviewees communicated their life-course narratives to me. It could garner interesting and perhaps different insights into the life-course narratives of older (German) men if they were interviewed by a man their age or younger.

The above suggestions for future research indicate some but by no means all possibilities which could be explored further to generate more insights into the themes linked to individual and socio-cultural constructions of older age and gender. Research in ageing studies has significantly increased in the past three decades. Nonetheless, I believe that given the demographic development and the fact that everybody ages, further expanding scholarly work by encouraging interdisciplinary and cross-cultural cooperation is of utmost importance. I am looking forward to continuing exploring these avenues of research and contributing to the future development of this research area. 
Addison, H. (2006). 'Must the Players Keep Young?': Early Hollywood's Cult of Youth. Cinema Journal, 45(4), 3-25.

Addison, H. (2010). 'That Younger, Fresher Woman': Old Wives for New (1918) and Hollywood's Cult of Youth. In V. B. Lipscomb \& L. Marshall (Eds.), Staging Age: The Performance of Age in Theatre, Dance, and Film (pp. 11-26). New York: Palgrave Macmillian.

Alex, L., Hammarström, A., Norberg, A., \& Lundman, B. (2008). Construction of Masculinities among Men Aged 85 and Older in the North of Sweden. Journal of Clinical Nursing, 17(4), 451-459.

Allen, J., Buzzanell, P. M., de Syon, G., D’Enbeau, S., du Pré, A., Erhart, I., . . Haridakis, P. (2015). Aging Heroes: Growing Old in Popular Culture. Lanham: Rowman \& Littlefield.

Alsemgeest, L. (2014). Family Communication About Money: Why the Taboo? Mediterranean Journal of Social Sciences, 5(16), 516-523.

Andrews, M., Squire, C., \& Tamboukou, M. (2008). Doing Narrative Research. London: Sage.

Arber, S., Davidson, K., \& Ginn, J. (2003). Gender and Ageing: Changing Roles and Relationships. Maidenhead: McGraw-Hill Education.

Argyris, C. (2002). Double-Loop Learning, Teaching, and Research. Academy of Management Learning \& Education, 1(2), 206-218.

Argyris, C., \& Schön, D. (1996). Organizational Learning Ii: Theory, Method, and Practice. Boston: Addison-Wesley.

Ayalon, L., \& Tesch-Römer, C. (2018). Contemporary Perspectives on Ageism. Berlin: Springer.

Baars, J. (2013). Critical Turns of Aging, Narrative and Time. International Journal of Ageing and Later Life, 7(2), 143-165.

Baars, J. (2015). Time in Late Modern Aging. In J. Twigg \& W. Martin (Eds.), Routledge Handbook of Cultural Gerontology. London \& New York: Routledge.

Banet-Weiser, S. (2014). "We Are All Workers" Economic Crisis, Masculinity, and the American Working Class. In D. Negra \& Y. Tasker (Eds.), Gendering the Recession: Media and Culture in an Age of Austerity (pp. 81-106). Durham: Duke University Press.

Barker, M. (2008). Analysing Discourse. In M. Pickering (Ed.), Research Methods for Cultural Studies (pp. 150-174). Edinburgh: Edinburgh University Press. 
Basit, T. (2003). Manual or Electronic? The Role of Coding in Qualitative Data Analysis. Educational Research, 45(2), 143-154.

Bauer, S., Heinemann, T., \& Lemke, T. (2017). Science and Technology Studies: Klassische Positionen Und Aktuelle Perspektiven [Science and Technology Studies: Traditional and Current Perspectives]. Frankfurt a. Main: Suhrkamp.

Bauman, Z. (2000). Modernity and the Holocaust. Ithaca: Cornell University Press.

Bauman, Z. (2013). Liquid Modernity. Hoboken: John Wiley \& Sons.

Baur, N., \& Blasius, J. (2014). Handbuch Methoden Der Empirischen Sozialforschung [Handbook of Methods for Empirical Social Research]. Berlin: Springer.

Baur, N., \& Luedtke, J. (2008). Die Soziale Konstruktion Von Männlichkeit: Hegemoniale Und Marginalisierte Männlichkeiten in Deutschland. Opladen \& Farming Hills: Barbara Budrich.

Baxter, P., \& Jack, S. (2008). Qualitative Case Study Methodology: Study Design and Implementation for Novice Researchers. The Qualitative Report, 13(4), 544-559.

Beard, J., Biggs, S., Bloom, D. E., Fried, L. P., Hogan, P. R., Kalache, A., \& Olshansky, S. J. (2012). Global Population Ageing: Peril or Promise?Retrieved from http://demographic-

challenge.com/files/downloads/6c59e8722eec82f7ffa0f1158d0f4e59/ageingbook_0 10612.pdf

Beauvoir, S. d. (1972). The Coming of Age. New York: G.P. Putnam's Sons.

Bengtson, V. L., Coleman, P. G., \& Kirkwood, T. B. (2005). The Cambridge Handbook of Age and Ageing. Cambridge: Cambridge University Press.

Berger, P. L., \& Luckmann, T. (1991). The Social Construction of Reality: A Treatise in the Sociology of Knowledge. London: Penguin.

Beynon, J. (2001). Masculinities and Culture. Buckingham: McGraw-Hill Education.

Bhaskar, R. (1975). Forms of Realism. Philosophica, 15(1), 99-127.

Bhaskar, R. (1989). The Possibility of Naturalism: A Philosophical Critique of the Contemporary Human Sciences (2. ed.). Hemel Hempstead: Harvester Wheatsheaf. Bhaskar, R. (2009). Scientific Realism and Human Emancipation. London \& New York: Routledge.

Bhaskar, R. (2013). A Realist Theory of Science. London \& New York: Routledge. Bingham, D. (1994). Acting Male: Masculinities in the Films of James Stewart, Jack Nicholson, and Clint Eastwood. New Brunswick: Rutgers University Press.

Birbili, M. (2000). Translating from One Language to Another. Social Research Update, $31(1), 1-7$.

Blaikie, A. (1999). Ageing and Popular Culture. Cambridge: Cambridge University Press. 
Blichfeldt, B. S., \& Heldbjerg, G. (2011). Why Not?: The Interviewing of Friends and Acquaintances: Department of Entrepreneurship and Relationship Management, University of Southern Denmark.

Bolin, G., \& Skogerbø, E. (2013). Age, Generation and the Media. Northern Lights: Film \& Media Studies Yearbook, 11(1), 3-14.

Bordo, S. (1999). The Male Body: A New Look at Men in Public and in Private. New York: Farrar, Straus and Giroux.

Bordo, S. (2004). Unbearable Weight: Feminism, Western Culture, and the Body. Berkeley: University of California Press.

Borutta, M., \& Verheyen, N. (2010). Die Präsenz Der Gefühle: Männlichkeit Und Emotion in Der Moderne [the Presence of Feelings: Masculinity and Emotions in Modernity]. Bielefeld: Transcript

Bourdieu, P. (1990). The Logic of Practice. Stanford: Stanford University Press.

Bourdieu, P. (2001). Masculine Domination. Stanford: Stanford University Press.

Bourdieu, P. (2011). The Forms of Capital (1986). In J. Richardson (Ed.), Handbook of Theory and Research for the Sociology of Education (pp. 241-258). Westport: Greenwood.

Bourdieu, P. (2017). Die Männliche Herrschaft [Masculine Domination] (4. ed.). Berlin: Suhrkamp Taschenbuch Wissenschaft.

Bourdieu, P. (2018). Structures, Habitus, Practices. London: Routledge.

Bourdieu, P. (December 1998). The Essence of Neoliberalism: What Is Neoliberalism?: A Programme for Destroying Collective Structures Which May Impede the Pure Market Logic: Utopia of Endless Exploitation.

Boyle, E., \& Brayton, S. (2012). Ageing Masculinities and "Muscle Work" in Hollywood Action Film: An Analysis of the Expendables. Men \& Masculinities, 15(5), 468485. doi:10.1177/1097184X12454854

Brandes, H. (2007). Hegemonic Masculinities in East and West Germany (German Democratic Republic and Federal Republic of Germany). Men and Masculinities, 10(2), 178-196.

Braun, V., \& Clarke, V. (2006). Using Thematic Analysis in Psychology. Qualitative Research in Psychology, 3(2), 77-101.

Brockmeier, J., \& Carbaugh, D. A. (2001). Narrative and Identity: Studies in Autobiography, Self and Culture. Amsterdam: John Benjamins Publishing Company. 
Brown, J. A. (2004). Gender, Sexuality, and Toughness: The Bad Girls of Action Film and Comic Books. In S. Inness (Ed.), Action Chicks: New Images of Tough Women in Popular Culture (pp. 47-74). Berlin: Springer.

Brown, T. S. (2013). West Germany and the Global Sixties: The Anti-Authoritarian Revolt, 1962-1978. Cambridge: Cambridge University Press.

Budgeon, S. (2014). The Dynamics of Gender Hegemony: Femininities, Masculinities and Social Change. Sociology, 48(2), 317-334.

Bühring, L.-N. (2017). Declining to Decline: Aged Tough Guys in the Expendables and the Expendables 2. Journal of Extreme Anthropology, 1(3), 1-20.

Burke, P. J. (2011). Masculinity, Subjectivity and Neoliberalism in Men's Accounts of Migration and Higher Educational Participation. Gender and Education, 23(2), 169-184.

Burr, V. (2006). An Introduction to Social Constructionism. Abingdon: Routledge.

Butler, J. (2011). Gender Trouble: Feminism and the Subversion of Identity. Abingdon: Routledge.

Calasanti, T., \& King, N. (2005). Firming the Floppy Penis: Age, Class, and Gender Relations in the Lives of Old Men. Men and Masculinities, 8(1), 3-23. doi:10.1177/1097184X04268799

Calasanti, T., \& King, N. (2015). Intersectionality and Age. In J. Twigg \& W. Martin (Eds.), Routledge Handbook of Cultural Gerontology (pp. 193-200). London \& New York: Routledge.

Calasanti, T., \& Slevin, K. (2001). Gender, Social Inequalities, and Aging. Lanham: Rowman Altamira.

Capstick, A., \& Clegg, D. (2013). Behind the Stiff Upper Lip: War Narratives of Older Men with Dementia. Journal of War \& Culture Studies, 6(3), 239-254. doi:10.1179/1752627213Z.00000000021

Carroll, M., \& Bartlett, H. (2015). Ageing Well across Cultures. In J. Twigg \& W. Martin (Eds.), Routledge Handbook of Cultural Gerontology (pp. 285-293). London \& New York: Routledge.

Chambers, C. (2005). Masculine Domination, Radical Feminism and Change. Feminist Theory, 6(3), 325-346.

Chang, H.-J., \& Aldred, J. (2014). After the Crash, We Need a Revolution in the Way We Teach Economics. The Observer.

Cheng, C. (1999). Marginalized Masculinities and Hegemonic Masculinity: An Introduction. The Journal of Men's Studies, 7(3), 295-315. 
Chivers, S. (2011). The Silvering Screen: Old Age and Disability in Cinema. Toronto, Buffalo \& London: University of Toronto Press.

Chomsky, N. (1999). Profit over People: Neoliberalism and Global Order. New York: Seven Stories Press.

Clarke, L. H. (2010). Facing Age: Women Growing Older in Anti-Aging Culture. Lanham: Rowman \& Littlefield Publishers.

Clarke, L. H., \& Bennett, E. V. (2015). Gender, Ageing and Appearance. In J. Twigg \& W. Martin (Eds.), Routledge Handbook of Cultural Gerontology (pp. 133-140).

London \& New York: Routledge.

Clover, C. J. (1993). Men, Women, and Chain Saws: Gender in the Modern Horror Film. Princeton: Princeton University Press.

Collier, A. (1994). Critical Realism: An Introduction to Roy Bhaskar's Philosophy. London \& New York: Verso.

Collinson, D. L. (2010). Managing the Shopfloor: Subjectivity, Masculinity and Workplace Culture. Berlin: Walter de Gruyter.

Collopy, B. (2015). The Country for Old Men: The Rise of the Ageing Action Hero in Film. Retrieved from https://burningpapertigers.wordpress.com/2015/04/23/thecountry-for-old-men-the-rise-of-the-ageing-action-hero-in-film/

Colvin, S., \& Davies, P. J. (2008). Masculinities in German Culture. Rochester: Camden House.

Comstock, D. (1982). A Method for Critical Research. In E. Bredo \& W. Feinberg (Eds.), Knowledge and Values in Social and Educational Research (pp. 370-390).

Philadelphia: Temple University Press. Retrieved from

http://goodliffe.byu.edu/301/protect/comstock.pdf.

Connell, R. (1993). The Big Picture: Masculinities in Recent World History. Theory and Society, 22(5), 597-623.

Connell, R. (1998). Masculinities and Globalization. Men and Masculinities, 1(1), 3-23.

Connell, R. (2005). Masculinities (2 ed.). Cambridge: Polity.

Connell, R. (2009). A Thousand Miles from Kind: Men, Masculinities and Modern Institutions. The Journal of Men's Studies, 16(3), 237-252.

Connell, R. (2014). The Study of Masculinities. Qualitative Research Journal, 14(1), 5-15.

Connell, R., \& Messerschmidt, J. W. (2005). Hegemonic Masculinity Rethinking the Concept. Gender \& Society, 19(6), 829-859.

Cooper, H., \& Hedges, L. V. (2009). Research Synthesis as a Scientific Process. In H. Cooper, L. V. Hedges, \& J. C. Valentine (Eds.), The Handbook of Research Synthesis and Meta-Analysis (2 ed., pp. 3-17). New York: Russell Sage Foundation. 
Corcoran, K., Crusius, J., \& Mussweiler, T. (2011). Social Comparison: Motives, Standards, and Mechanisms. In D. Chadee (Ed.), Theories in Social Psychology (pp. 119-139). Oxford: Wiley-Blackwell.

Cornwall, A., Karioris, F. G., \& Lindisfarne, N. (2016). Masculinities under Neoliberalism. London: Zed Books.

Coston, B. M., \& Kimmel, M. (2012). Seeing Privilege Where It Isn't: Marginalized Masculinities and the Intersectionality of Privilege. Journal of Social Issues, 68(1), 97-111.

Cramer, R. (2015). German Ordnung: A Semantic and Ethnopragmatic Analysis of a Core Cultural Value. International Journal of Language and Culture, 2(2), 269-293.

Creswell, J. W. (2009). Research Design: Qualitative, Quantitative, and Mixed Methods Approaches (3rd Ed.). Thousand Oaks: Sage.

Creswell, J. W., \& Miller, D. L. (2000). Determining Validity in Qualitative Inquiry. Theory into Practice, 39(3), 124-130.

Crosnoe, R., \& Elder, G. H., Jr. (2002). Successful Adaptation in the Later Years: A Life Course Approach to Aging. Social Psychology Quarterly, 65(4), 309-328. doi: $10.2307 / 3090105$

Crouthamel, J. (2008). Male Sexuality and Psychological Trauma: Soldiers and Sexual Disorder in World War I and Weimar Germany. Journal of the History of Sexuality, $17(1), 60-84$.

Cruikshank, M. (2013). Learning to Be Old: Gender, Culture, and Aging. Lanham, Boulder, New York, Toronto, Plymouth: Rowman \& Littlefield.

Cullum-Swan, B., \& Manning, P. (1994). Narrative, Content, and Semiotic Analysis. In N. K. Denzin \& Y. S. Lincoln (Eds.), Handbook of Qualitative Research (pp. 463478). Newbury Park: Sage.

Danermark, B., Ekstrom, M., \& Jakobsen, L. (2005). Explaining Society: An Introduction to Critical Realism in the Social Sciences. Oxon \& New York: Routledge.

Davenport, K. (2008). Gender and Aging: An Investigation of Television's Infatuation with Youth and Beauty. (Honors Senior Thesis Senior Thesis), Boston University, Boston. Retrieved from http://88.198.249.35/d/Gender-and-Communication.pdf Davey, J., \& Glasgow, K. (2006). Positive Ageing - a Critical Analysis. Policy Quarterly, 2(4), 21-27.

de Medeiros, K. (2005). The Complementary Self: Multiple Perspectives on the Aging Person. Journal of Ageing Studies, 19(1), 1-13. doi:10.1016/j.jaging.2004.02.001 de Medeiros, K. (2016). Narrative Gerontology: Countering the Master Narratives of Aging. Narrative Works, 6(1), 63-81. 
de Medeiros, K., \& Black, H. (2015). Suffering and Pain in Old Age. In J. Twigg \& W. Martin (Eds.), The Routledge Handbook of Cultural Gerontology. (pp. 181-187). London and New York: Routledge.

de Pater, I. E., Judge, T. A., \& Scott, B. A. (2014). Age, Gender, and Compensation: A Study of Hollywood Movie Stars. Journal of Management Inquiry, 23(4), 407-420. DeFalco, A. (2016). In Praise of Idleness: Aging and the Morality of Inactivity. Cultural Critique, 92(Winter 2016), 84-113.

Denham, M. J. (1984). The Ethics of Research in the Elderly. Age and Ageing, 13(6), 321327. doi:10.1093/ageing/13.6.321

Denzin, N. K. (2001). The Reflexive Interview and a Performative Social Science. Qualitative Research, 1(1), 23-46.

Denzin, N. K. (2006). Sociological Methods: A Sourcebook. New York: Aldine Transaction.

DiCicco-Bloom, B., \& Crabtree, B. F. (2006). The Qualitative Research Interview. Medical Education, 40(4), 314-321.

Dobbins, J. F. (2003). America's Role in Nation-Building: From Germany to Iraq. Survival, 45(4), 87-110.

Dolan, J. (2013). Firm and Hard: Gender, Old Age and Hollywood's Gaze. In J. I. PrietoArranz, P. Bastida-Rodríguez, M. Fernández-Morales, \& C. Suárez-Gómez (Eds.), De-Centring Cultural Studies: Past, Present and Future of Popular Culture (pp. 217-246). Newcastle upon Tyne: Cambridge Scholars Publishing.

Dolan, J. (2018). Contemporary Cinema And'old Age': Gender and the Silvering of Stardom. London: Palgrave Macmillan.

Dolan, J., \& Tincknell, E. (2012). Aging Femininities: Troubling Representations.

Newcastle upon Tyne: Cambridge Scholars Publishing.

Donnar, G. (2016). Narratives of Cultural and Professional Redundancy: Ageing Action Stardom and the 'Geri-Action'film. Communication, Politics \& Culture, 49(1), 118.

Doucet, A., \& Mauthner, N. (2006). Feminist Methodologies and Epistemology. In C. D. Bryant \& D. L. Peck (Eds.), Handbook of 21st Century Sociology (pp. 36-42). Newbury Park: Sage.

Duggan, L. (2002). The New Homonormativity: The Sexual Politics of Neoliberalism. In R. C. D. D. Nelson (Ed.), Materializing Democracy: Toward a Revitalized Cultural Politics (pp. 175-194). Durham: Duke University Press. 
Dustmann, C., Fitzenberger, B., Schönberg, U., \& Spitz-Oener, A. (2014). From Sick Man of Europe to Economic Superstar: Germany's Resurgent Economy. Journal of Economic Perspectives, 28(1), 167-188.

Dutt, R. (2014). Behind the Curtain: Women's Representations in Contemporary Hollywood. (Masters), London School of Economics and Political Science, London.

Dyer, R. (2013). Heavenly Bodies: Film Stars and Society. Abingdon: Routledge.

Eckersley, R. (2006). Is Modern Western Culture a Health Hazard? International Journal of Epidemiology, 35(2), 252-258.

Edley, N., \& Wetherell, M. (1995). Men in Perspective. Hemel Hempstead: Pearson Education Limited.

Edwards, T. (2004). Cultures of Masculinity. London: Routledge.

Elder-Vass, D. (2012). Towards a Realist Social Constructionism. Sociologia, Problemas e Práticas, 70(2012), 5-24.

Esipova, N., Ray, J., \& Pugliese, A. (2017). How the World Views Migration.Retrieved from https://publications.iom.int/system/files/how the world_gallup.pdf

Ettelson, T. R. (2002). The Nazi New Man: Embodying Masculinity and Regulating Sexuality in the Sa and Ss, 1930--1939. (PhD Dissertation), University of Michigan, Ann Arbor.

Evans, R. (2015). Society and Politics in Wilhelmine Germany (Routledge Revivals). Abingdon: Routledge.

Fairclough-Isaacs, K. (2015). Celebrity Culture and Ageing. In J. Twigg \& W. Martin (Eds.), The Routledge Handbook of Cultural Gerontology (pp. 361-368). London and New York.

Fairclough, K. (2012). Nothing Less Than Perfect: Female Celebrity, Ageing and HyperScrutiny in the Gossip Industry. Celebrity Studies, 3(1), 90-103.

Fausto-Sterling, A. (1997). How to Build a Man. In V. A. Rosario (Ed.), Science and Homosexualities (pp. 219--225). Abingdon: Routledge.

Fausto-Sterling, A. (2000). Sexing the Body: Gender Politics and the Construction of Sexuality. New York: Basic Books.

Fausto-Sterling, A. (2008). Myths of Gender: Biological Theories About Women and Men. New York: Basic Books.

Fay, B. (1987). Critical Social Science: Liberation and Its Limits. Cambridge and Oxford: Polity.

Fay, B. (1996). Contemporary Philosophy of Social Science: A Multicultural Approach. Oxford: Blackwell 
Fay, B. (2014). Social Theory and Political Practice (Rle Social Theory). Abingdon: Routledge.

Feasey, R. (2011). Mature Masculinity and the Ageing Action Hero. Groniek, 190(2011), 507-520.

Featherstone, M. (1991). The Body in Consumer Culture. In M. Featherstone, M.

Hepworth, B. S. Turner, M. Hepworth, \& B. S. Turner (Eds.), The Body: Social Process and Cultural Theory (pp. 170-196). Thousand Oaks: Sage Publications.

Featherstone, M. (1995). Undoing Culture: Globalization, Postmodernism and Identity.

Newcastle upon Tyne: Sage.

Featherstone, M., \& Hepworth, M. (2005). Images of Ageing: Cultural Representations of Later Life. In V. L. Bengtson, P. G. Coleman, \& T. B. Kirkwood (Eds.), Cambridge Handbook of Age and Ageing (pp. 354-362). Cambridge: Cambridge University Press.

Featherstone, M., \& Wernick, A. (1995). Images of Aging: Cultural Representations of Later Life. London \& New York: Taylor \& Francis.

Feinberg, L. (1996). Transgender Warriors: Making History from Joan of Arc to Dennis Rodman. Boston: Beacon Press.

Festinger, L. (1954). A Theory of Social Comparison Processes. Human Relations, 7(2), $117-140$.

Finanzen100. (16.07.2018). Angst Vor Altersarmut Übertrieben? Die Unbequeme Wahrheit Über Unsere Rente [Fear of Old Age Poverty Exaggerated? The Unpleasant Truth About Our Pension].

Fitterer, S. (2013). The Emergence of Gender Role Anxieties in the Weimar Republic. The Corvette, 2(1), 28-39.

Fletcher, J. (1997). Violence and Civilization: An Introduction to the Work of Norbert Elias. Hoboken: John Wiley \& Sons.

Flick, U. (2009). An Introduction to Qualitative Research. London, Thousand Oaks, New Delhi \& Singapore: Sage.

Flood, M. (1997). Doing Research on Men and as Men: Politics and Problems. Paper presented at the Masculinities: Renegotiating Genders, Wollongong.

Foucault, M. (1978). Dispositive Der Macht: Über Sexualität, Wissen Und Wahrheit [Dispositif of Power: About Sexuality, Knowledge and Truth]. Leipzig: Merve.

Foucault, M. (2000). Ethics Subjectivity and Truth; the Essential Works of Michael Foucault, 1954-1984 (J. D. Faubion Ed.). New York: New Press. 
Foucault, M. (2003). Sexualität Und Wahrheit. Der Wille Zum Wissen, Erster Band [Sexuality and Truth. The Will to Know, First Volume]. Frankfurt a. Main: Suhrkamp.

Foucault, M., Davidson, A. I., \& Burchell, G. (2008). The Birth of Biopolitics: Lectures at the Collège De France, 1978-1979. Berlin: Springer.

Fulbrook, M. (2004). A Concise History of Germany. Cambridge: Cambridge University Press.

Fung, H. (2013). Aging in Culture. The Gerontologist, 53(3), 369-377. doi:10.1093/geront/gnt024

Gabbard, K., \& Luhr, W. (2008). Screening Genders. New Brunswick: Rutgers University Press.

Gallagher, M. (2006). Action Figures: Men, Action Films and Contemporary Adventure Narratives. New York: Palgrave Macmillan.

Gardiner, S. L. (2004). Masculinity, War, and Refusal: Vicissitudes of German Manhood before and after the Cold War. (PhD PhD Dissertation), Cornell University, Cornell

Geertz, C. (2008). Thick Description: Toward an Interpretive Theory of Culture. In T. S. Oakes \& P. L. Price (Eds.), The Cultural Geography Reader (pp. 41-51). London: Routledge.

Gergen, K. J., \& Gergen, M. M. (2000). The New Aging: Self Construction and Social Values. In K. W. Schaie (Ed.), Social Structures and Aging (pp. 281-306). New York: Springer.

Gill, R. (2008). Culture and Subjectivity in Neoliberal and Postfeminist Times.

Subjectivity, 2(1), 432-445.

Gill, R., Hamad, H., Kauser, M., Negra, D., \& Roshini, N. (2016). Intergenerational Feminism and Media: A Roundtable. Feminist Media Studies, 16(4), 726-736.

Gilleard, C. J., \& Higgs, P. (2000). Cultures of Ageing: Self, Citizen, and the Body. London: Pearson Education.

Goffman, E. (2009). Stigma: Notes on the Management of Spoiled Identity. New York: Simon and Schuster.

Golafshani, N. (2003). Understanding Reliability and Validity in Qualitative Research. The Qualitative Report, 8(4), 597-606.

Google Images. (2017). Older People. Retrieved from

https://www.google.com/search?q=older+people+images\&client=firefox-bd\&sxsrf=ACYBGNQWqnKESLc7PG0eOX5qhbFKxI2IyQ:1578464084463\&sour

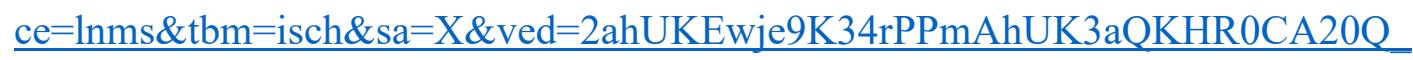


Gotto, B., Seefried, Elke. (2017). Männer Mit “Makel”: Männlichkeiten Und

Gesellschaftlicher Wandel in Der Frühen Bundesrepublik [ 'Flawed' Men and

Social Change in the Early Federal Republic of Germany]. Oldenburg: De Gruyter.

Gravagne, P. H. (2013). The Becoming of Age - Cinematic Visions of Mind, Body, and

Identity in Later Life. Jefferson \& London: McFarland \& Company.

Gray, M. W. (2000). Productive Men, Reproductive Women: The Agrarian Household and the Emergence of Separate Spheres During the German Enlightenment. Oxford \& New York: Berghahn Books.

Greig, A., Kimmel, M., \& Lang, J. (2000). Men, Masculinities and Development:

Broadening Our Work Towards Gender Equality. New York: United Nations Development Programme.

Grenier, A. (2012). Transitions and the Lifecourse: Challenging the Constructions of 'Growing Old'. Chicago and Bristol: Policy Press.

Greven, D. (2010). Manhood in Hollywood from Bush to Bush. Austin: University of Texas Press.

Griffin, P. (2005). Neoliberal Economic Discourses and Hegemonic Masculinity (Ies):

Masculine Hegemony (Dis) Embodied. IPEG Papers in Global Political Economy(19), 1-23.

Grix, J. (2010). The Foundations of Research. London: Palgrave Macmillan.

Grossmann, I., Karasawa, M., Izumi, S., Na, J., Varnum, M. E., Kitayama, S., \& Nisbett,

R. E. (2012). Aging and Wisdom: Culture Matters. Psychological Science, 23(10), 1059-1066.

Guba, E. G., \& Lincoln, Y. S. (1994). Competing Paradigms in Qualitative Research. In N.

K. Denzin \& Y. S. Lincoln (Eds.), Handbook of Qualitative Research (pp. 105-

117). Thousand Oaks: Sage Publications.

Guba, E. G., \& Lincoln, Y. S. (2005). Paradigmatic Controversies, Contradictions, and Emerging Confluences. In N. K. Denzin \& Y. S. Lincoln (Eds.), The Sage Handbook of Qualitative Research (3. ed., pp. 191-215). Thousand Oaks: Sage Publications.

Guillemin, M., \& Gillam, L. (2004). Ethics, Reflexivity, and "Ethically Important Moments" in Research. Qualitative Inquiry, 10(2), 261-280.

Gullette Morganroth, M. (2003). From Life Storytelling to Age Autobiography. Journal of Aging Studies, 17(1), 101-111.

Gullette Morganroth, M. (2004). Aged by Culture. Chicago \& London: University of Chicago Press. 
Gullette Morganroth, M. (2008). What Exactly Has Age Got to Do with It? My Life in

Critical Age Studies. Journal of Aging Studies, 22(2), 189-195.

doi:http://dx.doi.org/10.1016/j.jaging.2007.12.004

Gullette Morganroth, M. (2011). Agewise: Fighting the New Ageism in America. Chicago:

University of Chicago Press.

Hall, S. (2000). Paths to Anelpis: 1: Dimorphic Violence and the Pseudo-Pacification

Process. Parallax, 6(2), 36-53.

Hall, S. (2002). Daubing the Drudges of Fury: Men, Violence and the Piety of the

'Hegemonic Masculinity' Thesis. Theoretical Criminology, 6(1), 35-61.

Hall, S. (2015). What Is Criminology About? The Study of Harm, Special Liberty and

Pseudo-Pacification in Late-Capitalism's Libidinal Economy. In R. Lippens \& D.

Crewe (Eds.), What Is Criminology About? Philosophical Reflections (pp. 1-29).

Abingdon: Routledge.

Hall, S., \& Winlow, S. (2015). Revitalizing Criminological Theory: Towards a New Ultra-

Realism. Abingdon: Routledge.

Hamad, H. (2013). Postfeminism and Paternity in Contemporary Us Film: Framing

Fatherhood. Abingdon: Routledge.

Hammersley, M. (2007). The Issue of Quality in Qualitative Research. International

Journal of Research \& Method in Education, 30(3), 287-305.

doi:10.1080/17437270701614782

Haraway, D. (1988). Situated Knowledges: The Science Question in Feminism and the

Privilege of Partial Perspective. Feminist Studies, 14(3), 575-599.

Haraway, D. (2013). Simians, Cyborgs, and Women: The Reinvention of Nature.

Abingdon: Routledge.

Harding, S. (1992). Rethinking Standpoint Epistemology: What Is " Strong Objectivity?".

The Centennial Review, 36(3), 437-470.

Harvey, D. (2007a). A Brief History of Neoliberalism. Oxford: Oxford University Press.

Harvey, D. (2007b). Neoliberalism as Creative Destruction. The Annals of the American Academy of Political and Social Science, 610(1), 21-44.

Haustein, T., \& Mischke, J. (2011). In the Spotlight: Older People in Germany and the E.U.Retrieved from Wiesbaden: https://www.destatis.de/EN/Themes/SocietyEnvironment/Population/Current-Population/Publications/Downloads-Current$\underline{\text { Population/brochure-older-people-eu-0010021169004.pdf?_ blob=publicationFile }}$

Hearn, J. (1995). Imaging the Aging of Men. In M. Featherstone \& A. Wernick (Eds.), Images of Aging: Cultural Representations of Later Life (pp. 97-115). London \& New York: Francis \& Taylor. 
Hearn, J. (2004). From Hegemonic Masculinity to the Hegemony of Men. Feminist Theory, 5(1), 49-72. doi:10.1177/1464700104040813

Hearn, J. (2014). On Men, Organizations and Intersectionality: Personal, Working, Political and Theoretical Reflections (or How Organization Studies Met Profeminism). Equality, Diversity and Inclusion: An International Journal, 33(5), 414-428. doi:doi:10.1108/EDI-07-2013-0051

Hearn, J., \& Sandberg, L. (2009). Older Men, Ageing and Power: Masculinities Theory and Alternative Spatialised Theoretical Perspectives. Sextant: revue du Groupe interdisciplinaire d'etudes sur les femmes et le genre, 27(2009), 147-163.

Heron, T. (2008). Globalization, Neoliberalism and the Exercise of Human Agency. International Journal of Politics, Culture, and Society, 20(1-4), 85-101.

Hills, E. (1999). From'figurative Males' to Action Heroines: Further Thoughts on Active Women in the Cinema. Screen, 40(1), 38-50.

Hirose, A., \& Pih, K. K. (2010). Men Who Strike and Men Who Submit: Hegemonic and Marginalized Masculinities in Mixed Martial Arts. Men and Masculinities, 13(2), 190-209. doi:10.1177/1097184X09344417

Hofer, B. K. (2000). Dimensionality and Disciplinary Differences in Personal Epistemology. Contemporary Educational Psychology, 25(4), 378-405.

Hofer, B. K., \& Pintrich, P. R. (1997). The Development of Epistemological Theories:

Beliefs About Knowledge and Knowing and Their Relation to Learning. Review of Educational Research, 67(1), 88-140.

Hoffmann, E., Gordo, L. R., Nowossadeck, S., Simonson, J., \& Tesch-Römer, C. (2015).

Living Situations of Older People in Germany.Retrieved from Berlin:

https://www.dza.de/fileadmin/dza/publications/Fact Sheet Lebenssituation \% $\%$ C3\% 841terer_DZA_05-2017_EN.pdf

Hofstede, G. (1983). National Cultures in Four Dimensions: A Research-Based Theory of Cultural Differences among Nations. International Studies of Management \& Organization, 13(1-2), 46-74.

Hofstede, G. (2011). Dimensionalizing Cultures: The Hofstede Model in Context. Online Readings in Psychology and Culture, 2(1), 1-8.

Hogan, S. (2016). “Age Is Just a Number, Init?”: Interrogating Perceptions of Age and Women within Social Gerontology. Women's Studies, 45(1), 57-77. doi:10.1080/00497878.2015.1040548

Hogan, S., \& Warren, L. (2012). Dealing with Complexity in Research Processes and Findings: How Do Older Women Negotiate and Challenge Images of Aging? Journal of Women \& Aging, 24(4), 329-350. doi:10.1080/08952841.2012.708589 
Holmlund, C. (2002). Impossible Bodies: Femininity and Masculinity at the Movies.

London: Routledge.

Holmlund, C. (2010). Celebrity, Ageing and Jackie Chan: Middle-Aged Asian in Transnational Action. Celebrity Studies, 1(1), 96 - 112.

Holt, D. B., \& Thompson, C. J. (2004). Man - of - Action Heroes: The Pursuit of Heroic Masculinity in Everyday Consumption. Journal of Consumer Research, 31(2), 425440. doi:10.1086/422120

Horkheimer, M., \& Adorno, T. W. (2001). The Culture Industry: Enlightenment as Mass Deception. In G. D. D. M. Kellner (Ed.), Media and Cultural Studies: Keyworks (pp. 41-72). Malden \& Oxford: Blackwell.

Horkheimer, M., \& Adorno, T. W. (2002). Dialectic of Enlightenment: Philosophical Fragments. Stanford: Stanford University Press.

Hughes, J. (2005). Lessons in Your Rucksack: The Complete Tefl Survival Guide. n.p.: Modern English Publishing.

Hughes, P. (2014). The Expendables 3 [Feature Film]. U.S.: Lionsgate.

Hulls, A. (2013, 30.01.2013). What's Behind Hollywood's Obsession with Old Man Action Heroes? Flavorwire.

Inness, S. (2004). Action Chicks: New Images of Tough Women in Popular Culture. Berlin: Springer.

Jackson, D. (2016). Exploring Aging Masculinities: The Body, Sexuality and Social Lives. London \& New York: Palgrave MacMillan.

Jeffords, S. (1994). Hard Bodies: Hollywood Masculinity in the Reagan Era. New Brunswick: Rutgers University Press.

Jeffords, S. (1998). The" Remasculinization" of Germany in the 1950s: Discussion. Signs: Journal of Women in Culture and Society, 24(1), 163-169.

Jennings, R. (2015). Popular Music and Ageing. In J. Twigg \& W. Martin (Eds.), The Routledge Handbook of Cultural Gerontology (pp. 77-84). London \& New York: Routledge.

Jennings, R., \& Krainitzki, E. (2015). 'Call the Celebrity': Voicing the Experience of Women and Ageing through the Distinctive Vocal Presence of Vanessa Redgrave. In D. Jermyn \& S. Holmes (Eds.), Women, Celebrity and Cultures of Ageing Freeze Frame (pp. 178-196). Houndmills, Basingstoke, \& New York: Palgrave Macmillan.

Jennings, R., \& Krainitzki, E. (unpublished). 'Growing Old Disgracefully' - Notions of Subculture and Counter Culture Come of Age. 
Jennings, R., \& Oro-Piqueras, M. (2016). Heroine and/or Caricature? The Older Woman in Desperate Housewives. In M. Oró-Piqueras \& A. Wohlmann (Eds.), Serializing Age: Aging and Old Age in Tv Series. Aging Studies, Vii (pp. 71-89). Bielefeld: transcript.

Jermyn, D., \& Holmes, S. (2015). Women, Celebrity and Cultures of Ageing: Freeze

Frame. Houndmills, Basingstoke, \& New York: Palgrave Macmillan.

Johnson, B. D. (11.02.2013). You Can Stay in Hollywood - Just Act Your Age. Retrieved from https://www.macleans.ca/culture/movies/you-can-stay-just-act-your-age/

Jolanki, O. H. (2009). Agency in Talk About Old Age and Health. Journal of Aging Studies, 23(4), 215-226. doi:http://dx.doi.org/10.1016/j.jaging.2007.12.020 Jönson, H. (2012). We Will Be Different! Ageism and the Temporal Construction of Old Age. The Gerontologist, 53(2), 198-204.

Joy, S., \& Kolb, D. A. (2009). Are There Cultural Differences in Learning Style? International Journal of Intercultural Relations, 33(1), 69-85.

Joyce, K., Loe, M., \& Diamond-Brown, L. (2015). Science, Technology and Aging. In J. T. W. Martin (Ed.), Routledge Handbook of Cultural Gerontology. (pp. 157-165). London \& New York: Routledge.

Junker, D., Gassert, P., Mausbach, W., \& Morris, D. B. (2004). The United States and Germany in the Era of the Cold War, 1945-1990: A Handbook. Cambridge: Cambridge University Press.

Katz, E., Blumler, J. G., \& Gurevitch, M. (1973). Uses and Gratifications Research. Public Opinion Quarterly, 509-523.

Katz, S. (2015). Ageing, Risk and the Falling Body. In J. Twigg \& W. Martin (Eds.), Routledge Handbook of Cultural Gerontology (pp. 165-172). London \& New York: Routledge.

Katz, S., \& Calasanti, T. (2014). Critical Perspectives on Successful Aging: Does It “Appeal More Than It Illuminates"? The Gerontologist, 55(1), 26-33.

Katz, S., \& Marshall, B. (2003). New Sex for Old: Lifestyle, Consumerism, and the Ethics of Aging Well. Journal of Aging Studies, 17(1), 3-16. doi:https://doi.org/10.1016/S0890-4065(02)00086-5

Kauffman, M., \& Morris, H. J. (2015, 2016, 2017, 2018). Grace and Frankie (Season 1-4). USA: Netflix.

Kessel, M. (2003). The 'Whole Man': The Longing for a Masculine World in NineteenthCentury Germany. Gender \& History, 15(1), 1-31. doi:10.1111/1468-0424.00287

Kessler, E.-M. (2009). Altersbilder in Den Medien: Wirklichkeit Oder Illusion? [Imgages of Ageing: Reality or Illusion?]. In B. Schorb, A. Hartung, \& W. Reißmann (Eds.), 
Medien Und Höheres Lebensalter [Media and Higher Age] (pp. 146-156). Berlin: Springer.

Kessler, S. J. (1998). Lessons from the Intersexed. New Brunswick: Rutgers University Press.

Kiesewetter, H. (1996). Industrielle Revolution in Deutschland: 1815-1914. [Industrial Revolution in Germany: 1815-1914] (3. ed.). Frankfurt a. Main: Suhrkamp.

Kimmel, M. (2001). Global Masculinities: Restoration and Resistance. In R. Pease (Ed.), A Men's World?: Changing Men's Practices in a Globalized World (pp. 21-37).

London: Zed Press.

Kimmel, M. (2012). Manhood in America: A Cultural History (3 ed.). New York: Oxford University Press.

King, N. (2007). Old Cops: Occupational Aging in a Film Genre Staging Age:

Performance of Age in Theatre, Dance, and Film (pp. 57-81). New York: Palgrave Macmillan.

Kinsella, E. A. (2007). Embodied Reflection and the Epistemology of Reflective Practice. Journal of Philosophy of Education, 41(3), 395-409. doi:10.1111/j.14679752.2007.00574.x

Koivisto, J., Thomas, P., \& Aslama, M. (2008). Mapping Communication and Media Research: Paradigms, Institutions, Challenges (9521050152).Retrieved from Helsinki: http://www.helsinki.fi/crc/Julkaisut/Koivisto-ThomasPDF.pdf

Koro-Ljungberg, M. (2008). Validity and Validation in the Making in the Context of Qualitative Research. Qualitative Health Research, 18(7), 983-989. doi:10.1177/1049732308318039

Kotter-Gruhn, D., \& Hess, T. M. (2012). The Impact of Age Stereotypes on SelfPerceptions of Aging across the Adult Lifespan. The Journals of Gerontology. Series B, Psychological Sciences and Social Sciences., 67(5), 563-571. doi:10.1093/geronb/gbr153

Kotz, M. D. (2002). Globalization and Neoliberalism. Rethinking Marxism, 14(2), 64-79. Koulouriotis, J. (2011). Ethical Considerations in Conducting Research with Non-Native Speakers of English. TESL Canada Journal, 28(Special Issue 5), 1-15.

Krainitzki, E. (2014). Judi Dench's Age-Inappropriateness and the Role of M: Challenging Normative Temporality. Journal of Aging Studies, 29(April 2014), 32-40.

Kudo, S., Mutisya, E., \& Nagao, M. (2015). Population Aging: An Emerging Research Agenda for Sustainable Development. Social Sciences, 4(4), 940-966.

Kuhn, T. S. (1970). The Structure of Scientific Revolutions (2. ed.). Chicago: Univiversity of Chicago 
Kühne, T. (2009). Geschichte Der Männlichkeiten (Historische Einführungen 5) [a History of Masculinity; Historical Introduction 5]. Frankfurt a. Main: Campus.

Kühne, T. (2010). Zärtlichkeit Und Zynismus. Militärische Vergemeinschaftung 19181945 [Affectionateness and Cynicism. Military Collectivisation 1918-1945]. In M. Borutta \& N. Verheyen (Eds.), Die Präsenz Der Gefühle, Männlichkeit Und Emotion Im 19. Und 20. Jahrhundert [the Presence of Feelings, Masculinity and Emotions in the $19^{\text {th }}$ and $20^{\text {th }}$ Century] (pp. 179-202). Bielefeld: transcript.

Kuldova, T. (2014). Designing Hypermuscular Neo-Aristocracy: Of Kings, Gangsters and Muscles in Indian Cinema, Fashion and Politics. Film, Fashion \& Consumption, $3(2), 149-156$.

Kuldova, T. (2016). Luxury Indian Fashion: A Social Critique. London: Bloomsbury Publishing.

Laceulle, H., \& Baars, J. (2014). Self-Realization and Cultural Narratives About Later Life. Journal of Aging Studies, 31(December), 34-44. doi:http://dx.doi.org/10.1016/j.jaging.2014.08.005

Lamb, S., Robbins-Ruszkowski, J., Corwin, A., Calasanti, T., \& King, N. (2017). Successful Aging as a Contemporary Obsession: Global Perspectives. New Brunswick, Camden, and Newark, New Jersey, and London: Rutgers University Press.

Larkin, P. J., Dierckx de Casterlé, B., \& Schotsmans, P. (2007). Multilingual Translation Issues in Qualitative Research: Reflections on a Metaphorical Process. Qualitative Health Research, 17(4), 468-476.

Lauzen, M. M. (2015). It's a Man's (Celluloid) World: On-Screen Representations of Female Characters in the Top 100 Films of 2014.Retrieved from https://www.google.com/url? sa=t\&rct=j\&q=\&esrc=s\&source=web\&cd=\&cad=rja $\underline{\text { \&uact }=8 \& \text { ved=2ahUKEwjdp_W 9oDrAhUDSBUIHVXNCboQFjABegQIARAB }}$ \&url=https $\% 3 \mathrm{~A} \% 2 \mathrm{~F} \% 2 \mathrm{Fw}$ omenintvfilm.sdsu.edu $\% 2 \mathrm{Ffiles} \% 2 \mathrm{~F} 2013$ It $\% 2527 \mathrm{~s}$ a Man\%2527s_World_Report.pdf\&usg=AOvVaw2V24-1vo2e75BEc0dBpzsz

Law, R., Campbell, H. R., \& Dolan, J. C. (1999). Masculinities in Aotearoa/New Zealand. Auckland: Dunmore Press.

Lawson, T. (1999). Feminism, Realism, and Universalism. Feminist Economics, 5(2), 25 59.

Lawson, T. (2003). Theorizing Ontology. Feminist Economics, 9(1), 161-169.

Lazar, D., \& Mauch, C. (2004). The United States and Germany in the Era of the Cold War, 1945-1990: A Handbook (Vol. 1). Cambridge: Cambridge University Press. 
Leek, C. (2016). Masculinities under Neoliberalism. Culture, Society and Masculinities, $8(2), 176$.

Lehman, P. (2001). Masculinity: Bodies, Movies, Culture. New York: Routledge.

Lemke, T. (2014). Gouvernementalität [Governmentality]. In C. Kammler, R. Parr, \& U. J. Schneider (Eds.), Foucault-Handbuch [Foucault: A Handbook] (pp. 260-263). Berlin: Springer.

Lennard, D. (2014). Too Old for This Shit?: On Ageing Tough Guys. In I. Whelehan \& J. Gwynne (Eds.), Ageing, Popular Culture and Contemporary Feminism (pp. 93107). New York: Palgrave MacMillan.

Levsen, S. (2008). Constructing Elite Identities: University Students, Military Masculinity and the Consequences of the Great War in Britain and Germany. Past \& Present, 198(1), 147-183. doi:10.1093/pastj/gtm047

Lewicki, M., \& Wigger, B. U. (2013). Wer Ist Von Altersarmut Bedroht? [Who Is in Threatened by Old Age Poverty?]. Wirtschaftsdienst, 93(7), 462-465.

Lietzmann, P. (2017, 10.03.2017). Das Rentensystem Ist Ungerecht: "Ich Muss Zahlen Und Nur Die Aktuellen Alten Profitieren!" [the Pension Scheme Is Unfair: "I Have to Pay and Only Current Pensioners Profit!"]. Focus Online.

Lindley, S. E., Harper, R., \& Sellen, A. (2008). Designing for Elders: Exploring the Complexity of Relationships in Later Life. Paper presented at the Proceedings of the 22nd British HCI Group Annual Conference on People and Computers: Culture, Creativity, Interaction-Volume 1.

Lloyd, L. (2015). The Fourth Age. In J. Twigg \& W. Martin (Eds.), Routledge Handbook of Cultural Gerontology (pp. 261-268). London \& New York: Routledge.

Lorber, J. (1993). Believing Is Seeing: Biology as Ideology. Gender \& Society, 7(4), 568581.

Lorber, J. (1996). Beyond the Binaries: Depolarizing the Categories of Sex, Sexuality, and Gender. Sociological Inquiry, 66(2), 143-160. doi:10.1111/j.1475682X.1996.tb00214.X

Lorey, I., Ludwig, G., \& Sonderegger, R. (2016). Foucaults Gegenwart. Sexualität - Sorge - Revolution [Foucaults Present. Sexuality - Care - Revolution]. Wien, Linz, Berlin, London, Zürich \& Malaga: transversal texts.

Lyons, I. (2009). Public Perception of Older People and Ageing: A Literature Review. Retrieved from Dublin:

http://www.ncpop.ie/userfiles/file/ncpop\%20reports/Review\%201\%20LR\%20Olde r\%20people\%20and\%20ageing.pdf 
Mac an Ghaill, M. (2003). Men and Masculinities: Theory, Research and Social Practice. London: Open University Press.

Machin, D., \& Van Leeuwen, T. (2007). Global Media Discourse: A Critical Introduction. Abingdon: Routledge.

Madden, J. (2012). The Best Exotic Marigold Hotel [Feature Film]. UK: 20th Century Fox. Maier, C. S. (1977). The Politics of Productivity: Foundations of American International Economic Policy after World War Ii. International Organization, 31(4), 607-633. Mandelbaum, J. (2013, 30.07.2013). Alive and Kicking: The Changing View of Older People on the Silver Screen. The Guardian Weekly.

Mann, R., Tarrant, A., \& Leeson, G. W. (2016). Grandfatherhood: Shifting Masculinities in Later Life. Sociology, 50(3), 594-610. doi:doi:10.1177/0038038515572586

Marshall, L., \& Lipscomb, V. B. (2010). Staging Age: The Performance of Age in Theatre, Dance, and Film. London: Palgrave Macmillan.

Martschukat, J., \& Stieglitz, O. (2018). Geschichte Der Männlichkeiten - Eine Historische Einführung [the History of Masculinity - a Historical Introduction] (Vol. 5).

Frankfurt a. Main: Campus

Mason, J. (2017). Qualitative Researching. Newbury Park: Sage.

Matzler, K., \& Renzl, B. (2005). Patterns in Management Research: An Analysis of Us American, European and German Approaches. Problems and Perspectives in Management, 1(1), 118-127.

Max-Planck-Gesellschaft. (2014). How Do Germans Tick?Retrieved from https://www.mpikg.mpg.de/5483753/How-do-Germans-tick---Dez-14.pdf Maxwell, J. A. (2012). A Realist Approach for Qualitative Research. Los Angeles, London, New Delhi, Singapore \& Washington D.C.: Sage.

McAdams, D. P. (2001). The Psychology of Life Stories. Review of General Psychology, $5(2), 100-122$.

McAdams, D. P. (2015). Life Story. In S. K. Whitbourne (Ed.), The Encyclopedia of Adulthood and Aging. New York: John Wiley \& Sons.

McAdams, D. P., \& McLean, K. C. (2013). Narrative Identity. Current Directions in Psychological Science, 22(3), 233-238. doi:10.1177/0963721413475622

McCormick, R. W. (2001). Gender and Sexuality in Weimar Modernity. Berlin: Springer. McDonald, M., Gough, B., Wearing, S., \& Deville, A. (2017). Social Psychology, Consumer Culture and Neoliberal Political Economy. Journal for the Theory of Social Behaviour, 47(3), 363-379.

Merriam, S. B. (2007). Qualitative Research and Case Study Applications in Education. San Francisco: Jossey-Bass. 
Merriam, S. B. (2009). Qualitative Research: A Guide to Design and Implementation. San Francisco: Jossey-Bass.

Meuser, M. (2016). Modernized Masculinities? Continuities, Challenges and Changes in Men's Lives. In S. E. T. Johansson (Ed.), Among Men: Moulding Masculinities [EBook] (Vol. 1, pp. Chapter 8). Abingdon: Routledge.

Meyers, N. (2003). Something's Gotta Give [Feature Film]. U.S.: Columbia Pictures \& Warner Brothers.

Miles, M. B., \& Huberman, A. M. (1994). Qualitative Data Analysis: An Expanded Sourcebook. Newbury Park: Sage.

Milestone, K., \& Meyer, A. (2012). Gender and Popular Culture. Cambridge: Polity. Miller, T., Govil, N., McMurria, J., Maxwell, R., \& Wang, T. (2005). Global Hollywood 2. London: Palgrave Macmillan.

Milner, C., Van Norman, K., \& Milner, J. (2012). The Media's Portrayal of Ageing.Retrieved from http://www.hsph.harvard.edu/pgda/working.htm

Moeller, R. G. (1998). The" Remasculinization" of Germany in the 1950s: Introduction. Signs: Journal of Women in Culture and Society, 24(1), 101-106.

Moglen, H. (2008a). Ageing and Transageing: Transgenerational Hauntings of the Self. Studies in Gender and Sexuality, 9(4), 297-311. doi:10.1080/15240650802370668

Moglen, H. (2008b). Feminism, Transageing, and Ageism: A Response to Lynne Segal. Studies in Gender and Sexuality, 9(4), 323-327. doi:10.1080/15240650802370759

Mojo Box Office. (2010). The Expendables. Retrieved from http://www.boxofficemojo.com/movies/?id=expendables.htm

Mojo Box Office. (2012). The Expendable 2. Retrieved from http://www.boxofficemojo.com/movies/?id=expendables $2 . h t m$

Mojo Box Office. (2014). The Expendables 3. Retrieved from

http://www.boxofficemojo.com/movies/?id=expendables3.htm

Moore, A., \& Reynolds, P. (2016). Against the Ugliness of Age: Towards an Erotics of the Aging Sexual Body. Interalia: A Journal of Queer Studies, 11a(2016), 88-105.

Morley, C. (2008). Critical Reflection as a Research Methodology. In P. Liamputtong \& J. Rumbold (Eds.), Knowing Differently: Arts-Based and Collaborative Research Methods (pp. 265-280). Hauppauge: Nova.

Mosse, G. L. (1998). The Image of Man: The Creation of Modern Masculinity. Oxford: Oxford University Press.

Mulvey, L. (1992 [1975]). "Visual Pleasure and Narrative Cinema, "the Sexual Subject: A Screen Reader in Sexuality. London \& New York: Routledge. 
Murray, M. (2003). Narrative Psychology. In J. A. Smith (Ed.), Qualitative Psychology: A Practical Guide to Research Methods (pp. 111-131). Newbury Park: Sage.

Murtaugh, T. (2017, 02.02.2017). 62 Celebrities Who Have Only Gotten Better-Looking with Age. Helen Mirren — Once a Dame, Always a Dame. Women's Day.

Myers, T. (2004). Slavoj Zizek. Abingdon: Routledge.

Neale, S. (1983). Masculinity as Spectacle: Reflections on Men and Mainstream Cinema. Screen, 24, 2 - 17.

Needleman, J. (1994). Money and the Meaning of Life. New York: Crown Business.

Negra, D., \& Tasker, Y. (2014). Gendering the Recession: Media and Culture in an Age of Austerity. Durham: Duke University Press.

Negra, D., Tasker, Y., \& McRobbie, A. (2007). Interrogating Postfeminism: Gender and the Politics of Popular Culture. Durham, U.S.: Duke University Press.

Nelson, H. L., \& Lindemann, H. (2001). Damaged Identities, Narrative Repair. Ithaca: Cornell University Press.

Nelson, T. D. (2005). Ageism: Prejudice against Our Feared Future Self. Journal of Social Issues, 61(2), 207-221.

Nilsson, M., Hagberg, J.-E., \& Grassman, E. J. (2013). To Age as a Man: Ageing and Masculinity in a Small Rural Community in Sweden. norma, 8(1), 59-76.

OECD. (2015). Pensions at a Glance 2015: OECD Publishing.

Orr, C. (2010, 15.10.2010). 'Red':Willis, Freeman, and Co.Channel Van Damme...And It's Bad. The Atlantic.

Palmore, E. (2001). The Ageism Survey: First Findings. The Gerontologist, 41(5), 572575.

Patterson, C. (2014, 29.10.2014). Helen Mirren Shows Women Can Age Beautifully, but We Shouldn't Have To. The Guardian.

Patton, M. Q. (2014). Qualitative Research and Evaluation Methods (4. edition ed.). Thousand Oaks: Sage

Phoenix, C., \& Griffin, M. (2015). Sport, Physical Activity and Ageing. In J. Twigg \& W. Martin (Eds.), The Routledge Handbook of Cultural Gerontology (pp. 329-336). London \& New York: Routledge.

Phoenix, C., \& Sparkes, A. C. (2006). Young Athletic Bodies and Narrative Maps of Aging. Journal of Aging Studies, 20(2), 107-121.

Pickard, S. (2019). Age War as the New Class War? Contemporary Representations of Intergenerational Inequity. Journal of Social Policy, 48(2), 369-386.

Poiger, U. G. (1998). A New, 'Western' Hero? Reconstructing German Masculinity in the 1950s. Signs: Journal of Women in Culture \& Society, 24(1), 147. 
Price, D., \& Livsey, L. (2015). Money and Later Life. In J. Twigg \& W. Martin (Eds.), Routledge Handbook of Cultural Gerontology (pp. 305-312). London \& New York: Routledge.

Pyke, K. D. (1996). Class-Based Masculinities. Gender \& Society, 10(5), 527-549. doi:doi:10.1177/089124396010005003

Reeser, T. W. (2011). Masculinities in Theory: An Introduction. Hoboken: John Wiley \& Sons.

Regmi, K., Naidoo, J., \& Pilkington, P. (2010). Understanding the Processes of Translation and Transliteration in Qualitative Research. International Journal of Qualitative Methods, 9(1), 16-26.

Richardson, N. (2016). Transgressive Bodies: Representations in Film and Popular Culture. London \& New York: Routledge.

Richardson, N. (2019). Ageing Femininity on Film: The Older Woman in Contemporary Cinema. London, New York, Oxford, New Delhi \& Sydney: IB Tauris.

Richardson, N., \& Wearing, S. (2014). Gender in the Media. New York: Palgrave Macmillan.

Ritchie, J., Lewis, J., Nicholls, C. M., \& Ormston, R. (2013). Qualitative Research Practice: A Guide for Social Science Students and Researchers. Newbury Park: Sage.

Roberson, J. E., \& Suzuki, N. (2005). Men and Masculinities in Contemporary Japan: Dislocating the Salaryman Doxa (Vol. 10). London \& New York: Routledge. Robertson, L., \& Hale, B. (2011). Interviewing Older People; Relationships in Qualitative Research. Internet Journal of Allied Health Sciences and Practice, 9(3), 1-8.

Rose, G. (2016). Visual Methodologies: An Introduction to Researching with Visual Materials. Newbury Park: Sage.

Rowe, J. W., \& Kahn, R. L. (1997). The Structure of Successful Aging. Gerontologist, $37(4), 433-440$.

Rowe, J. W., \& Kahn, R. L. (1998). Successful Aging: The Macarthur Foundation Study. New York: Pantheon.

Rubin, G. (1997). Thinking Sex: Notes for a Radical Theory of the Politics of Sexuality. In P. M. N. B. E. Schneider (Ed.), Social Perspectives in Lesbian and Gay Studies; a Reader (pp. 100-133). London \& New York: Routledge.

Russell, C. (1999). Interviewing Vulnerable Old People: Ethical and Methodological Implications of Imagining Our Subjects. Journal of Aging Studies, 13(4), 403-417. doi:http://dx.doi.org/10.1016/S0890-4065(99)00018-3 
Sandberg, L. (2011). Getting Intimate: A Feminist Analysis of Old Age, Masculinity and Sexuality. (PhD), Linköping University, Linköping University Electronic Press.

Retrieved from http://liu.divaportal.org/smash/get/diva2:408208/FULLTEXT01.pdf

Sandberg, L. (2013). Affirmative Old Age - the Ageing Body and Feminist Theories on Difference. International Journal of Ageing and Later Life, 8(1), 11-40.

Saxton, B., \& Cole, T. R. (2013). No Country for Old Men: A Search for Masculinity in Later Life. International Journal of Ageing and Later Life, 7(2), 97-116.

Schatz, T., \& Perren, A. (2004). Hollywood. In J. D. H. Downing, D. McQuail, P. Schlesinger, \& E. A. Wartella (Eds.), The Sage Handbook of Media Studies (pp. 495-515). London: Sage.

Scheibe, S., Kunzmann, U., \& Baltes, P. B. (2007). Wisdom, Life Longings, and Optimal Development. In J. A. B. C. N. Dulmus (Ed.), Handbook of Gerontology: Evidence-Based Approaches to Theory, Practice, and Policy (pp. 117-142). Hoboken: Wiley.

Schippers, M. (2007). Recovering the Feminine Other: Masculinity, Femininity, and Gender Hegemony. Theory and Society, 36(1), 85-102.

Schommer-Aikins, M. (2004). Explaining the Epistemological Belief System: Introducing the Embedded Systemic Model and Coordinated Research Approach. Journal of Educational Psychologist, 39(1), 19-29.

Schommer-Aikins, M., \& Easter, M. (2006). Ways of Knowing and Epistemological Beliefs: Combined Effect on Academic Performance. Educational Psychology, 26(3), 411-423. doi:10.1080/01443410500341304

Schommer, M. (1990). Effects of Beliefs About the Nature of Knowledge on Comprehension. Journal of Educational Psychology, 82(3), 498-504.

Schön, D. A. (1983). The Reflective Practitioner: How Professionals Think in Action. New York: Basic Books.

Schütz, A. (1967). The Phenomenology of the Social World. Evanston: Northwestern University Press.

Schütz, A. (1970). Some Structures of the Life-World. In I. Schutz (Ed.), Collected Papers Iii; Studies in Phenomenological Philosophy (pp. 116-132). Berlin: Springer.

Schweinitz, J. (2011). Film and Stereotype: A Challenge for Cinema and Theory. New York: Columbia University Press.

Schwenkte, R. (2010). R.E.D. - Retired and Extremely Dangerous [Feature Film]. U.S.: Summit Entertainment. 
Scotland, J. (2012). Exploring the Philosophical Underpinnings of Research: Relating Ontology and Epistemology to the Methodology and Methods of the Scientific, Interpretive, and Critical Research Paradigms. English Language Teaching, 5(9), 916.

Segal, L. (2008). All Ages and None: Commentary on Helene Moglen's Ageing and Transageing. Studies in Gender and Sexuality, 9(4), 312-322. doi:10.1080/15240650802370734

Segal, L. (2013). Out of Time: The Pleasures and the Perils of Ageing. London \& New York: Verso Books.

Segal, L. (2014). Temporal Vertigo: The Paradoxes of Ageing. Studies in Gender and Sexuality, 15(3), 214-222. doi:10.1080/15240657.2014.939022

Shary, T. (2013). Millennial Masculinity: Men in Contemporary American Cinema. Detroit: Wayne State University Press.

Silverman, D. (2016). Qualitative Research. Newbury Park: Sage.

Singer, M. (2014, 04.03.2014). Op-Ed: The Slow and Slightly Creaky Rise of the Old-Man Action Movie. Newsreel.

Sloan, C., Gough, B., \& Conner, M. (2010). Healthy Masculinities? How Ostensibly Healthy Men Talk About Lifestyle, Health and Gender. Psychology \& Health, 25(7), 783-803. doi:10.1080/08870440902883204

Smith, J. A. (2015). Qualitative Psychology: A Practical Guide to Research Methods. Newbury Park: Sage.

Smith, K. (2010, 15.10.2010). Granny Get Your Gun! New York Post.

Sontag, S. (1972). The Double Standard of Aging. The Saturday Review

Spector-Mersel, G. (2006). Never-Aging Stories: Western Hegemonic Masculinity Scripts. Journal of Gender Studies, 15(1), 67-82.

Stallone, S. (2010). The Expendables (Director's Cut) [Feature Film]. U.S.: Lionsgate.

Steiner, J. M. (2013). Power Politics and Social Change in National Socialist Germany: A Process of Escalation into Mass Destruction (Vol. 11). Berlin: Walter de Gruyter.

Stewart, J., \& Sambrook, S. (2008). Developing Critical Reflection in Professional Focused Doctorates: A Facilitator's Perspective. Journal of European Industrial Training, 32(5), 359-373. doi:doi:10.1108/03090590810877085

Strydom, P. (2011). Contemporary Critical Theory and Methodology. Oxon \& New York: Routledge.

Swinnen, A., \& Stotesbury, J. A. (2012). Aging, Performance, and Stardom: Doing Age on the Stage of Consumerist Culture (Vol. 2). Münster: LIT Verlag Münster. 
Tarrant, A. (2010). 'Maturing' a Sub-Discipline: The Intersectional Geographies of Masculinities and Old Age. Geography Compass, 4(10), 1580-1591. doi:10.1111/j.1749-8198.2010.00394.x

Tasker, Y. (1993a). Dumb Movies for Dumb People: Masculinity, the Body, and the Voice in Contemporary Action Cinema. In S. Cohan \& I. R. Hark (Eds.), Screening the Male: Exploring Masculinities in Hollywood Cinema (pp. 230-244). London \& New York: Routledge.

Tasker, Y. (1993b). Spectacular Bodies: Gender, Genre and the Action Cinema. New York: Routledge.

Tasker, Y. (2004). Action and Adventure Cinema. London: Routledge.

Tasker, Y. (2012). Spectacular Bodies: Gender, Genre and the Action Cinema. New York: Routledge.

Tasker, Y. (2015). The Hollywood Action and Adventure Film. London: Routledge.

Tasker, Y., \& Negra, D. (2007). Interrogating Postfeminism: Gender and the Politics of Popular Culture. In Y. Tasker \& D. Negra (Eds.), (pp. 1-26). Durham: Duke University Press.

Taylor, F. (2011). Exorcising Hitler: The Occupation and Denazification of Germany. Bloomsbury: Bloomsbury Publishing.

Temple, B., \& Young, A. (2004). Qualitative Research and Translation Dilemmas. Qualitative Research, 4(2), 161-178.

Thompson, E. H. (1994). Older Men's Lives (Vol. 6). Newbury Park: Sage

Thompson, E. H. (2006). Images of Old Men's Masculinity: Still a Man? Sex Roles, 55(910), 633-648. doi:10.1007/s11199-006-9119-7

Thompson, E. H., \& Whearty, P. M. (2004). Older Men's Social Participation: The Importance of Masculinity Ideology. Journal of Men's Studies, 13(1), 5-24.

Thompson, P. R., Itzin, C., \& Abendstern, M. (1990). I Don't Feel Old: The Experience of Later Life. Oxford \& New York: Oxford University Press.

Thornton, J. E. (2002). Myths of Aging or Ageist Stereotypes. Educational Gerontology, 28(4), 301-312.

Tjeder, D. (2003). The Power of Character: Middle-Class Masculinities, 1800-1900. Stockholm: Stockholm University.

Trachtman, R. (1999). The Money Taboo: Its Effects in Everyday Life and in the Practice of Psychotherapy. Clinical Social Work Journal, 27(3), 275-288. doi:10.1023/a:1022842303387

Treme, J., \& Craig, L. A. (2013). Celebrity Star Power: Do Age and Gender Effects Influence Box Office Performance? Applied Economics Letters, 20(5), 440-445. 
Tucker, R. (2014, 2014-02-16). Hollywood Loves Its Aging Action Heroes. New York PostRetrieved from http://nypost.com/2014/02/16/ready-aim-retired/

Tulle, E. (2015). Theorising Embodiment and Ageing. In Julia Twigg \& W. Martin (Eds.), The Routledge Handbook of Cultural Gerontology (pp. 125-133). London \& New York: Routledge.

Turner III, D. W. (2010). Qualitative Interview Design: A Practical Guide for Novice Investigators. The Qualitative Report, 15(3), 754-760.

Twigg, J., \& Martin, W. (2015). Routledge Handbook of Cultural Gerontology. London \& New York: Routledge.

University of Gloucestershire. (2008). Research Ethics: A Handbook of Principles and Procedures.Retrieved from Gloucestershire:

https://www.glos.ac.uk/docs/download/Research/handbook-of-principles-andprocedures.pdfhttps://www.glos.ac.uk/docs/download/Research/handbook-ofprinciples-and-procedures.pdf

van Dyk, S. (2014). The Appraisal of Difference: Critical Gerontology and the ActiveAgeing-Paradigm. Journal of Aging Studies, 31, 93-103.

van Nes, F., Abma, T., Jonsson, H., \& Deeg, D. (2010). Language Differences in Qualitative Research: Is Meaning Lost in Translation? European Journal of Ageing, 7(4), 313-316. doi:10.1007/s10433-010-0168-y

van Rahden, T. (2010). Wie Vati Die Demokratie Lernte: Religion, Familie Und Die Frage Der Autorität in Der Frühen Bundesrepublik [How Daddy Learned About Democracy: Religion, Family and the Question of Authority in the Early Federal Republic of Germany]. In D. Fulda, D. Herzog, S. L. Hoffmann, \& T. v. Rahden (Eds.), Demokratie Im Schatten Der Gewalt: Geschichten Des Privaten Im Deutschen Nachkrieg [Democracy in the Shaddow of Violence: Stories of the Private in Germany after the War] (pp. 122-151). Göttingen: Wallstein. Victor, C. R. (1994). Old Age in Modern Society (2. ed.). Berlin: Springer. Wals, A. E. J., \& Jickling, B. (2002). "Sustainability" in Higher Education: From Doublethink and Newspeak to Critical Thinking and Meaningful Learning. International Journal of Sustainability in Higher Education, 3(3), 221-232.

Weber, M. (2013). The Protestant Ethic and the Spirit of Capitalism. Abington: Routledge. Weedon, C. (2004). Identity and Culture: Narratives of Difference and Belonging. New York: McGraw-Hill Education.

Wells, B. E., \& Twenge, J. M. (2005). Changes in Young People's Sexual Behavior and Attitudes, 1943-1999: A Cross-Temporal Meta-Analysis. Review of General Psychology, 9(3), 249-261. 
West, S. (Writer). (2012). The Expendables 2 (Director's Cut) [Feature Film]. U.S.:

Lionsgate.

WHO. (2011a). Definition of an Older or Elderly Person. Retrieved from

http://www.who.int/healthinfo/survey/ageingdefnolder/en/

WHO. (2011b). Global Health and Aging.Retrieved from Geneva:

Wierzbicka, A. (2015). Can There Be Common Knowledge without a Common Language?

German Pflicht Versus English Duty. Common Knowledge, 21(1), 141-171.

Willems, E. (1986). A Way of Life and Death!: Three Centuries of Prussian-German

Militarism - an Anthropological Approach. Nashville: Vanderbilt University Press.

Wilson, G. (2000). Understanding Old Age: Critical and Global Perspectives. London,

Thousand Oaks \& New Delhi: Sage.

Witt, M. A., \& Redding, G. (2009). Culture, Meaning, and Institutions: Executive

Rationale in Germany and Japan. Journal of International Business Studies, 40(5), 859-885.

Wittig, M. (1980). The Straight Mind. Feminist Issues, 1(1), 103-111.

Wittig, M. (1993). One Is Not Born a Woman. In H. Abelove, M. A. Barale, \& D. M. Halperin (Eds.), The Lesbian and Gay Studies Reader (pp. 103-109). Abingdon \& New York: Routledge.

Wodak, R., \& Meyer, M. (2009). Methods for Critical Discourse Analysis (2. ed.). Los Angeles, London, New Delhi, Singapore, Washington: Sage.

Wood, J. T. (1994). Gendered Lives: Communication, Gender and Culture. Boston:

Wadsworth Publishing.

Woodrow, L. (2006). Anxiety and Speaking English as a Second Language. RELC Journal, 37(3), 308-328.

Woodward, K. M. (1988). Youthfulness as a Masquerade. Discourse, 11(1), 119-142.

Woodward, K. M. (2003). Against Wisdom: The Social Politics of Anger and Aging. Journal of Aging Studies, 17(1), 55-67.

Woodward, K. M. (2006). Performing Age, Performing Gender. NWSA Journal, 18(1), 162-189.

Wörsching, M. (1999). Metaphors of Hegemonic Masculinity--an Analysis of Sport and Advertising in the German Newsmagazine Der Spiegel. Journal of European Area Studies, 7(2), 177.

Yin, R. K. (2013). Case Study Research: Design and Methods (5. ed.). Los Angeles, London, Singapore, New Delhi \& Washington DC: Sage. 
Zimmer, A., \& Toepler, S. (1996). Cultural Policies and the Welfare State: The Cases of Sweden, Germany, and the United States. The Journal of Arts Management, Law, and Society, 26(3), 167-193. doi:10.1080/10632921.1996.9942961

Žižek, S. (1989). The Undergrowth of Enjoyment: How Popular Culture Can Serve as an Introduction to Lacan. New Formations, 9(Winter 1989), 7-29.

Žižek, S. (1992). Looking Awry: An Introduction to Jacques Lacan through Popular Culture. Cambridge: MIT press.

Žižek, S. (1997). The Plague of Fantasies. London \& New York: Verso.

Žižek, S. (2006). How to Read Lacan. London: Granta.

Žižek, S. (2008). The Sublime Object of Ideology. London \& New York: Verso.

Žižek, S. (2009). First as Tragedy, Then as Farce. London \& New York: Verso.

Žižek, S. (Writer) \& S. Zizek (Director). (2010). Faktor X. Das Ding Und Die Leere

[Factor X. The Thing and the Void] [Audio Book]. In Supposé (Producer):

Supposé. 


\section{Appendix}

A Informed Consent

\section{Informed Consent}

Research Project: Doctoral thesis with the aim to collect the experiences of older German men with ageing and older age and to analyse with reference to the sociocultural context.

Durchführende Institution: University of Gloucestershire

Interviewerin: Lisa-Nike Bühring

Interview Date:

Description oft he Research Project:

oral explanation

$\square \quad$ written explanation

I, consent to giving one or several interviews for the above described research project if the following conditions are fulfilled. I am aware of the fact that participation in one or more interviews is voluntary and that I can withdraw my consent at any point and without justification and negative consequences. I can also object to the storage of my data at any point and demand their deletion.

I declare my consent with the following:

$1 \quad$ Recording

Recording of the interview on one or more sound recording devices

2 Transcription of the recording in:

$\square \quad$ anonymised form (no name mentioned)

$\square \quad$ non-anonymised form

3 Publication

$\square \quad$ completely

$\square \quad$ extracts as quotes 
In return, I expect of the researcher the following:

$\square \quad$ a copy of the interview conducted with me before publication for review $\square \quad$ debriefing after the interview

After the completion of the research project, the recording should be

$\square \quad$ erased by the researcher

$\square \quad$ stored by the researcher

Date:

Signature (Interviewee):

Interviewer:

Lisa Bühring, M.A.
Supervisor:

Prof. Dr Ros Jennings

PH008 Principals House

Francis Close Hall

Swindon Road

GL50 4AZ, Cheltenham, UK 


\section{Einverständniserklärung}

Forschungsprojekt: Promotion mit dem Ziel die Erfahrungen älterer deutscher Männer mit dem Altern und älter werden zu sammeln und unter Einbeziehung des soziokulturellen Kontextes zu analysieren.

Durchführende Institution: University of Gloucestershire

Interviewerin: Lisa-Nike Bühring, M.A.

Interviewdatum: 17.072017

Beschreibung des Forschungsprojekts:

\& mündliche Erläuterungen

schriftliche Erläuterungen

Ich, bin, unter unten folgenden Bedingungen, damit einverstanden im besprochenen Forschungsprojekt ein oder mehrere Interviews zu geben. Mir ist bewusst, dass die Teilnahme an einem oder mehreren Interviews freiwillig ist und ich mein Einverständnis dazu jederzeit ohne Begründung und ohne Nachteile zurückziehen kann. Ebenso kann ich einer Speicherung meiner jederzeit widersprechen und deren Löschung verlangen.

Ich erkläre mich mit folgenden Punkten einverstanden:

\section{Aufnahme}

\ Aufzeichnung des Interviews auf einen oder mehrere Tonträger

2 Verschriftlichung der Tonaufzeichnungen in:

$\triangle \quad$ anonymisierter Form (keine Namensnennung)

カ nicht anonymisierter Form

3 Veröffentlichung

《 zu wissenschaftlichen Zwecken

$\square \quad$ vollständig

凶. ausschnittsweise als Zitat 
$\square \quad$ Veröffentlichung erst nach Rücksprache

Im Gegenzug erwarte ich von der Interviewerin folgende Leistungen:

\& ein Exemplar des Transkripts des mit mir geführten Interviews vor der Veröffentlichung zur Prüfung

$\square \quad$ eine Nachbesprechung des Interviews

$\square \quad$ einen weiteren Termin für Fragen bzw. um einen Einblick in die Ergebnisse zu erhalten

\section{Nach Beendigung des Projekts soll die Tonaufnahme}

¿ von der Forscherin gelöscht werden

$\square \quad$ von der Forscherin verwahrt werden

Datum: 17.07 .2017

Unterschrift (Befragter):

Interviewerin:

Lisa Bühring, M.A.
Doktormutter:

Prof. Dr. Ros Jennings

PH008 Principals House

Francis Close Hall

Swindon Road

GL50 4AZ, Cheltenham, UK 
UNIVERSITY OF

GLOUCESTERSHIRE

at Cheltenham and Cloucester

\section{Einverständniserklärung}

Forschungsprojekt: Promotion mit dem Ziel die Erfahrungen älterer deutscher Männer mit dem Altern und älter werden zu sammeln und unter Einbeziehung des soziokulturellen Kontextes zu analysieren.

Durchführende Institution: University of Gloucestershire

Interviewerin: Lisa-Nike Bühring, M.A.

Interviewdatum: 18.072017

Beschreibung des Forschungsprojekts:

Q mündliche Erläuterungen

$\square \quad$ schriftliche Erläuterungen

Ich, bin, unter unten folgenden Bedingungen, damit einverstanden im besprochenen Forschungsprojekt ein oder mehrere Interviews zu geben. Mir ist bewusst, dass die Teilnahme an einem oder mehreren Interviews freiwillig ist und ich mein Einverständnis dazu jederzeit ohne Begründung und ohne Nachteile zurückziehen kann. Ebenso kann ich einer Speicherung meiner jederzeit widersprechen und deren Löschung verlangen.

Ich erkläre mich mit folgenden Punkten einverstanden:

\section{Aufnahme}

\ Aufzeichnung des Interviews auf einen oder mehrere Tonträger

2 Verschriftlichung der Tonaufzeichnungen in:

$\square \quad$ anonymisierter Form (keine Namensnennung)

$\not$ nicht anonymisierter Form

3 Veröffentlichung zu wissenschaftlichen Zwecken

$\bowtie \quad$ vollständig

$\square \quad$ ausschnittsweise als Zitat 
UNIVERSITY OF

GLOUCESTERSHIRE

at Cheltenham and Clouceste

Im Gegenzug erwarte ich von der Interviewerin folgende Leistungen:

$\notin$ ein Exemplar des Transkripts des mit mir geführten Interviews vor der Veröffentlichung zur Prüfung

$\square \quad$ eine Nachbesprechung des Interviews

Nach Beendigung des Projekts soll die Tonaufnahme

$\square \quad$ von der Forscherin gelöscht werden

$\notin$ von der Forscherin verwahrt werden

Datum: 18.07 .2017

Unterschrift (Befragter):

Interviewerin:

Lisa Bühring, M.A.
Doktormutter:

Prof. Dr. Ros Jennings

PH008 Principals House

Francis Close Hall

Swindon Road

GL50 4AZ, Cheltenham, UK 
UNIVERSITY OF

GLOUCESTERSHIRE

at Cheltenham and Cloucester

\section{Einverständniserklärung}

Forschungsprojekt: Promotion mit dem Ziel die Erfahrungen älterer deutscher Männer mit dem Altern und älter werden zu sammeln und unter Einbeziehung des soziokulturellen Kontextes zu analysieren.

Durchführende Institution: University of Gloucestershire

Interviewerin: Lisa-Nike Bühring, M.A.

Interviewdatum: 15.12 .2017

Beschreibung des Forschungsprojekts:

$\mathrm{X} \square$ mündliche Erläuterungen

$\square \quad$ schriftliche Erläuterungen

Ich, Gerd Becker bin, unter unten folgenden Bedingungen, damit einverstanden im besprochenen Forschungsprojekt ein oder mehrere Interviews zu geben. Mir ist bewusst, dass die Teilnahme an einem oder mehreren Interviews freiwillig ist und ich mein Einverständnis dazu jederzeit ohne Begründung und ohne Nachteile zurückziehen kann. Ebenso kann ich einer Speicherung meiner jederzeit widersprechen und deren Löschung verlangen.

Ich erkläre mich mit folgenden Punkten einverstanden:

1 Aufnahme

$\square \mathrm{X}$ Aufzeichnung des Interviews auf einen oder mehrere Tonträger

2 Verschriftlichung der Tonaufzeichnungen in:

$\square \mathrm{X}$ anonymisierter Form (keine Namensnennung)

$\square \quad$ nicht anonymisierter Form

3 Veröffentlichung zu wissenschaftlichen Zwecken

$\square \quad$ vollständig

$\square \mathrm{X}$ ausschnittsweise als Zitat 


\section{UNIVERSITY OF}

GLOUCESTERSHIRE

at Cheltenham and Gloucester

Im Gegenzug erwarte ich von der Interviewerin folgende Leistungen:

$\square X$ ein Exemplar des Transkripts des mit mir geführten Interviews vor der Veröffentlichung zur Prüfung

$\square \mathrm{X} \quad$ eine Nachbesprechung des Interviews

Nach Beendigung des Projekts soll die Tonaufnahme

$\square \mathbf{X}$ von der Forscherin gelöscht werden

$\square \quad$ von der Forscherin verwahrt werden

Datum: 15.12.2017

Unterschrift (Befragter):

Gerd Becker

Interviewerin:

Lisa Bührina. M.A.

Doktormutter:

Prof. Dr. Ros Jennings

PH008 Principals House

Francis Close Hall

Swindon Road

GL50 4AZ, Cheltenham, UK 
UNIVERSITY OF

GLOUCESTERSHIRE

at Cheltenham and Cloucester

\section{Einverständniserklärung}

Forschungsprojekt: Promotion mit dem Ziel die Erfahrungen älterer deutscher Männer mit dem Altern und älter werden zu sammeln und unter Einbeziehung des soziokulturellen Kontextes zu analysieren.

Durchführende Institution: University of Gloucestershire

Interviewerin: Lisa-Nike Bühring, M.A.

Interviewdatum: 31072018

Beschreibung des Forschungsprojekts:

mündliche Erläuterungen

$\square \quad$ schriftliche Erläuterungen

Ich, bin, unter unten folgenden

Bedingưrgen, damit einverstanden im besprochenen Forschungsprojekt ein oder mehrere Interviews zu geben. Mir ist bewusst, dass die Teilnahme an einem oder mehreren Interviews freiwillig ist und ich mein Einverständnis dazu jederzeit ohne Begründung und ohne Nachteile zurückziehen kann. Ebenso kann ich einer Speicherung meiner jederzeit widersprechen und deren Löschung verlangen.

Ich erkläre mich mit folgenden Punkten einverstanden

\section{Aufnahme}

D. Aufzeichnung des Interviews auf einen oder mehrere Tonträger

2 Verschriftlichung der Tonaufzeichnungen in:

$\square \quad$ anonymisierter Form (keine Namensnennung)

d nicht anonymisierter Form

3 Veröffentlichung zu wissenschaftlichen Zwecken

(6) vollständig

$\square \quad$ ausschnittsweise als Zitat 


\section{UNIVERSITY OF}

\section{GLOUCESTERSHIRE}

at Cheltenham and Clouceste

Im Gegenzug erwarte ich von der Interviewerin folgende Leistungen:

ㅇ ein Exemplar des Transkripts des mit mir geführten Interviews vor der Veröffentlichung zur Prüfung

$\square \quad$ eine Nachbesprechung des Interviews

Nach Beendigung des Projekts soll die Tonaufnahme

$\square \quad$ von der Forscherin gelöscht werden

von der Forscherin verwahrt werden

Datum: $31 \cdot 07 \cdot 2018$

Unterschrift (Befragter):

Interviewerin:

Lisa Bühring, M.A.
Doktormutter:

Prof. Dr. Ros Jennings

PH008 Principals House

Francis Close Hall

Swindon Road

GL50 4AZ Cheltenham. UK 


\section{B Interview Questions}

\begin{tabular}{|c|c|c|c|c|}
\hline \begin{tabular}{|l|} 
Questions Pilot \\
Interview \\
$(21.06 .17)$ \\
\end{tabular} & $\begin{array}{l}\text { Questions } \\
\text { interview \#1 } \\
(17.07 .17) \\
\end{array}$ & $\begin{array}{l}\text { Questions Interview \#2 } \\
(18.07 .17)\end{array}$ & $\begin{array}{l}\text { Questions } \\
\text { Interview \#3 } \\
(15.12 .17) \\
\end{array}$ & $\begin{array}{l}\text { Questions } \\
\text { Interview \#4 } \\
(30.07 .18) \\
\end{array}$ \\
\hline & $\begin{array}{l}\text { 1. What does } \\
\text { masculinity } \\
\text { mean to } \\
\text { you? }\end{array}$ & $1 . \quad \hat{=}$ & $1 . \triangleq$ & $1 . \hat{=}$ \\
\hline & & $\begin{array}{l}\text { To what extent has } \\
\text { your understanding } \\
\text { of masculinity been } \\
\text { influenced by your } \\
\text { upbringing/education } \\
\text {..? }\end{array}$ & 2. $\hat{=}$ & 2. $\triangleq$ \\
\hline \begin{tabular}{|l} 
1. What is your \\
highest \\
qualification \\
?
\end{tabular} & 2. $\triangleq$ & 3. $\hat{=}$ & 3. $\triangleq$ & 3. $\hat{=}$ \\
\hline $\begin{array}{c}\text { 2. What was } \\
\text { your job? }\end{array}$ & $\begin{array}{l}\text { 3. Could you } \\
\text { briefly } \\
\text { describe } \\
\text { your career } \\
\text { progression, } \\
\text { please? }\end{array}$ & 4. $\hat{=}$ & 4. $\hat{=}$ & 4. $\hat{=}$ \\
\hline & $\begin{array}{l}\text { 4. Which job } \\
\text { did you have } \\
\text { before you } \\
\text { retired? }\end{array}$ & & & \\
\hline 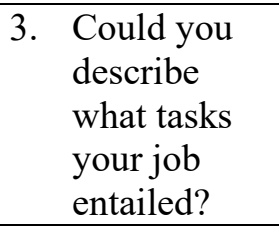 & $\begin{array}{l}\text { 5. Could you } \\
\text { describe } \\
\text { what tasks } \\
\text { your last job } \\
\text { entailed? }\end{array}$ & & & \\
\hline \begin{tabular}{|l|} 
4. Which \\
professional \\
tasks did \\
you enjoy \\
the most? \\
\end{tabular} & $6 . \quad \hat{=}$ & 5. $\hat{=}$ & 5. $\hat{=}$ & 5. $\triangleq$ \\
\hline \begin{tabular}{|ll} 
5. & And which \\
& tasks did \\
& you not like \\
& so much? \\
\end{tabular} & 7. $\hat{=}$ & 6. $\hat{=}$ & 6. $\triangleq$ & 6. $\hat{=}$ \\
\hline \begin{tabular}{|l} 
6. \\
Do you \\
think your \\
job had an \\
influence on \\
how you \\
viewed \\
yourself? If \\
so, how? \\
\end{tabular} & 8. $\triangleq$ & 7. $\hat{=}$ & 7. $\triangleq$ & 7. $\hat{=}$ \\
\hline 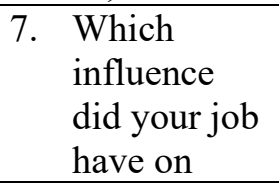 & & & & \\
\hline
\end{tabular}




\begin{tabular}{|c|c|c|c|c|}
\hline $\begin{array}{l}\text { your private } \\
\text { life? }\end{array}$ & & & & \\
\hline \multirow[t]{3}{*}{ 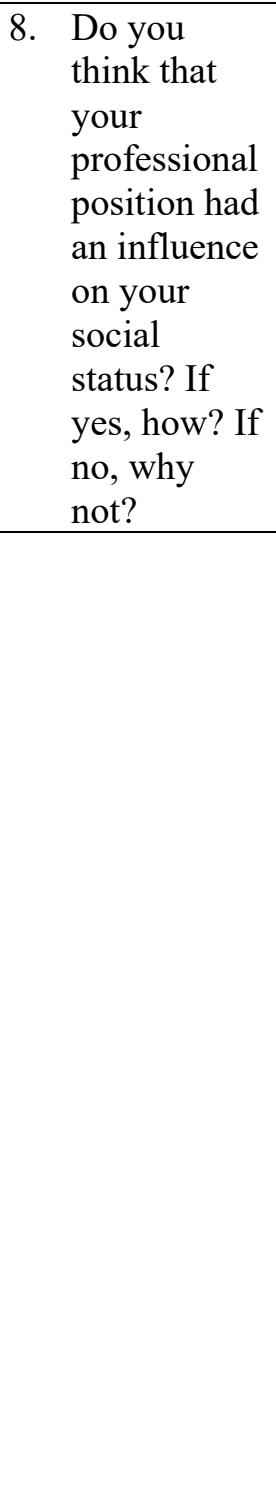 } & $9 . \quad \triangleq$ & 8. $\quad \hat{=}$ & $8 . \quad$ & 8. $\triangleq$ \\
\hline & $\begin{array}{l}\text { 10. If you had } \\
\text { to make a } \\
\text { list of your } \\
\text { priorities } \\
\text { during the } \\
\text { time of } \\
\text { your } \\
\text { employmen } \\
\text { t, what } \\
\text { would be } \\
\text { on top, in } \\
\text { the middle } \\
\text { and at the } \\
\text { end? }\end{array}$ & & & \\
\hline & $\begin{array}{l}\text { 11. How was } \\
\text { your } \\
\text { private life } \\
\text { been } \\
\text { structured } \\
\text { during your } \\
\text { professiona } \\
1 \text { life? } \\
\end{array}$ & 9. $\triangleq$ & 9. $\triangleq$ & 9. $\hat{=}$ \\
\hline $\begin{array}{l}\text { 9. When did } \\
\text { you retire? }\end{array}$ & 12. $\triangleq$ & 10. $\triangleq$ & 10. $\hat{=}$ & 10. $\triangleq$ \\
\hline $\begin{array}{l}\text { 10. How did } \\
\text { you } \\
\text { experience } \\
\text { the } \\
\text { transition } \\
\text { from being } \\
\text { employed to } \\
\text { being } \\
\text { retired? } \\
\end{array}$ & 13. $\hat{=}$ & 11. $\hat{=}$ & 11. $\triangleq$ & 11. $\hat{=}$ \\
\hline \multirow[t]{2}{*}{$\begin{array}{l}\text { 11. How has } \\
\text { your life } \\
\text { changed } \\
\text { since } \\
\text { retirement? }\end{array}$} & $\begin{array}{l}\text { 14. Have your } \\
\text { priorities } \\
\text { changed } \\
\text { since } \\
\text { retirement? } \\
\text { If so, how? } \\
\end{array}$ & 12. $\hat{=}$ & 12. $\triangleq$ & 12. $\hat{=}$ \\
\hline & & $\begin{array}{l}\text { 13. Has your position as } \\
\text { retiree had an } \\
\text { influence on how you } \\
\text { view yourself? If so, } \\
\text { how? }\end{array}$ & $\begin{array}{l}\text { 13. Has } \\
\text { your } \\
\text { posit } \\
\text { ion } \\
\text { as } \\
\end{array}$ & 13. $\triangleq$ \\
\hline
\end{tabular}




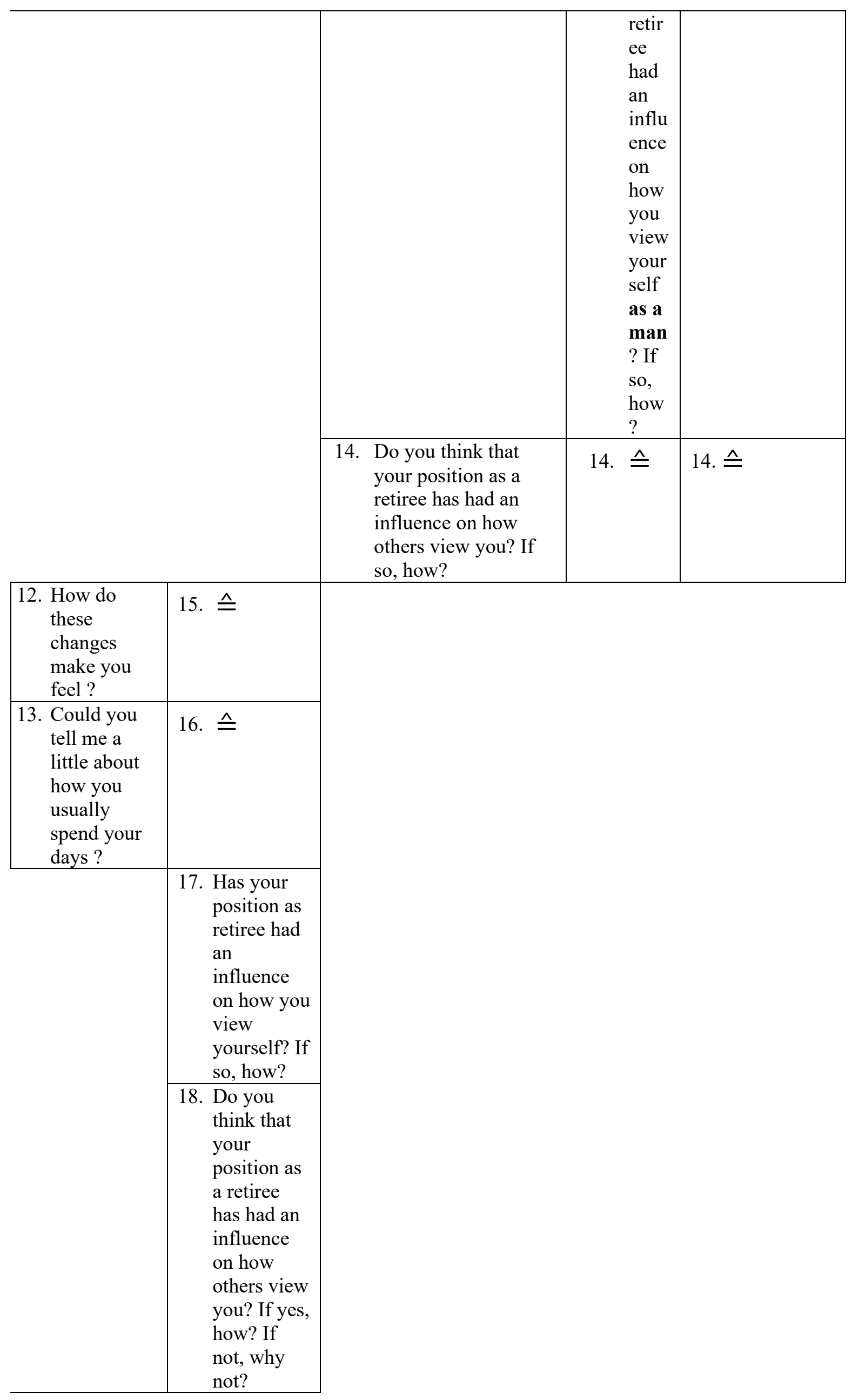




\begin{tabular}{|c|c|c|c|c|}
\hline \begin{tabular}{|l} 
14. Do you live \\
in a \\
relationship?
\end{tabular} & 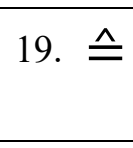 & & & \\
\hline $\begin{array}{l}\text { 15. How old is } \\
\text { your } \\
\text { partner? }\end{array}$ & 20. $\triangleq$ & & & \\
\hline $\begin{array}{l}\text { 16. Is she } \\
\text { retired, too? }\end{array}$ & 21. $\triangleq$ & & & \\
\hline $\begin{array}{l}\text { 17. Has your } \\
\text { relationship } \\
\text { changed } \\
\text { since } \\
\text { retirement? } \\
\text { If yes, how? } \\
\end{array}$ & 22. $\triangleq$ & & & \\
\hline \multicolumn{2}{|l|}{$\begin{array}{l}\text { 18. Do you still } \\
\text { have sexual } \\
\text { intercourse? }\end{array}$} & & & \\
\hline $\begin{array}{l}\text { 19. Who } \\
\text { belongs to } \\
\text { the closer } \\
\text { circle of } \\
\text { your family? }\end{array}$ & 23. $\triangleq$ & & & \\
\hline \multicolumn{5}{|l|}{$\begin{array}{l}\text { 20. How often } \\
\text { do you have } \\
\text { contact with } \\
\text { the closer } \\
\text { circle of } \\
\text { your family? }\end{array}$} \\
\hline $\begin{array}{l}\text { 21. Has your } \\
\text { relationship } \\
\text { to closer } \\
\text { family } \\
\text { members } \\
\text { changed } \\
\text { since } \\
\text { retirement? } \\
\text { If so, how? }\end{array}$ & 24. $\triangleq$ & $\begin{array}{l}\text { 15. Has your relationship } \\
\text { to your wife and } \\
\text { family changed since } \\
\text { retirement? If so, } \\
\text { how? }\end{array}$ & 15. $\hat{=}$ & 15. $\triangleq$ \\
\hline \begin{tabular}{|l|} 
22. Has your \\
circle of \\
friends \\
changed \\
since \\
retirement? \\
If so, how?
\end{tabular} & $25 . \stackrel{ }{=}$ & 16. $\hat{=}$ & 16. $\triangleq$ & 16. $\stackrel{ }{=}$ \\
\hline \multirow[t]{3}{*}{$\begin{array}{l}\text { 23. How old are } \\
\text { you now? }\end{array}$} & 26. $\triangleq$ & 17. $\stackrel{\wedge}{=}$ & 17. $\stackrel{ }{=}$ & 17. $\stackrel{ }{=}$ \\
\hline & & & 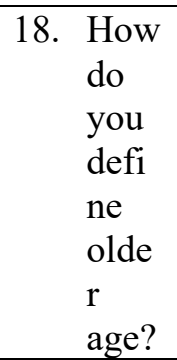 & 18. $\triangleq$ \\
\hline & & & $\begin{array}{l}\text { 19. How } \\
\text { old } \\
\text { do }\end{array}$ & 19. $\triangleq$ \\
\hline
\end{tabular}




\begin{tabular}{|c|c|c|c|c|}
\hline & & & $\begin{array}{l}\text { you } \\
\text { feel } \\
\text { to } \\
\text { be? }\end{array}$ & \\
\hline \begin{tabular}{|l} 
24. Have your \\
mental and \\
physical \\
abilities \\
changed \\
with age? \\
\end{tabular} & $\begin{array}{l}\text { 27. Have your } \\
\text { mental and } \\
\text { physical } \\
\text { abilities } \\
\text { changed } \\
\text { with age? } \\
\text { If so, how? }\end{array}$ & 18. $\hat{=}$ & $20 . \stackrel{\wedge}{=}$ & 20. $\triangleq$ \\
\hline $\begin{array}{l}25 . \text { How do you } \\
\text { handle these } \\
\text { changes? }\end{array}$ & 28. $\triangleq$ & 19. $\triangleq$ & 21. $\triangleq$ & 21. $\hat{=}$ \\
\hline & & & & $\begin{array}{l}\text { 22. The literature } \\
\text { often } \\
\text { discusses } \\
\text { the concept } \\
\text { of } \\
\text { 'successful } \\
\text { ageing' - } \\
\text { what do } \\
\text { you } \\
\text { understand } \\
\text { by this } \\
\text { concept? } \\
\text { What do } \\
\text { you link to } \\
\text { this } \\
\text { concept? }\end{array}$ \\
\hline & & & & $\begin{array}{l}\text { 23. Would you } \\
\text { call } \\
\text { yourself as } \\
\text { successfull } \\
\text { y ageing? } \\
\end{array}$ \\
\hline & $\begin{array}{l}\text { 29. Do you } \\
\text { think that } \\
\text { the } \\
\text { physical } \\
\text { and mental } \\
\text { changes } \\
\text { related to } \\
\text { ageing can } \\
\text { be } \\
\text { stopped/slo } \\
\text { wed } \\
\text { down/contr } \\
\text { olled? If } \\
\text { so, how? If } \\
\text { not, why } \\
\text { not? }\end{array}$ & 20. $\triangleq$ & $22 . \stackrel{\wedge}{=}$ & 24. $\triangleq$ \\
\hline & $\begin{array}{l}\text { 30. Did older } \\
\text { age have an } \\
\text { impact on } \\
\text { your } \\
\text { understandi } \\
\text { ng of your } \\
\text { own }\end{array}$ & 21. $\triangleq$ & 23. $\triangleq$ & 25. $\triangleq$ \\
\hline
\end{tabular}




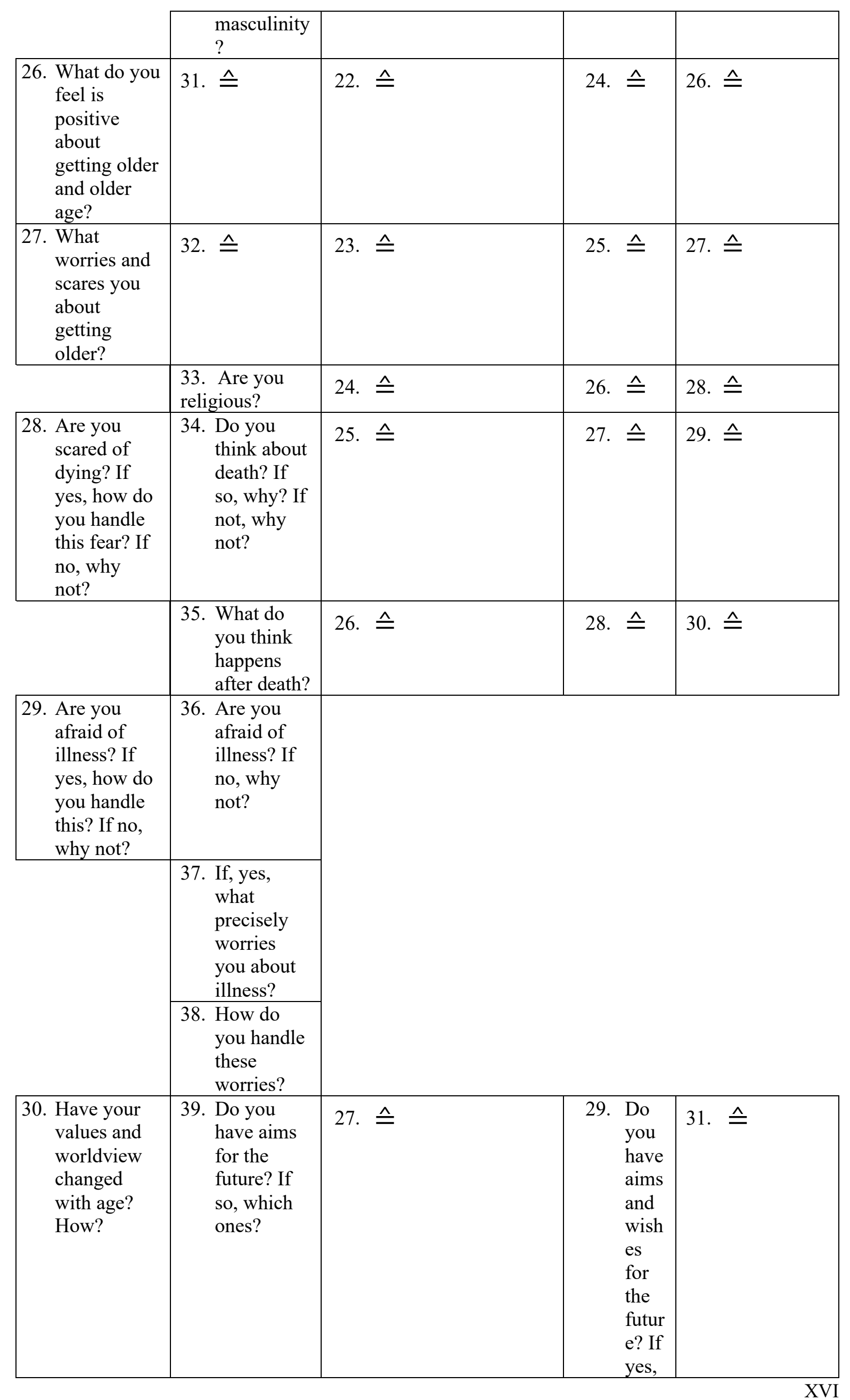




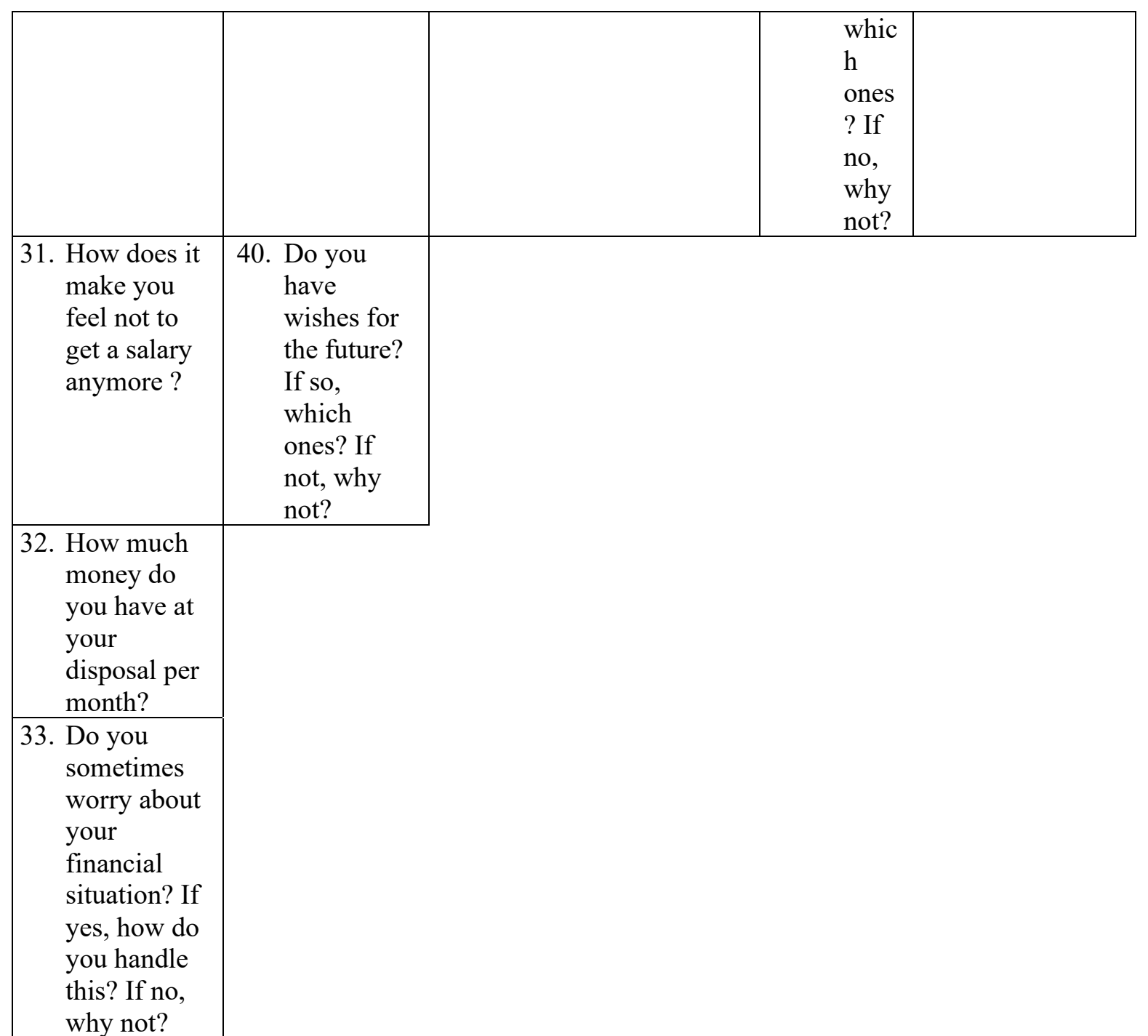

why not?

28. Have your values and worldviews changed

with age? If so, how? If not, why not?

41. Have your

values and

worldviews

changed

with age?

If so, how?

If not, why

not?

42. How does

it make you

feel not to

get a salary

anymore?

43. Do you

sometimes

worry

about your

financial

situation?

If yes, how

do you

handle

30. $\stackrel{\wedge}{=}$

32. 스 


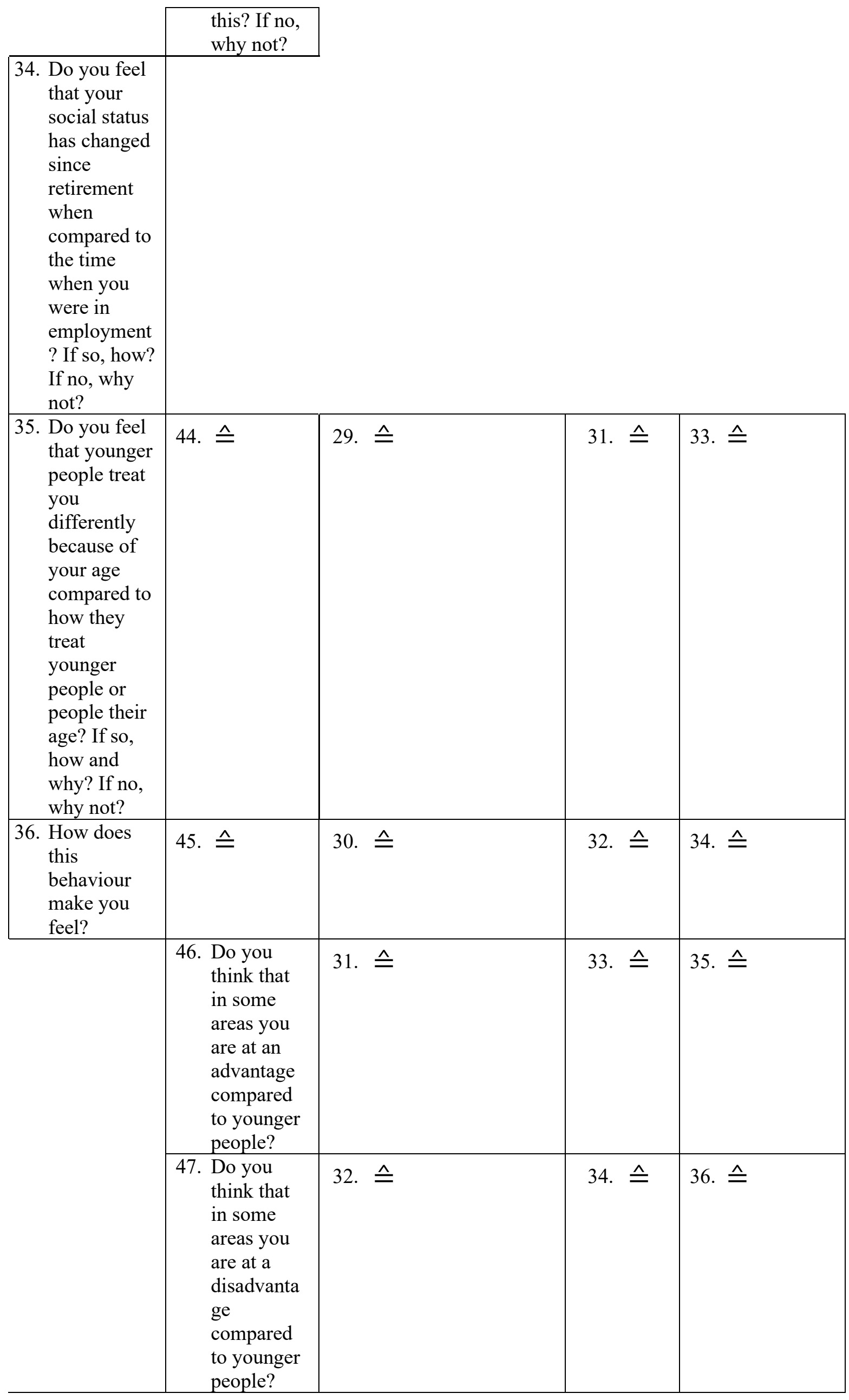




\begin{tabular}{|c|c|c|c|c|}
\hline $\begin{array}{l}\text { 37. How do you } \\
\text { experience } \\
\text { the } \\
\text { depiction of } \\
\text { older people } \\
\text { in the } \\
\text { media, e.g. } \\
\text { ads, films or } \\
\text { on TV? }\end{array}$ & $\begin{array}{l}\text { 48. } \\
\text { (supported } \\
\text { by visuals) }\end{array}$ & & & \\
\hline $\begin{array}{l}\text { 38. Do you } \\
\text { think that } \\
\text { there are } \\
\text { differences } \\
\text { in the } \\
\text { depiction of } \\
\text { older } \\
\text { women and } \\
\text { older men in } \\
\text { the media? } \\
\text { If so, what } \\
\text { differences } \\
\text { are there? If } \\
\text { no, why do } \\
\text { you think } \\
\text { that is the } \\
\text { case? }\end{array}$ & & & & \\
\hline \multirow[t]{2}{*}{$\begin{array}{l}\text { 39. Do you feel } \\
\text { represented } \\
\text { by the } \\
\text { depiction of } \\
\text { older men in } \\
\text { the media? } \\
\text { If so, why? } \\
\text { If not, why } \\
\text { not? }\end{array}$} & $\begin{array}{l}\text { 49. To what } \\
\text { extent does } \\
\text { the media } \\
\text { representati } \\
\text { on of older } \\
\text { men reflect } \\
\text { your own } \\
\text { experiences } \\
\text { as an older } \\
\text { man? }\end{array}$ & & & \\
\hline & $\begin{array}{l}\text { 50. Do you } \\
\text { think } \\
\text { ageing is } \\
\text { more } \\
\text { difficult for } \\
\text { men or for } \\
\text { women? } \\
\text { Why? }\end{array}$ & 33. $\triangleq$ & $35 . \stackrel{ }{=}$ & 37. 스 \\
\hline $\begin{array}{l}\text { 40. How would } \\
\text { you describe } \\
\text { the attitude } \\
\text { of our } \\
\text { society to } \\
\text { older age } \\
\text { and ageing? }\end{array}$ & 51. $\triangleq$ & 34. $\hat{=}$ & 36. $\triangleq$ & 38. 스 \\
\hline $\begin{array}{l}\text { 41. How does } \\
\text { this attitude } \\
\text { or these } \\
\text { attitudes } \\
\text { make you } \\
\text { feel? }\end{array}$ & 52. $\hat{=}$ & $\begin{array}{l}\text { 35. How does this } \\
\text { attitude (s) make you } \\
\text { feel and do you } \\
\text { handle it/them? }\end{array}$ & 37. $\triangleq$ & 39. 스 \\
\hline
\end{tabular}




\begin{tabular}{|c|c|c|c|c|}
\hline $\begin{array}{l}\text { 42. How do you } \\
\text { handle this } \\
\text { attitude or } \\
\text { these } \\
\text { attitudes? }\end{array}$ & 53. $\triangleq$ & & & \\
\hline \multirow[t]{6}{*}{$\begin{array}{l}\text { 43. What do you } \\
\text { think is/are } \\
\text { this/these } \\
\text { attitude (s) } \\
\text { or these } \\
\text { attitudes } \\
\text { based on? }\end{array}$} & 54. $\triangleq$ & 36. $\triangleq$ & $38 . \stackrel{ }{=}$ & $\begin{array}{l}\text { 40. Where and } \\
\text { how, do } \\
\text { you think, } \\
\text { are these } \\
\text { understand } \\
\text { ings of } \\
\text { ageing } \\
\text { transmitted } \\
?\end{array}$ \\
\hline & & $\begin{array}{l}\text { 37. To what extent does } \\
\text { the media } \\
\text { representation of } \\
\text { ageing have an } \\
\text { influence on socio- } \\
\text { cultural views of } \\
\text { ageing? }\end{array}$ & 39. $\triangleq$ & \\
\hline & & $\begin{array}{l}\text { 38. To what extent does } \\
\text { the media } \\
\text { representation of } \\
\text { older men reflect } \\
\text { your own experiences } \\
\text { as an older man? }\end{array}$ & 40. $\hat{=}$ & \\
\hline & $\begin{array}{l}\text { 55. Have you } \\
\text { ever spent } \\
\text { more than } \\
\text { 4-6 weeks } \\
\text { abroad? If } \\
\text { so, where } \\
\text { and for } \\
\text { how long? }\end{array}$ & & & \\
\hline & $\begin{array}{l}\text { 56. In your } \\
\text { opinion or } \\
\text { experience, } \\
\text { are their } \\
\text { cultural } \\
\text { differences } \\
\text { in the } \\
\text { perception } \\
\text { of ageing? } \\
\text { If so, why? } \\
\text { If not, why } \\
\text { not? }\end{array}$ & 39.스 & 41. $\triangleq$ & 41. $\hat{=}$ \\
\hline & $\begin{array}{l}\text { 57. If yes, what } \\
\text { are these } \\
\text { differences } \\
\text { ? }\end{array}$ & & & \\
\hline $\begin{array}{l}\text { 44. To finish } \\
\text { off, now } \\
\text { some } \\
\text { demographi } \\
\text { c questions. } \\
\text { What is your } \\
\text { nationality? } \\
\end{array}$ & $\begin{array}{l}\text { 58. To finish } \\
\text { off, now } \\
\text { some } \\
\text { demograph } \\
\text { ic } \\
\text { questions. } \\
\text { What is }\end{array}$ & & & \\
\hline
\end{tabular}




\begin{tabular}{|l|l|}
\hline $\begin{array}{l}\text { Have you } \\
\text { always lived }\end{array}$ & $\begin{array}{l}\text { your } \\
\text { nationality? }\end{array}$ \\
in Germany? & Have you \\
always \\
Have you & lived in \\
ever spent a & Germany? \\
more & Where do \\
extended & you live at \\
period of & the \\
time in & moment? \\
another & Have you \\
country? If & always \\
so, where & lived in ...? \\
and for how & Do you live \\
long? Where & in your \\
do you live & own \\
at the & property or \\
moment? & do you live \\
Have you & in a rented \\
always lived & property? \\
in ...? Do & \\
you live in & \\
your own & \\
property or & \\
do you live & \\
in rented & \\
property? & \\
\hline
\end{tabular}


Appendix C Google images used in interview have been redacted for copyright reasons. 
D Concept-Map Interviews

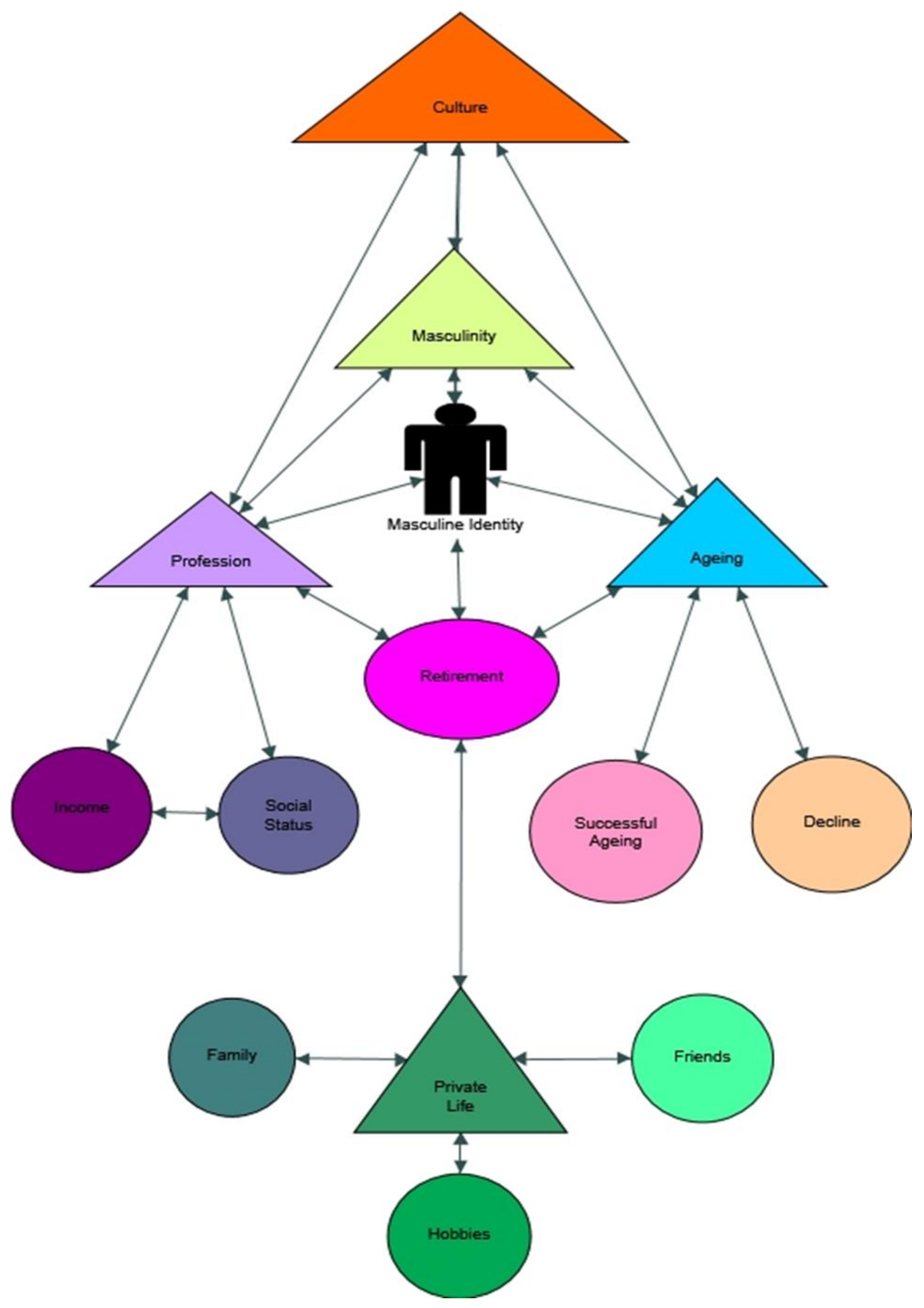




\section{E Reflections on Interviewing Process}

\section{Pilot interview, 11.06.2017:}

- Being too close to the interviewee is challenging since you might have a different recollection of reported experiences \& lack of neutrality

- On the other hand: including my Dad in my research has created a specific rapport between us, he is taking much more interest in my research since he much better understands what I am doing and interested in now

- Questions were often too indirectly phrased: instead of asking what I am interested in I circled around it $\rightarrow$ possibly culturally based for two reasons: 1 . It is considered impolite to ask too directly 2 . Research is often carried out to test hypothesis rather than informed by a real interest in the experiences of the respondents and therefore the questions asked are not necessarily what one is interested in

- I realised that I had not focused on cultural differences in the understanding of ageing at all although this is a major element in my research

- More focus on masculinity and the effect of age on the understanding of oneself as a man = identity

- More focus on the effect of being a pensioner and lack of employment on the understanding of oneself = identity

- More focus on death/illness and concrete issues/understandings related to it such as faith and religious orientation

- Realised that questions about media portrayal of ageing were based on my own media usage but most people do neither watch as much nor the same content as me so there is a need for more input from my side since it otherwise the discussion stays too vague and hypothetical $\rightarrow$ will include visuals to elicit and aid responses

- Informed consent form a little too complicated $\rightarrow$ simplified it by reducing the options

- Couldn't get myself to ask questions about sexual activity and will probably exclude this topic

- Overall: relatively happy with my performance, more practice needed in probing, breaks should be taken, the interview will take about 2 hours

\section{Summer School June 2017}

- Realised my own ageism: never even occurred to me to ask participants about future wishes, aims ...

- Focus on technological orientation/knowledge of older people

\section{Interview \#1 (Rosenfeld, 17.07.2017, 1 hour 47 min)}

- Much too many questions $\rightarrow$ asking 58 questions is a strain on me and the participants and constrains me too much particularly when probing as well

- A more flexible approach will allow me to gain more depth and follow my participants in their line of thought 
- More direct and much fewer questions will enable a more intense reflection on the topics that interest me

- I felt that I need to take myself back more while still allowing for rapport building $\rightarrow$ I said too much as a reaction to what Burkhard Rosenfeld said to establish rapport and trust but at the moment there is sometimes too much input from me and I need to work on finding a better balance between my reactions and what my respondents say

- The media part is still highly unsatisfactory: showing the participant pictures only leads to them choosing which one represents him/her best which was not the intention $\rightarrow$ will remove them for the next interview and change the order of questions to first ask about attitude to age in society and then where does this come from and then bring in media

- Technical problems: no possibility to check recording after the interview: take headphones with me

- Need to ask about upbringing/childhood experiences as influential for the view of masculinity??

- Army service?

- Informed consent needs to be further simplified

\section{Interview \# 2 (Schotten, 18.07.2017, 1 hour 35 min but there were some interruptions)}

- There was only one day between the first and the second interview which is too short to act on the realisations from the previous interview

- Nevertheless, the inclusion of a question on the influence of upbringing on the perception of masculinity worked well while I decided against a specific question about the army service since it will come up in their reflection on their professional development and since I did not extend the number of questions

- Prof. Dr Schotten totally different character than Burkard Rosenfeld $\rightarrow$ much less talkative and more to the point and precise $\rightarrow$ interview much shorter

- Nevertheless, the interview was not less informative and probing and keeping in control of the interview was much easier

- I tried to rely more on the thematic units summarised in the mind map than on ticking off the 60 questions and to more go with the flow of conversation while not saying too much myself $\rightarrow$ worked, but I still need more practice since I am always worried that I forget to tackle a particularly significant question which stresses me which in turn might impact the interview situation negatively since it leads me to be more interested in asking all my questions than in what my interviewees say and in probing for more detail

- Not using the visual input and instead asking some more general questions about the representation of ageing and older people led naturally to a discussion of media representation of older people but also of other cultural agents crucial in the dissemination of socio-cultural narratives, as I had hoped, and following this by questions focusing on cultural differences in the understanding of ageing was a good idea and worked well

- Felt somehow more intimidated by Prof. Dr Schotten than by Burkard Rosenfeld $\rightarrow$ possibly because of the titles and his academic work so felt he is more in a position to evaluate me and my work $\rightarrow$ rapport perhaps a little weaker than in the previous interview which might also be a reason for the shorter time the interview took 
- Realised that in both interviews talking about money makes everybody feel uncomfortable $\rightarrow$ socio-cultural background in which talking about money is considered rude + topic will come up anyways in the discussion about lifestyle $\rightarrow$ explicit questions could be excluded

\section{Reflection Interview \# 3 (Gerd Becker, 15.12.2017, 2 hours)}

- A more extended period of time between the last interview and this one was beneficial:

- transcription process enabled me to reflect on the usefulness of questions asked and on the effectiveness of my probing

- continued and intensified literature review and starting with writing up my research made me realise that I needed some focus on chronological time and subjective perceptions of age

- more focus and confidence due to more practice since in the meantime (28.11.2017) I had conducted another interview which in the end I excluded since the participant did not fit the hegemonic masculinity ideal as well as the other participants: interview was very short and participant quite unwillingly to reflect on ageing which probed me to reflect on the reasons for this and led me to the realisation that besides possibly being the result of character, it might have also been since the participant did predominantly not match hegemonic masculinity standards which put him in a more defensive position and also meant that he could not relate as well as my other participants to the questions I asked $\rightarrow$ experience aided me immensely since it forced me to intensely think about the aim of my interviews and the selection of the participants and it thereby, in my view, supported a more consistent and focused research approach

- Excellent rapport between me and the interviewee $\rightarrow$ similar character, outgoing, reflected and open personality

- However, this also meant that the conversation veered off focus and I was forced to approach the interview much more flexibly than in the previous ones $\rightarrow$ although I found this rather stressful, mostly basing the interview on thematic units rather than proceeding from one interview question to the next, worked astonishingly well which increased my confidence in interviewing

- Data was so rich and also in many ways contrary to interviews conducted before that I am considering not conducting any further interviews but need to think about this a little more and also discuss this issue with my supervisors

\section{Reflection Interview \# 4 (Dr Lars Bühring, 31.07.2018, 1 hour 16 min.)}

- Although during the pilot interview I felt that being too close to the interviewee and knowing him too well can be a problem I, after thinking about it intensely, decided to, after all, conduct my last interview with my father because:

- the ageing process of my parents has been the main reason for my research focus, and as such it seemed logical to me to include, at least, my Dad in the research since I really wanted to find out more about his life-course narrative(s), his identity construction and any changes to this identity construction in older age $\rightarrow$ in the end, I have to admit that much of the interest in ageing and related aspects was and 
is motivated by getting to know and understanding my parents and particularly my father better

- my father has taken a vital interest in the research process, and I wanted him to be part of my thesis because I believed it could be beneficial for him, me and also for our relationship which, after my mother passed away, has slowly improved markedly

- my father used to fit the hegemonic masculinity ideal in many ways, and I believed he could provide vital insights into his identity construction in older age - including my father in the interviews could aid me in focusing on understanding his and the other interviewees' lifeworld and life course narratives rather than evaluating and categorising them, as is typical for the German research approach since it is essential to me to capture my interviewees life worlds adequately and in a way which is respectful and which my participants can relate to

- Since there was a time gap between the last and this interview and I had written up my literature review in the meantime, I included explicit questions on the successful ageing paradigm which before I had instead, in the German manner, circled around the topic of successful ageing than explicitly asking for it $\rightarrow$ worked but no significant difference to my previous interviews

- I also decided to approach the questions regarding the media representation slightly differently and take here contrarily a less explicit approach but instead ask about where my respondent thinks the socio-cultural understanding of ageing comes from to allow for more flexibility in the answers $\rightarrow$ worked well

- My father was very focused and precise in his answers which is reflected in the relatively short time the interview took and also in the clarity both in speech and content of my father's answers $\rightarrow$ clearly a result of our many previous discussions on the topics covered and his wish to provide me with valuable input and also his rather rational and controlled personality

- Nonetheless, I believe that his answers reflect his views and are not aimed at pleasing me $\rightarrow$ I had repeatedly discussed with my father that the aim of my research is not to confirm preconceived ideas of mine but to really gain a better understanding of the lives of my older male participants

- Although it somehow sometimes felt a bit awkward I think for both of us to be in a situation in which we covered topics we had discussed before in a much less structured and informal way, but overall the interview went well, and I believe it was a fruitful and enriching experience for both of us 


\section{Name}

Ageing as Decline

\section{Description}

Interviewee responses that relate to the socio-cultural narrative of ageing as decline

Sub-aspects: mental decline, physical decline, identity, finiteness of being/death

\begin{tabular}{|c|c|}
\hline $\begin{array}{l}\text { Control and } \\
\text { Autonomy }\end{array}$ & $\begin{array}{l}\text { Interviewee responses that relate to aspects of } \\
\text { personal control and autonomy and their significance } \\
\text { for the interviewees } \\
\text { Sub-aspects: Autonomy and control in professional } \\
\text { context, personal autonomy and control, autonomy } \\
\text { and control of others, identity }\end{array}$ \\
\hline $\begin{array}{l}\text { Society, Culture and } \\
\text { Media }\end{array}$ & $\begin{array}{l}\text { Interviewee responses that relate to socio-cultural } \\
\text { narratives of ageing } \\
\text { Sub-aspects: Corporate culture, German culture \& } \\
\text { ageing, other cultures \& ageing }\end{array}$ \\
\hline $\begin{array}{l}\text { Generational } \\
\text { Conflict }\end{array}$ & $\begin{array}{l}\text { Interviewee responses that relate to the relationship } \\
\text { between the older and the younger generation } \\
\text { Sub-aspects: Younger generation, older generation }\end{array}$ \\
\hline $\begin{array}{l}\text { Life Course } \\
\text { Narratives }\end{array}$ & $\begin{array}{l}\text { Ruled out in detailed coding since all aspects below } \\
\text { and above are part of life-course narratives }\end{array}$ \\
\hline $\begin{array}{l}\text { Masculinity and } \\
\text { Femininity }\end{array}$ & $\begin{array}{l}\text { Interviewee responses that relate to the personal and } \\
\text { socio-cultural understanding of masculinity } \\
\text { Sub-aspects: Masculinity, femininity, father, mother, } \\
\text { parents' relationship, mentor/role model, marriage, } \\
\text { negative role model }\end{array}$ \\
\hline Successful Ageing & $\begin{array}{l}\text { Interviewee responses that relate to the socio-cultural } \\
\text { narratives of successful ageing } \\
\text { Sub-aspects: Current occupation (professional), } \\
\text { current occupation (personal), identity, } \\
\text { retirement/older age }\end{array}$ \\
\hline
\end{tabular}




\section{G Interview \& Translated Extracts Burkhard Rosenfeld}

$1 \quad[\ldots]$

2 LB: Dann fangen wir direkt mal an. Was bedeutet Männlichkeit für Sie? (Lange Pause)

...What does masculinity mean to you?

3 Burkhard Rosenfeld: Männlichkeit bedeutet für mich die Fähigkeit an der Fortpflanzung

4 teilzunehmen und dabei eine wichtige Rolle zu spielen. Mit Männlichkeit ist für mich auch

5 damit verbunden dieses traditionelle Männer- Frauenbild. Das eben der Mann bei den

6 früheren Höhlenmenschen auf die Jagd geht und die Frau eben für den Teil, der für die

7 Familie für die Versorgung der Familie zuständig ist. Der Mann der Familie Schutz bietet

8 und die Frau der Familie Emotionen, Gefühl bietet.

To me masculinity means to have the ability to take part in the reproduction and play a crucial role in it. Masculinity is also linked to this traditional understanding of the male respectively female role. That is that the men of the cave people went hunting and the women were responsible for the part, which took care of the family. The man provides the family with protection and the woman provides emotions and feelings.

$9 \quad[\ldots]$

10 LB: Sie haben eben gesagt dass Sie so große Konzerne sind nicht so Ihre Sache. Warum?

You said that big companies are not your thing? Why?

11 Burkhard Rosenfeld: Ich bin kein Politiker. Ich bin rechtwinklig konstruiert. Das heißt ich

12 kann nur, ich kann nur die Wahrheit oder es sein lassen. Die liebe ich oder ich hasse

13 Hinterhältigkeit und all das findet sich im täglichen Geschäft der Großkonzerne wieder.

14 Auch wenn man zwischendurch Menschen begegnet die anders geartet sind ... aber das ist

15 sie das ist die Kultur der Großkonzerne, allen die ich bisher kennengelernt habe und ich

16 möchte, ich möchte gestaltend wirken, ich möchte, ich möchte meine Entscheidungen allein

17 treffen können - wobei ich generell Team orientiert bin aber ich möchte nicht Handlanger

18 sein - es sei denn ich bin in einer Lernphase wo ich etwas lernen muss - ich möchte den Weg

19 den ich wähle zur Lösung von Problemen... der Weg den ich einschlage zur Lösung selbst

20 wählen. Bei Indus habe ich den Weg gewählt über die Menschen. Ich habe meine Arbeit

21 erledigt, indem ich Menschen gefunden habe den ich Vertrauen entgegengebracht habe und

22 die mir vertraut haben und die ich unterstützt habe wenn sie in Not waren, auch in ganz 
1 schwierigen Situationen, zu denen ich aber auch offen war wenn es notwendig war harte

2 Entscheidungen zu treffen.

I am not a politician. I am constructed rectangularly. That means, I can only, I can only do truth or not do it. I love or I hate underhandedness und all that can be found in the daily business of big corporations...this is the culture of multinationals.

3 LB: Das sind praktisch schon, sind meine nächste Frage nämlich: Was hat Ihnen besonders

$4 \quad$ Freude gemacht bei der Indus und was nicht so?

...What did you particularly enjoy at Indus?

5 Burkhard Rosenfeld: Bei der Indus hat mir Freude gemacht dass ich von Anfang an allein

6 Entscheidungen treffen konnte. Bei der Indus hat mir Freude gemacht dass ich in den

7 Bereichen in denen ich wenig Kompetenz habe und in denen ich auch kein so großes

8 Interesse habe gute Gesprächspartner hatte auf deren Votum ich mich verlassen konnte. Bei

9 Indus hat mir Freude gemacht die Art wie wir zusammengearbeitet haben. Wenn ich an den

10 Vorstandsvorsitzender und Gründer denke, der ein sehr großes Geltungsbedürfnis hatte und

11 dessen Spuren ich nach meinem beruflichen Ende mehrfach begegnet bin, also Menschen

12 die mit ihm früher zu tun hatten, dann musste ich denen immer sagen die Zusammenarbeit

13 war immer fair und es gab keinen Fall wo ich mich nicht verlassen konnte, wo ich mich nicht

14 auch auf den Vorsitzenden verlassen konnte. Die Leute haben da nur immer ungläubig mit

15 dem Kopf geschüttelt weil sie andere Erfahrungen gemacht hatten. Aber das hat mir Freude

16 gemacht. Und wenn das nicht gewesen wäre dann hätte ich wieder meine Konsequenz 17 gezogen.

At Indus I enjoyed that from the beginning I could make decisions autonomously. At Indus I enjoyed that in areas in which I had little competence and which I also was not that interested I had good conversation partners on whose vote I could count. At Indus I enjoyed the way we worked together...the corporation was always fair and there was no case in which I couldn't count, in which I couldn't count on the chairman.

$18 \quad[\ldots]$

19 LB: Ja das ist schon befriedigend, denke ich ...? Gab es denn in der Zeit bei Indus auch

20 Dinge die sie gar nicht gerne gemacht haben, die gegen ihre Überzeugung gegangen wären

21 aber wo sie wirklich dran zu arbeiten hatten? 
...Were there any things during your time at Indus that you didn't enjoy so much, that were against your believes, ...

1 Burkhard Rosenfeld: Nein. Nein weil ich ... Ich lebte immer in der Gewissheit dass ich

2 mich auf meine beiden Kollegen verlassen kann. Als ihr Vater einmal da irgendwo im Schnee

3 versunken war und an einem Termin nicht teilnehmen konnte, musste ich als Ingenieur

4 einen 100 Millionen Kredit unterschreiben. Ohne es studiert zu haben, ohne das gelesen zu

5 haben. Und natürlich kannte ich den Hintergrund und natürlich kannte ich das Konzept und

6 so weiter.

...I always lived in the certainty that I could count on my colleagues...

$7 \quad[\ldots]$

8 LB: Wenn Sie zurückdenken hatte Ihre Position speziell in der Indus einen Einfluss darauf

9 wie Außenstehende Sie gesehen haben, haben Sie den Eindruck gehabt?

When you think back, did your position at Indus have an impact on how outsiders viewed you, did you have the impression?

10 Burkhard Rosenfeld: Ich habe, was verstehen Sie unter Außenstehende?

What do you mean by outsiders?

11 LB: Eher nicht sehr Nahestehende sondern eher Leute, die man, Bekannte, ...

Rather not people who are close but more acquaintances...

12 Burkhard Rosenfeld: Außerhalb der Firma?

Outside of the company?

13 LB: Ja, außerhalb der Firma, die aber ...mit denen Sie dann zu tun hatten.

Yes, outside of the company but...people you had contact with.

14 Burkhard Rosenfeld: Ich habe immer oder ich liebe, ich liebe Diskretion und mag den

15 Auftritt nach außen nicht. Und deswegen haben ganz viele Leute gar nicht mitbekommen

16 was ich überhaupt tue. Mein Nachbar - ich habe das benachbarte Haus gekauft als ich das

17 zweite Mal geheiratet habe, das Haus - und das Haus gehörte meinem Nachbarn schon lange,

18 der war damals Vorstand von Bayern in Italien, Vorstandsvorsitzender von Bayer in Italien. 
1 Da kam er zurück bei seiner Pensionierung und so - der wusste bis vor ein oder zwei Jahren

2 noch gar nicht was ich überhaupt beruflich mache.

I always have, or I love, I love discretion, and I don't like public appearance. And that is why many people did not even know what I was doing...

$3 \quad[\ldots]$

$4 \quad$ LB: Weil Sie sich über andere Dinge identifizieren und nicht über berufliche Position?

Because you identify through other things and not through your professional position?

5 Burkhard Rosenfeld: Also Ich identifiziere mich darüber dass mich Mitarbeiter die ich

6 vor ... bis 45 Jahre zurück, dass sich Mitarbeiter bei mir melden um mir zum Geburtstag

7 gratulieren oder um Rat fragen oder so etwas. Das motiviert mich und mich motiviert das

8 ein Geschäftsführer den ich gekündigt habe ziemlich am Anfang von Indus, das ich mit dem

9 fast freundschaftlich verbunden bin. Mich motiviert... mich motiviert dass ich bei mir bleiben

10 kann und es in meinem Berufsleben außer bei der einen Position mit dem Freund das 'Du'

11 vermeiden konnte, das finde ich auch toll. Ihr Vater, ihr Vater und ich wir sind uns so nahe

12 aber wir duzen uns nicht - das ist der Dr Bühring und ich bin der Herr Rosenfeld. Und das

13 ist für mich wichtig. Mir ist unwichtig das ich da einen Auftritt habe oder. Ich habe auch

14 immer vermieden große Autos. Ich habe immer einen Fünfer BMW gehabt.

Well, I identify myself by the fact that employees from as long as 45 years ago contact me to wish me Happy Birthday or to ask for advice or something like that. That motivates me, or it motivates me that a managing director I had dismissed quite at the beginning of my time at Indus, that I maintain nearly friendly contacts with him. It motivates me; I am motivated by the fact that I could stay true to myself...

16 LB: Ja, da sind wir alle nicht vor gefeit. Wie waren denn so Ihre Prioritäten während ihres

17 Berufslebens? An erster Stelle, an oberster Stelle, mittlere, Sie wissen schon worauf das 18 hinausgeht... natürlich.

...What were your priorities during your professional career?...

19 Burkhard Rosenfeld: Ich habe meine Familie vernachlässigt. Ich habe meine Familie 20 vernachlässigt, ich habe meine Familie nicht willentlich vernachlässigt. Ich habe kein

21 Wochenende gearbeitet. Außer ich war am Verreisen. Aber am Wochenende musste ich 
natürlich Sport machen. Und wenn meine Frau eben den Sport, das war damals Tennis, nicht mitmacht ist sie selber schuld. Als ich sie kennenlernte spielte sie Tennis. Ich war geprägt durch meine Herkunft. Ich bin geboren Anfang 1941 in Königsberg Ostpreußen, habe die zwei großen Luftangriffe auf Königsberg im Luftschutzkeller als Kleinkind mitgemacht und 1944 im August wurde unser Haus zerstört. Und da ist meine Mutter, durfte meine Mutter raus aus Königsberg und wir mussten nach Westpreußen und hat sich dann relativ schnell auch in Bewegung gesetzt Richtung Süden. Und dann irgendwann ging es zu Ende da waren wir im Allgäu und dann bin ich in kargen Verhältnissen aufgewachsen. Meine Mutter hat 19und....48, glaube ich, 48 hat sie erfahren dass mein Vater im Februar 45 nahe ihrer Heimatstadt, ihrer Geburtsstadt gefallen ist, wo er Soldat war. So hat die, meine Mutter, mit 29 war der Krieg zu Ende mit 32 hat sie erfahren, dass sie Witwe ist und da hat sie zwei so Jungs und muss dann mit großen Entbehrungen die Kinder aufziehen. Das hat mich geprägt dass ich eben so schnell wie möglich unabhängig werden wollte, ich wollte also wirklich mein Leben selbst verdienen können. Und ich hab dann halt nicht nur mein Leben selbst verdienen können sondern ich habe ein bisschen zusammen mit meinem Bruder für meine Mutter sorgen können, die ein unheimlich toller Mensch war. Und das hat mich natürlich geprägt - wieder zurück bei der Frage, das hat mich geprägt. Ich sorge für die Sicherheit der Familie. Und meine Frau für die Familie selbst, für die Wärme der Familie. Und das führt halt dazu, dass ich zusammen mit meiner sehr leicht aus den Vorhergegangen ableitbaren Einsatzbereitschaft für eine Aufgabe, das die Familie, nach heutigen Maßstäben viel zu kurz kommen ließ.

I neglected my family. I neglected my family; I did not willingly neglect my family. I didn't work at weekends except when I travelled. But at weekends I had to do sports. And if my wife didn't join me in it, at that time it was tennis; then it is her own fault.... I was influenced by my family background. ...So she had, my mother had, with 29 the war ended and with 32 she found out that she is a widow and there she had two boys and had under great privations to bring up these children. That influenced me so that I wanted to become independent as soon as possible; I really wanted to earn my own living. And then could not only earn my own living but I could also together with my brother take care of my mother, who was a fantastic person. And that obviously influenced me ... I take care of the protection of my family. And my wife takes care of the family itself, of the warmth of the family... the family got a raw deal according to current standards.

22 LB: Nach Ihren heutigen Maßstäben?

According to your current standards? 
Burkhard Rosenfeld: Nein, nach allgemeinen Maßstäben.

No, according to general standards.

\section{[...]}

LB: Das finde ich interessant weil sie ja am Anfang auch gesagt haben dass sie an diese traditionelle Rollenverteilung glauben. Der Mann ist derjenige der das Brot verdient. Das ist ja, ich meine, das ist ja auch nach wie vor eigentlich durchaus verbreitet. Es traut sich halt niemand das so auch zu benennen. Spricht ja auch gar nichts dagegen. War das auch bei ihnen so, hat ihre Frau gearbeitet oder ...?

I find that interesting since you said at the beginning that you believe in this traditional role division...Was that also the case with you, did your wife work, or...?

Nein, das war das Dilemma auch. Meine Frau hat gesehen wie ich eben anpacke und wie auch, auch mich einsetzte und zum großen Teil auch begeistert war von den Dingen die ich gemacht habe. Sie hat nach dem Abitur auch in München zu studieren begonnen, neue Sprachen Englisch, Französisch, Spanisch, hat aber nach kurzer Zeit hat sie festgestellt, dass das nicht ihr Fall ist es und ist dann an die Sprachenschule gegangen. Gab's in, neben dem der Universität in München und hat sich dort zur, das war auch ihr Ziel, zur Übersetzerin ausbilden zu lassen aber vorher hat sie mich dann Kennengelernt. Dann hat sie das abgebrochen mit dem Abschluss einer Fremdsprachenkorrespondentin. Hat ganz kurz am Anfang oder, ja, ganz kurz am Anfang, ganz am Anfang etwas gearbeitet dann nicht mehr. Ich wollte es auch nicht aber meine Frau, meine Frau wollte das im Grunde auch nicht weil sie war - das hätte sie unterfordert. Sie hat es später, sie hat später, na was sage ich, das war, das war als meine Frau 46 Jahre alt war, 45/46 da hat sie eigentlich zu dem gefunden was ihr Spaß gemacht hat. Sie war interessiert an Kunst und verstand ne ganze Menge davon. Sie war dann Mitarbeiterin, ja Redakteurin, ja, einer damals sehr bekannten Kunstzeitschrift, Kunst - und Antiquitäten, hieß die. Da war sie tätig und ihre Aufgabe war eben auch zu den Messen zu fahren und Berichte drüber zu schreiben. Das hat sie dann als wir ...das hat sie spät dann ... Kunst und Antiquitäten wurde später eingestellt sie war zu toll und zu teuer. Übriggeblieben ist die Weltkunst, die gehört ja zum Zeitverlagskonzern. Und dann hat meine Frau das Gleiche gemacht in der FAZ. Hat meine Frau in der FAZ Berichte geschrieben vom Kunstmarkt. War aber dort geachtet als freie Mitarbeiterin aber bekannt. Und das wäre es eigentlich gewesen. Das hat sie eben erst sehr spät gefunden. Als sie unter Druck gesetzt wurde. Ich hab gesagt, mach das, mach dies - nee, nee... Das hat ihr richtig Spaß gemacht. 
Not that was the dilemma. My wife saw how I tackle things and how, how I commit myself and how I was also, to a large part, enthusiastic about the things I did...My wife wrote articles about the art market for the FAZ as a freelancer...And that would have been it. But that she found very late. When she was put under pressure. I said do this, do that -yes, yes...That she really enjoyed.

1 LB: Und da war auch ihre Tochter dann auch, hatte ein bestimmtes Alter. Ich meine, das hat

2 ja durchaus was miteinander zu tun. Ich kenne das ja auch von meiner Mutter.

And then there was your daughter; she had a certain age also. I mean, that is somehow linked. I know that from my mother.

3 Burkhard Rosenfeld: Meine Tochter ist sehr komplizierte Mensch, ich habe eine einzige

4 Tochter, ein sehr komplizierter Mensch. Wir haben eine sehr stark gestörte Beziehungen 5 zueinander. Die Erziehung meiner Tochter war für meine Frau - 69 geboren- eine 6 wahnsinnig anstrengende Aufgabe. Es begann damit sie irgendwo in der Stadt waren und 7 sagten lass uns weitergehen. Mutter kommt um die Ecke, kam nicht oder das sie in Essen als 8 sie in die Vorschule ging zu ihrer Lehrerin ging und ihr von ihrem Pausenbrot abgeben soll 9 weil sie keins von zu Hause hatte, was nicht stimmte und so ging das immer weiter. 10 Wahnsinnig kompliziert. Wenn ich verreist war, ich war ja viel in Südamerika und Amerika, 11 und wenn ich verreist war, da spielten sich ab und zu Dramen ab. Unglaubliche Dramen. 12 Meine Tochter hat sehr früh Beziehungen zu jungen Männern gehabt. Wir hatten so ein Haus 13 da konnte man, da gab es, das war in so einer Wohnanlage, eine Edelwohnanlage, da konnte 14 man von außen mit einem Seil auf eine Dachterrasse klettern. wenn man das Seil hatte oben 15 und das hat dann der Gustl gemacht, ohne dass ich es wusste. Ich wusste nur das er 16 übernachtet hat. Und das hat meine Frau ja alles mitbekommen. Ich will es nicht weiter 17 vertiefen. Jedenfalls war eine wahnsinnig anstrengende Zeit. Das war das das Problem der 18 Berufstätigkeit. Meine jetzige Frau hat relativ früh begonnen Pharmazie zu studieren. Und 19 auch diese Zeit in einer Apotheke gemacht mit allem was dazugehört. Dann hat sie ihren 20 ersten Mann kennengelernt und dann hat sie dem zuliebe, der musste dann promovieren, hat 21 sie eben das nicht weitergemacht. Dann ist sie später in die Apotheke gegangen und hat dann jahrzehntelang in der Apotheke gearbeitete als Vorexaminierte also sie war keine fertige Apothekerin habe aber aufgrund ihres Vorexamens sie konnte sie auch Nachtdienst machen. Aber sie hat letztendlich, sie hat letztendlich eben über lange Jahre eben nebenher einen Beruf nicht Vollzeit aber Teilzeit ausgeübt. Das hat ihr eben auch Stärke gegeben, das 26 gab es bei meiner gestorbenen Frau halt nicht. 
But after all, she ${ }^{20}$, she, after all, had additionally ${ }^{21}$ had a job, not full-time but part-time. That somewhat gave her strength, and that did not exist for my late wife ${ }^{22}$.

1 LB: Ich würde gerne weiter fragen aber sonst sitzen wir hier bis um zehn. So jetzt aber

2 endlich zur Rente und dem Alter. Wie war das denn für Sie. Als Sie dann wirklich aufgehört

3 haben zu arbeiten wie hat sich das für Sie gestaltet, war das schwierig...? Gar nicht?

...How was that for you? When you finally stopped working, how was that structured, was that difficult...? Not at all?

4 Burkhard Rosenfeld: Es war wunderbar. Es war wunderbar. Der Rucksack war mir einfach

5 zu schwer geworden, wenn ich es ein bisschen länger gemacht hätte so in der Form dann

6 könnte es sein das ich daran zugrunde gegangen wäre in irgendeiner Form. Ich habe es

7 genossen. Ich habe mir nichts vorgenommen. Ich habe also die Dinge die Normalpensionäre

8 machen, Weltreise, Buch schreiben, studieren, ums Haus rumlaufen, hab ich alles nicht

9 gemacht. Ich hab ausgeschlafen. Und dann hab ich mal Bücher gelesen von Reich-Ranicki

10 gibt es ja diesen Kanon der Deutschen Literatur, die habe ich dann alle gelesen nacheinander.

11 Das konnte vorher nicht, mal ein Buch am Stück lesen. Ich habe es also richtig dick genossen.

12 Meine Frau war so überrascht, der macht ja gar nichts.

That was wonderful. I was wonderful. I had just felt that the backpack ${ }^{23}$ had become too heavy, had I continued a little longer in this way then it could have been that I would have in some way collapsed. I enjoyed it. I had not planned anything. I did therefore not do the things typical retirees do, travelling around the world, writing a book, study, walk around the house, all this I didn't do. I slept in. And then I read books... Before I could not ever read a book in one stretch. I really enormously enjoyed it. My wife was really surprised; he doesn't do anything.

$13[\ldots]$

14 LB: Ach war immer noch was. Sind Sie jetzt in Rente oder machen Sie immer noch ganz 15 viel?

Ach, there still was something. Are you retired, or are you still doing a lot?

\footnotetext{
${ }^{20}$ His second wife

${ }^{21}$ In addition to being a mother and housewife

${ }^{22} \mathrm{He}$ referred here to his first marriage and the fact that his first wife had over long periods of their marriage not worked.

${ }^{23}$ In German a colloquial expression for burden
} 
1 Burkhard Rosenfeld: Ich mache immer noch was. Ich habe immer noch zwei Mandate.

2 Erst wurde ich angesprochen von einer Beteiligungsgesellschaft, die mich kannten, die was

3 Ähnliches machen wie Indus. Und da hab ich da ein Mandat übernommen. Und dann rief

4 mich einer an den ich sehr geschätzt habe, der ist leider Tod inzwischen, das ist der der uns

5 die Simon Gruppe verkauft hat, also die wichtigste Firma im Induskonzern. Der hatte noch

6 eine andere Firma, große Firma, da waren seine Kinder Gesellschafter und auch noch andere,

7 insgesamt zehn. Der bat mich, die wollten einen Beirat aufbauen, und bat mich das zu

8 machen. Dem konnte ich ... Das konnte ich nicht ablehnen. Das habe ich dann und das

9 mache ich immer noch. Und überlege mir jetzt ob ich dieses Jahr aufhöre oder weitermache

10 solange ich gesund bin.

I am now considering if I should stop this year or if I should continue as long as I am healthy.

11 LB: Macht es Ihnen denn Freude?

Are you enjoying it?

12 Burkhard Rosenfeld: Ja, ich habe gemerkt in der Zeit in der ich nur Rentner war, weiss 13 nicht vielleicht waren es auch zwei Jahre, dass meine Gedankenschärfe nachließ, mein

14 Erinnerungsvermögen, das ich öfter mal nach Worten und Sachverhalten suchen musste weil

15 ich sie vergessen habe. Diese permanente Beschäftigung mit diesen Dingen in den Firmen

16 denn ich beschäftige mich mit den Dingen manchmal mehr, manchmal weniger. Das schärft

17 die Sinne sowohl hier(zeigt auf Kopf) als auch Verhalten. Ich lerne Geduld, ich lerne, also

18 gerade das was ... die Schwächen die ich aufweise. Ich lerne die Dinge die ich spontan

19 machen will nicht zu machen. Ich schreibe oft Briefe, die lege ich dann weg. Am Ende leg

20 ich sie nur noch weg. Manchmal schmeiß ich sie weg, manchmal wird dann ein anderer Brief

21 dann geht der raus oder eine Mail oder sowas. Das kann ich jetzt lernen, die zeitliche

22 Inanspruchnahme ist sehr begrenzt und durch das Internet... ist natürlich toll.

Yes, I noticed during the time when I was a retiree, I don't know, maybe it was two years, that my mental sharpness declined, my memory, that I had to more often search for words and facts since I had forgotten them. This being permanently occupied with the matters of these firms as I think about these things sometimes more, sometimes less, that sharpens the senses here (points to the head) as well as in the behaviour. I have learned to be patient, particularly that...the weakness I have. I have learned not to do the things I want to do spontaneously.

23 LB: Haben sich denn generell Ihre Prioritäten verändert? 
Generally, have your priorities changed?

1 Burkhard Rosenfeld: Ja, ja klar. Die Priorität jetzt ist das Leben ist sehr begrenzt, es kann

2 so kurz sein, es kann ein bisschen länger sein. Keine Zeit verschwenden.

Yes, clearly. ...Wasting no time.

$3 \quad$ LB: Mit was würde man denn die Zeit verschwenden?

How would you waste time?

4 Burkhard Rosenfeld: Mit unsinnigen Dingen, mit Ärger, soweit es geht... Und dazu gehört,

5 dazu gehört beispielsweise Gelassenheit. Ich zahle Kirchensteuer und bin immer in der

6 evangelischen Kirche gewesen und der Pfarrer im Nachbarort hat mir letztes Jahr, nicht

7 nur mir den anderen auch ein kleines Zettelchen gegeben, stand drauf Gelassenheit.

8 With meaningless things, with anger, as far as possible...And this includes, for example,

9 placidity...last year the parish priest in the neighbouring community gave me, not only me

10 but all, a small note saying placidness.

$11 \quad$ LB: Aber nicht nur Ihnen?

But he didn't just pass that on to you?

12 Burkhard Rosenfeld: Nein, das war das Thema seiner Predigt. Da habe ich gedacht, passt,

13 das kann ich brauchen.

... Then I thought, fitting, I can use that.

14 LB: Passt, glaube ich fast für jeden. Gelassenheit dass, wenn man das erreichen kann und 15 vor allem auch halten kann. Es gelingt eine gewisse Weile aber dann geht es doch wieder 16 den Bach runter. Ich muss mich gerade hier orientieren, weil Sie sind weiß ja gar nicht

17 richtiger Rentner, Sie sind ja nur sozusagen zwei Drittel Rentner. Aber das ist wunderbar, je 18 unterschiedlicher desto besser. Grundsätzlich aber was mich im Grunde genommen 19 interessiert ist hat das Alter das zunehmende Alter für Sie einen Einfluss darauf wie sie sich 20 wahrnehmen.

... has you age, your increasing age had an influence on how you view yourself?

\section{Burkhard Rosenfeld: Ja.}


Yes.

$1 \quad$ LB: Inwiefern?

In what way?

2 Burkhard Rosenfeld: Ich merke dass, ich merke dass ich, meine Gesundheit, meine

3 gesundheitliche Stabilität zurückgeht. Ich merke dass ich schlecht höre, dass ich schlechter

4 sehe. Ich werde sicherlich auch weicher. Also ich bin, ich bin sicherlich auch in dieser Phase

5 weicher geworden. Die Grundhärte, die Bereitschaft eben zu kämpfen und sich einzusetzen

6 und so weiter und auch mal zuzuschlagen die ist geblieben aber die Barmherzigkeit hat

7 gewonnen.

I have noticed that my health, that the stability of my health is declining. I have noticed that my hearing has worsened that my eyesight has worsened. I am certainly becoming softer. Well, I certainly also have become softer in this phase. A basic toughness, the willingness to fight and to commit yourself and so on and also to strike has remained, but mercifulness has won.

8 LB: Aber es ist nicht so dass sie sich durch das Altern in ihrer Männlichkeit beschnitten 9 fühlen.

But it is not in a way that you feel that your masculinity is impaired?

10 Burkhard Rosenfeld: Nein.

No.

$11[\ldots]$

12 LB: Ja und er hat mir das auch immer... Also ich finde das erstaunlich weil ich halt immer 13 gehört habe sie duzen sich nicht und eigentlich sind sie ja auch gar nicht befreundet. Aber

14 eigentlich gibt es viel engere Freundschaft als die die sie haben über die Jahren gewachsen 15 da, gibt es kaum. Vielleicht nicht wo man am Rhein sitzt und sich das Kölsch hinter die 16 Binde kippt und Dinge tut. Also aber die Nähe die sie zwei haben ist glaube ich kaum mit 17 irgendetwas zu vergleichen. Duzen hin duzen her. Wir haben schon damit angefangen was 18 sie als Einschränkung empfinden vom Alter?

...what do you experience as limitation due to older age? 
1 Burkhard Rosenfeld: Ich empfinde als Einschränkung dass ich wenn ich meine jährlichen

2 Arztbesuche mache das Ende des Besuches, wenn man mir nichts Schlimmes sagt als

3 Freispruch deutet. Früher war das selbstverständlich.

I experience it as a restraint that when I visit the doctor once a year, that at the end if the visit when you don't hear anything bad, it is experienced like an acquittal. In former times, that was a given.

4 LB: Ja. Das heißt Sie setzen sich schon jetzt auch mehr mit der Endlichkeit des Seins 5 auseinander.

Yes. That means you are also thinking more about the finality of being?

6 Burkhard Rosenfeld: Ganz, ganz, ganz konkret. Ganz konkret nicht jetzt in formeller

$7 \quad$ Hinsicht sondern ich genieße jeden Tag und lass mich nicht einschränken in den Dingen die

8 ich gerne tue. Wir haben in Italien ein kleines Häuschen. Da sind wir also ganz viel und

9 trinken, sitzen zu Abend, die Berge und da unten der See und dann haben wir Rotwein. Das

10 mag ungesund sein aber am nächsten Tag strampeln wir ja auf dem Fahrrad - das genieße

11 ich, das genieße ich. Wir genießen Kleinigkeiten. Als wir jetzt zurückgekommen sind, wir

12 sind am Sonntag zurückgekommen, am Samstag, wir genießen das, alles wunderbar, hell

13 und der Garten ist auch da und die Betten sind ein bisschen breiter. Oder Lesen, meine Frau

14 und ich wir lesen unglaublich viel. Und ich freu mich darauf.

$15 \quad[\ldots]$

16 LB: Aber man spielt doch durchaus auch zusammen.

But you are playing with others, aren't you?

17 Burkhard Rosenfeld: Ja, ja klar schon, ist ein Wettbewerb. Und der Clou besteht darin und

18 das gibt es eben in keinem anderen Sport. Im Tennis wenn ich den Ball retourniere, bumm,

19 bumm,weg, Aufschlag 15:0. Das gibt es beim Golf nicht. Im Golf spielt man bis zum Ende,

20 bis der Ball im Loch ist. Und, das ist der Clou, ja, beim Tennis wenn ich gegen einen Gegner

21 habe wie den Dr Kill zum Beispiel, kriege ich keine Schnitte...ich habe nicht schlecht Tennis

22 gespielt und habe sogar mit ihm zusammen da dieses Doppel gewonnen gegen unseren

23 größten Gegner da bei irgend so einem Wettbewerb. Aber wenn mit ihm spiele kriege ich

24 keine Schnitte. Das Gefühl brauch ich nicht. Da spielen Leute die unglaublich gut spielen

25 gegen Leute die eben schlechter spielen und damit, die in Wettbewerb treten können gibt es

26 das Handicap d.h. wenn jemand Handicap null hat, dann, das Handicap ist ne Vorgabe. Dann 
1 hat der keinen vor, muss der eben beim Par vier, mit vier drin sein sonst bekommt der keine

2 zwei Punkte beim Stableford und ich bekomme beim schweren, bei meinem Handicap, beim

3 schweren Par vier zwei Punkte vor. Das heißt der Gute der Profi da, der muss mit vier rein,

4 ich gehe mit sechs rein und wir bekommen beide zwei Punkte. Und das ist schön. Jeder

5 kann mit jedem spielen. Das ist wunderbar. Auch Frauen, Frauen haben einen verkürzten

6 Abschlag, die kriegen halt weniger Länge zugeteilt zum Spielen und man kann es bei jedem

7 Wetter spielen... es ist ein wunderbarere Sport und eine tolle Erziehung für Kinder, tolle

8 Erziehung: Disziplin, Rücksichtnahme, Fairness auch gegen sich selber.

Yes, yes, naturally, it is a competition. And the clou is, and that doesn't exist in any other sports...well, in tennis when I have an opponent such as Dr Kill, for example, I have no chance...That feeling I don't need. There are people who are very good who play against people who are, well, not so good and so that they compete there is the handicap...That is wonderful ...it is a wonderful sport and a great education for children, great education: discipline, consideration for others, fairness also against oneself.

$9 \quad[\ldots]$

10 LB: Und Ihre Frau spielt aber auch?

And your wife also plays?

11 Burkhard Rosenfeld: Die habe ich rumgekriegt, ja.

I convinced her, yes.

12 LB: Und ist ähnlich begeistert jetzt? Oder noch nicht so ganz?

And is she similarly enthusiastic?

13 Burkhard Rosenfeld: Nicht so. Sie hat da mehr Probleme als ich so mit der Kommunikation 14 mit den Frauen. Die Frauen .... Bei den Männern ist es eben sportlich, die schummeln 15 vielleicht ein bisschen mehr die Männer aber sonst...

...For men it is about being sporty, they might cheat a little bit more but besides...

16 LB: Und schimpfen vielleicht auch ein bisschen mehr, da kommt es eher raus. Also ich habe

17 das Gefühl...die Männer sind halt ...

And perhaps they also curse a bit more ...Well, I have the feeling ... men are... 
1 Burkhard Rosenfeld: Die Frauen haben Ehrgeiz und Klamotten, und andiamo.

Women have ambition and clothes and Andiamo.

2 LB: Na gut wenn Sie, Sie gehen ja auch regelmäßig zum Arzt. Demzufolge haben sie 3 durchaus im Kopf, jetzt ja früher nicht?

Ok, when you, you see the doctor regularly. Therefore you think, now yes but previously not?

4 Burkhard Rosenfeld: Immer, also Moment aber in einem bestimmten Alter so ab, würd 5 sagen ab 40, habe ich jedes Jahr einen... Ich habe einen Toparzt mir gesucht und geh da hin

6 und lass mich total durchchecken und dann findet der auch irgendwas, wenn was zu finden 7 ist.

Always, wait a moment, but at a certain age, roughly from when I was 40, I saw every year...I found a top doctor, and I go there, being checked through thoroughly and then he will find something if there is something to find.

$8 \quad[\ldots]$

9 LB: Aber eher etwas was sie psychisch einschränkt oder physisch. Es gibt ja klar es gibt 10 natürlich auch das tatsächliche Worst Case Scenario wo beides eingeschränkt ist aber 11 normalerweise kann man ja sagen ..

But more something which limits you physically or mentally...

12 Burkhard Rosenfeld: Vor allem geistig. Also wenn ich ganz weggetreten wäre es für mich 13 nach meiner Vorstellung schmerzlos.

Especially mentally. Well, if I am mentally entirely gone then in my imagination, it would be totally painless...

14 LB: Also so etwas wie Alzheimer wo man dann...

So something like Alzheimers where you...

15 Burkhard Rosenfeld: Und wenn ich körperlich fit wäre aber blöd würde es mich nicht sehr 16 bewegen.

And if I was physically fit and stupid it wouldn't concern me much. ... 
$1 \quad$ LB: Das heißt körperlich nicht fit aber geistig fit wäre für Sie eher zu verkraften.

That means physically not fit but mentally fit you would find more manageable?

2 Burkhard Rosenfeld: Geistig ist wichtig, ja. Die geistige Fitness...

Mental is important, yes. The mental fitness

3 LB: Aber das würde ja dann auch bedeuten dass Sie sehr viel Hilfe bräuchten. Körperlich,

4 also wenn Sie körperlich eingeschränkt sind, ist das kein Problem für Sie?

But that would then mean that you would need a lot of help... would that not be a problem for you?

5 Burkhard Rosenfeld: Ja, natürlich, das ist alles ein Problem. Mal sehen wie es ist wenn es 6 soweit ist.

Yes, of course, that all is a problem. Let's see how it is when the time comes.

7 LB: Aber grundsätzlich setzen Sie sich damit auseinander oder versuchen Sie eher durch,

8 durch die jährlichen Arztbesuche das einfach und dann hat man noch mehr sozusagen...

But do you generally think about these things or do you try through the yearly visits at the doctors...

9 Burkhard Rosenfeld: Ich tue das was ich tun kann mit Einschränkungen. Also ich

10 verzichten nicht auf Zucker oder Alkohol und rauche nicht und auch keinen scharfen

11 Alkohol. Ich bin auch so vernünftig aber ich mache keinen, ich versuche nicht mich durch

12 irgendwelche Homöopathie oder was weiss ich gesund zu halten sondern vertraue darauf

13 dass oder anders ich bin bereit zu ertragen was dann kommt und damit ich rechtzeitig weiss

14 was kommt und auch noch eingreifen kann gehe ich jedes Jahr zum Arzt.

I do what I can do within limits. So I don't abstain from sugar or alcohol, and I don't smoke or drink spirits. I am also generally sensible, but I don't do, I don't try through some homoeopathy or something to stay healthy, but I trust that, or differently, I am ready to endure what there comes and so that I know what is coming in time and can influence it, I see the doctor every year.

15 LB: Haben Sie generell Angst vor dem Tod?

Are you generally afraid of death? 
No.

2 LB: Weil Sie gläubig sind?

Because you believe in God?

3 Burkhard Rosenfeld: Ich suche nach dem Glauben.

I'm searching for faith.

4 LB: Ohh, das ist aber. Das heißt, haben Sie gesagt Sie sind evangelisch aber sie sind sich

5 nicht so richtig überzeugt von der Existenz Gottes.

...So you are not entirely convinced of the existence of God?

6 Burkhard Rosenfeld: Doch. Ja doch ich bin überzeugt von der Existenz Gottes. Was die

7 Einzelheiten angeht... Bin ich ein zu konkreten Menschen, um richtig gläubig zu sein. Aber

8 ich habe als Grundlage ein gewisses Gottvertrauen.

I am convinced that God exists. With reference to the details...I am a person who is too concrete to really believe. But I have a certain trust in God as a basis.

9 LB: Und demzufolge denken Sie auch dass damit nicht das Ende sondern das da in 10 irgendeiner Form weiter geht.

And therefore you believe that it is not the end but that in some form it will continue.

11 Burkhard Rosenfeld: Ich glaube an, an das ewige Leben und zwar sehr innig, nach dem

12 Glaubensbekenntnis zu sagen. Ich hab einen Geschäftsführer, den ich zu betreuen hatte, mit 13 dem auch gut befreundet war, den ich auch geduzt habe. Der wurde 65, hat ein Jahr vorher

14 von einer Einladung ausgesprochen von einer Abschiedsfeier aus dem Beruf mit 65. Und

15 kurz vor dem Termin hat er abgesagt weil ein Blasenkrebs hatte und da hat er nur noch

16 relativ kurze Zeit gelebt. Mit dem habe ich mich sogar geduzt einer der wenigen, nein es

17 war der war der Einzige, es war der Einzige in meinem Berufsleben jedenfalls in der

18 Induszeit. Bei dem war ich am Bett im Krankenhaus am Tag bevor er endlich erlöst wurden.

19 Da hab ich gesagt Wolfgang, was meinst du was danach ist? Nichts. Nichts. Nichts...ein

20 richtig toller Mensch an sich aber ... 
I believe in, in eternal life and that very deeply...I was in the hospital at his bed one day before he finally passed. Then I said, Wolfgang, what do you think, is there after? Nothing. Nothing.... a really great person but...

$1 \quad$ LB: Was ja auch ein Glaube ist.

Which also is a belief.

2 Burkhard Rosenfeld: Ja, ok, aber es hat mich ein bisschen traurig gemacht. Und bei mir ist

3 es das Gegenteil, ich glaube dass da nicht nichts ist.

...I believe that there is not nothing.

$4 \quad[\ldots]$

$5 \quad$ LB: Oh, das ist ja nicht so das typische Urlaubsziel.

That is not the typical holiday destination.

6 Burkhard Rosenfeld: Absolut nicht. Aber wir haben alles Mögliche überlegt. Meine Frau 7 ist da noch ein bisschen neugieriger als ich. Wir haben uns auf Armenien geeinigt.

8 Armenisch ist ja in aller Munde. Ich hatte eine armenische Studienfreund, ein Armene, der 9 ist dann im Iran aufgewachsen, dann sind die geflüchtet und in England und plötzlich war

10 der in München und hat da studiert ohne das zu dürfen eigentlich. Da haben wir uns als ich

11 fertig war uns aus den Augen verloren und sind uns wieder begegnet vor 10 Jahren. Da hat

12 er sich gemeldet als Deutsch Professor aus der Schweiz

...My wife is even a little more curious than me....

$13[\ldots]$

14 LB: Es ist so interessant mit ihnen und ich habe diese ganzen doofen Fragen hier eigentlich

15 gar nicht so... Da wir nämlich gerade bei dem Thema sind können wir mal machen wir das

16 jetzt mal. Weil Teil meiner Arbeit besteht darin dass ich Filme analysiere. Das heißt ich

17 beschäftige mich mit Mediendarstellung von Alter eher untypische Mediendarstellung von

18 Alter nämlich in Actionfilmen. Aber grundsätzlich interessiert mich natürlich wie Sie das

19 wahrnehmen. Wie Alter in den Medien dargestellt wird. Und ich habe ja ein Test Interview

20 mit meinem Vater gemacht und habe dabei festgestellt dass es schwierig ist das so aus der

21 Schublade zu holen. Deswegen habe ich ein paar Bilder mitgebracht die relativ typisch sind

22 für die Darstellung von Alter im Augenblick. Vielleicht fällt Ihnen auch selber ein wo sie 
1 schon mal irgendwie Alter gesehen haben, was zu sagen gibt, es gibt quasi eine zweiseitige

2 Darstellung. Zum einen die sehr positive Darstellung wie Sport oder Gelassenheit. Und dann

3 gibt es diese sehr negative die jetzt nicht mehr so ganz in ist aber... Aber fühlen Sie sich

4 wenn sie das sehen, fühlen sich da in irgendeiner Form repräsentiert ist das etwas wo sie sich

$5 \quad$ wiederfinden können?

...But do you feel represented in some way (by the visuals), is there something which you can identify with?

6 Burkhard Rosenfeld: (sehr lange Pause) Das, das und das (Fotos von aktivem Alter)

(Long break). That, that and that (pictures of active ageing).

7 LB: OK. Das ist das für sie wo sie sagen das passt. Aber so grundsätzlich wenn Sie sich

8 überlegen. Man sieht ja schon mal was ich im Fernsehen, Filme wie auch immer. Haben Sie

9 das Gefühl dass sie das in irgendeiner Form repräsentiert, das das ihr Leben

10 widerspiegelt.(keine Antwort) Ja, ich weiß nicht. Schauen Sie Filme, gucken Sie Fernsehen?

...But generally...one sometimes sees something on TV, films whatever. Do you feel that this represents you in any way? (No answer) Well, I don't know. Do you watch films or do you watch TV?

11 Burkhard Rosenfeld: Wenig, eher nicht, Nachrichten Sport. Aber keine ...

Little, not really. News, sports. But not...

12 LB: Ja.

Yes.

13 Burkhard Rosenfeld: Aber Filme, meine Frau hat, meine Frau könnte jede Woche ins Kino

14 und ich gehe mit ihr regelmässig, ich glaube einmal im Monat, dann sehen wir Filme, jetzt 15 muss ich überlegen was wir da gesehen haben. Es gibt schon ....

But films, my wife, my wife, could go to the cinema every week and I join her regularly, I believe once a month...

16 LB: Ja.

Yes. 
1 Burkhard Rosenfeld: Es gibt schon. Mir fällt jetzt konkret keiner ein.

It exists. I can't think of one right now.

2 LB: Es gibt dieses Hotel Marigold mit den ganzen britischen Schauspielern. Aber zum

3 Beispiel was mich interessiert, ich nehme mal an dass sie diese Filme nicht gucken, aber es

4 gibt sehr viele Actionfilme mittlerweile mit doch relativ alten Männer. Sylvester Stallone

5 spielt nach wie vor in Filmen mit, Arnold Schwarzenegger.

...there are many action films with rather old men. Sylvester Stallone still acts in films, Arnold Schwarzenegger...

6 Burkhard Rosenfeld: Das beeindruckt mich nicht, nein.

That doesn't impress me, no.

7 LB: Nein.

No.

8 Burkhard Rosenfeld: Das beeindruckt mich nicht. Mich beeindruckt eher wenn jemand 9 aufgrund seines Alters ... Doch da gibt es doch den einen Film der den deutschen Film, der 10 auch prämiert wurde. Wie hieß der gleich? Da besucht ein Vater seine Tochter, die irgendwie 11 im PR Management ist, irgendwo in Ungarn oder irgendwo ....

That doesn't impress me. I am more impressed when somebody because of his age...

12 LB: Ich habe auch so eine vage Erinnerung aber es kommt mir kein Titel.

There is a vague memory, but I can't remember the title.

13 Burkhard Rosenfeld: Dieser Film der also auch im internationalen Wettbewerb stand und 14 dort auch einen Preis bekommen hat. Da hat der Vater sich sehr klug verhalten, es war viel 15 komisch. Eigentlich hat der Vater sie gestört, der hat da viel Quatsch gemacht.. sich sehr 16 weise verhalten, das, das imponiert mir, das gefällt mir. ...[he] behaved very wisely, that, that impresses me, that I like.

17 LB. Das heißt wir sind wieder bei Weisheit, Gelassenheit und das ist so was für sie Alter 18 darstellt, männliches Alter. Denken Sie dass es für Frauen schwieriger ist alt zu werden als 19 Männer? 
That means we are back with wisdom, placidity, and that is what represents older age, older male age. Do you think that it is more difficult for women than for men to grow older?

1 Burkhard Rosenfeld: Ja.

Yes.

2 LB: Warum?

Why?

3 Burkhard Rosenfeld: Ich glaube dass Frauen im Schnitt, leiden Frauen mehr an den

4 körperlichen Zeichen des Alters, Falten, Brille, was weiss ich.

I think on average women suffer more by the physical signs of ageing, wrinkles, glasses, whatever.

5 LB: Aber man könnte ja auch sagen weil ich meine Männlichkeit ja durchaus auch nur mit

6 Kraft, mit Dominanz, Kontrolle was ja dann, das ist ja sozusagen das Thema meiner

7 Doktorarbeit, was im Alter doch zunehmend zumindest geringer wird. Das ist aber nicht

8 etwas was ihnen zu schaffen im Hinblick auf Identität, Vorstellung von Männlichkeit?

But you could say because masculinity is linked to strength, control... which more and more decreases with older age. That is but not something which worries you with reference to identity, the conception of masculinity?

9 Burkhard Rosenfeld: Nein, macht mir nicht zu schaffen. Es gibt Dinge mit denen ich mich

10 beschäftigen muss. Mit denen ich aber ganz gut fertig werde weil ich einfach eine gewisse

11 Demut habe, dass ich einfach Dinge akzeptieren möchte und nicht jammere oder versuche

12 sie unter den Tisch zu kehren. Das ist so und jetzt muss ich damit zurechtkommen. Ich hatte

13 ein Herzoperationen 2011, da war meine Aortenklappe zu. Da kam der Operateur kaum noch

14 mit der Nadel durch als sie dann mich aufgesäbelt haben. Ich bin da reingegangen... Es war

15 ein Prozess von Jahr zu Jahr wurde die Öffnung kleiner, Verkalkung und irgendwann war es

16 nicht mehr zu vermeiden. Da musste ich es machen. Ich habe in dem Jahr... Ich habe den

17 Termin hinausgezögert bis zum letzten Moment, es war wahrscheinlich der allerletzte

18 Moment. Einerseits wegen meiner Indusaufgabe und andererseits ... nein, ich habe wegen

19 der Indusaufgabe das hinausgezögert. Ich habe in dem Jahr eine Tour gemacht in meine

20 Heimat Ostpreußen. Da sind wir nach Litauen geflogen und sind mit dem Fahrrad bis nach

21 Danzig gefahren in Abständen und ich konnte nicht mal so einen kleinen Berg mit dem 
1 Fahrrad hochfahren. Die Leute haben immer dann gewartet und meine Frau war schon ganz

2 nervös. So stark war ich eingeschränkt. Ich war so froh als wir an der Weitzel standen. Da

3 gibt es ein glückliches Foto dass ich das überlebt habe. Ich habe natürlich nicht dran gedachte,

4 dass ich es nicht überleben könnte aber ich hab gemerkt wie das schlimm ist. Das hat mich

5 schon geschlaucht. Aber ich habe es akzeptiert. Ich wusste woran es lag und bin dann in die

6 Operation gegangen und ich fühlte mich fit, fühlte mich in sehr guten Händen und habe an

7 sich nicht daran gezweifelt, dass ich da wieder sehr gut rauskomme.

No, that doesn't worry me. There are things I have to think about. Which I am, however, handling quite well since I have a certain humbleness that I want to accept things and don't complain or don't want to kick into the long grass. That is the way it is, and now I have to handle it...

8

LB: Sehr ungewöhnlich. Das habe ich auch noch nie erlebt, dass jemand gefeiert hat nur weil er feiern möchte. Das ist schön, das ist sehr, sehr schön. Sie sind ziemlich viel ja nicht nur jetzt sondern auch vorher schon gereist das heißt sie haben ja ein bisschen Erfahrung mit unterschiedlichen Kulturen. Denken Sie ... wie würden Sie denn die Einstellung zum Alter in unserer Gesellschaft beschreiben? Gibt es Unterschiede zu anderen Kulturen?

...How would you describe the attitude towards ageing in our society? And are there differences to other cultures?

Burkhard Rosenfeld: Ja also das was mir spontan einfällt. Europa und Japan. In Japan ist die Seniorität eben eine Stufe im Leben, ist die höchste Stufe im Leben. Also ein alter Mensch ist dort, spielt dort die dominante Rolle. Bei uns in Europa ist das nicht so. In Europa git es das Altenteil und die alten Rentenempfänger, die den Jungen das Geld wegnehmen oder, was weiss ich, oder die Alten die an ihrem Job klemmen, kleben die nicht loslassen wollen. Da ist... zwischen diesen beiden Kulturen ist für mich der größte Unterschied.

...In Japan seniority is simply one level in life, the highest level. So an older person there plays a dominant role. With us in Europe that is not the case. In Europe, there is retirement and the old receivers of a pension who take away money from the young or whatever or the old ones that are glued to their jobs, who don't want to let go. That is... for me the biggest difference between the two cultures.

LB: Aber woher kommt das, denken Sie, diese fehlende Wertschätzung von Alter in Europa und ich würde mal vermuten auch den USA. 
But where do you think this lack of appreciation in Europe and I would also guess the US comes from?

1 Burkhard Rosenfeld: Ich möchte ein Land auswählen: Italien.

I want to choose a country: Italy.

$2 \quad$ LB: Auch Spanien oder auch Malta...

Also Spain or even Malta...

3 Burkhard Rosenfeld: Ja, ja, ja, ja also in Mitteleuropa, in Deutschland sind die Familien

4 durch die Kriege, die Fluchten und so weiter, durch das Wesen der Menschen, Ehrgeiz,

5 Arbeit und so weiter auseinandergetrieben worden und das hat auch einen Einfluss auf die

6 Werte der Gesellschaft. In den Mittelmeerländern spielt die Familie immer noch eine viel

7 größere Rolle, ist auch meistens... Erstens gab es diese kriegerischen Auseinandersetzungen

8 wie in Mitteleuropa nicht. Zweitens, ist es die wirtschaftliche Situation, die komplett andere

9 wirtschaftliche Situation in diesen Ländern, die dazu führt das der Herr Asunto nach wie

10 vor zu seiner Mama nach Calabrien fährt. Weil die Familien oftmals, mehrere Generationen

11 im gleichen Haus wohnen, auch die die Eignungsverhälnisse ist eine Situation bei uns in

12 Deutschland gibt es an sich in Mittelmeerländern weniger, da ist das Leben im Eigentum,

13 sei es noch so einfach, normaler als hier.

...well, in the middle of Europe, in Germany families have been driven apart as a result of wars, abscondences and so on, as a result of the human character, ambition, employment and so on and that has an influence on the values of a society. In Mediterranean countries this doesn't exist. Firstly, they didn't have war-like conflicts as in the middle of Europe. Secondly, it's the economic situation, the totally different economic situation in these countries that has led to the situation that Mr. Asunto still travels to his mother in Calabria. Because frequently the family, several generations live in the same house...

14 LB: Ja trotzdem ich kann es nicht. Ich kann es immer noch nicht so richtig nachvollziehen

15 warum Alter grundsätzlich in europäischen und nordamerikanischen Ländern eher ein 16 negatives, einen negativen Beigeschmack hat.

Yes, nevertheless, I can‘t. I can’t understand why older age in European and North-American countries is rather linked to negative attributes. 
1 Burkhard Rosenfeld: Weil die Leistungsfähigkeit abnimmt, weil die Jungen sehr früh

2 getrimmt werden nach oben und dann wenn man dann da ist und da ist da noch so ein Alter,

3 dann stört der. Das ist die Leistungsgesellschaft.

Since efficiency declines, since the young very early on are trimmed to go to the top and then when you are there and then there is an old one, he disturbs. That is a meritocracy.

4 LB: Und das wiederum ist halt schon etwas durchaus von Amerika zum Teil

5 rübergeschwappt ist. Wir haben diesen Mix auch aus Sozialstaat und eben sehr krasser

6 Wettbewerbsgesellschaft, neoliberaler Gesellschaft. Wir sind fast bei der letzten Frage,

7 schließt sich an. Wenn sie mit jüngeren Menschen zu tun haben, haben Sie den Eindruck

8 dass sich jüngere Menschen Ihnen gegenüber anders verhalten als Gleichaltrigen oder

9 Jüngeren?

...When you are dealing with younger people do you have the impression that the behave differently to you than to people their age or younger?

10 Burkhard Rosenfeld: Ja.

Yes.

$11 \quad$ LB: Inwiefern und ist das eher positiv. Ist das positiv für sie oder eher negativ?

In what way and is that more positive. Is that more positive or more negative?

12 Burkhard Rosenfeld: Jüngere Menschen befinden sich in einer ganz andere

13 Lebenssituation, haben ganz andere Ziele und Probleme als ältere Menschen. Ältere

14 Menschen haben die Neigung mit ihrer Erfahrung zu protzen und Recht zu haben, Recht

15 haben zu wollen. Und das ergibt, und das ergibt einfach Konflikte, manche offen, manche

16 nur verborgen. Also, wenn ich jetzt mich nehme mit jungen Leuten, ja, ich kann es schlecht

17 einschätzen. Es ist wahrscheinlich so... Die Dominanz die man selbst repräsentiert auch

18 wenn man sich, wenn man das nicht aktiv macht, schränkt die Freiheit und die Leichtigkeit

19 ein.

Younger people are in a completely different life situation; they have totally different aims and problems than older people. Older people have the tendency to brag with their experience and always think they are right; they want to be right. And that leads to conflicts, some open and some only hidden. Well, if I'll take myself, well, I can't really judge. It is 
probably like this... The dominance that one represents, even if one doesn't do this actively, limits the freedom and ease of others.

$1 \quad$ LB: Das heißt man ist nicht auf einer Ebene und kann dann ...

That means you are not on the same level and can then ...

2 Burkhard Rosenfeld: Man möchte, man möchte gerne auf eine Ebene, ist aber nicht auf 3 einer Ebene.

You want to be on the same level, but you are not on the same level.

4 LB: Haben Sie den Eindruck dass Sie jungen Menschen gegenüber in bestimmten Bereichen 5 im Nachteil sind.

Do you think that you are at a disadvantage in comparison to younger people in certain areas?

6 Burkhard Rosenfeld: Ja klar. Ich... ich habe nicht diese Unbekümmertheit. Ich lebe im, in 7 einer ganz bewussten Endlichkeit. Als ich jung war gab es das, den Begriff für mich nicht 8 da war einfach das Leben.

Yes, of course. I ... I don't have this carefreeness. I live in a conscious finality. When I was young that, the term, did not exist for me - there simply was life.

$9 \quad[\ldots]$

10 Burkhard Rosenfeld: Aber ich bewundere die jungen Leute wie das annehmen und ich

11 lach mich immer kaputt wenn ich irgendwo fahre und da sehe überall die Kinder rumlaufen

12 - und die Erwachsenen auch.

But I admire the young people how they accept this und I have to laugh out loud when I drive somewhere and I see children and adults running around. 


\section{H Interview \& Translated Extracts Prof. Dr Günter Schotten}

1 LB: So, okay, dann fangen wir direkt mal mit der sozusagen entscheidenden Frage an.

2 Nämlich was bedeutet Männlichkeit für Sie?

...What does masculinity mean to you?

3 Prof. Dr Günther Schotten: Also Männlichkeit bedeutet für mich eine... Der Versuch

4 stringent zu denken und auch eine gewisse körperliche Anstrengung und ein Vernunftbezug,

5 stark vernunftbezogenes Leben.

Well, masculinity means to me a...the attempt to think stringently ${ }^{24}$ and also a certain amount of physical effort and a strong reference to rationality, a highly rational life.

6 LB: Und das ist schon entgegengesetzt Weiblichkeit?

And that is in contrast to femininity?

7 Prof. Dr Günther Schotten: In einer gewissen Form ja.

To a certain degree, yes.

$8 \quad[\ldots]$

9 LB: Das heißt rational, irrational oder emotionsbezogen?

10 That means rational, irrational or with reference to emotions?

11 Prof. Dr Günther Schotten: Ja, Emotionen nicht so stark. Ja, stark vernunftbezogen. Stark

12 auf... Auch leistungsbezogen. Das ist, glaube ich, der allerwichtigste Unterschied, dass die

13 Leistungsbezogenheit. ... Die scheint ein männliches Gen zu haben.

Yes, emotions not so much. Yes, rational. Strongly focussed on... also performanceoriented. That is, I believe, the most important difference, the performance orientation ...This seems to have a male gene.

$14 \quad[\ldots]$

15 LB: Denken Sie das dieses Bild was Sie von Männlichkeit haben, was mit Ihrem

16 Aufwachsen zu tun hat? Mit ihrer Familie? In irgendeiner Form?

\footnotetext{
${ }^{24}$ The German word 'stringent' is linked predominately to notions of coherence, logical thinking and rationality and not as in English to being strict or forceful
} 
Do you think that your conception of masculinity is somehow linked to your upbringing? Your family? In some way?

1 Prof. Dr Günther Schotten: In einer gewissen Form schon, ja. Ich meine man ist ja schon 2 geprägt durch das Elternhaus, Großeltern, durch was man so erlebt hat. Und das glaube ich 3 schon. Auch durch ein traditionelles Rollenbild. Das war ja viel stärker als das heute noch

4 nachvollziehbar ist.

I think that one is somehow influenced by one's parents, grandparents, by what one experiences. I believe that, also, by the traditional role model. That was much stronger than one can understand today.

5 LB: Das heißt, Ihre Eltern waren auch zusammen ihr Leben lang?

That means your parents were together their whole life?

6 Prof. Dr Günther Schotten: Genau, sie sind ihr Leben lang zusammengeblieben. Und mein

7 Vater hat gearbeitet. Der war auch Notar. Und meine Mutter hat sich um die Kinder

8 gekümmert. Sie war sehr außerhäußig, hat auch Politik gemacht, war im Stadtrat, im Kreisrat

9 und so etwas, aber im Endeffekt war diese Rollenverteilung vollkommen da.

Yes, exactly my parents stayed together all their lives. And my father worked. He was a notary, too. And my mother took care of the children. [She] was very extramural, was involved in politics, was in the city council, in the unitary council but in the end, the role division was absolutely there.

$10 \quad[\ldots]$

$11 \quad$ LB: Nein? Warum denn nicht?

No? Why not?

12 Prof. Dr Günther Schotten: Ich bin nicht diplomatisch. Nein überhaupt nicht.

I'm not a diplomatic type...I am not diplomatic. No, not at all.

13 LB: Keine politischen Ränkespiele?

No political intrigues?

14 Prof. Dr Günther Schotten: Nein, kein diplomatischer Typ. Ich bin völlig undiplomatisch.

15 Völlig undiplomatisch.

No, not a diplomatic type. I am totally undiplomatic. Totally undiplomatic. 
2 LB: Und wieso gerade Bielefeld? Was...

And why Bielefeld? What...

3 Prof. Dr Günther Schotten: Bielefeld, das hat sich ergeben... Bielefeld hat eine einstufige

4 Juristenausbildung gehabt. Da hat man versucht Theorie und Praxis direkt miteinander zu 5 verbinden. Es hat drei Universitäten gegeben. Das waren so Reformuniversitäten. Das war

6 Konstanz, Bielefeld und Bremen. Bremen war rein sozialistisch aufgezogen und ist ganz 7 schnell gescheitert. Konstanz und Bielefeld hatten großen Erfolg. Die Leute haben früher 8 Examen gemacht, besser Examen gemacht, waren viel besser ausgebildet. Problem ist nur,

9 man konnte schlecht wechseln, weil man im System war und es war sehr arbeitsintensiv,

10 sehr kostenintensiv. Man brauchte viele Professoren. Mehr als sonst und deswegen ist das 11 auch finanziell irgendwann gescheitert. Dann hat man das aufgegeben und da gab es in 12 Bielefeld eben eine praxisbezogene Ausbildung. Und da wurden sogenannte 13 kautelarjuristische Hausarbeiten ausgegeben. Also, das sind Hausarbeiten über 14 Rechtsgestaltung. Im juristischen Prüfverfahren wird immer nur geprüft, Fälle die 15 abgeschlossen sind. Wie man das dann juristisch subsumiert und Rechtsgestaltung ist ganz 16 anders. Da macht man Rechtsgestaltung im hohen Maße jedenfalls. Der muss eigentlich 17 vorweg denken, was alles passieren könnte. Als Beispiel, zum Beispiel unsere Regierung hat 18 nicht vorweg gedacht, wenn sie eine Europäische Vereinigung machen und einfach 28 19 Staaten hineinnimmt und keine Regelungen treffen, wenn einer sich völlig falsch verhält. 20 Wenn ich das im Gesellschaftsvertrag mache, sagt mir jeder Richter nachher: „Ja Herr Notar, 21 warum haben Sie daran nicht gedacht?" Das ist also Rechtsgestaltung und die ist schwierig. 22 Die ist auch schwierig zu bewerten. Da gab es also einen Ausschuss. Da waren viele 23 Bielefelder Professoren drin und ich war da als Notar drin. Und da haben die... Sind mit mir 24 gut zurechtgekommen und haben mich gefragt, ob ich einen Lehrauftrag übernehmen wollte. 25 Das war 1987,88 da rum. Und dann war das richtig gut. Ich hatte auch viel Erfolg. Also ein ganz bekannter Professor, der später Rektor an der Heidelberger Universität geworden ist, der ist mal am Hörsaal vorbeigegangen und da hat seine Assistenz zu ihm gesagt: „, Wenn Sie mal einen vollen Hörsaal sehen wollen, müssen Sie da rein gehen. “War total 200 Studenten. Das ist schon eine ganze Menge. Am Schluss waren es dann aber auch nachher weniger. Es war ganz komisch. Es hatte dann sich auch verschoben. Es war also...

And then that was really good. I also had a lot of success. A really well-known professor... once passed by the lecture hall and then his assistant said: 'If you want to see a full lecture hall, then have a look in here.' There were in total 200 students. That is quite a lot. ... 
2 LB: War das denn wichtig für Sie, diese...?

Was that important for you, this...?

3 Prof. Dr Günther Schotten: Ja, das ist das Männlichkeitsproblem. Leistungsbezogen und 4 mit ganz großer Systematik.

Yes, that is the problem of masculinity. Performance orientation with a strongly systematic approach.

$5 \quad[\ldots]$

6 LB: Das ist ja erstaunlich.

That is astonishing.

7 Prof. Dr Günther Schotten: Ja, das hat mir richtig Spaß gemacht. Da habe ich eigentlich 8 zum ersten Mal auch festgestellt, dass ich eine pädagogische Fähigkeiten hatte. Und habe

9 da auch guten Erfolg gehabt. Habe das über Jahre gemacht und dann ging das eben später 10 ganz schnell in diese Hochschule nach...

Yes, I really enjoyed that. That was the first time that I noticed that I had pedagogical abilities. And that had sound success...

$11[\ldots]$

12 LB: Das ist natürlich schon in jungen Jahren durchaus etwas was einen prägt. Was haben

13 Sie denn... Also wir haben jetzt ein bisschen darüber gesprochen im Bereich Universität, was

14 Ihnen da Freude gemacht hat. Was hat Ihnen denn als Notar Freude gemacht? ...What did you enjoy as a notary?

Prof. Dr Günther Schotten: Als Notar hat mir Freude gemacht, dass man als Notar ganz

16 viel mit Menschen zu tun hat, die mit entscheidenden Problemen zu einem kommen und die 17 nicht so streitbezogen sind. Und der Notarberuf hat ja einen, das sieht Ihr Vater anders, das 18 weiß ich, aber, der sieht das so wie ich, glaub ich jedenfalls. Aber der Notarberuf hat ja einen eigenartigen Ruf. Langweilig, man liest immer nur vor. Das ist ja völlig verkehrt. Man hat mit den zentralen Problemen des Menschen zu tun. Mit Erbrecht, mit Familienrecht, mit Erb-

21 Auseinandersetzungen, mit Gesellschaftsrecht. So jetzt oder auch jetzt bei der Indus mit 22 Unternehmenskaufverträgen. Und man hat wirklich mit den elementaren Bezügen des 23 Menschen zu tun. Ob er zum Beispiel einen Ehevertrag beurkundet. Wie die Rollen verteilt 24 werden sollen. Wie es finanziell ist. Das man auch versucht auch zu gestalten. Das man... 
1 Das nicht jemand über den Tisch gezogen wird. Also im Familienrecht, im Erbrecht, im

2 Gesellschaftsrecht, bei Gründung von Unternehmen und so etwas. Dann kommen

3 Kaufverträge dazu, die relativ schnell abgewickelt sind. Die auch das Geld bringen. Aber

4 die laufen oder ferner liefen. Das andere ist das Entscheidende, wenn man denn dafür ein

5 Interesse hat. Und deswegen...ich war also im Notarberuf sehr, sehr gerne. Ist natürlich

6 völlig im Hintergrund und der Notarberuf, wenn man da erfolgreich arbeiten will, das merkt

7 ja keiner, sage ich jetzt mal oder Leute die vergleichen können. Denn wenn der Notar Fehler

8 macht, dann kommen Prozesse. Wenn Sie nicht verklagt werden, haben Sie im Prinzip nichts

9 verkehrt gemacht. Merkt nur keiner. Es wird bemerkt, wenn nur einer pausenlos verklagt

10 wird. Das ist also so.

What I enjoyed in being a notary, that as a notary you have a lot to do with people who come to you with crucial problems ... You deal with the central problems of people...You really have to do with the essential references of human beings. ...

$11 \quad[\ldots]$

12 LB: Und wichtige Dinge.

And important things.

13 Prof. Dr Günther Schotten: Wichtige Dinge auch. Und ganz wichtig war natürlich auch,

14 dass die Leute Vertrauen hatten und das habe ich glaube ich im relativ hohen Maße auch

15 bekommen.

And of utmost importance was of course also that the people had trust in you and I believe I received that to a relatively high degree.

$16 \quad[\ldots]$

17 LB: Sie haben es also nicht bereut.

So you never regretted anything?

18 Prof. Dr Günther Schotten: Nein, überhaupt nicht. Für mich war die Berufswahl Notar

19 absolut die richtige. Absolut die Richtige.

No, not at all. For me to become a notary was absolutely right. Absolutely right.

$20 \quad[\ldots]$

21 LB: Hatte im Rückblick ihre berufliche Position einen Einfluss darauf wie Sie sich als Mann

22 definiert haben? 
With hindsight, has your professional position had an influence on how you defined yourself as a man?

1 Prof. Dr Günther Schotten: Ja, im ganz hohen Maße. Im ganz hohen Maße würde ich sagen, weil ich glaube, dass ich den richtigen Beruf für mich gewählt habe. Dass das ein

3 Beruf war in dem man sehr intensiv, ruhig, sachlich arbeiten kann. Auch ausgewogen und

4 mit wesentlichen Problemen und das hat, glaube ich, mein Leben schon geprägt.

Yes, to a very high degree. To a very high degree, I would say, since I believe I have chosen the right profession for me. That, that was a job, in which you can work very intensely calmly, rationally. Also balanced and with crucial problems and that, I believe, influenced my life.

$5 \quad[\ldots]$

6 LB: Und hat das einen Einfluss auf Sie? Also wie die Leute dann reagieren?

And that has an influence on you? Well, how people then react?

7 Prof. Dr Günther Schotten: Ja, ja, ja das hat schon einen Einfluss auf mich. Das ist 8 selbstbewusstseinsstabilisierend.

Yes, it does have an influence on me. That is was stabilising for the self-confidence.

9 LB: Ja (lacht). Sie haben vorhin schonmal so angedeutet, wie hat sich denn ihr Privatleben

10 während Ihrer Berufstätigkeit gestaltet?

...how was your private life structured during your career?

11 Prof. Dr Günther Schotten: Mein Privatleben hat sich also so gestaltet, dass ich zu viel

12 gearbeitet habe. Die Kinder... Wir haben zwei Kinder. Eine Tochter und einen Sohn und

13 meine Frau sagt auch immer sie sei Alleinerziehende gewesen. Da ist sicher auch etwas

14 Wahres dran. Ich habe viel, viel gearbeitet. Aber bin auch... Wir sind immer mit den Kindern

15 in den Urlaub gefahren. Wir haben interessante Reisen gemacht. Wir sind nach Israel

16 gefahren, wir sind nach Amerika gefahren, nach England, nach Spanien und so etwas, aber

17 immer mit den Kindern. Da bin ich ganz...Im Urlaub bin ich auch ganz bei der Familie

18 gewesen, aber sonst habe ich zu viel gearbeitet. Gar kein Zweifel.

My private life was structured so that I worked too much...my wife always says that she was a single mother. There is certainly some truth in this. I worked a lot, a lot. But I also...We always went on holidays together with the children, but besides that, I worked too much, no doubt about it. 
Also, at weekends?

2 Prof. Dr Günther Schotten: Auch am Wochenende. Jaja und viele Abende, jaja. Oft. Und

3 dann bin ich auch viel unterwegs gewesen zu Vorträgen und sonst etwas. Das ist (seufzt),

4 wenn man es so Revue passieren lässt, ist vielleicht eine falsche Wertung. Dass man da zu

5 viel Wert daraufgelegt hat.

Also at weekends. Yes, yes, and many evenings, yes, yes. Frequently. And then I was away a lot for public lectures or something. That has (sighs), when thinking back, may have been the wrong evaluation. That one put too much value in it

6 LB: Das heißt Sie hätten sich... Im Rückblick könnten Sie sich vorstellen, dass es schön 7 gewesen wäre, wenn Sie mehr Zeit...

With hindsight, can you imagine that it would have been nice to have some more time ...

8 Prof. Dr Günther Schotten: Ja, wenn man es anders gemacht hätte. Ja, aber da kommt 9 jetzt, sage ich mal, das männliche Gen, Leistungsbewusstsein. Das man auch eine 10 Anerkennung von außen in einer gewissen Form auch, ich will nicht sagen extrem sucht, 11 aber wenn man sie bekommt auch zu schätzen weiß. Und das führt dann sicherlich auch zu 12 gewissen Problemen. Die wir aber immer gelöst haben. So ist es nicht.

Yes, there is, let's say, the male gene, performance orientation. That one I don't want to say explicitly seeks but appreciates if one receives social recognition from the outside...

$13[\ldots]$

14 LB: Ja, aber der Unterschied ist eben, dass Sie sich zusammengerauft haben und dass es 15 eben bei vielen anderen dann einfach nicht mehr klappt. Also und ich... Das ist leider ein 16 anderes Thema. Das hat mich früher immer sehr interessiert, warum das in manchen Ehen 17 klappt und in manchen nicht. Hat denn... Aber das können wir ja schonmal sozusagen vorab 18 machen jetzt - Hat Ihre Frau auch gearbeitet?

... Did your wife also work?

19 Prof. Dr Günther Schotten: Ja. Die war Rechtsanwältin. Die war Juristen. Ja, die war 20 Rechtsanwältin und die hat eine sehr kleine Praxis gehabt, aber eine sehr erlesene Praxis. 21 Und ich habe ihr auch sicherlich viel geholfen. Ich habe sie animiert dazu etwas zu machen. 22 Sie hat zeitweise bei mir etwas gearbeitet. Das hat aber nicht so geklappt. Dann habe ich 23 also... Wenn sie mir Entwürfe gemacht hat, dann habe ich das kontrolliert und verbessert. 
1 Das geht nicht. Man kann... Das sollte man nicht tun. Haben wir auch sehr schnell erkannt,

2 dass das nicht geht. Und dann habe ich ihr geraten, mach eine kleine Anwaltspraxis auf.

3 Mach Scheidungssachen, Erbrecht, Gesellschaftsrecht, Jugendstrafrecht, hat sie gemacht

4 und auch mit gutem Erfolg, aber klein. Sehr klein und hat das immer zurückgestellt. Die

5 Kinder sind zeitlich immer vorgegangen und wenn also irgendwas war... Deswegen hat das

6 bei ihr keine so große Rolle gespielt.

...but small. Very small and it always took backstage. The children had time-wise always preference and when there was something ... That is why it ${ }^{25}$ did not play such a big role for her.

$7 \quad[\ldots]$

8 Prof. Dr Günther Schotten: So viel hat meine Frau auch gearbeitet, aber wie gesagt, völlig

9 im Hintergrund und ganz wenig. Im Verhältnis zu mir natürlich ganz wenig.

My wife worked much but, as said, always totally in the background and very little. In comparison to me, naturally very little.

10 LB: Und sich um Ihre Kinder dann gekümmert?

And then took care of your children?

11 Prof. Dr Günther Schotten: Genau das war immer Vorrang. Was ja auch richtig war.

12 Deswegen hat sie sich auch als alleinerziehend bezeichnet.

Exactly, that always had preference. Which was appropriate ${ }^{26}$. That is why she called herself a single mother.

$13[\ldots]$

14 LB: Ja aber so richtig?

Yes, but really?

15 Prof. Dr Günther Schotten: Richtig seit... Gut jetzt habe ich den Staudinger nochmal neu 16 geschrieben. Mein IPR-Buch das habe ich schon nicht mehr gemacht. Dritte Auflage, die ist 17 jetzt von anderen herausgekommen vor ein paar Monaten. Da habe ich 2007 das letzte Mal 18 so ein relativ 700-Seiten dickes Buch geschrieben. Dann von 2008 bis 2012 bei der Indus, 19 diese Anwaltstätigkeit und jetzt letztes Jahr ist der Staudinger gekommen und der kommt in

\footnotetext{
${ }^{25}$ the job

${ }^{26}$ The interviewee used the word, richtig' which literally translated would be 'correct' but within this context he referred to the appropriateness of behaviour in his eyes and not some objective standard of correctness. I therefore chose the word appropriate instead of correct
} 
1 fünf Jahren.- Alle fünf Jahre kommt der neu. Also insofern seit einem Jahr tue ich zurzeit

2 juristisch gar nichts.

As such, I haven't done any legal work for a year.

3 LB: Na gut. Dann sagen wir mal in dem Moment wo Sie aus sozusagen Ihrer Haupttätigkeit

4 ausgestiegen sind, also mit 66, wie war das denn für Sie? Also der Übergang?

...when you stopped with 66 , how was that for you? Well, the transition?

5 Prof. Dr Günther Schotten: Der Übergang war für mich... Mein Sohn ist ja Psychiater

6 und er hat mir gesagt, ich hätte das erstaunlich gut geschafft. Ich habe das erstaunlich gut

7 geschafft. Ich hatte natürlich wohl noch... Ich habe erstmal eine ganze Reihe Hobbies, sage

8 ich jetzt mal. Das Laufen. Dann Philosophie. Ich mache relativ viel Philosophie und das war

9 dieser Professor für Philosophie, der da eben angerufen hat. Kommen wir vielleicht gleich

10 noch drauf. Und habe dann wohl an dem IPR-Buch nochmal gearbeitet. Die zweite Auflage.

11 Die erste Auflage habe ich 1995 geschrieben und die zweite Auflage ist dann 2007

12 rausgekommen. Da habe ich zwei Jahre lang doch auch 30, 40 Stunden die Woche gearbeitet.

13 Das ist viel Arbeit gewesen. Und habe dann hin und wieder auch einen Aufsatz... Es gibt so

14 Themen, die ganz wichtig sind in diesem Bereich und wenn es dann... Da gibt es einen

15 Professor Otte, den ich besonders schätze. Der mich auch gefragt hat, ob ich im Staudinger

16 da mitschreiben würde und der hat eine These vertreten, die ich für falsch halte und die also

17 von größerem pragmatischen Einfluss ist fürs Erbrecht. Und da habe ich einen Gegenaufsatz

$18 \mathrm{zu}$ geschrieben. Einen langen Gegenaufsatz, um das in die richtige Richtung laufen zu lassen.

19 Das war auch mit Erfolg. Alle Stimmen, die ich bisher gehört haben, die haben sich meiner

20 Meinung angeschlossen.

The transition was for me ... My son is a psychiatrist, and he said to me that I had managed it astonishingly well. I managed it astonishingly well. I of course still had ... First of all, I have many hobbies, let's say. Running. Then philosophy. I do quite a lot of philosophy...

$21 \quad[\ldots]$

22 LB: Und was ist das denn mit Sport? Also was ist ... Was reizt Sie?

And what ist hat with sports?... What attracts you?

23 Prof. Dr Günther Schotten: Das ist dasselbe wie.- Sage ich jetzt mal, ich wiederhole jetzt 24 nur was meine Frau sagt, dass ist dasselbe wie mit dem Männlichkeitsgen. Leistungsbewusst,

25 Yanggüberschuss, sich beweisen, dass man das noch kann und so weiter. Das würde ich also 26 da unterordnen. 
That is the same as.- Let's say, I am just repeating what my wife says with the masculinity gene. Performance-oriented, Yang-excess, to prove oneself, that you can still do it and so on...

$1 \quad[\ldots]$

2 LB: Und sehr unterschiedlich. Sehr unterschiedliche Bereiche. ...And very different areas.

3 Prof. Dr Günther Schotten: Die Kammer ist Politik. Das habe ich dann wohl, nachdem ich

4 das acht Jahre intensiv gemacht habe, habe ich das dann aufgegeben, weil mir das zu viel

5 Politik war. Wissen Sie. Da wurde heute das gesagt, morgen das gesagt und übermorgen das

6 und das war alles nicht konsistent und... Da beschließt man was und dann weiß das am

7 nächsten Tag keiner mehr, was man beschlossen hat vorige Woche. Also...

The chamber is politics. I gave it up after I had done it intensively for eight years since there was too much politics. You know, they said this today, tomorrow something else and the day after that something else, that all was not consistent and...You decide something and then a day after nobody knows what had been decided the previous week.

$8 \quad[\ldots]$

9 LB: Großartig. Eine etwas verlorengegangene Tradition. Sowas gab es ja früher häufiger 10 durchaus auch an Unis. Aber das ist ja leider... Das war meine Vorstellung von 11 Philosophiestudium, aber das war schon zu meiner Zeit völlig... völlig illusorisch. Hat sich 12 denn... Wir haben ja eben darüber gesprochen, dass sich Ihre Identität durchaus auch an 13 Beruf orientiert hat und dadurch geprägt ist. Hat sich dann jetzt Ihre Identität verändert in 14 der Rente? Also dadurch, dass Sie Rentner sind und im Ruhestand?

Has your identity changed in retirement? Well, through being a pensioner and being retired?

15 Prof. Dr Günther Schotten: Nein, würde ich nicht sagen. Ich bin ruhiger geworden, weil 16 man auch zu wesentlicheren Fragen kommen kann. Mir sind auch viele Dinge gleichgültiger.

17 Auch in der Juristerei, in der Politik. Selbst in der Politik sind mir viele Dinge gleichgültiger

18 geworden. Also insofern würde ich das nicht sagen, dass sich etwas geändert hat.

No, I don't think so. I am calmer because you can concentrate on essential questions. I am more unconcerned about many things. In law, in politics. Even in politics, many things leave me more unconcerned. As such, I wouldn’t say that something has changed. 
1 LB: Also es hat Sie auch nicht... Also Sie fühlen sich jetzt nicht.. Wie soll ich das jetzt

2 ausdrücken? Sie haben nicht das Gefühl, dass Leute Sie anders wahrnehmen, weil Sie jetzt 3 im Ruhestand sind?

...You don't have the feeling that people perceive you differently because you are retired?

4 Prof. Dr Günther Schotten: Nein. Man ist natürlich im Beruf gefordert. Man tut ja etwas

5 für jemand anderes. Und man wird dann auch honoriert, aber das fällt natürlich weg. Das ist

6 also,.. Diese Beziehungen sind natürlich auch schon wesentlich für mich gewesen.

No. Of course, you are challenged in your job. You do something for somebody else. And then you are also rewarded, but that of courses ceases to exist. That is, well, ...These relationships were, of course, essential to me.

7 LB: Und fehlen Ihnen? Oder womit ersetzen Sie es?

And do you miss it? How do you substitute it?

8 Prof. Dr Günther Schotten: Gut, ich habe zwei Kolleginnen. Eine aus Köln, eine aus

9 Leverkusen. Die rufen mich regelmäßig an und suchen ihre Probleme zu lösen. Eine

10 Kollegin aus Leverkusen deren Mann ist auch Notar, die leben in einer Sozietät. Die kann

11 aber mit ihrem Mann nicht diskutieren. Die sind auf einer verschiedenen Wellenlänge. Die

12 ruft mich immer an. Wir sind auf einer absolut identischen Wellenlänge. Wenn die also

13 Fragen hat, sind die immer begründet. Ich finde auch meistens auch Lösungen. Insofern

14 heute Morgen hat auch eine Kollegin aus Köln angerufen. Insofern bin ich also noch dabei.

15 Aber das fehlt mir nicht mehr, nein. Das fehlt mir nicht mehr.

We are on an absolutely identical wavelength. When she has questions they are always absolutely justified...As such I am still in the game. But I don't miss it. I don't miss that anymore.

$16[\ldots]$

17 LB: Haben sich denn Ihre Prioritäten verändert? Also Sie haben es eben schon so angedeutet. Have your priorities changed?...

18 Prof. Dr Günther Schotten: Ja, meine Prioritäten haben sich natürlich schon verändert.

19 Weil ich natürlich jetzt, a) mehr Zeit für andere Dinge habe und diese natürlich auch sehr

20 viel Zeit beanspruchen, die ganzen Sachen. Deswegen hat man erst Recht nie Zeit ( lacht).

21 Das ist ein Problem. Man hat immer irgendetwas. 
Yes, my priorities have naturally changed somewhat. Because, of course, now I a)have more time for other things and these, of course, also take up a lot of time, these things. That's why you have no time again (laughs). That is the problem. There is always something.

$1 \quad$ LB: Also für Sie.- Also wenn man es zusammenfasst für Sie ist dieser Ruhestand von der

2 Qualität her durchaus ein Fortschritt?

3 So if you summarised retirement, is it with reference to quality an advancement?

4 Prof. Dr Günther Schotten: Ja, ja kann man wohl sagen.

Yes, you can absolutely say so.

$5 \quad[\ldots]$

6 LB: Ja, weil eine Qualität, ich glaube das ist immer das Entscheidende, wenn man neue

7 Lebensphasen, dass man die Qualität zumindest beibehält, die man vorher hatte.

Yes, because the quality is I believe the crucial thing when you begin a new life stage, that you at least keep the level of quality you had before.

8 Prof. Dr Günther Schotten: Ja und es hängt natürlich auch davon ab, wenn man jetzt sehr 9 viel liest und sehr viel diskutiert zu Hause. Und ja meine Frau und ich, wir haben also eine...

10 Wir verstehen uns auch intellektuell zu $100 \%$. Also sind fast immer ähnlicher Meinung. Also

11 auch kritisch, sehr kritisch. Wir sind also richtige Individualisten eigentlich. Aber sehr

12 kritisch auch gegenüber allem und nichts und insofern ist das schon ganz gut gewesen.

And yes, my wife and me, we have well, ...We also get on intellectually $100 \%$. We are nearly always of the same opinion. Also critical, very critical. We really are individualist somehow. But also very critical about all and nothing and as such it $^{27}$ has been quite good.

$13[\ldots]$

14 LB: Gibt es denn- Wir haben relativ viel jetzt geredet über positive Aspekte des Alters. Wie 15 sieht es denn aus mit negativen Aspekten beziehungsweise Einschränkungen durch Alter?

16 ... How about the negative aspects of older age respectively limitations by older age?

17 Prof. Dr Günther Schotten: Bisher habe ich die noch nicht. Meine Frau ist gesund. Ich bin 18 gesund. Wir reisen viel. Können auch noch... Wir werden auch in der Regel erheblich jünger 19 geschätzt als wir sind. Also insofern habe ich da noch keine Einschränkungen. Wir merken 20 zwar auch jetzt beim Wandern... Ich bin mit meinem Sohn acht Tage in Südtirol jetzt

${ }^{27} \mathrm{It}=$ retirement respectively the quality of life during retirement 
wandern gewesen zusammen. Das machen wir seit sieben Jahren. Ganz schön. Eine ganze

2 Woche immer. Ich merke beim Aufstieg bin ich noch richtig gut fit und beim Abstieg bin ich etwas unsicherer. Das ist also die erste Einschränkung, die ich so... und auch Gedächtnis lässt nach. Das ist ohne Zweifel. Also Gedächtnis lässt nach.

My wife is healthy. I am healthy. We travel a lot. We also have the ability to...People usually think that we both are considerably younger than we are...I have been noticing that uphill I am still really good, but downhill I am feeling a little bit insecure. That is, therefore, the first limitation, that I ... and also the memory declines. That is without a doubt, so the memory is declining... but very moderately and peu-à-peu only. Well, I would say so far I don't feel any limitations due to older age.

5 LB: Aber sehr...

But very...

6 Prof. Dr Günther Schotten: Aber sehr bescheiden und peu à peu nur. Also ich würde sagen

7 bisher spüre ich noch keine Einschränkungen des Alterns.

But very moderately and only peu à peu. Well, I would say, so far I don't experience any limitations due to older age.

8 LB: Und denken Sie denn, dass es etwas damit zu tun hat, wie Sie leben?

And do you think this has something to do with your way of life?

9 Prof. Dr Günther Schotten: Ja. Ja. Man ist natürlich... Man weiß natürlich nicht, vielleicht 10 ist man auch ungerecht, ja, das andere Leute... wir leben seit vielen Jahren konsequent 11 gesund. Wir gehen eigentlich so gut wie nie zum Arzt und haben eine Krankenkasse, wo wir 12 seit 22 Jahren keine Arztrechnung eingereicht haben.

Yes, yes... we have been living consequently healthy for many years. We hardly ever go to see the doctor and are members of a health insurance in which we haven't given in a doctor's invoice for 22 years. ...

13 LB: Das ist ja unglaublich.

That is unbelievable.

14 Prof. Dr Günther Schotten: Wir haben eine hohe Eigenbeteiligung. Deswegen lohnt es 15 sich auch nicht. Aber und wir leben eigentlich vegetarisch. Und essen sehr, sehr einfach.

16 Und konsequent und versuchen sehr gesund zu leben. Meine Frau kauft im Bioladen ein, 
1 ganz konsequent. Also insofern glaube ich schon, dass es auch was mit der Lebensweise zu

2 tun hat.

We really live vegetarian. And we eat very, very plainly and simply. And consequently and try to live healthily. My wife buys in the health-food shop, very consistently. As such I believe that it also has something to do with the life-style... Occasionally I like drinking a glass of wine. As such, I also treat myself, but it has to be an exception. When you do it regularly, then you don't treat yourself anymore. Then it is routine.

$3 \quad[\ldots]$

4 LB: Wenn Sie so in die Zukunft blicken, gibt es Dinge, die Sie ängstigen?

If you look into the future, are there things which worry you?

5 Prof. Dr Günther Schotten: Mich ängstigt eigentlich die Entwicklung der Welt. Der 6 ganzen Welt. Die Welt brennt ja an allen Ecken und Enden. Und wenn man denkt...wir 7 haben zwei Enkelkinder, wenn man denkt, die sind jetzt acht und sechs ein halb, wenn man

8 denkt was auf die zukommt. Da ängstigt uns schon, muss ich ehrlich sagen. Denn ich bin da 9 sehr, sehr skeptisch..

I am worried about the development of the world. The whole world. The world is going up in flames everywhere. And when you think ... we have to grandchildren and when you think, they are now eight and six and a half, when you think of what is coming their way. That worries us, honestly. Since I am very, very sceptical ...

$10 \quad[\ldots]$

11 LB: Heißt.- Was denken Sie denn, was das generelle Problem ist? Also... ...What do you think the general problem is? Well...

12 Prof. Dr Günther Schotten: Ja, das generelle Problem ist glaube ich schon, dass eine 13 westliche Welt über Jahrhunderte lang im Endeffekt die ganze andere Welt radikal 14 ausgebeutet hat. Radikal ausgebeutet hat und das nicht wieder einholen kann. Also es ist 15 nicht damit getan, jetzt zu sagen, wir müssen was für Afrika tun, wenn über Jahrhunderte so 16 ein Land ausgebeutet hat in einer Form, wobei Deutschland ja da noch relativ wenig mit zu 17 tun hatte, weil wir aus den Kolonialmächten früh verdrängt worden sind, aber sonst wären 18 wir auch darin Meister geworden. Also insofern bin ich sehr, sehr skeptisch.

Yes, the general problem is, I believe, that the western world has radically exploited the entire rest of the world for centuries. It has exploited the world radically, and it can't redeem 
that. It is not enough to say now we need to do something for Africa when this country has been exploited for centuries...

$1 \quad[\ldots]$

2 LB: Der ist natürlich auch ein großer Ehrgeizling und natürlich ist er da... Da kann man sich

3 schon vorstellen, wie der so im Hintergrund: „Wie komm ich wieder ran?“ Ja, ich könnte

4 noch stundenlang.. Es ist wirklich sehr interessant mit Ihnen, aber ich muss wieder zurück

5 auf dieses Altersthema. Sind Sie gläubig?

... Do you believe in God?

6 Prof. Dr Günther Schotten: (Zögert) Nein. Nein. Nein. Ganz ausschließen will ich das 7 nicht. Ich bin also im philosophischen Sinne gläubig, dass ich glaube. Das es Glaube, dass 8 es mehr gibt als nur Mechanismus der Natur, sage ich jetzt mal. Es ist auch ganz interessant 9 welchen Ansatz man hat. Da sind wir auch wieder bei Kant. Kant hat das sehr schön 10 herausgearbeitet, dass es einmal die Naturgesetzte gibt und wenn man die Naturgesetze 11 immer nur streng anwendet und nur die Naturgesetzte sieht man eigentlich den wesentlichen 12 Aspekt der Natur, nämlich die Schönheit der Natur, die schöne einer Blume, die Schönheit 13 einer Wiese, die hat man völlig aus dem Spiel. Die hat man völlig aus dem Spiel. Und dann 14 kommt etwas, was mehr ist als nur Naturmechanismus und insofern, ich kann mir also nicht 15 vorstellen, dass es so ein Jenseits gibt wie es die Kirchen vertreten. Das kann ich mir nicht 16 vorstellen.

I don't want to rule that out totally. I am therefore in the philosophical sense a believer since I believe. That is a belief that there is more than natural mechanisms, let's say...

17 LB: Ja das ist halt auch ein bisschen...Auch irgendwie etwas... Ich finde auch die Kirchen...

18 Das ist das Problem, was ich immer mit Kirchen habe, dass die so viel mit Angst arbeiten.

19 Wobei Himmel okay, wobei könnte ich mir auch schwer vorstellen, aber Hölle.

...That is the problem I have with the church that they work with creating fear so much...

20 Prof. Dr Günther Schotten: Zu Himmel braucht man ja ein Gegenspiel. Also wenn jeder 21 in den Himmel kommt, habe ich keine Angst mehr. Aber da kann ich nur mit Kant darauf antworten, wenn ich nur ein Strafsystem habe und das ethisch begründen will, das ist das Prinzip der Unsittlichkeit schlechthin. Hat Kant gesagt. Das ist genau richtig. Das ist das Prinzip der Unsittlichkeit schlechthin. Man darf überhaupt damit nicht arbeiten. Die Ethik muss genau an die Vernunft appellieren. An die Autonomie, dass der Mensch fähig ist auch richtig zu handeln, wenn er es auch selten tut. 
...Ethics has to appeal to rationality. To the autonomy, that the human is able to act correctly, even though he seldomly does so.

$1 \quad[\ldots]$

2 LB: Dann würde das ganz anders aussehen, aber wenn man eben ununterbrochen sich nur

3 in Geboten bewegt und Verboten.

Then it would be totally different but if you only move within commandments and restrictions.

4 Prof. Dr Günther Schotten: Und auch im Kleinmachen. Das ich nicht würdig bin, dass du

5 eingehst unter meinem Dach ist... Sie sind oder waren evangelisch? Ich bin katholisch

6 gewesen. Bin aber aus der Kirche ausgetreten. Bin aber wieder eingetreten auf Wunsch

7 meiner Frau, obwohl meine Frau evangelisch ist. Aber im katholischen gibt es zum Beispiel

8 "Du bist nicht würdig, dass du eingehst unter meinem Dach". Das ist vor der Kommunion.

9 Da wird der Mensch so klein gemacht. Da wird der Mensch so klein gemacht. Das ist ein

10 völlig verkehrter Ansatz. Und der führt eben auch dazu, dass die Menschen unmündig

11 werden und...

...The human being is made to look so small. That is a totally wrong approach. And that leads to humans being reduced to someone who is under age and...

$12[\ldots]$

13 LB: Das muss man leider so sagen. Das führt nicht unbedingt dazu, dass es populärer wird.

14 Also das ist einfach so. Und ich meine, natürlich sind die auch sehr, einfach sehr rückständig.

15 Setzen Sie...Ich meine das ist ja einer der Dinge, die je älter man wird doch auch immer

16 näher rückt... setzen Sie sich mit dem Tod auseinander?

...do you think about death?

17 Prof. Dr Günther Schotten: Ja. Ja.

Yes, yes.

18 LB: Ja?

Yes?

19 Prof. Dr Günther Schotten: Ja. Ich habe zurzeit überhaupt keine Angst vor dem Tod. Ich

20 habe nur Angst vor dem Sterben. Ich hoffe, dass... Ich bin kein Freund der aktiven

21 Sterbehilfe, muss ich ganz ehrlich sagen. Obwohl die Abgrenzung schwierig ist. Aber passive

22 Sterbehilfe halte ich für absolut notwendig. Das heißt, wenn einer Schmerzen hat, dass man 
1 Schmerzmittel gibt, selbst auf die Gefahr hin, dass es das Leben kostet. Ja, weil es ja dann

2 auch kein sinnvolles Leben mehr ist. Aber ein aktives Einschreiten, das würde ich für ganz

3 gefährlich halten. Das gibt einen Dammbruch. Dann muss man nachher mit 75 oder 80 zum

4 TÜV. (beide lachen) Und dann heißt es, Sie dürfen oder Sie dürfen nicht mehr.

At the moment I'm not afraid of death at all. I am only afraid of the process of dying. I hope that...I am not in favour of actively assisted suicide; I have to say honestly. Although the differentiation is difficult. But passively assisted suicide I believe to be necessary. That means when somebody is in pain, that one provides pain killers even if that could cost that person's life. Yes, because that is then no meaningful life anymore...

$5 \quad[\ldots]$

6 LB: Also das habe ich meinen beiden Eltern gewünscht und das wünsche ich auch mir, dass

7 man einfach in einer gewissen Würde stirbt und ich meine, wenn sich das länger hinzieht

8 dann ist es nicht mehr so weit her mit der Würde und das ist einfach so. Haben Sie.- Nur

9 nochmal einmal zurück, weil Sie gesagt haben, Sie haben Angst vor dem Sterben aber nicht

10 vor dem Tod-

... you are afraid of dying but not of death-

11 Prof. Dr Günther Schotten: Vor dem Sterbeprozess. Das ich leiden muss.

Of the process of dying, that I have to suffer.

12 LB: Und das bezieht sich auf körperliche Schmerzen oder eher den Verlust von Autonomie? And that refers to physical pain or more the loss of autonomy?

Prof. Dr Günther Schotten: Ach, den Verlust von Autonomie, den bekommen Sie dann ja

14 nicht mehr mit. Wenn der Verlust da ist. Das ist ja glaube ich nicht mehr das Problem, weil

15 wenn er wirklich voll da ist. Aber der Verlust von Autonomie ist natürlich schon schwierig.

16 Ich habe diesen Freund, von dem ich eben erzählt habe, der diese Kant-Zitate bei Helmut

17 Schmidt immer hineingebracht hat. Der hat möglicherweise durch einen ärztlichen

18 Kunstfehler, 81, 82, seitdem ist der querschnittsgelähmt. Und der hat sich seit 20 Jahren zum

19 ersten Mal jetzt wieder bei mir gemeldet. Ich habe ihn zweimal besucht und heute Morgen

20 auch noch mit ihm gesprochen und werde ihn in den nächsten Tagen wieder besuchen. Da

21 würde ich natürlich auch Angst vor körperlicher Autonomie haben. Obwohl das ist mir

22 bisher nicht so bewusst, dass das akut werden könnte. Ich habe eigentlich nur Angst vor

23 einem zu langen Sterbeprozess mit vielen Schmerzen und sonst irgendwas, obwohl das ja

24 angeblich relativ stark eingedämmt werden kann, habe ich gehört. Ich weiß es nicht. 
Well, you won't then notice the loss of autonomy. When the loss is there... But the loss of autonomy is, of course, problematic.

1 LB: Ja, dafür ist es natürlich auch wichtig, dass man die richtigen Ärzte hat. Und ein gutes

2 Verhältnis hat und all solche Dinge.

Yes, for this it is, of course, important that you know the right doctors. And that you have a good relationship with them and such things.

3 Prof. Dr Günther Schotten: Genau.

Exactly.

$4 \quad$ LB: Haben Sie denn noch Ziele, Wünsche für die Zukunft?

Do you have aims, wishes for the future?

5 Prof. Dr Günther Schotten: Ja, ich habe noch den Wunsch Marathon unter vier Stunden 6 zu laufen.

Yes, I have the wish to run a marathon under four hours.

7 LB: Das habe ich doch schon... Das habe ich doch schon gehört. ... I heard about this already.

8 Prof. Dr Günther Schotten: Heute morgen bin ich 15 Kilometer in dem Tempo gelaufen,

9 aber ich bin in Paris am 9. April wollte ich bis Kilometer 25, war ich auch in der Zeit, aber

10 dann bin ich total eingebrochen. Dann habe ich ganz lange gebraucht. Da habe ich eine halbe

11 Stunde länger gebraucht. Weil ich nachher gar nicht mehr konnte. War auch zu heiß. War

12 fast so heiß wie heute. Das ist schlecht für Marathon. Also insofern habe ich noch... Das ist

13 ein schizophrener Wunsch, das weiß ich, aber es ist einer.

That is a schizophrenic wish; I know that, but it is one.

$14 \quad[\ldots]$

15 LB: In dieser, ja, etwas schwierigen Welt im Augenblick. Denken Sie, hat sich in den letzten,

16 ja es ist bei Ihnen ja wirklich noch nicht so lange, aber hat sich seit dem Ruhestand denn Ihre

17 Weltsicht in irgendeiner Form verändert?

...Has your view of the world changed since your retirement?

18 Prof. Dr Günther Schotten: Nein, würde ich sagen nicht.

No, I wouldn't think so. 
1 LB: Kontinuum. Also hat sich weiter fortgesetzt?

Continuum. So it continued?

2 Prof. Dr Günther Schotten: Hat sich weiter fortgesetzt. Führt...Hat sicherlich auch zu

3 wesentlicheren Einsichten geführt. Also das man auch Wesentlicheres für wesentlich hält.

4 Und dass man sich auch fragt, wieso man sich früher über manche Dinge geärgert hat. Aber

5 das ist allgemeines Leben.

... Has certainly also led to essential insights. Well, that to perceive what is essential as essential. And that you also ask yourself why you got angry about some things in previous times...

6 LB: Was ist denn... Also Sie haben es vorhin schonmal erwähnt, was ist denn wesentlich? ...what is essential?

7 Prof. Dr Günther Schotten: Wesentlich ist für mich eine gewisse innere Ruhe mit einem

8 philosophischen Ansatz. Und das man also zu den Dingen kommt... Das man ernsthaft

9 diskutieren kann über Grundfragen des Lebens und dazu zählt auch als Frage zumindest,

10 Glaube. Gar kein Zweifel. Ohne Antwort. Und so viele Antworten gibt es ja überhaupt nicht.

11 Es gibt viel Fragen und wenig Antworten. Aber das man da intensiv und gut... Ich meine,

12 meine Frau und ich verstehen uns ausgesprochen gut. Haben auch einen guten Austausch.

13 Wir reisen auch noch relativ viel. Also insofern... aber so ganz konkret sind außer diesem

14 etwas schizophrenen Ziel, Marathon unter vier Stunden-, ...

Essential for me is a certain inner tranquillity with a philosophical basis...

15 LB: Aber warum denn schizophren?

But why, schizophrenic?

16 Prof. Dr Günther Schotten: Gut, ich meine da fragt kann man sich natürlich, ob das sein 17 muss.

Well, one could, of course, ask, if this must be...

18 LB: Ja, aber das ist schon auch.- Das sind jetzt auch die letzten Fragen von mir, aber das ist

19 ja auch irgendwo sowas gesellschaftliches, dass man irgendwie sagt ja, also wenn das ein

20 40-jähriger sagen würde, würde man sagen, völlig normal. Aber dann in Ihrem Alter sagt 21 man...

...Well, if a 40-year old said that, one would say, totally normal. But in your age one says... 
1 Prof. Dr Günther Schotten: Es ist natürlich so, ich habe immer geträumt früher mal unter

2 drei Stunden zu laufen. Habe ich nicht geschafft. Ich bin auf drei Stunden und sechs Minuten

3 gekommen und dann ist es weniger geworden, weil ich nach Köln gegangen bin. Und dann

4 die Laufgruppe weg war. Und dann bin ich nicht mehr so viel gelaufen. Und man ist

5 natürlich, wenn man... man sagt zum Beispiel jedes Jahr wird man auf 10 Kilometer eine

6 Minute langsamer im Alter. Ab einem gewissen Alter und das ist natürlich schon was und

7 das stimmt jetzt irgendwo, wenn ich also denke, dass ich um die drei Stunden vor 30 Jahren

8 gelaufen bin und jetzt komme ich an die vier Stunden nicht ran. Das ist natürlich schon...

It's, of course, like this, I had previously always dreamt of running a marathon under three hours. I didn't manage it. I managed three hours and six minutes... And then I didn't run so much anymore. And of course, when you...it is said that with increasing age you become one minute slower per ten kilometres every year. At a certain age and that is, of course, something and that is somewhat true if I think that I ran about three hours thirty years ago and now I can hardly reach four hours. That really is ...

9 LB: Das ist dann ein relativ hochgestecktes Ziel.

It is then quite an ambitious aim.

10 Prof. Dr Günther Schotten: Ja, für mein Alter ist das ein relativ hochgestecktes Ziel.

Yes, an ambitious aim for my age.

$11[\ldots]$

12 LB: Das ist sehr gut, weil die nächste Frage bezieht sich nämlich tatsächlich auf jüngere

13 Menschen. Haben Sie den Eindruck, dass jüngere Menschen... Also jünger, Menschen, weiß

14 nicht 20-, 30-, 40-, 50-jährige sich Ihnen gegenüber anders verhalten als gleichaltrigen oder 15 jüngeren?

...Do you have the feeling that younger people...behave differently towards you than towards people their age or younger?

16 Prof. Dr Günther Schotten: Nein. Bisher nicht.

No. Not so far.

17 LB: Da ist nicht dieser Respekt?

There isn't this kind of respect?

18 Prof. Dr Günther Schotten: Nein, bisher nicht. 
No, not so far.

$1 \quad$ LB: Würden Sie sich das wünschen?

Would you wish for it?

2 Prof. Dr Günther Schotten: Auch nicht, nein, nein. Also ich habe eigentlich nicht das

3 Gefühl, dass ich irgendwo nicht respektvoll behandelt worden wäre. Also das... Ich erinnere

4 mich zwar einmal als ich in Bielefeld zur Uni gefahren bin in der U-Bahn und da gab es

5 keinen Platz, da ist eine Studentin für mich aufgestanden. Das habe ich aber abgelehnt. Das

6 hat mich etwas irritiert und dann habe ich nachher realisiert, dass das eine Studentin aus dem

7 eigenen Kurs war. Deswegen weiß ich ja nicht, ob sie es auch sonst bei einem älteren Mann

8 gemacht hätte. Aber... Gut manchmal denke ich natürlich schon, wenn man so 10- 12-

9 jährige in der Straßenbahn oder im Zug sich rumfläzen sieht und da denkt keiner für einen

10 älteren Menschen mal aufzustehen. Das irritiert mich schon. Das muss ich ganz ehrlich

11 sagen. Aber das bezieht sich dann nicht auf mich persönlich sondern ganz allgemein. Das

12 man sagt, das ist eigentlich schlecht.

Also, no, no, no. Well, I don't really have the feeling that I am not treated with respect somewhere. Well, this...I remember, however, that one time when I was on my way to the university in Bielefeld with the tram, and there was no vacant seat, then a female student got up for me. I rejected that, however. It irritated me slightly, but then I realised later that she was a student in one of my courses. That's why I don't know if she had done that also for another older man. But...well, sometimes I obviously think when you see 10-12-year-old teenagers on the tram or on the train, and you see how they sprawl and nobody thinks of getting up for an older person. That does annoy me. I honestly have to say. But that doesn't refer to me but in general. That you say, that really is bad.

13 LB: Und das Gegenteil? Ein hoher Respekt, der Ihnen entgegengebracht wird? Erleben Sie 14 das aufgrund Ihres Alters?

And the opposite? A high respect that is paid to you? Have you experienced that due to your older age?

15 Prof. Dr Günther Schotten: Auch eigentlich nicht.

Not really.

16 LB: Würden Sie es angemessen finden?

Would you find that appropriate? 
1 Prof. Dr Günther Schotten: Och, weiß ich nicht. Weiß ich nicht. Wenn ich das Gefühl

2 hätte wegen des Alters respektiert zu werden, das wäre mir zu wenig.

Not really, no. ... I don't know. I don't know. If I had the feeling that I was respected simply because of my age, that would be too little for me.

3 LB: Ja. Haben Sie denn... Denken Sie das Altern für Frauen schwieriger ist als für Männer? ...Do you think that ageing is more difficult for women or for men?

4 Prof. Dr Günther Schotten: Habe ich gehört, dass das für Frauen schwieriger sein soll. I heard that it is more difficult for women.

5 LB: Denken Sie, es ist wahr?

Do you think that is true?

6 Prof. Dr Günther Schotten: Ich weiß es nicht. Ich glaube es nicht. Bei meiner Frau ist das 7 nicht der Fall. Die hat genauso wenige Probleme mit dem Älterwerden wie ich. Man hört es 8 regelmäßig, aber in meinem Bekanntenkreis kann ich das eigentlich nicht verifizieren, dass 9 das so ist.

I don't know. I don't think so. It's not the case with my wife. She as little a problem with getting older as me. You hear that regularly but in my circle of friends, I cannot confirm that.

$10 \quad[\ldots]$

11 LB: Generell wie würden Sie unsere Einstellung in der Gesellschaft zum Alter beschreiben? In general, how would you describe the attitude of our society to older age?

12 Prof. Dr Günther Schotten: Die ist nicht allzu positiv. Ja, die ist nicht allzu positiv. Das 13 können Sie auch daran erkennen, dass zum Beispiel 50- 60-jährige Schwierigkeiten haben 14 einen Arbeitsplatz zu finden. Ja, ich habe das nie nachvollziehen können. Ich habe eigentlich 15 immer relativ alte Mitarbeiter gehabt und war immer bestens zufrieden. Also dieser 16 Jugendwahn, der da in vielen Fällen gemacht wird, in vielen Firmen auch, den finde ich also 17 für jetzt nicht für die 60, 70, 80-jährigen, aber für die 50-, 60-jährigen finde ich die schon 18 problematisch. Da wird also... Da zählt man zu schnell zum alten Eisen. Das finde ich eine 19 ganz schlechte Entwicklung. Ist aber auch eine typisch egoistische Entwicklung.

It is not particularly positive. Yes, it is not very positive. You can see that, for example, in the difficulty, 50-60-year-old people have to find employment. Yes, I have never understood that. I always had quite old employees, and I was always very happy with them. Well, this 
obsession with youth, that is applied in many cases, also in many companies, I find, well not for the 60,70 or 80 years old people but for the 50, 60 years old people, I find that problematic. There it becomes, well, ... you are viewed as belonging to the scrap heap quickly. I find that a very bad development. But it's a typically selfish development.

1 LB: Ah. Das ist meine nächste Frage. Wo kommt das her? Worauf basiert diese negative

2 Konnotation in unserer Gesellschaft, die man mit dem Alter verbindet?

What are these negative connotations linked to aging based on?

Prof. Dr Günther Schotten: Ich glaube das liegt an einem immer stärker werdenden Egoismus der Gesellschaft. Das merken Sie an allem ... Rücksichtslos in einer Form, die man auch nur bewundern kann manchmal.. wie rücksichtslos Leute sind. Ich habe jetzt noch gerade.- Wir sind ICE gefahren zu einem Heilpraktiker, der früher in Monschau war und der

7 ist nach Österreich gezogen. Da sind wir letzte Woche in Österreich gewesen und da ist in 8 Mainz jemand im Zug eingestiegen. Der fuhr bis Nürnberg. Also der hat da einen reservierten Platz neben dem Sitz meiner Frau. Der ist, ich schätze 40. Der hat seinen Laptop aufgenommen, nur telefoniert in einer aggressivsten Form. Hat nicht "Guten Tag" gesagt. Nichts. Sich breitgemacht. Und nach Nürnberg waren wir dann richtig froh. Ich bin zum Teil weggegangen, weil ich das nicht mehr ertragen konnte. Hat auch in Englisch gesprochen und auch sehr viel Oberflächliches. Und gestört, gestört und dann steht er in Nürnberg auf.

14 Meinen Sie der würde „Auf Wiedersehen“ sagen? Einfach so. Das ist so ein Ausdruck unserer Zeit. Wollte ich mal sagen. Das ist also etwas was ganz stark ist oder auch zum Beispiel, dass die Leute sich immer beschweren. Ich bin gestern bei meiner Tochter gewesen, da haben sich die Lehrer beschwert, dass die Eltern immer gleich bei der kleinsten Kleinigkeit mit einem Anwalt drohen. Wenn das Zeugnis nicht so ist, wie sich die Eltern das vorstellen, dann drohen die gleich mit dem Anwalt. Oder auch im Notariat zum Beispiel, da gab es in den ersten zehn, elf, zwölf Jahren, da habe ich kein einziges Mal erlebt, dass sich jemand über eine Kostenrechnung beschwert hat. Die wurde akzeptiert. Vielleicht zu autoritätsgläubig. Heute ist das Gang und Gebe. Fast bei jeder Kostenrechnung fragen die Leute nach, wieso ist das denn so?, und warum das denn?, und so weiter. Das sind so ein Ausdruck der... Oder auch wenn immer alles um jeden Preis billig werden muss. Das ist auch ein Ausdruck von Egoismus, der sich ganz stark breit macht, glaube ich.

I believe it is the result of ever-increasing selfishness in society. You can notice this in everything...Inconsiderateness in a way that you can somehow only admire sometimes...how inconsiderate people are... That is also an expression of selfishness that is expanding quickly ... 
1 LB: Und Egoismus auch verknüpft mit Produktivität?

And selfishness is linked to productivity?

2 Prof. Dr Günther Schotten: Ja, die Produktivität muss ja laufen, damit man eben auch alle

3 egoistischen Wünsche erfüllen kann. Also insofern bemüht man sich zum Teil auch

4 produktiv $\mathrm{zu}$ sein. Ich glaube schon, dass eine Gesellschaft sehr, sehr stark

5 ellenbogenförmig sich gebildet hat.

Yes, productivity has to run so that one can fulfil all of one's selfish wishes. Thus, one also partly tries to be productive. I do believe that an increasing dog-eat-dog society has developed.

6 LB: Und das heißt dann also.- Nur um das nochmal abzuklären mit dem Egoismus.-

7 Egoismus heißt auch gleichzeitig Konsum? Also seine eigenen Bedürfnisse befriedigen in 8 einem hohen Ausmaß?

...Selfishness simultaneously means consumption? So to satisfy one's own desires to a high degree?

9 Prof. Dr Günther Schotten: Also der Konsum ist ein übertriebener Konsum ist Ausdruck 10 eines Egoismus. Ein Zeichen dafür. Ein Symptom.

Well, consumption, excessive consumption is an expression of selfishness. A sign of that. A symptom.

11 LB: Sie haben ja durchaus auch ein bisschen Erfahrung mit anderen Ländern, haben Sie den

12 Eindruck, dass die Einstellung zum Altern in anderen Ländern anders ist?

...Do you have the impression that the attitude towards ageing is different in other countries?

13 Prof. Dr Günther Schotten: Habe ich eigentlich bisher nicht bemerkt. Ich habe auch ehrlich 14 gesagt mir noch nie auf so Reisen Gedanken gemacht, ob, wie so die Einstellung zum Alter

15 ist, weil ich mich selbst gar nicht so alt fühle. Ich fühle mich gar nicht so alt. Wenn ich das 16 selbst so realisiere, wie alt ich bin. Dann weiß ich wie alt ich bin aber ich fühle mich in dem

17 Sinne gar nicht so alt und deswegen die Einstellung da anders ist, weiß ich nicht. Ist mir 18 eigentlich nicht bewusst geworden.

So far, I haven't noticed any differences. I have honestly never thought about whether, how the attitude towards ageing is since I don't feel that old. I don't feel that old. When I realise how old I am, then I know how old I am, but in this way, I don't feel that old, and that's why I don't know if the attitude there is different. I have never really noticed. 
2 LB: Ganz genau. Das ist ja so ein bisschen das Problem.

Exactly. That is somewhat the problem.

3 Prof. Dr Günther Schotten: Wenn man nicht gesund ist, wenn man gehbehindert ist oder

4 querschnittsgelähmt, dann sieht die Situation ganz, ganz anders aus.

If you are not healthy, when you have a walking disability, or you are quadriplegic, then the situation looks totally, totally different...

5 LB: Natürlich hat das Ganze auch ein bisschen was damit zu tun wie man finanziell 6 aufgestellt ist.

The whole thing is also linked to what your financial situation is.

7 Prof. Dr Günther Schotten: Klar, eine gewisse finanzielle Absicherung ist ganz wichtig. ... a certain financial security is very important

$8 \quad[\ldots]$

9 LB: Jaja, das ist so nicht.- Also ich meine grundsätzlich.- Ich habe mich jetzt auch relativ

10 viel mich damit beschäftigt, wie das in den Medien dargestellt ist und so. In letzter Zeit gibt 11 es sehr, sehr viel Werbung mit diesen sehr aktiven älteren Menschen, was ja grundsätzlich 12 eigentlich ganz schön ist, aber ich eben denke, das schafft wieder so eine Erwartungshaltung.

13 Wo man dann denkt, aber nicht jeder ist ja gleich und nicht möchte irgendwie-

...there is a lot of advertising with very active older people lately, which is generally great but I think it also creates such expectations. Where you think, not everybody is the same and wants...

14 Prof. Dr Günther Schotten: Und jeder kann auch nicht so aktiv sein.

And not everybody can be so active.

$15 \quad[\ldots]$

16 LB: Letzte... Letzter Themenbereich, weil wir ja eben schon drüber gesprochen haben.

17 Mucki-Bude. Wie ist das denn? Gehören Muskeln für Sie zur Männlichkeit? ...Do muscles form part of your conception of masculinity?

18 Prof. Dr Günther Schotten: Allein schon deswegen nicht, weil ich keine habe.

No, simply because I don't have any. 
1 LB: Das heißt, gibt es physische Attribute oder gibt es etwas, wo sie sagen würden, das

2 gehört irgendwie zur Männlichkeit?

That means, are there any physical attributes or something like that that you would say that is part of masculinity?

3 Prof. Dr Günther Schotten: Ja gut, das kriegt ja man vorgelebt oder vorgezeigt.

Yes, well, that is exemplified and shown.

$4 \quad[\ldots]$

5 Prof. Dr Günther Schotten: Für mich abstoßend. Also kein Neidfaktor.

For me, this is repellent. So no factor for envy.

$6 \quad[\ldots]$ 


\section{Interview \& Translated Extracts GB}

2 LB: So ist das. Ja. Und das ist vielleicht der Horror, weil ich tippe mit zwei Fingern (lacht).

3 Okay, also ich habe dir ja diesen ganzen Kram vorher schon erzählt. Das heißt, du weißt,

4 worum es geht. Warte. Dann fangen wir auch direkt mit dem Entscheidenden an, nämlich,

5 was bedeutet Männlichkeit für dich?

\section{...What does masculinity mean to you?}

6 GB: Da habe ich mir ehrlich gesagt noch keine Gedanken drüber gemacht. Also,

7 Männlichkeit ist bei mir eigentlich so ein bisschen mehr mit Fragezeichen als mit

8 Ausrufezeichen besetzt. Weil ich war-, muss man natürlich die individuelle Situation sehen,

9 also wir waren zu vier Jungen zuhause, ich war der jüngste. Und war eigentlich, sage ich

10 jetzt mal, nicht nur qua Alter, sondern auch qua Wesen der zarteste von vieren. Und da die

11 anderen größer, stärker und raubeiniger waren, war ich so ein bisschen zarter besaitet, was

12 dann eigentlich, ja eher so vom Umfeld und für mich selber erlebt, ich will nicht sagen eher

13 weiblich angehaucht war, aber es war nicht diese Art Männlichkeit, sage ich jetzt mal, die

14 von anderen vorgelebt wurde. Ich war auch eher der musisch Begabte, während mein

15 nächstälterer Bruder war die Sportskanone, der spielte Fußball. Und davor der ältere war so

16 der Handwerker und der älteste war halt eben der älteste. Und insofern war Männlichkeit für

17 mich eigentlich immer so vorgelebt sage ich mal Stärke, Kraft, Dominanz, Machertyp. Aber

18 von mir selber eher so mit einem kleinen Fragezeichen versehen. Weil ich das von meinem

19 Naturell her gar nicht so war. Also, ich hatte eine Vorstellung, was Männlichkeit ist. Aber

20 auch sagen wir mal, ein Stück gesunder Zweifel daran, wie nachhaltig das ist und wie

21 berechtigt es auch ist, sage ich jetzt mal, einfach aus der Diskrepanz zu meinem eigenen

22 Empfinden, wie ich mich sah.

Honestly, I have never really thought about this. Well, to me masculinity is somewhat a little more linked to a question mark than to an exclamation mark. Since I was -, you need to see the individual situation, so we were four boys at home, and I was the youngest, and I was, let's say, not only by age but also by nature the most sensitive of the four. And since the others were bigger, stronger and rougher, I was somewhat more sensitive, what then somehow was, yes, somehow perceived, by my social surroundings more than by me, I don't want to say, to have a more feminine touch but it was not the kind of masculinity, let's say, that was embodied by others. I was also more artistically talented whilst my next oldest 
brother was the sporting ace, he played football. The one before that one was somehow the craftsman and the oldest was, well, the oldest. As such masculinity was somehow embodied by, let's say, strength, powerfulness, dominance, man-of-action type, but I somehow viewed this perception with a question mark since by nature I was not like this at all. So I had a conception of what masculinity was but, let's say, also some healthy doubts about how sustainable and also justified this was, let's say, simply because of the discrepancy to my own feelings, to how I viewed myself.

$1 \quad[\ldots]$

2 LB: Gibt es ja nicht. Und, hattest du eine bessere Beziehung zu deiner Mutter oder zu deinem 3 Vater?

...Did you have a better relationship with your mother or to your father?

4 GB: Ganz klar zu meiner Mutter. Ich war auch so ein bisschen ihr Nesthäkchen. Das hing 5 aber damit auch zusammen, weil ich als kleines Kind, da habe ich keine Erinnerung mehr 6 dran, das waren immer so Erzählungen, mal schwer krank war. Ich bin 1946 geboren, also 7 kurz nach dem Krieg und da war ich mal sehr schwer krank und lange im Krankenhaus und 8 es war so ein bisschen auf der Kippe. Und da muss meine Mutter fürchterlich gelitten haben

9 und deshalb war ich, war ihr auch sehr nah, also später, selbst als Erwachsener.

Clearly to my Mom. I was somehow her Benjamin... that is why I was; I was very close to her, also later, even as an adult.

10 LB: Ja. Und deine Eltern waren aber ihr Leben lang zusammen?

Yes, and your parents stayed together all their lives?

11 GB: Ja.

Yes.

12 GB: Also meine Mutter war auch das Zentrum der Familie. Mein Vater hat gearbeitet, von 13 morgens bis abends. Also, wir hatten ein Baugeschäft und der war körperlich von morgens 14 bis abends sehr stark engagiert. Und hatte aber immer noch, wenn er abends dann auf das 15 Lager kam, noch die halbe Stunde mit seinen Söhnen Fußball zu spielen und-. Aber meine 16 Mutter war die Seele der Familie. Die hat alles gemanagt, alles gemacht. Die war für alles 17 und jeden da. Ein Skorpion, ja, mit einem Riesenherz, aber auch einem großen Stachel, also 18 eine sehr wehrhafte Frau. Aber ihr ging nichts über die Familie. Und, also, ich meine, was 
man später, als Erwachsener denn, wenn man sich zurückerinnert, dann oft ein bisschen tendenziell anders sieht, für sie kamen die Kinder an erster Stelle und dann kam eine Zeitlang nichts, dann kam ihr Mann und dann kam der Rest der Familie. Aber ihr ganzer Stolz war, wenn wir sonntags geschniegelt und gespornt dann wie die Orgelpfeifen zur Kirche marschierten, schön sonntäglich angezogen. Und dann guckte sie uns nach und dann konnte man ihr ansehen, wie stolz sie war, dass ihre vier Bengel schön angezogen zur Kirche gingen.

Well, my mother was the centre of the family. My father worked from morning till night... and he still had when he came home the half hour to play football with his sons. But my mom was the soul of the family. She managed everything, did everything. She was there for everything and everybody. ... a well-fortified woman. But nothing took priority over her family. And, well, I mean, what you might as an adult when you look back, view a little more differentiated, for her, the children came first and then there was nothing for a while, then came her husband and then came the rest of the family....

7 LB: Junge, junge, vier Jungs.

...four boys.

8 GB: Ja, vier Jungs. Das war für meine Eltern auch, da insbesondere für meine Mutter, war 9 das eine Mammutaufgabe. Und das hat sie auch nie abgelegt. Das Highlight war, als wir 10 dann auch nacheinander in feste Bindungen gingen, obwohl meine Mutter immer gedroht 11 hat, dass jeder, der irgendwann heiratet, raus muss. Am Ende hingen wir alle vier, mit 12 unseren Frauen und der ersten Garnitur Kinder im Elternhaus. Alles unter einem Dach. 13 Meine Mutter hat angebaut, wo anzubauen war, um jedem, sagen wir mal, sein eigenes Reich zu schaffen, was dann auch funktionierte. Und da dann ja die Schwiegertöchter berufstätig waren oder studierten, war meine Mutter dann automatisch auch die Nanny für die erste

16 Generation Babys. So, und als alle nach den anfänglichen Ehejahren so viel Geld dann 17 verdienten, dass sie sich wohnungsmäßig auf eigene Füße stellen konnten, dann ging das so 18 ein bisschen auseinander. Und ich habe meine Mutter eigentlich nur im Kittel gekannt. Und 19 die hatte auch noch den ganzen Geschäftskram, Bürokram für die Firma gemacht. Es war 20 keine Riesenfirma, es waren vier Arbeiter. Aber alles, Schreibkram, Buchhaltung hat sie 21 dann gemacht. Und, also, ich habe keine Frau kennengelernt, die so gearbeitet hat, wie meine Mutter.

Well, I have never met a woman who worked as much as my mother. 


\section{Of course...}

2 GB: ... das ist Zukunft. Weil viel später, als arrivierter Topmanager, war das für mich eines

3 der schönsten Erlebnisse, dass ich meine Erfahrungen an junge Menschen weitergeben

4 konnte. Weil ich als junger Mensch erlebt habe, was es bedeutet, wenn du so einen, fast 5 schon auf dem Podest Stehenden vor dir hast, der sich aber dir zuwendet und der dir hilft 6 wie ein Vater. Also, ich habe dann relativ deutlich Karriere gemacht, ich war 7 Zentraleinkäufer, anfangs für Babyartikeln. Da hatte der Vorstand damals zu mir gesagt, als 8 er mich zum Zentraleinkäufer für Babyartikel ernannte: „Herr B., Sie haben zwei Aufgaben.

9 Die erste ist aus dem Bereich 20 Millionen mehr Umsatz zu machen, zweitens, noch ein 10 zweites Kind zu kriegen, damit Sie üben können (lacht). Und da habe ich gesagt, okay, das 11 zweite ist etwas leichter als das erste, aber da fällt mir auch noch was ein. Und das habe ich 12 dann auch geschafft. Dann habe ich den Lederwaren-Einkauf übernommen und dann wurde 13 ich Direktor für den gesamten Bereich Textil und war der jüngste Direktor in der Geschichte 14 der Kaufhalle. War dann plötzlich Chef von zehn Zentraleinkäufern, die vorher alle Kollegen 15 von mir waren und habe mich dann eigentlich selber gewundert, wie schnell ich eine solche 16 Akzeptanz und auch Respekt nicht qua Order de Mufti und nicht qua Stern auf der Schulter 17 erlangt habe, sondern einfach weil ich mit den Leuten so gut umgegangen bin und also sagen 18 wir mal, auf eine so offene und faire und selbstverständliche Weise, dass die gerne mit mir 19 gearbeitet haben. So. Und ja, und dann-.

...Because much later, as a parvenu top manager, that was for me the most delightful experience that I could pass on my experience to younger people. Since I experienced when I was a young person, what it means when one, who nearly stands on a pedestal in front of you, turns towards you and helps you like a father...

$20 \quad[\ldots]$

$21 \quad$ LB: Klar, aber wie hast du das geschafft zeitlich?

Clearly but how have you managed all that time-wise?

22 GB: Das habe ich zeitlich geschafft, weil ich eigentlich, das ist aber jetzt im Nachhinein, sag 23 ich mal, ja, auch ein Grund natürlich für den relativ tiefen Fall, ist der falsche Ausdruck, ist 24 ja kein Fall, es ist nur ein Wachwerden in einem völlig anderen Umfeld. Das Ganze, wenn 25 du die Story so hörst, dann waren zwei Dinge eigentlich signifikant für die spätere Entwicklung. Ich war international unheimlich viel unterwegs, deshalb konnte ich eigentlich 
nie, wie man so schön sagt, soziale Wurzeln irgendwo aufbauen. Denn, fünf Jahre Hong

2 Kong, dann sieben Jahre Schweiz, dann neun Jahre Deutschland, dann wieder 8 Jahre

3 Schweiz, zwischendurch mal schnell für ein halbes Jahr China oder ein Vierteljahr Korea, ist viel Wechsel. Für mich gab es eigentlich nur Job und am Wochenende Familie. Ich hatte keine Hobbies, für Hobbies hatte ich keine Zeit. Weil ich ja in der Woche in meinem Job eigentlich open end war, habe ich also ganz bewusst immer das Wochenende mir freigehalten, um mit den Kindern was zu unternehmen. So. Das hieß aber in der Konsequenz, ich hatte kein großes Network außerhalb des Jobs und ich hatte auch nicht irgendwelche Mitgliedschaften in Vereinen oder Hobbies, wie Tennis oder Golf oder was auch immer. Wo du sagen kannst, dann hast du ein paar Leute, die sind auch da, wenn du mal viel Zeit hast, so wie jetzt. Und das war dann schon im Nachhinein sehr, sehr schwierig. Was für mich eigentlich das große Problem war in der Umstellung, wobei natürlich dann meine Krankengeschichte dann dazukommt, das ist noch eine eigene Dimension. Aber das Schwierigste war eigentlich von jetzt auf gleich festzustellen, dass du eigentlich in einem ganz anderen Leben bist. In einem ganz anderen Leben. Wenn du Anwalt bist oder Arzt bist, dann kannst du eigentlich so lange arbeiten, wie du kannst. Wenn du leitender Angestellter bist oder Manager und in einem Unternehmen, wo wirklich dann mit 65 der Hammer fällt, dann ist das völlig egal, wie gut du bist und wie viel deine Erfahrung über 40 Jahre Handel, national und international, wert ist. Das wird einfach nicht mehr gebraucht oder das ist nicht mehr gewollt. Ich habe damals gesagt, nicht aus eigenem Interesse, sondern weil ich zutiefst davon überzeugt war, das war noch während meiner aktiven Zeit, nicht als Pensionär, Leute, warum gründen wir nicht einen Verein oder eine Firma in der Schweiz, die sind ja immer noch mit der Holding in der Schweiz oder zumindest mit der Gesellschafterholding in der Schweiz, wo wir pensionierte Topmanager einbinden als Pool, wenn irgendwo plötzlich ein Loch entsteht oder irgendwo einer von draußen reinkommt als Quereinsteiger in eine Vorstandsposition, dass wir Coaches haben, die denen helfen können. Keiner dieser Coaches darf jemand sein, der mit dem Verständnis kommt, ich beweise denen, dass ich immer noch besser bin. Sondern, Coaches mit dem Verständnis, je schneller ich mich überflüssig mache, weil der drin ist im Job, je besser ist es für mich. So. Ich wäre so einer gewesen. Ich kenne andere, die das genauso gesehen hätten. Dass die Firma das nie gemacht hat, kann ich nicht verstehen. Ich sehe oder habe x Beispiele gesehen, wo die Firma dann viel Geld ausgegeben hat für externe Beratungsunternehmen, wo du sagen kannst, die ersten, sagen wir mal, zwei, dreihunderttausend Euro sind mal gezahlt worden bis die überhaupt verstanden haben, wie unser Geschäft funktioniert. Und das hätte man sich sparen können. Also ich habe Verständnis dafür, dass junge Leute, die in Management-Positionen kommen, einen Horror vor alten Seilschaften haben und vielleicht sagen, um Gottes Willen, wieder so ein alter 
1 Dinosaurier, der meint, das Geschäft so wie es vor 20 Jahren war, wäre auch heute noch, und

2 die keine Beratung wollen. Aber aus Sicht des Unternehmens ist das nicht unbedingt 3 effizient.

...two things were significant for the later development. I had been on the move internationally a lot and that is why I could not, as one says, take root somewhere... For me there really just was the job and then at the weekend family. I had no hobbies, for hobbies I had no time. Since during the week my job was really open end, I kept the weekend free deliberately to spend time with the children ... That meant in consequence, I had no big network outside of my job and I also didn't have some memberships in clubs or hobbies such as tennis or golf or whatever. Where you could say there are a few people, who will also be there, when you have a lot of time as is the case now. $[\ldots]$

LB: Sag mal, was hat dir denn, wenn du so zurück guckst, was hat dir am meisten Spaß gemacht bei deinem Beruf und was hat dir am wenigsten Freude bereitet?

...when you look back, what did you enjoy the most and the least in your career?

7 GB: Also, pass auf, am meisten Spaß hat mir gemacht das Reisen und die 8 Entscheidungsfragen. Ich habe immer gesagt, meine beste Zeit, meine glücklichste Zeit war 9 die als Zentraleinkäufer, weil da war niemand, der mir in irgendeiner Form Vorschriften 10 gemacht hat solange ich erfolgreich war in dem was ich tat und entschieden habe, hatte ich 11 alle Freiheiten dieser Welt. Wenn ich gesagt habe, ich muss zwei Wochen nach Asien fahren 12 und einkaufen, dann fuhr ich zwei Wochen nach Asien. So. Und wenn ich gesagt habe, der 13 Lieferant ist besser als der, dann hat man es mir geglaubt, weil ich war der Fachmann. So. 14 Also, die größte Freiheit hatte ich als Zentraleinkäufer. Das meiste Geld habe ich natürlich 15 als Topmanager verdient, nur du spielst dann in einer ganz anderen Liga. Dann geht es um 16 Politik, um Diplomatie, um Abstimmungen, um Abwägungen, das ist dann, sage ich mal, 17 manchmal mühsam, weil du eigentlich kaum noch was selber entscheiden kannst. Du musst 18 immer abstimmen, es muss, ja so-. Und du hast auch dann eine ganz andere Art von 19 Verantwortung. Das hat mich aber nicht gestört, im Gegenteil. Ich habe da die 20 Verantwortung als Führungskraft für Menschen, das hat mir immer, Spaß ist jetzt der falsche 21 Ausdruck, da war ich mir immer ganz besonderer Verantwortung bewusst. So. Ich wäre 22 vielleicht weitergekommen, wenn ich manchmal über Leichen gegangen wäre. Das habe ich 23 nie getan. Letztendlich war für mich immer noch der Mensch wichtiger als der Job. Und ich 24 konnte nie, sagen wir mal, jemandem besser in den Rücken stechen, damit ich davon, weil 
ich davon einen persönlichen Vorteil gehabt hätte. Das ging nicht. Ich habe allerdings darunter ein Stück weit gelitten, wenn ich feststellte, dass Kollegen oder auch Vorgesetzte, sage ich mal, sich auf meine Kosten profiliert haben. Mir hat mal mein Chef als ich in Hong Kong CEO war, hat der zu mir gesagt: „Herr B.,“ ein halbes Jahr war ich gerade da, also relativ frisch, ,ich höre aus der Organisation, dass Sie zu weich sind in Ihrer Führung. Die Metro braucht harte Führer.“ So. Dann habe ich gesagt: „Herr Wolfram,“ ich war ja nun- ich kam ja aus zwei Jahren Direktor Kaufhalle und habe dann gesagt: „Herr Wolfram, bisher hat mir noch niemand bewiesen, dass er meine Fairness missbraucht." Ich hatte nicht das Gefühl,

9 dass ich morgens jedem erst mal zwischen die Augen schlagen muss, damit er zu arbeiten 10 fängt. So. Und das war so eine Art Grundverständnis für mich von Führung. Was nicht heißt, 11 dass ich nicht Leute, die sagen wir mal, irgendwo Mist gebaut haben, nicht kritisieren konnte. Ich hatte ja keine Angst vor Kritikgesprächen, aber ich habe Menschen, ja, immer als Mensch gesehen und nicht als, sagen wir mal, Instrument der Firmenpolitik.

Well, listen, I enjoyed travelling and issues which involved making decisions. I have always said my best time; my happiest time was when I was central purchaser since there was nobody who in any way lay down the law to me as long as I was successful in what I was doing and had decided, I had all the freedom of the world...I, of course, earned the most money as a top manager, only that then you play in totally different league. There it all revolves around politics, diplomacy, coordination, evaluating and that is then, let's say, sometimes tiring since you can hardly decide anything by yourself....I could have perhaps reached more if I had sometimes sold my own grandmother. I never did that. In the end, the human being was always more important to me than the job.

14 LB: Aussterbend, befürchte ich fast. Oder hast du den Eindruck, dass es da noch sehr viele

15 Firmen gibt, wo das eine Rolle spielt?

Dying out, I'm afraid. Or do you have the feeling that there are still many companies where this plays a role?

16 GB: Nein, das glaube ich nicht. Aber das, daran kannst du eigentlich so ein bisschen den

17 Niedergang unseres Systems festmachen. Weil, wenn ich also noch so ein paar

18 Unternehmerpersönlichkeiten sehe, wie so ein Deichmann beispielsweise, ja oder andere.

19 Die sterben ja alle aus. Aber es sind noch ein paar Unternehmerpersönlichkeiten, die sagen,

20 wie der Beisheim, der Otto Beisheim hat immer gesagt, ich meine, der war mal der reichste

21 Mann Deutschlands so ungefähr mit zehn Milliarden Privatvermögen, der war ja auch in

22 der Schweiz immer wenn es Verwaltungsratssitzungen gab, da hat er zu mir gesagt: „, Wissen 
Sie Herr B., wenn ich abends mein Leberwurstbrot gegessen habe, bin ich auch satt“. Ja. Das sind Leute mit der Einstellung, sage ich mal, für die ist das nicht kriegsentscheidend, ob da jetzt jedes Jahr eine Milliarde Gewinn noch dazukommt. Die- wenn die satt sind, dann sind die satt. So. Und da-dieses Verständnis, sage ich jetzt mal, dass man als Topmanager wirklich auch eine Verantwortung für die Menschen hat, die vom Unternehmen abhängen, die ist ganz weit abhandengekommen, weil die werden ja alle nur noch beurteilt durch die Analysten. Wenn es- wenn es Kapitalgesellschaften sind, so, die werden nur noch beurteilt von den Anteilseignern, nach sagen wir mal, ihrem Ebit Zuwächsen, wie die Kurse sich entwickeln und den Shareholdern ist es dann auf Deutsch gesagt, scheißegal, Hauptsache, er kriegt mehr Dividende. So. Und wenn ich dann gestern gehört habe, dass der Herr Quandt so ungefähr jedes Jahr eine Milliarde Dividende von BMW kriegt, dann frage ich mich, sage ich mal, was- wohin soll das führen. Wo, wo ist eigentlich dann für diese Leute das Verständnis, dass ihre Aufgabe im Leben eigentlich nicht ist, ins Grab zu gehen mit eher zehn Milliarden Vermögen als acht Milliarden, sondern, dass das Ziel eigentlich sein muss, was zu hinterlassen was ein bisschen, sagen wir mal, nachhaltiger ist als die Frage, zehn oder acht Milliarden. Und- ich kann das auch festmachen an der eigenen Erfahrung. Ich habe - das hat mich auch ... Heute, seine Tochter ist jetzt noch in der Metro, so. Und die sagt mir manchmal, weißt du, ich habe den und den wieder getroffen, die schwärmen heute noch alle von dir. Und nicht- kein Mensch von denen weiß mit wieviel Umsatz ich die Metro verlassen habe. Wieviel Länder ich aufgemacht habe in meiner Eigenschaft als Generalbevollmächtigter für Expansionen. Das weiß kein Mensch. Aber, dass ich jemand war, der offen und fair war, wo Leute gerne mit gearbeitet haben, wo sie nicht den Eindruck hatten, dass nur noch, sagen wir mal, gedrängelt wird und geprügelt, oder sagen wir mal, falsch beschuldigt wird, dass war das, wofür ich eigentlich gestanden habe. Ich bin überzeugt, ich hätte noch einen Stern mehr auf die Schulter kriegen können, wenn ich, sage ich mal, in Anführungsstrichen , eine Sau“ gewesen wäre, ja und mich auf Kosten anderer noch versucht hätte zu profilieren. Das war mir das nicht wert. Das war mir das nicht wert. Also, ich wollte immer morgens in den Spiegel gucken und vor mir selber dann auch sagen können, ich kann meinen Leuten, mit denen ich gearbeitet habe, immer gerade in die Augen gucken.

...But that, this exemplifies the decline of our system. Because, when I, well, see some of these entrepreneurs ...they all die out... Otto Beisheim always said, I mean he was once the richest man in Germany with roughly 10 billion private assets...he was also in Switzerland ... and he had said to me: 'You know Mr B when I have eaten my bread with liver sausage I am also full.' Yes, these are people with the attitude, let's say, for them, it is not decisive for 
the war if one billion of profit is added every year. They, when they are full, they are full. So. And that...this view, let's say, that as a top manager you really also have a responsibility for the people who are dependent on the company, that very much got lost since they all are evaluated by the analysts. When, when it is a limited liability company, so, they are only evaluated by the shareholders, by, let's say the EBIT increases, by how the share prices developed and the shareholders, in plain German, don't give a damn. The main thing is that they receive a higher dividend. And when I then yesterday heard that Mr Quandt roughly receives one billion dividends from BMW, then I ask myself, let's say, what, where shall that lead. Where, where is the understanding of these people that it is not their job in life to take rather ten than eight billion with them in the grave, that the real aim is to leave behind something which is a little, let's say, more sustainable than ten or eight billion. ... nobody of these people knows with how much turnover I had left the Metro, how many countries I established in my job as a fully authorised representative. Nobody knows that. But that I was somebody who was open and fair, whom people liked working with, with whom they didn't get the impression, that, let's say, that they were only pressured and beat, or let's say, wrongly accused, that was really what I stood for. I am convinced that I could have climbed the career ladder higher, had I, let's say, been an 'ass', well and had tried to profit at someone else's expense. That wasn't worth it for me. That wasn't worth it for me. Well, I always wanted to look at myself in the mirror in the morning and be able to tell myself that I could look the people I worked with straight in the eye.

1 LB: Ja, das halte ich für ganz wichtig. Und auch dass du sozusagen das repräsentierst, was

2 du auch von denen, die für dich arbeiten, erwartest. Ja. Weißt du, das ist eben - das kenne

3 ich ja auch aus meiner eigenen kleinen Erfahrung. Wenn die Leute, die über dir stehen, das

4 nicht repräsentieren, was du dir erwartest, dann kannst du die auch nicht respektieren. Das

5 geht einfach nicht. Also, sehr, sehr schwierig. Hatte denn der Beruf an sich, denkst du, eine

6 Auswirkung darauf, wie du dich gesehen hast? Wie du dich als Mensch definiert hast?

...What do you think, has your job had an influence on how you viewed yourself? How you define yourself as a human?

7 GB: Ja. Also, wenn ich das heute rückwärts betrachte - ich war - bin viel weitergekommen, 8 als ich eigentlich als junger Mann im Handel für mich selber als Ziel gesteckt hatte. Mein 9 Ziel als ich angefangen habe in der Kaufhalle mit einem weißen Kittel auf der Sülzburgstraße 10 mit Bananen und sah die Zentraleinkäufer, ja, wenn die mal in die Filiale kamen, ja, das war 11 mein größtes Ziel, Zentraleinkäufer zu werden. So. Das hatte ich ja nun relativ schnell 12 erreicht. Und ich bin 1973 Zentraleinkäufer geworden, das heißt also ungefähr vier Jahre, 
nachdem ich in der Kaufhalle angefangen habe. Und das war schon super. Und da- So. Alles, das danach kam, sage ich mal, war von mir nicht geplant und auch nicht als Ziel gesetzt. Ich habe dann allerdings im späteren Verlauf, deshalb habe ich auch gesagt, die schönste Zeit war eigentlich die Zentraleinkäuferzeit, weil, was dann danach kam, war oft mit Kompromissen dann gespickt, mit auch mal eine Faust in der Tasche machen, ja und gerade dieser bewusste Herr Röschmann, der mein Chef über viele Jahre war, als ich in Hong Kong war, war er mein Chef und als ich in der Schweiz war bis 1996 war das auch da mein Chef, weil der war im Vorstand der Metro International. Und mit dem hatte ich ein Riesenproblem, weil der mit mir ein Riesenproblem hatte. Da musste ich dann auch eine Faust in der Tasche machen, was mir eigentlich so nicht gut bekommen ist. Aber ich habe das nie irgendwie nach unten weitergegeben, weil das betraf in erster Linie mich. Und ich wollte nie, dass meine Mitarbeiter da in irgendeiner Form tangiert werden. Das waren so Profilierungskämpfe, wo der dann versucht hat, mich schlecht zu machen. Das war ein Manager, das - der seine eigene Sicherheit damit gesehen hat, dass die Leute, die er in seinem Gesprenge hatte, so mit Angst und Zweifel zu führen, dass die so ungefähr ihm gegenüber dankbar sein mussten, dass sie überhaupt noch da sein durften. So. Das verschaffte ihm dann selber, sagen wir mal, Sicherheit in seiner Position, so dass keiner an seinem Stuhl sägen konnte. Und machte ihn so ein bisschen unangreifbar und king of the kings. Das ging mir natürlich extrem gegen den Strich. Weil, also ich konnte mich nicht verbiegen, und ich war auch nie der Typ, der sein Rückgrat an der Garderobe abgegeben hat morgens. Und deshalb hatte ich dann auch oft mit ihm Konflikte, weil ich in der Sache, in der Materie auch besser Bescheid wusste als er. Und das passte ihm natürlich dann überhaupt nicht. Denn er musste als Chef immer besser wissen, worum es ging. Und, das war nie mein Verständnis. Ich habe oft als... In der höchsten Position, die ich hatte, als Generalbevollmächtigter, ich meine Corporate Development war was völlig Neues in der Metro, das habe ich aufgebaut, die Abteilung gab es vorher überhaupt nicht. Und da ich selber nicht über das Wissen verfügte, habe ich mir ein paar junge Leute geholt, die also Corporate Development, sage ich mal und andere Dinge, sagen wir mal, an der Uni gelernt hatten. So und das gepaart mit meinem, sagen wir mal, Heringsbändiger-Erfahrungs-Praxis-Wissen, war in der Mischung natürlich goldrichtig. Und...ich habe aber meine jungen Leute, wo immer es ging, präsentiert und habe die im Vorstand dann präsentieren lassen. Ich habe nie gesagt, ich muss selber präsentieren, damit der Vorstand dann sagt, hat der B. eine tolle Präsentation gemacht. Nein. Meine Freude war, wenn ich einen von meinen jungen Leuten die eine Präsentation erarbeitet hatte oder Visibility Studien für neue Länder. Dann waren die mal drei - vier Wochen da. Ich war fünf Tage da und die vier Wochen und habe das alles aufbereitet. Dann habe ich die dem Vorstand das präsentieren lassen. Weil ich einfach froh war, dass ich einem Jungen eine Chance geben 
1 konnte mal in so einem Auditorium vom Vorstand präsentieren zu können. So in dem

2 Verständnis habe ich dann auch... Ich habe ja fünf Jahre in St. Gallen als Coach die ersten

3 zwei Semester begleitet. Also dann hatte ich immer, in jedem Jahrgang, zwei so junge

4 Studenten und das hatte St. Gallen als eine der wenigen Universitäten. Das waren

5 europäische, also von der EU ein Modell, wo also in verschiedenen Ländern die

6 Universitäten dieses Coaching-Modell aufgesetzt haben, um gerade den ersten und zweiten

7 Semestern neben der Wissensvermittlung, die an der Uni passiert, so ein bisschen auch in

8 der Menschenführung, in der Persönlichkeitsentwicklung ein bisschen, sagen wir mal,

9 Hilfestellung geben konnten. Und das war dieses Coachingsystem. Und das habe ich

10 unheimlich gerne gemacht. Ich habe dann auch meine Vorurteile der jungen Generation

11 gegenüber sofort komplett revidiert. Also, null Bock Generation war falsch. Ziehe ich

12 zurück, weil ich junge Leute kennengelernt habe, die mit einem unheimlichen Engagement

13 und Begeisterung und Motivation dann an die Uni gekommen sind. Und da St. Gallen ja

14 sehr, sehr stark international besucht war.

I then also immediately completely revised my prejudices against the young generation. So, the 'couldn't be buggered-generation' was wrong. I take it back since I got to know young people who came to university with an incredible degree of commitment and enthusiasm and motivation.

15 LB: Ja, das sagt mir durchaus was.

Yes, I have heard about this.

16 GB: ... da waren viele junge Leute und für die ist das ja einen Riesenchange gewesen. Die 17 sind das erste Mal von der Familie weg, kommen in ein neues Land, kommen an eine Uni, 18 wo es nicht wie in der Schule einen festen Stundenplan gibt von der ersten bis zur sechsten 19 Stunde, und die sich selber organisieren müssen. Gepaart mit wohnungsmäßig neu 20 organisieren, in WGs oder bei Senioren. So. Und dann sind die Eltern weit weg, die Freunde 21 weit weg und gut, dann war der B. da, wo sie sich immer mit unterhalten konnten, wo sie 22 auch mal Probleme mit diskutieren konnten, die sie vielleicht sogar mit ihren eigenen Eltern 23 nicht diskutierten wollten oder was. Und das hat mir unheimlich viel Freude gemacht.

...So. And then the parents are far away, the friends are far away and, well, then there was B., who they could always talk to, who they could discuss problems with which they possibly didn't want to discuss with their parents or something like that. And that I enjoyed immensely... 
LB: Ja, Mentor - das hast du so ein bisschen...

Yes, mentor - you have a bit of that...

2 GB: Ja, Mentor.

Yes, mentor.

3 LB: Also, ich kann mir das sehr gut vorstellen, weil du interessiert bist und das ist sehr selten 4 der Fall.

Well, I can imagine that very well since you are interested and that is seldomly the case.

5 GB: Ja, das hat mich auch interessiert und ich habe- Ich meine, das ist ja- Der Professor von

6 der HSE, der das gemacht hat, der hat immer gesagt: „Herr B., also wir haben ein Problem“.

7 Es gab 40 Coaches in dem Programm und die sind ja alle auch mit ihrem CV dann auch an

8 dem System der Uni hinterlegt und die jungen Studenten, die kamen, die konnten sich dann

9 aussuchen, wen sie in Priorität eigentlich in erster, zweiter Stelle gerne als Coach hätten.

10 Und der sagte dann immer: „Das Problem, das wir haben, es gibt mittlerweile bei uns eine

11 B.-Skala, weil Sie immer mit Abstand der sind, der die meisten first choices hat. " Und ich

12 meine, das spricht sich ja rum. Die jungen Studenten, die kommen, die sprechen natürlich

13 mit ihrem Vorgänger und sagen, hör mal, kannst du mir einen empfehlen, wer ist denn gut?

14 So. Und da hatte ich halt eben einen guten Namen. Ich hatte natürlich auch den Vorteil, dass

15 ich, ich glaube sogar der einzige von den Coaches war, der selber nicht mehr gearbeitet hat.

The professor of the HSE...always said...:'The problem we have is that by now we have a B-scale since you are by far always the one with most first choices'...

$16[\ldots]$

17 LB: Ja, völlig. Da wäre ich auch völlig begeistert gewesen.

...I would also have been totally thrilled.

18 GB: Ich habe immer gesagt, dem Professor gesagt: „, Sie müssen mir nicht danken. Ich muss

19 eigentlich Ihnen danken. Ich habe so viel gelernt von den jungen Leuten. “ Ja, ich meine,

20 wenn ich denen was gebracht habe, ist es noch so schön. Wenn die irgendwann später als

21 Erwachsene und Topmanager sagen, also in meinen Studentenjahren habe ich so einen alten

22 Guru aus dem Handel (Türklingel schellt und Hund bellt) gehabt, dann ist das super. Dann

23 ist das alles super.Und ich glaube, dass da auch vieles bei ist, was wirklich aber Bestand hat 
nach vorne. Das verändert sich nicht. Da hat auch nichts mit, was weiß ich jetzt,

2 Digitalisierung $\mathrm{zu}$ tun. Das gilt für Prozesse, aber nicht für Grunddinge im

3 Zusammenwirken. Und für mich waren ja immer so wichtig, bestimmte Werte. Also die

4 Werteorientierung sage ich mal. Du musst keine Sau sein, um erfolgreich im Job zu sein.

I always said, told the professor: 'You don't have to thank me. Really I would have to thank you. I learned a lot from the young people.' Yes, well, I think, if I provided them with something, it is nice. When they at some point later as adults and top managers say, well during my studies, I was taught by an old guru of purchase, then that is great.

6 LB: Ja, weil das so eine Dynamik abkriegt.

Yes, since it becomes a dynamic process.

7 GB: Ich sage dir ein Beispiel. Ich war mal bei einem Arzt. Und dann hat der gesagt, Herr B. 8 wir machen ein Langzeit-EKG. Und ich war jemand, der sein Leben lang mit sehr niedrigem 9 Blutdruck hervorragend überall durch die Bache ging. Das war für mich wie eine 10 Lebensversicherung. War ich auch sehr dankbar für. Bis zu dem Zeitpunkt, wo der mit dem 11 Langzeit-EKG festgestellt hat, dass ich nachts bis zu 200 Blutdruck hatte. Und da hat er 12 gesagt, Herr B. kann es sein, dass sie Stress so runterfahren oder verdrängen, dass sie in der 13 Nacht im Schlaf anfangen ihren Stress abzuarbeiten. Da habe ich gesagt, ich schlafe 14 traumlos. Aber ich bin selber erschrocken, dass ich also offensichtlich in völlig chaotischen, 15 hohen Blutdruck in der Nacht hatte, im Schlaf. Und dann kam aber irgendwann mit 59, der erste Stent. Und bei dieser Untersuchung hier an der Uniklinik stellte der Professor Erdmann fest, sagt der och, gucken sie mal. Das kannst du ja mit im Monitor gucken. Wenn der mit dem Herzkatheter zugange ist. Sagte, wissen sie, dass sie einen Hinterwandinfarkt hatten?

19 Nein, sage ich. Habe ich nichts von gemerkt. Hat er gesagt, normalerweise stirbt jemand 20 daran. An dem Hinterwandinfarkt. Sagte, gucken Sie mal. Ich komme mit dem Katheter 21 überhaupt nicht mehr dadurch. Das ist wie Beton. Ich sage, und warum ist nichts passiert? 22 Ja sagt er, gucken sie mal da. Ihr Herz hat natürliche Bypässe gebildet. So, dass die Arterie, die in die Hinterwand ging ist $\mathrm{zu}$, wie Beton. Aber es gab um das Herz darum natürliche Bypässe und mein Herz hatte die gleiche Pumpleistung wie vorher. Nichts, keine Beeinträchtigung, gar nichts. Sagt er, habe ich noch nie gesehen. Ist ein Phänomen. Sagte er, können sie dankbar sein. Aber das Ding was ich hier jetzt gekriegt habe, den Stent, der war auch nötig. Das war fünf vor zwölf. Und da habe ich gedacht, ich habe geraucht mein ganzes Leben lang. Aber nie getrunken, also keinen Alkohol. Habe allerdings auch nicht viel Sport 
gemacht. Und das war dann die Wirkung, Wende, sage ich mal. Wenn du einen Job hast, der dich... was da gemacht wurde, unregelmäßiges Essen, falsches Essen, keinen Ausgleich. Und das ist die Langzeitwirkung. Und das habe ich begriffen, eigentlich erst sehr, sehr spät. Im Alter. Dass die Psyche, oder was man in der chinesischen Medizin Yin Yang nennt. Dass das ganz, ganz wichtig ist. Wenn du das nicht beachtest, dann kriegst du, sage ich mal, über die Zeit Probleme an Ecken, die du gar nicht im Vorhinein definieren kannst. Weil du nicht weißt, wie dein Körper langfristig an welcher Ecke reagiert. Aber für mich ist das heute völlig klar. Und deshalb-. Ob ich heute nochmal den gleichen beruflichen Weg gehen würde, dass weiß ich nicht. Ich habe oft überlegt, wenn ich Anwalt geworden wäre. Also ich habe ein extremes Gerechtigkeitsbewusstsein. Das hätte auch sein können, dass ich also psychisch daran zugrunde gegangen wäre wenn ich, sage mal, Unschuldige nicht hätte erfolgreich verteidigen können und Schuldige hätte frei-verteidigen müssen. Also da kannst du auch Probleme mit dir selber kriegen. Aber so erfolgreich, wie ich auch war, in dem was ich getan habe. Ich habe auch dafür auf vieles verzichten müssen. Also was Familie anbelangt, das hat-. Guck mal, die Tatsache, dass ich mich von der Brigitte, dass wir uns getrennt haben-. Das war, sage ich mal, ich habe das immer so ein bisschen burschikos gesagt. Ich habe meine erste Ehe auf dem Altar meiner Karriere geopfert. Die Brigitte war an der PH und war damals, sage ich mal, so ein bisschen in dieser grünen Ecke. Das war ja damals die Großzeit der APO, die späten sechziger Jahre. Und ich war in einem erzkonservativen, kapitalistischen orientierten Unternehmen und wollte Karriere machen weil ich wollte nicht im Kittel bleiben. So. Und das fing damals schon an, sage ich mal, sich nicht zu vertragen. Weil die Brigitte so ein bisschen, wie auch die Gabi, extremer, die Susanne sehr dezent mit ihrem Vater das Problem hatten, dass sie durch seine Karriere und durch seine Dominanz viel zurückstecken mussten. Er war der Primus inter pares und der absolute Lenker in allem. Und das fanden die Töchter nicht so toll. Und ich hatte immer gedacht, also wenn es einen gibt, der Verständnis haben muss, dass ich Karriere machen will, dann ist es die Brigitte. Die hat aber eigentlich dann sehr wenig Verständnis, weil sie genau das bei ihrem Vater eigentlich auch nicht akzeptiert hatte. Und das ging dann auf Dauer nicht gut. Heute würde ich mir das überlegen. Wobei auch offen sagen muss, in dem Alter, ob du da schon mit so viel Abgeklärtheit und Weitsicht überhaupt entscheiden kannst, was das richtige für dich ist, da kannst du auch ein großes Fragezeichen machen.

...When you have a job... and what was done there, irregular meals, wrong food, no balance. And that is the long-term effect. And that I only understood, very, very late. In older age. That the psyche, or what you in the Chinese medicine call Yin Yang, that that is very, very important. If you don't take care of this, you will in the long run get problems in regions you 
cannot determine beforehand. Because you don't know how your body will react in the long run and in which area. But for me this totally obvious today...As successful as I was with what I was doing, I also had to sacrifice a lot for that. Well, regarding my family, that was ...You see, the fact that I separated from B., that we separated ...That was, let's say, I used to say this quite tomboyish: I sacrificed my first marriage on the altar of my career. ... Today I would think about this.

$[\ldots]$

2 LB: Hast Du denn grundsätzlich das Gefühl, das junge Leute dich aufgrund deines Alters 3 anders behandeln, anders sehen als gleichaltrige oder jüngere?

Do you generally have the feeling that young people treat, view you differently to people their age or younger?

4 GB: Doch. Aber ich hatte den Eindruck, dass die alle extrem überrascht waren, wie ich auf 5 sie eingegangen bin, wie ich mit ihnen umgegangen bin. Ich habe immer mit denen auf 6 Augenhöhe gesprochen. Und die haben auch gemerkt, dass ich das toll fand mit einem 7 jungen Menschen zu reden und einfach nachzuvollziehen, wie der so tickt und was der will 8 und macht. Und ich habe auch ganz klar das Gefühl gehabt, die haben verstanden, dass ich 9 jetzt nicht komme nach dem Motto-, das habe ich vor 40 oder 50 Jahren alles selber schon 10 erlebt, das kannst du vergessen. Sondern mit dem ehrlichen Bedürfnis, sage ich mal, dass 11 was ich aus dem Verständnis, was der junge Mensch will mit meiner Erfahrung ebenso ein 12 bisschen auf Tellerminen aufmerksam machen kann, die irgendwo im Gelände liegen 13 könnten. Aber ihm nicht den Schneid abkaufen will, sondern im Gegenteil. Ihn motivieren 14 will, seine eigentliche Stärke zu erkennen und auch dazu zu stehen und das fest zu machen. 15 Ich habe da das so oft im Leben erlebt. Leute, ob in Präsentationen, in Vorträgen. Ich habe 16 vor fünfzehnhundert Leuten in der Messehalle in Düsseldorf moderiert, den ganzen Tag, 17 eine Lieferantenkonferenz, Du kannst nicht wie ein Schauspieler den ganzen Tag oder über 18 Dauer Dinge vertreten, die du nicht von innen trägst. Diese Authentizität muss da sein. Und 19 die muss auch rüberkommen. Dann springt der Funke. Und dann kann jemand sagen, ich 20 sehe das ganz anders. Aber er kann nicht sagen, der spielt was, was er nicht ist.

Yes. But I had the feeling that they were all extremely surprised how I responded to them, how I treated them. I always talked on eye level with them. And they also noticed that they found it great to talk to a young person and to understand how he ticks and what he wants to do. And I also really had the feeling that they understood that I didn't go there with the intention: I experienced all that 40 or 50 myself, you can forget that. But with the honest 
intention, let's say, that with my insight into what the young person wants to achieve, I can guide their attention towards any land mines that might be placed somewhere. But that I didn't want to dishearten him but the contrary. I wanted to motivate him to discover his own strength and to stick by it and to demonstrate that. Authenticity has to be there... Somebody can say I view this differently, but he cannot say, he pretends to be something what he isn't.

$1 \quad[\ldots]$

2 LB: Aber wie war das denn für dich dann? Also bist dann aufgewacht und dann warst Du 3 Rentner. Wie war das?

And how was that for you? So you woke up and then you were a pensioner: how was that?

4 GB: Also es war eigentlich traumatisch, sage ich jetzt mal. Weil du kommst dir plötzlich vor, 5 als wärst du völlig überflüssig. Also das ist heute noch eigentlich so. Wobei das jetzt in der

6 Zeitachse, bei mir jetzt sechs Jahre bin ich offiziell pensioniert. War das temporär natürlich 7 durch meine Krankheit anders in der Priorisierung. Aber es ist heute noch so, dass ich 8 eigentlich sage, es ist eigentlich schade um die Zeit, die ich lebe ohne sie mit etwas füllen zu 9 können, von dem ich selber sage, es macht Sinn. Und jetzt gibt es Leute, die mir gesagt 10 haben, Mensch G., du hast 42 Jahre hart gearbeitet. Es steht dir zu und es ist eigentlich 11 normal, dass du jetzt nichts tust. Nein. Das ist für mich eben nicht normal. Jetzt kannst du 12 sagen. Okay, dann tu etwas ehrenamtlich. Also es gibt, was weiß ich, jetzt Businessangels 13 oder wie sie alle heißen. Die Plattformen. Da habe ich mal reingeguckt. Da sind also 14 irgendwie 3000 Leute, die sich mit ihren CVs da als Berater anbieten. Das ist nicht meine 15 Sache. Dann habe ich überlegt, ich wollte mal eine Stiftung gründen. Da hatte ich aber dann 16 nicht das Geld zu, das zu machen. Ich wollte in jedem Fall etwas für, sage ich mal, Kinder 17 tun. Schon irgendwo mit dem Gedanken, was kannst du denn mit deiner Zeit jetzt anfangen. 18 Aber das dominante Gefühl ist eigentlich-. Also gestern, sage ich mal, hattest du einen riesen 19 Sack von Terminen und Aufgaben und warst ganz wichtig. Und heute interessiert das 20 eigentlich keine Sau. Was du machst und wie du es machst. So. Und-.

Well, really, that was traumatic, let's say. Because you suddenly feel as if you were totally dispensable. ... That is the case till today so that I really say; it is a waste of the time I live without being able to fill it with something, I could say it makes sense...Well, yesterday, let's say you had a bag full of appointments and tasks to be done ${ }^{28}$, and you

28 ,bag full of appointments' is a colloquial expression for having a packed agenda 
were very important. And today nobody really gives a damn. What you do and how you do it...

1 LB: Hat also schon Einfluss wirklich auf deine Identität? Auf dein Gefühl, was dich selber 2 angeht?

So it really has an effect on your identity? On your feeling with reference to yourself?

3 GB: Ja, ganz klar. Aber sagen wir soweit, dass-, und das ist vielleicht jetzt meine Mentalität, 4 weil ich relativ stark auch selber reflektiere. Dass du ein Problem damit hast irgendwas zu 5 tun. Weil ich mir dann sage würde jetzt nicht etwas tun, wo ich nur noch ein Alibi habe. 6 Nach dem Motto, Hauptsache ich bin von der Straße weg, das ich nicht unter das Auto $7 \quad$ komme. Das reicht mir nicht. Also es müsste schon etwas sein, was mich auch mit Sinn 8 erfüllt. Wo ich sage, ich kann etwas bewegen, ich kann etwas tun. Deshalb das Thema, mit 9 Studenten oder jungen Leuten oder wenn diese Firma gesagt hätte, pass auf B., wir machen, 10 was weiß ich, im Abstand von x machen wir immer Führungskräftenachwuchsseminare. Und 11 da kannst du immer etwas über Einkommen erzählen, oder über Changemanagement oder 12 Internationalisierung. Ja, was bedeutet es, wenn ich in einem anderen Land etwas machen 13 will. Ja, wo muss ich drauf aufpassen und solche Dinge. Das hätte ich gerne gemacht. Auch 14 ohne Geld. Wenn es mir eher nur darum gegangen wäre, etwas weiterzugeben, was 15 gebraucht wird. Was Sinn macht. Ja? Tamara sagt du musst ein Buch schreiben. Weiß ich 16 nicht.

Yes, definitely....That you have a problem to do just something. Because I would tell myself, don't now do something just to have an alibi. According to the principle, the main thing is that he is off the road so that I don't get run over by a car. That is not enough for me. I would have to do something which fills me with sense. Where I can say, I can move something, I can do something....For me, it would have been more significant to pass on something which is needed. What makes sense. Yes? T. says I should write a book. I don’t know.

17 LB: Aber die Familie kann das für sich auch nicht-.

But the family can also not -.

18 GB: Nein. Also sagen wir mal so. Wie tief das geht, kannst du daran festmachen. Ich bin ein 19 sehr gläubiger Mensch. Also kein Katholik, im Sinne der katholischen Kirche, Gesetze. Aber 20 ich bin ein gläubiger Mensch. Und seit ich meinen Lungenkrebs ja überwunden habe, habe 21 ich mich oft gefragt. Also der Herrgott. Musst Du wissen, mein Bruder, der ein Jahr älter 22 war, ist da mit 55 Jahren dran gestorben, an Lungenkrebs. Und hat drei Jahre, zwei oder drei 
Schädeloperationen gehabt. Metastasen am Kopf. Der ist ganz schlimm gestorben. Das hat mich unheimlich auch aus der Kurve geschmissen. So, jetzt hatte ich das selber. Ich weiß nicht warum. Ich habe irgendwie gesagt, daran sterbe ich nicht. Bin auch nicht daran gestorben, obwohl ich hatte einen massiven Tumor, sieben Zentimeter Tumor auf der Lunge hatte. Drei Zentimeter Metastase im Kopf. Also ich sitze noch da. Dann habe ich gesagt, Herrgott, du musst dir ja etwas dabei gedacht haben. So. Und jetzt gucke ich jeden Tag wirklich links und rechts, sehr aufmerksam, dass ich das finde, wofür der Herrgott mich aufgehoben hat. Jetzt kannst du sagen, das ist ja völlig spinnert, für jemanden, der Generalbevollmächtigter bei der Metro war. Einen riesen Job. Aber es ist so ein bisschen kennzeichnend für die Suche auf die du dich begibst. Das ist dann-, dann kannst du auch sagen, ich suche meine neue Mitte. Früher war meine Mitte der Job und die Familie. In der Kombination. Und jetzt ist die weg. Für meine Frau, für die Tamara beispielsweise, war es enorm schwierig, heute noch. Von jetzt auf gleich beispielsweise, waren die Kinder aus dem Haus. Das war für sie eigentlich der Lebensinhalt. Und sie hat mir dreißig Jahre das Kreuz freigehalten. Das heißt also alles, was Familie war, was Kinder war, war ihr Job. So. Und jetzt waren die Kinder plötzlich aus dem Haus, ich hatte den Job nicht mehr, das große Haus hatten wir auch nicht mehr. Sie war aber dreißig Jahre aus dem Job raus. Und jetzt sucht sie eigentlich wieder einen neuen Beginn. So ein bisschen beruflich. Und ich habe immer gesagt, weißt du, was ich tun kann. Sie ist 53 also 54 gerade geworden. Ich bin 71. Ich bin Rentner. Du, wenn ich daran denke, wie ich mit 54 gedacht habe, dann bist du meilenweit davon weg. Und ich würde um Gottes Willen nicht von dir erwarten, dass du jetzt so lebst, wie ich meine mit 71 leben zu können oder zu müssen. Ich helfe dir in allen Fällen, was du tun willst, um dich zu unterstützen. So. Das ist so. Wirklich jetzt immer noch, auch nach sechs Jahren. Die neue Mitte zu finden. Das, was dich trägt, was dich ausfüllt. Das ist so eine Gemengelage, die einfach nicht klar ist.

No. Let me say it this way. How deep that goes, you can see. I am a believer. Not a catholic, in how the church prescribes it, but I am a believer. And since I managed to get over my lung cancer, I often asked myself ... Then I said, God, you must have had a plan. So. And now I really look right and left, very attentively, so that I can identify what God has saved me for. ...But that is somewhat characteristic for the search you are conducting. That is then, ...you then could also say, I am looking for a new centre of my life. Before that, were my family and the job. In this combination. And now that is gone.... That therefore means, everything which was family, what had to do with the children was her job. 
Do you feel old?

1 GB: Nein, als alt empfinde ich mich überhaupt nicht. Ich wundere mich manchmal-, also

2 ich sage manchmal so ein paar Zellen, sage ich mal, sind bei der Bestrahlung

3 mitkaputtgegangen, aber ansonsten, sage ich mal, habe ich noch die sieben Sinne

4 beisammen. Auch körperlich. Ich meine, ich bin jeden Tag zwei Stunden mit dem Hund

5 unterwegs, an der frischen Luft. Das bekommt mir sehr gut. Nein, ich habe auch keine

6 Probleme mit-, ich habe auch nicht jetzt Demenz oder sonstige Probleme. Das einzige

7 Problem, was ich habe ist, dass ich also mit meiner Kraft, meiner geistigen und körperlichen

8 Kraft mich völlig nicht nur unterfordert, sondern mich überhaupt nicht irgendwo platziert

9 fühle. Weißt Du? Ich habe mal so ein bisschen nonchalant gesagt, ich stehle dem Herrgott

10 wertvolle Zeit. Insofern, dass ich eigentlich mehr tun können müsste und auch wollte, als ich

11 de facto tue.

...well, I sometimes say that some brain cells, let's say, were destroyed during chemotherapy but besides that, let's say, I am in full command of my senses. Also physically... No I have no problems with, ...I don't have dementia or something like that... The only problem I have is that I, therefore, with my power, my mental and physical power not only feel totally underchallenged but that I do not at all feel placed somewhere. You know? I once said, rather colloquially, I steal God valuable time. As such as that I could really do more and would also like to do more than I'm factually doing.

$13 \quad$ LB: ... Setzt Du dich mit dem Tod auseinander?

\section{...Do you think about death?}

14 GB: Habe ich sehr intensiv. Also durch die Krankheit. Ich hatte ja mit 59 Jahren einen Stent.

15 Ein Jahr später Blasenkrebs. So. Dann habe ich nach diesem Blasenkrebs, auch hier ad memoriam-. Mein Vater ist an Blasenkrebs gestorben, schlimm. Und als die bei mir den

17 Blasenkrebs festgestellt haben, da brach für mich eine Welt zusammen. Da sah ich mich 18 eigentlich schon so ungefähr über den Deister gehen. Und da bin ich ja eigentlich 19 ausgestiegen. Da war ich 60. Da bin ich eigentlich ausgestiegen und da hat mein damaliger

20 Chef, der CEO von der Metro, hat gesagt G., das machst du nicht. Ich gebe dir einen Vertrag 21 in der Schweiz, du bestimmst selber wieviel du arbeiten willst, $\mathrm{du}$ machst die 22 Aufsichtsratsmandate weiter, die du hast. Ich war im Aufsichtsrat der Metro Deutschland auch. Und ansonsten für Projekte, du sagst wie du dich fühlst, was du tun kannst und dann 
sage ich dir, wo ich dich brauche. So, dann habe ich noch fünf Jahre im Prinzip dann in, aus der Schweiz auch den Job gemacht. Aber nur noch halb, so ungefähr. Und genau nach fünf

3 Jahren, ich hatte jedes Vierteljahr, jedes Quartal meine Kontrolluntersuchung, Blasenspiegelung. Das ist ja auch nicht das angenehmste, ist ja nicht wie Zähne putzen. Aber regelmäßig alle paar Monate. Und bei der letzten, nach fünf Jahren war ein Rezidiv da. Wieder ein neuer Tumor. Der ging dann aber Gott sei Dank dann auch wieder einfach rauszunehmen. Das war Ende 2010. Und dann kam es heftig, sage ich mal, im Februar 2014 mit dem Lungenkrebs. Und das war, sage mal, eine Konstellation, wo die Ärzte ohne mein Wissen meiner Frau und meinen Kindern gesagt hatten, also das maximal drei, sechs Monate Lebenserwartung. Regeln sie alles. So. Das wusste ich nicht. Und ich war-, um auf die Frage ganz spezifisch zu kommen. Ich war im Krankenhaus mit dem Lungenkrebs. Und die wusste dann nicht, was sie jetzt konkret machen sollten. Und dann habe ich im Bett gelegen. Und wenn du im Krankenhaus bist, sag ich mal, dann döst du den Tag über und nachts kannst du nicht mehr schlafen. Dann habe ich da wach-. Ich habe meine Beerdigung geplant, ich habe geplant wer was kriegen soll. Bis hin zur Musik, die ich haben wollte in der Kapelle, in der Grabeskirche. Und dann habe ich irgendwann gesagt, und dann immer-. Weißt du. Dann kommen die Anfälle dann, wo du eigentlich nur noch in Tränen ausbrichst. Ich sehe nicht mehr wie meine Enkel groß werden und, und ... Und da habe ich auch nur gesagt, Herrgott, ich kann das nicht bewältigen. Das ist für mich zu viel. Ich lege es in deine Hand. Du entscheidest und was immer du entscheidest nehme ich an. Lisa, von dem Augenblick an, hatte ich kein Problem mehr. Ich hatte eine innere Ruhe, das war wirklich Wahnsinn. Und ich war aber in einem Zustand, wo ich einfach gesagt habe, Herrgott, ich danke dir. Ich weiß, dass ich ..., daran sterbe ich nicht. Du willst mich noch nicht-. Ja, aber ich meine der Professor hat dann irgendwann gesagt, Herr B. 60 Prozent der Heilung ist Psyche. Aber der Heilungsprozess bei mir war für die Ärzte völlig unersichtlich.

And then I lay in bed. And when you are in hospital, let's say, you doze all day and then can't sleep at night. Then I lay awake... I planned my funeral; I planned who would get what. I chose the music I wanted to have in the chapel, the Church of the Holy Sepulchre ... And then there are the attacks when you only cry. I will not see my grandchildren grow up ...And then I only said, God, I cannot handle this. That is too much for me. I'll leave it to you. You decide, and whatever you decide I'll accept. Lisa, from this moment on, I had no problem anymore. I had such inner peace; it was incredible. And I was, however, in a state in which I simply said: God, thank you. I know that I..., I will not die. You don't yet want me. Yes, well, the professor at some point said, Mr B. $60 \%$ of the recovery is psychological. But my recovery was totally unexplainable to the doctors. 
LB: Hast Du dennoch Angst vor dem Tod?

Do you nonetheless fear death?

3 GB: Also man muss jetzt ehrlich sagen jain. Also schon ja, weil-. Also sagen wir so ganz

4 sicher bist du ja nicht. Und insbesondere, wenn man so eine Krankheitsgeschichte hat, wie

5 ich dann denkst Du natürlich auch oft daran wie wird das sein. Aber dann versuchst du auch

6 wieder das so ein bisschen von dir zu drücken. So nach dem Motto. Man muss jeden Tag,

7 also die einzige Realität die es gibt, ist jetzt und hier. So. Und wie du dann mit dem Tod 8 umgehst, sage ich mal, oder überhaupt. Ob du es mitkriegst ist dann, wenn es soweit ist.

9 Angst davor zu haben. Sicher ist, dass man irgendwann nicht mehr da ist. Und wenn ich 10 überlege, ich habe ja schon einiges an Tod erlebt, auch in meinem Leben, und an Tod 11 gesehen, dann ist das immer Schock und Erschrecken. Und ein extremer Verlust. Wo du dir 12 sagst, wie kann es sein, dass du das nicht mehr rückgängig machen kannst. Das ist diese 13 Endgültigkeit, die einen auch so dann tief trifft und erschüttert. Und wenn man selber dann 14 ist, und wenn man sich dann vorstellt. Mensch, das war meine Horrorvorstellung. Ich kann 15 die Anna nicht mehr zum Altar führen, wenn sie heiratet. Weil das einer der großen Wünsche 16 ist, die ich habe. Aber es ist keine Angst. Es ist auch ein Stück weit Vertrauen in das, was 17 dann kommt. Nicht Sicherheit. Soweit kann ich nicht gehen. Es ist ein Quäntchen Angst, weil 18 Unsicherheit. Aber auch ein Quäntchen Hoffnung oder Vertrauen. Diese Gemengelage hast $19 d u$.

Well, I have to honestly say yes and no. If only because... Well, let's say you are not one hundred per cent sure. And especially with a medical history like mine then you often think about how that will be. But then you also try to push that away from you a little bit. According to the principle: ...so the only reality that is there is here and now... The only thing that is certain is that one day you won't be there anymore...It is the finality that hits and shocks you so deeply. And when you yourself are, when you imagine this. Men, that was my horror scenario. I can't walk A. down the aisle when she gets married because that was my biggest wish. But it is no fear. It is partly trust in what there is to come...It is a grain of fear because of insecurity. But also a grain of hope or faith. That is the situation.

20 LB: Ich meine Du hast da ja schon einige Erfahrung. Aber die Vorstellung, dass Du-. Also 21 wenn dich nochmal eine Krankheit treffen würde, wäre es für dich schlimmer, wenn du körperlich eingeschränkt wärst oder geistig? 
...If you were hit by another illness, would it be worse for you to be limited physically or mentally?

1 GB: Also schlimmer wäre es für mich, wenn ich körperlich eingeschränkt wäre. Ganz klar,

2 weil ich erlebe das jetzt wirklich, also nicht direkt, aber durch meinen Bruder, mein ältester

3 Bruder, dessen bester Freund, der ist jetzt auch Ende 70, war Sportlehrer, Marathonläufer,

4 Tod und Teufel. Also sportlich sein ganzes Leben lang. Der ist jetzt seit Monaten, also diese

5 Krankheit, wo du körperlich-. Also der ist wie gelähmt. Das ist diese zunehmende

6 Muskelschwund oder was. Also zum Schluss wird der ersticken, weil die Lunge nicht mehr

7 funktioniert-. Also der ist seit Monaten-, kann der sich nicht bewegen und muss gefüttert

8 werden. Der muss auf die Toilette gebracht werden, der muss gewaschen werden, der muss

9 angezogen werden, der muss zum Tisch im Rollstuhl gebracht werden, damit der gefüttert-.

10 Wenn der Zeitung lesen will, dann muss einer dabei sein, der ihm umblättert. Und der Mann 11 ist geistig aber voll da. Und das stelle ich mir fürchterlich vor. Da sage ich ganz ehrlich, da

12 würde ich als vorbeugende Maßnahme würde ich mir die Tablette besorgen. Die kostet 5000

13 Euro.

Well, it would be worse for me if I was physically limited. Clearly, because I have experienced that factually, not directly, but through my brother, my oldest brother, his best friend, he is now end 70, was a sports teacher, marathon runner. So sportive all his life... He for months now has to, ... he cannot move and needs to be fed. He has to be taken to the toilet; he has to be cleaned, he has to be dressed, he has to be taken to the table in a wheelchair so that he can be fed. If he wants to read the newspaper, someone needs to turn the pages for him. And the man is mentally fully there. And that I imagine being terrible. I can honestly say, I would get, as a precautionary measure, get the tablet. It costs 5000 Euro...

14 LB: Ist das so?

Really?

15 GB: Ja, die kannst du in der Schweiz kriegen und in Holland auch. Wo du dann wirklich 16 sanft einschläfst. Also nicht mehr wach wirst. Abgesehen von diesem Schrecken, den du 17 selber dann als der Betroffene empfindest, wäre das für mich auch für mein Umfeld eine 18 unsägliche Belastung. Also die Frau von ihm, sage mal, die geht mittlerweile schon am 19 Stock. Die haben also jetzt rund um die Uhr eine 24 Stundenbetreuung aus Polen. Und 20 abgesehen davon, dass es enorm Geld kostet, müssen die alle zwei Monate gewechselt 21 werden, weil das körperlich für die auch gar nicht mehr machbar ist dann. Aber deshalb sage 22 ich ja, wenn du dann auch noch geistig. Wenn ich geistig nicht mehr da bin, was dann mit 
meinem Körper passiert, ist mir relativ egal. Das kriege ich nicht mehr mit. Aber wenn ich also geistig noch entscheidungsfähig bin und weiß, dass mein Körper nicht mehr mitmacht. Und dann ist für mich die Lebensqualität dermaßen heruntergefahren, dass ich da nicht mehr leben wollte.

If I am mentally gone, I don't care much about what happens to my body. I won't notice that. But if I am still capable of making decisions and know that my body can't carry its weight. And then the quality of life would have decreased so much for me that I would not want to continue living.

5 LB: Ja, das ist-. Wobei man ja auch bei diesen ganzen Demenzgeschichten gar nicht weiß-. 6 Auch da weiß man ja nicht, was kriegen die Leute tatsächlich noch mit? Also man würde 7 wünschen, dass es so ist, dass sie es nicht mitkriegen. So. Anderes Thema. Wie denkst Du $8 \quad$ ist die Einstellung in Deutschland zum Alter? Also gesellschaftlich.

...What do you think is the attitude towards older age in Germany? On a societal level.

9 GB: Also ich sage mal, diese Idee ist, ich sage mal geteilt. Ich denke, dass die ältere 10 Generation zunehmend Angst vor der Zukunft kriegt für sie selber. Das Thema Altersarmut, 11 sage ich jetzt mal, wird immer größer. Das wird noch verstärkt werden, durch die 12 zunehmende Zahl der Rentner aus der ganzen demografischen Entwicklung. Wenn die 13 ganzen geburtenstarken Nachkriegsjahrgänge jetzt zunehmend in Rente gehen, wird das, ich 14 sage mal, die Belastung noch umso größer. Und wenn du siehst, wie sich, ich sage mal, die 15 Lebenserhaltungskosten entwickelt haben, jetzt bei Miete und allem Drum und Dran. Musst 16 du ja sagen, dass ein Mann der sein ganzes Leben lang gearbeitet hat und eingezahlt ist, der 17 heute dann zwölf, fünfzehnhundert Euro Rente kriegt, der kann kaum davon leben. Wenn du 18 für eine kleine Zweizimmerwohnung mittlerweile acht-, neunhundert Euro Miete zahlen 19 musst, wie willst du denn davon leben? Also das ist das eine. Das andere ist, das ich denken 20 die jungen Leute die junge Generation wird aus dem gleichen Grunde natürlich zunehmend damit auch ein Problem haben, mit wachsender Belastung aus Sozialbeiträgen dafür, sagen wir mal, zur Kasse geben zu werden. Ich glaube, dass der Konflikt der Generationen aus der wirtschaftlichen Situation sich verstärken wird. Ich glaube, dass die zunehmende Schere arm und reich zu so starken Verwerfungen auch führen wird... Ich habe immer gesagt-. Nicht immer. Ich sage, was die Aristokratie im späten Mittelalter war. Wo irgendwann, sage ich jetzt mal, die Bauernkriege dann angefangen haben, weil die arme Bevölkerung nicht mehr mit ansehen konnte, dass die paar Aristokraten sich alles unter ihre eignen Krallen geschaufelt hat. Dass die Aristokratie von damals im Prinzip die Finanzinstitute und die, ich 
sage mal, Industrie, internationalen, globalen Player von heute. Da kommt wieder mein

Beispiel Quandt. Auch das wird zunehmend zu einer Polarisierung, reich, arm, alt, jung. Das wird zunehmen. Und dann kommt noch das ganze Migrationsthema dazu. Denn, wenn du heute in den Nachrichten gehört hast, welche Zustände mittlerweile an den Schulen herrschen. Dass wir eigentlich gar nicht mehr den Lehrstoff richtig vermittelt kriegen. Wir haben 20, 30, 40 Prozent in der Klasse sind Migrationshintergrundkinder, die kein richtiges Deutsch lernen. Und nicht von Zuhause aus, sage mal, das gilt nicht nur für die, auch für deutsche Kinder, keine Erziehung mehr genießen. Es kommt zu Gewaltexzessen an den

9 Schulen. Drogenmissbrauch, weiß der Teufel was. Keinen Respekt mehr vor den Lehrern 10 und Mitschülern erst recht nicht. Dann ist das eine Verrohung der Umgangsformen, die dann 11 extrem gefährlich ist. Dann werden die Alten, die Jungen zunehmend nicht mehr verstehen. 12 Und die Jungen werden den Respekt vor dem Alter zunehmend verlieren. Auch um selber, 13 sage ich jetzt mal, noch einigermaßen über die Runden zu kommen. Das ist für mich das 14 größte Risiko. Die soziale Ungerechtigkeit ist das größte Risiko für dieses Land.

I think that the older generation will feel increasingly worried about their future. The topic of poverty among the elderly, let's say, will increase. ... That will be intensified by the increasing number of pensioners from the whole demographic development. ... One has to say that a man who worked his whole life and paid social security and who then today receives a pension of twelve or fifteen-hundred Euro, he can hardly live of that. ....The other thing is that I think that the young people, the younger generation will for the same reasons have a problem, with the growing strain of social insurance contribution, to be soaked for that. I believe that the conflict between generations will increase as a result of the economic situation....Then the old will increasingly not understand the young ....and the young will more and more lose respect for older age. Also so they can somehow make ends meet. ... I believe that the increasing gap between poor and rich will lead to massive social upheaval...That is for me the greatest risk. Social injustice is the biggest risk for this country.

15 LB: Ja. Sehe ich ähnlich. Aber in deinem unmittelbaren Umfeld empfindest Du das nicht?

Yes, I view that similarly. But in your direct environment, you don't experience that?

16 GB: Nein. Absolut nicht. Wo ich manchmal mit kokettiere ist, wenn ich meiner Tochter sage und meinem Sohn sage: Also passt auf, ihr seid zwar jetzt im vollen Saft und seid auch schon arrivierte Manager und verdient gutes Geld aber ich bin noch nicht senil. Also einigermaßen

19 denken kann ich auch noch. Weil das hat schon Mal so ein bisschen manchmal die Attitüde, 20 dass du den Eindruck hast, deine Kinder glauben jetzt, sie müssten für dich jetzt 
1 Entscheidungen treffen, weil du selber nicht mehr in der Lage bist, das richtige zu tun. Aber

2 ansonsten sage ich mal nein.

No, absolutely not. What I sometimes jokingly say to my daughter and to my son is: Listen, you are now in your prime and also experienced managers, and you earn good money, but I am not senile. I can still think straight. Because sometimes there is somehow the attitude that you have the impression that your children now believe that they need to make decisions for you because you are not able to do the right thing yourself. But besides that, let's say, no.

3 LB: Aber Du hast ja auch eine Menge Erfahrung mit anderen Kulturen. Hast Du den

4 Eindruck, dass das in asiatischen Ländern zum Beispiel anders ist? Dass Alter da anders 5 gesehen wird?

...Do you have the impression that, for example in Asian countries, this is different? That they view older age differently?

6 GB: Ja. Aber ich sage mal so, wenn du Japan nimmst. Japan ist ja fast kaputt gegangen an 7 genau dem Konflikt, dass die alten, traditionellen Familienbande durch die Entwicklung der 8 Gesellschaft und der Wirtschaft komplett aufgelöst wurden. Die jungen Leute, sage ich jetzt 9 mal, die ausgeschert sind. Die alten plötzlich alleine waren und dieser Bruch in der sozialen, 10 gewachsenen Form hat das Land ja in Riesenprobleme geführt. Und wenn du China nimmst 11 als Beispiel. Was in China ja nach wie vor völlig, sagen wir mal, unangetastet ist, ist der 12 Respekt der Jugend vor dem Alter. So. Und selbst wenn das jetzt ein Multimilliardär, the 13 New-Rich in China ist. Wenn es dann zu den Eltern geht, dann macht er den großen Kotau 14 vor seinem Vater, vor seiner Mutter. Und das ist einfach eine Selbstverständlichkeit. Sagen 15 wir mal so, die asiatischen Kulturen sind noch sehr viel mehr, die sind traditionell 16 Generationengeflecht verbunden und verwachsen, als wir. Bei uns ist das schon sehr stark 17 entkoppelt. Und das siehst du ja. Jetzt nimm, meine Lebensspanne von 70 Jahren. Ich bin 18 groß geworden in einem drei Generationen Haushalt. Das gibt es heute überhaupt nicht 19 mehr. So. Und das ist schade, weil ich glaube, dass auch heute die Generationen 20 voneinander profitieren können. Also ich habe das als Kind erlebt, sage ich mal. Meine 21 Eltern haben gearbeitet, die Oma hat für uns gekocht, wenn wir aus dem Kindergarten oder 22 aus der Schule kamen, war immer leckeres, warmes Essen auf dem Tisch. So. Und das war 23 für uns super. Und es war für unsere Großeltern, sage ich mal, die war immer mitten dabei, 24 in der Familie. Das hat die auch so ein bisschen aktiv gehalten, jung gehalten. Wenn ich 25 heute die Eltern von der Tamara sehe. Deshalb haben wir uns das Haus heute angeguckt, 26 weil ihre Eltern damit reingehen würden. Die sitzen beide, sie sind beide 81, da passiert hier 
nichts mehr. Sie sitzt vor dem Fernseher und guckt ihre Soaps. Er sitzt vor dem Fernseher und guckt seinen Sport. So. Und ich meine die sind 65 Jahre verheiratet, da ist auch nicht mehr viel Neues, was es da auszutauschen gibt. Und wenn ich mir aber jetzt vorstelle, dass die mit uns zusammen sind, dann isst man zusammen, dann gibt es automatisch Gesprächsbedarf und Gesprächsaustausch. Das hält die jung und ein bisschen wirklich am Leben noch. Und für uns ist es ja auch nicht schädlich wenn man sich mit seinen Eltern dann beschäftigt oder unterhält vielleicht spielt man sogar mal am Abend eine Partei Romme oder was. Und sitzt nicht immer nur stur vorm Fernsehen oder vor dem I-Pad. Das ist ein Stück weit heute einfach verloren gegangen. Ich glaube nicht, dass das zurückzuholen ist. Ich glaube, das wird sich so weiterentwickeln. Es ist nicht mehr meine Welt. Ich könnte mir auch nicht vorstellen, dass ich da glücklich bin. Aber die Kinder wachsen da rein. Du siehst ja heute, wenn du in ein Restaurant gehst. Guck dir die Tische an. Da sitzt ja fast jeder, selbst, wenn drei, vier Leute am Tisch sind. Jeder hat sein Handy und guckt da ins Handy rein und schickt irgendwelche SMS oder sonst was. Aber das die sich unterhalten ist ja eher die Ausnahme als die Regel.

...Japan was nearly destroyed by exactly that conflict, that the old, traditional family bonds dissolved completely as a result of the developments in the society and economy. The young people that, let's say, stepped out of line. The old ones that were suddenly alone and this break in the socially established way has led the country into major problems. And if you take China as an example. What is still, let's say, totally untouched in China, is the respect the younger ones have for older age. ...And that is simply a matter of course. Let me say it like this, the Asian cultures are still much more, they are much more linked and grown together in generational networks than we are. With us this really quite degenerated.... They both sit, they are both 81 , nothing happens there. They both sit in front of TV and watch their soaps. He sits in front of TV and watches sports. So. And I mean they have been married for 65 years, there are not many new things to exchange......It is not my world anymore. I also can't imagine that that would make me happy. But the kids grow up with this. You see that today when you go into a restaurant. Look at the tables. Nearly everybody even when there are three, four people on the table. Everybody has his mobile phone and looks at his mobile phone and send some SMSs or something like that. But that they talk to each other is more the exception than the rule. 
No? Because?

1 GB: Ich glaube, dass das kann man so nicht verallgemeinern. Ich fühle mich nicht so als der

2 typische Rentner.

I don't think you can generalise this. I don't feel like the typical pensioner.

$3 \quad$ LB: Was ist denn der typische Rentner für dich?

What is a typical pensioner for you?

4 GB: Der typische Rentner für mich ist eigentlich der, für den alles sortiert ist. Der weiß, dass

5 er mit dem Geld, was er hat auskommen kann. Und der hat sein bezahltes Häuschen oder

6 eine Miete, die überschaubar ist. Und der kann, was weiß ich, da ein paar Tage in Urlaub

7 fahren oder was. Und der hat sein Fernsehen, seinen Sport, seine Nachrichten oder was. Also

8 geregelten, überschaubaren Ablauf. Und auch keine großen Ambitionen und Erwartungen

9 mehr. Das ist für mich eigentlich so der typische Rentner und so sehe ich mich nicht. Und

10 ich sehe oft, sage mal, dass der typische Rentner relativ große Distanz schon zu der jungen

11 Generation hat. Da ist ein Bruch. Und den sehe ich auch nicht für mich. Und deshalb sehe

12 ich mich nicht als typischen Rentner. Ich fühle mich da auch nicht repräsentiert.

The typical pensioner for me is somewhat the one for whom everything is sorted. Who knows that he can manage with the money he has. He paid off his little house, or a pays a manageable rent. And he can, whatever, go on holiday for a few days. And he has his TV, his sports, his news or something like that. So a regular, manageable routine. And also no high ambitions and expectations. That's what defines the typical pensioner for me, and that is not how I see myself. And I also often see that the typical pensioner has a big distance to the younger generation. There is a gap. And that one I also don't see applicable to myself.

13 LB: Aber von solchen Filmen, wie The Expendables, oder so? Kannst Du dich da rein-.

But such films as The Expendables, or something like that? Can you ...?

14 GB: Doch. Ja. Weil ich meine da ist ja doch noch-. Das hat ja Sinn. Da ist ja irgendwie noch 15 ein Inhalt, wo du sagen kannst, boah. Also trotz Alter. Die bringen es noch. Die haben kein 16 Selbstfindungsproblem. Oder die haben kein Selbstmotivationsproblem. Die wissen, dass 17 sie alt sind und die wissen, dass das, was sie, sage mal, können auch eine natürliche Grenze 18 hat. Aber im Rahmen dieser Grenze machen die voll mit. 
Sure. Yes because, I mean, there is somehow still ... That has meaning. That is somehow with substance where you can say boah. Well, despite older age. They are still getting somewhere. They have no problems with their self-identification. Or they have no problem with motivating themselves. They know that they are old and they know that, let's say, their abilities have a natural limit. But within these limits of their abilities, they fully take part.

1 LB: Ja. Glaubst Du, dass es grundsätzlich schwerer ist für Männer, oder für Frauen alt zu werden?

Yes, Do you think it is more difficult for men or for women to get older?

3 GB: Eigentlich glaube ich für Männer ist es schwerer.

Really, I believe it more difficult for men.

4 LB: Nein, ist nicht dein Ernst. Bist der Erste, der das sagt und das erfreut mich sehr.

You are kidding. You are the first one saying that and that pleases me a lot.

5 GB: Nein, also ich bin überzeugt, dass es für Männer schwerer ist alt zu werden. Also 6 Männer, sage mal, nach den alten Erziehungsklischees, dann auch am ehesten mit ihrer nachlassenden Kraft und nachlassenden Exposure, ein Problem haben. Wenn du irgendwo gewohnt warst als der Millebär, oder der große Draufgänger da durch Leben zu gehen und

9 jetzt bist du plötzlich ein altes Männchen dann hast du deine Probleme. Ich glaube auch,

10 dass Frauen sehr viel mehr innere Balance haben und insofern auch mit dem Alter besser

11 klarkommen. Sobald sie mal die Schwelle überwunden haben, dass äußere Schönheit und

12 inneres altern irgendwo auch zusammengehören. Dass das eine das andere nicht kaputt 13 macht. Und, wenn sie über diese kleine, ich sage mal, menschliche Schwäche drüber sind,

14 dann sind Frauen aus meiner Sicht viel stärker und gefestigter, im Inneren. Also vor dem

15 Hintergrund war ich auch immer jemand, der gesagt hat Frauen sind eigentlich das stärkere

16 Geschlecht. Nein, Frauen haben es da leichter. Mal von ganz einfachen praktischen Dingen

17 abgesehen. Ich glaube, die können sich leichter versorgen als ein Mann. Die haben auch in

18 ihrem Leben, in der Regel sage ich mal, ja immer versorgen müssen. Andere und auch sich

19 selber. Deshalb ist das für die kein großer Unterschied. Wenn ein Mann im Alter anfangen 20 muss zu kochen und selber einzukaufen und sein Bett zu machen und zu waschen und zu

21 bügeln, hat der ein Problem. So. Deshalb denke ich insgesamt, also aus dem praktischen

22 Aspekt, also auch aus dem inneren Aspekt ist eine Frau eigentlich besser dafür ausgerichtet 23 mit dem Alter klarzukommen. 
I also believe that women have much more inner balance... Within this context, I have always been somebody who said that women are the stronger sex.

$1 \quad[\ldots]$ 


\section{J Interview \& Translated Extracts Dr Lars Bühring}

$1 \quad$ LB: Gut. So erste Frage: Was bedeutet Männlichkeit für dich?

2 Lars Bühring: Männlichkeit bedeutet Tatkraft, Zielgerichtetheit, tja, anderes Wort,

3 Aktivität, für andere verantwortlich zu sein. Letztlich ja eben auch mit den Konsequenzen

4 leben, die in seinem Umfeld in irgendeiner Weise von Bedeutung sind und sich damit zu

5 identifizieren und daraus eben auch dann die entsprechenden Konsequenzen und Aktionen

6 abzuleiten. Und eigentlich, das ist eigentlich so das Gegenteil von passiv.

Masculinity means drive, determination, well, another word, activity, to be responsible for others. Ultimately to be able to also live with the consequences, which in his surrounding in some way have significance and to identify himself with them and to derive, well, then the suitable consequences and activities. Well, really it is the opposite of passive

7 LB: Sehr schön ...Auch in Abgrenzung zu Frauen? Also besteht deine

8 Männlichkeitsdefinition auch durchaus in Abgrenzung zu Frauen?

...So does your definition of masculinity depend on the demarcation to women?

9 Lars Bühring: Zu einem großen Teil durchaus. Aber natürlich kenne ich auch Frauen auf

10 diese Abgrenzung nur bedingt zutrifft. Aber grundsätzlich würde ich das Männern was ich

11 gesagt habe eher zutrauen als Frauen.

Largely yes, absolutely. But, of course, I also know women to who this demarcation only applies to in a limited degree...

12 LB: Dieses Bild, diese Vorstellung von Männlichkeit, hat die sich bei dir auch entwickelt

13 durch Aufwachsen, durch Erziehung... hast du den Eindruck oder zu anderen Zeitpunkten?

This image, this concept of masculinity, has this developed through your bringing up, your education... do you think or at other points in time?

14 Lars Bühring: Eigentlich durch Aufwachsen und Erziehen eigentlich weniger sondern, weil

15 ich ihm im Laufe des Erwachsenwerdens notwendigerweise oder wie die Situation sich halt

16 so dargestellt hat immer selbständiger geworden bin und immer weniger bei Entscheidungen

17 Dritte also in dem Falle hauptsächlich meine Eltern oder Geschwister herangezogen habe

18 und auch heranziehen wollte. Deshalb hat sich dieses Bild eigentlich durch diese

19 Entwicklung langsam geformt und ist nicht von vornherein so gewesen. Wenn ich meine

20 Mutter angucke dann würde dieser Eindruck stark daher rühren weil meine Mutter ja in einer

21 gewissen Weise sehr passiv war aber während des Krieges eben unheimlich aktiv die

22 Familiengeschicke gelenkt hat. Und was meine Schwestern angeht, die haben dann durchaus

23 in unterschiedlichem Maße durchaus auch eigene Aktivitäten und eigene Akzente gesetzt

24 um sich dann letztendlich aber dann doch wieder $\mathrm{zu}$ sozusagen den allgemeinen 
1 Verhältnissen, das heißt also ihrem jeweiligen Mann unterzuordnen. Insofern denke ich hat

2 sich das im Laufe der Jahre langsam so geformt und also durch die durch die Jugend und so

3 eigentlich weniger.

That's why this conception ${ }^{29}$ really formed itself slowly through this development and was not like this from the start. When I look at my mother, then this impression would largely be attributed to the fact that my mother was in some way very passive, but during the war, she incredibly determinedly guided the family's destiny. And with regards to my sisters, they then were in different degrees able to have their own activities, set their own course to then in the end in some way subordinate themselves to the general standards, that means their respective husbands. Insofar I think that throughout the years it formed itself slowly and well, less during adolescence.

4 LB: Das heißt dein Vater hat auch diesem Männlichkeitsbild, was du eben erwähnt nicht 5 notwendigerweise entsprochen?

That means your father did not necessarily match this image of masculinity you just talked about?

6 Lars Bühring: Doch der hat ihm schon entsprochen. Aber der hat dem entsprochen. Aber

7 ich weiß nicht ob der unbedingt ein Vorbild für mich gewesen ist und ich deshalb diese

8 Geschichte angenommen habe. Denn dieses Vorbild oder sagen wir mal, das was mein Vater

9 sich... oder dargestellt hat das resultiert aus einer Zeit die teilweise noch aus kaiserlichen

10 Zeiten herrührt. Die hat einen ganz anderen Grund als Aktivität oder so was. Das ist mehr

11 autoritär gewesen. Das weiß ich nicht ob ich das, also ob ich das so für mich so wahrnehmen

12 wollte. Das war also nicht so gerade ein Vorbild was ich da ich... Ich hab das schon gesehen

13 dass mein Vater da viel zu kamellen hatte aber ich habe dann auch gesehen, dass er als meine

14 das dann als mein Vater älter geworden ist und meine Mutter sich mit zumindest einer

15 Schwester verbinden konnte, verbunden konnte, koalieren konnte, dass er da mächtig in die

16 Bredouille gekommen ist ja schon richtig ins Hintertreffen geraten ist also als kaiserlicher

17 Offizier dann soweit gekommen ist dass er mir erklärt hat wenn das so weiter ginge würde

18 er sich nach 50 Jahren scheiden lassen. Das heißt also dass diese absolute Dominanz und

19 Aktivität und sowas zu dieser Zeit von der ich jetzt spreche ich stark von meiner Mutter und

20 meiner Schwester untergraben worden ist.

He did match it, but he did match it. But I don't know if he really was a role model for me and I, therefore, adopted this story. Because this role model or let's say what my father represented resulted from a time that was partly the imperial period. There was a completely different reason than activity or something like that. That was more authoritarian. I don't know if I wanted to perceive it like that. That wasn't really a role model which I... I saw that

${ }^{29}$ Conception of masculinity 
my father was very much in charge $\mathrm{e}^{30}$ but I then also saw that when my father became older, and my mother could link up, cooperate with at least one of my sisters, that it got him into a major predicament, he majorly fell back, and as an imperial officer, it got so far that he told me that if this continued he would after 50 years of marriage get a divorce. That means that this absolute dominance and activity and such things were at the time I'm talking about majorly undermined by my mother and my sister.

$1 \quad[\ldots]$

2 LB: Kannst du bitte nochmal kurz deinen beruflichen Werdegang beschreiben.

Can you please briefly describe your professional career.

3 Lars Bühring: Beruflicher Werdegang war folgender: dass ich nachdem ich ein 4 Diplomexamen an der Universität Marburg gemacht habe im Jahre 1965 dann aufgrund, 5 nicht zuletzt aufgrund des Ergebnisses dieser, dieses Diploms auf ein Stipendium von der 6 VW Stiftung bekommen habe und, und mit diesem Stipendium angefangen habe zu 7 promovieren in Marburg. Diese Arbeiten an der Promotion, das wird jeder der da mal das 8 selber gemacht hat nachvollziehen können, sind also mit Sicherheit nicht unbedingt ganz 9 den Tag ausfüllend, das heißt nach einer gewissen Zeit, nämlich nach anderthalb Jahren 10 habe ich dann diese ausschließliche Beschäftigung mit der Promotion eingetauscht gegen 11 meine erste berufliche Tätigkeit bei einem Wirtschaftsforschungsinstitut in Bad Godesberg 12 und habe im Jahr 66 dort angefangen zu arbeiten und habe nebenbei, was natürlich dann 13 schon etwas, zeitlich etwas verzögert wurde bis ins Jahr 69 dann diese Promotion nebenbei 14 weitergemacht und im Jahr 69 dann promoviert. Im Jahre 1970 habe ich dann die Stelle bei 15 dem Forschungsinstitut aufgegeben und bin für drei Jahre, inzwischen mit Familie mit Frau 16 noch ohne Kind nach Hamburg übergewechselt und habe dort im Controlling Bereich bei 17 der Esso gearbeitet. Während dieser Zeit ist meine Tochter geboren worden. So, nach diesen 18 drei Jahren in denen ich was meine beruflichen was die beruflichen Anforderungen angeht 19 sehr, sehr, eigentlich so viel wie nie später gelernt habe, was man im Rahmen eines Ölmultis 20 nur hat lernen können, gerade auf diesem Gebiet Abrechnung, Steuern, Finanzen, Bilanzen, 21 habe ich weil diese Umgebung dieses Großkonzerns doch an vielen Stellen meinen 22 Vorstellungen sehr, sehr wenig entsprach, habe ich die Stelle aufgegeben und habe eine neue 23 Stelle in Köln bei der Firma Otto Wolff angefangen die ein mittelständisches Unternehmen 24 war mit vielfältigen Aktivitäten mit dem Hauptpunkt auf dem Gebiet Stahl und 25 Metallverarbeitung. Und habe dort in verschiedenen Funktionen vom Jahre 73 bis zum Jahr 2688 bin ich dann tätig geworden und habe im Rahmen dieser Tätigkeit ganz häufig 27 Auslandsreisen in sehr viele Länder Asiens, Afrikas aber, vor allen Dingen, Südamerikas 28 unternommen. So im Jahre 1988, oder sagen wir in diesen Jahren, ist mir dann klar geworden 29 dass eine Firma mit diesen mittelständischen Strukturen im Rahmen von Eisen und Stahl auf

${ }^{30}$ In the original transcript my father used the word 'kamellen' which is a colloquial word for being in charge 
1 Dauer keinen Platz haben wird und höchstwahrscheinlich in irgendeiner Weise entweder

2 untergeht oder aber von wesentlich Größeren übernommen wird. Mit der Folge dass man,

3 dass ich diese Umgebung, die ich für meine Tätigkeit eigentlich für relativ adäquat hielt dann

4 wohl verloren hätte. Es ergab sich die Möglichkeit dass ein früherer Chef von mir sich

5 selbstständig gemacht hat und mich und mir angeboten hat ob ich nicht bei ihm auch tätig

6 wäre. Das hab ich dann gemacht und bin zu ihm in ein, ja, in ein Freelance Verhältnis

7 eingetreten. Mit der Folge dass wir im Laufe der Jahre ein kleines und ein kleines

8 Unternehmensimperium uns zusammengekauft haben indem wir lauter mittelständische

9 Unternehmen gewerblicher Art übernommen haben und behutsam geführt haben und in ihrer

10 Struktur im Wesentlichen auch so belassen haben. Und das hat dazu beigetragen dass diese

11 Unternehmen durchaus weiter gewachsen und gediehen sind und wir selber damit natürlich.

12 Das war die letzte Station meines beruflichen Werdegangs. Ich bin von 1988 bis ich dann

13 endgültig.. bin bei dieser Firma gewesen, die Firma hat dann später ein..., ist dann später

14 Aktiengesellschaft geworden. Ich bin dann in dieser Aktiengesellschaft Finanzvorstand

15 geworden und habe dann auch noch hinterher beratenderweise insgesamt bis zum Jahr 2008

16 war ich mit dieser Firma verbunden

... since this environment of big corporations did in many ways not match my conceptions.

17 LB: Gleich noch mal zu Indus... aber weil du gesagt hast die Esso hätte dir nicht entsprochen,

18 was hat dir denn da nicht gefallen?

... since you said Esso wasn't your cup of tea, what exactly didn't you like there?

19 Lars Bühring: Dieses, dieses absolut völlig unpersönliche Verhältnis von... von Tätigkeit

20 und Menschen, die absolut völlig von der Hierarchie und von den Regelungen wurde das

21 Verhältnis der Menschen untereinander bestimmt und ließ also keine persönlichen

22 Ausprägungen oder Ausnahmen oder irgendwas zu. So dass man also durchaus sich selber

23 überhaupt gar nicht mehr wiederfand in dieser Tätigkeit, die er da gemacht hat. Und wenn

24 ich dafür... ich habe dann unter anderem Tätigkeiten verrichten habe an anderer Stelle mal

25 irgendwo weil ich mich da nicht recht auskannte, ich habe dann einfach, so wie mir das wie

26 mir das sinnvoll erschien, irgendeine, ein Ergebnis niedergelegt und weitergeleitet und bin

27 dann nach einem Vierteljahr auf diesen Fehler dann wieder zurückgeführt worden, was ich

28 denn da veranstaltet hätte. Das würde doch in keinem zusammen ...allerdings auch das

29 wieder, und das war typisch, eben nicht durch eine Person, sondern durch eine durch ein

30 anonymes Schriftstück, was an mich, was mir dann zugeleitet worden ist.

...This absolute totally impersonal relationship between activity and people, hierarchies and regulations absolute totally determined the relationship with each other and didn't allow for individual characteristics or something like that. So that he could not at all identify himself with this activity, which he did. 
2 LB: Fließender Übergang zur nächsten Frage: Was hat dir denn besonders Freude gemacht

3 bei der Indus, also deinem letzten Job?

...What did particularly enjoy at Indus, your last job?

4 Lars Bühring: Das, die, das was bei der insbesondere Freude gemacht hat, dass man, das 5 ich mir ganz wesentlich die Kriterien meines Handelns und die Grenzen meines Handelns

6 eigentlich mehr oder weniger selber definieren konnte und dass wir, der Kill und dann 7 hinterher der Rosenfeld, also der dritte Vorstand, uns untereinander in einem ganz weiten 8 Maße sehr gut verstanden haben und uns was das was die Basis von dem, was ich vorhin 9 gesagt habe, war gegenseitig total vertraut haben, was das man sich auf das was der andere 10 machen kann verlassen kann, das ist eine...das der verantwortlich mit seiner Freiheit umgeht.

That what I enjoyed especially there (at Indus) was that I could essentially define the crucial criteria for my actions and the limits of my actions more or less myself and that we, K. and then later Rosenfeld, so the third member of the managing board, got along very well and the basis of that was what I had said previously, that we trusted each other totally, that you could trust in what the other did, that it is a ... that the other uses his autonomy responsibly.

11 LB: Das heißt Hierarchien waren nicht notwendig weil ...?

That means hierarchies were not necessary because...?

12 Lars Bühring: Es gab praktisch keine Hierarchien und auch im Verhältnis zu den zu den

13 Gesellschaften und denen in diesen Gesellschaften verantwortlichen Geschäftsführungen

14 war die Hierarchie, oder sagen wir mal, die... dass das Winken mit der Hierarchiestufe

15 eigentlich im Verkehr kein Argument sondern das Argument war eigentlich immer

16 Überzeugung für, zu irgendwas oder aber etwas gemeinsam zu überlegen und gemeinsam

$17 \mathrm{zu}$ einer Lösung zu kommen und nicht von vornherein irgendwie eine vorgefasste, ein

18 vorgefasste Schema zu haben.

There were practically no hierarchies, also in the relationship to the companies and the management board responsible for them the hierarchy was... or let's say, that hinting at the hierarchical rank was not really an argument in the relationship, but the argument was always persuasion of something or else to think about something together and to find a solution together and to not have a preconceived schema from the start. 
1 LB: Und findest du ... war dir das angenehme oder eher unangenehm also dass die Leute

2 schon einen gewissen Respekt entgegengebracht haben, nicht dir persönlich als Mensch

3 sondern deiner Position.

And do you think...did you find that pleasant or unpleasant well that people paid you certain respect, not you as a private person but your position?

4 Lars Bühring: Ich verstehe das schon aber eigentlich waren mir das weder angenehm noch 5 unangenehm, sondern vielmehr mir war das klar. Das war einfach so. Ich kann mich nicht 6 dagegen wehren aber ich finde das auch nicht besonders toll.

I understand that somehow but really I found it neither pleasant nor unpleasant but it was simply aware of it. That was the way it was. I can't defend myself against it, but I also don't find it particularly great.

7 LB: Wie war, hat sich denn so ein Privatleben gestaltet werden während du berufstätig warst 8 also bedeutet Zeit, wieviel Zeit, was hast du gemacht in deiner freien Zeit, wieviel Zeit hast 9 du im Beruf verbracht?

How was your private life structured when you were employed, so that means how much time... what did you do in your free time and how much time did you spend on the job?

10 Lars Bühring: Das war ein für meine Begriffe ein, ein weiteres ganz starkes Argument für 11 die letzten 20 Jahre, dass wir, in einer unausgesprochener Weise dazugekommen sind, dass 12 wir in der Woche, was ich meine, von Montag bis Freitag, praktisch für unsere Tätigkeit 13 mehr oder weniger keine zeitliche Begrenzung gehabt haben, also nicht eine Steckkarte oder 14 Lochkarte im Hinterkopf hatten wir. Das wir aber davon ausgegangen sind, alle, alle drei 15 und das auch einfach durchgeführt haben, dass das Wochenende ein privater, eine private 16 Sache ist dass wir im Wesentlichen zwei Tage in der Woche frei haben, mal von gewissen, 17 wenn wir mal groß irgendwelche Termine hatten die am Montagmorgen begannen dann sind 18 wir schon am Sonntagnachmittag oder Sonntagabend weggefahren aber das war die große

19 Ausnahme. Und das Weitere war, dass wir alle in einem durchaus ausreichenden und sehr, 20 schon sehr großzügige Rahmen alle unsere Urlaube gemacht haben. Wir haben nicht sagen 21 können wie das bei anderen Stellen, sagen wir in ähnlich, vergleichbarer Größenordnung, 22 die dann sagen ich habe praktisch noch ein halbes Jahr Urlaub bekommen weil ich so viel 23 noch, ich kann nicht, ich kann nicht weg. Das war bei uns nicht der Fall.

...without needing to explicitly talk about it we all agreed that during the week, what I mean, from Monday to Friday, there was practically no time limit for our activities, so we did not have a time card or punch card in the back of our minds. But we agreed, all, all three and also just executed this, that the weekend is a private, a private thing, that essentially two days of week are off....Another thing was that we all took holidays in an adequate, more than adequate time frame... 
$2 \quad$ LB: Und das macht dir Spaß?

And that is fun?

3 Lars Bühring: Das ist so ganz schön.

That is the way, it is quite nice.

$4 \quad$ LB: Was macht dir denn daran Spaß?

What do you enjoy about it?

5 Lars Bühring: Das macht daran Spaß das es, das ich sehe dass ich bestimmte Eigenarten 6 oder Fähigkeiten die ich halt habe, dass ich die im Rahmen dieser Stiftung relativ sinnvoll 7 einsetzen kann und, dass diese Stiftung, eine der größten privaten Stiftungen in Deutschland, 8 dass die, so wie ich das sonst so festgestellt habe, sehr oft mehr oder weniger auf auf dem

9 Niveau von so Kleingartenverein durchgeführt wird und das ist natürlich sehr schade für, in 10 dem Falle, wirklich große, große Vermögen, die in diese Stiftung eingebracht werden, dass 11 das nur in Ansätzen professionell gehandhabt wird und teilweise auch an den Interessen 12 Gegensätzen, der Leute, die sich da in dieser, in dieser Stiftung wiederzufinden glauben dann 13 damit verkommt. Das also der Sinn dessen was der Stifter, die Stifter damit mal bezweckt 14 haben wollen immer weniger zum Ausdruck kommt und dass also die Grabenkämpfe und 15 damit letztlich dann also eine Vergeudung von Aktivitäten und letztlich auch vom Geld dann 16 dazu kommt, dass also die Stiftung nicht das erreicht was sie erreichen könnte.

What I enjoy about it is that I see that certain characteristics or abilities that I happen to have, that I can apply them within the frame of this foundation quite effectively and that this foundation, one of the biggest private foundations in Germany, that they, as I found out, very often are led more or less on the level of an allotment association, and that is obviously a shame for, in this case, really big, big assets, that are placed in these foundations, that, that are only partially handled professionally... That therefore what the founder, the founders wanted to achieve is realised less and less and that the resulting trench warfare and with it the waste of energy and also money leads to the foundation not achieving what it could have achieved.

17 LB: Wie hast du denn den Übergang vom Berufsleben zum Rentnerdasein erlebt? Wie war 18 das für dich?

How did you experience the transition from employment to retirement? How was that for you? 
1 Lars Bühring: Ganz toll weil, dass muss ich jetzt noch sagen, so schön also diese ganze

2 Zeit war und die Woche vorher beruflich eben durchaus angespannt so dass der Abend,

3 zumindest in der Woche, zu großen anderen Aktivitäten oder großen anderen, ja ich sag mal,

4 großen geistigen Anstrengungen eigentlich weniger in Frage kam. So dass eins in dieser

5 langen Zeit, für meine Begriffe, ganz wesentlich zu kurz gekommen ist und das war Lesen.

6 Und das habe ich also so unglaublich genossen dass ich die Möglichkeit habe mir meine

7 Aktivitäten vom Aufstehen bis zum ins Bett gehen durchaus selber aussuchen kann und

8 aussuchen konnte und die auch strukturiert haben konnte. Und das habe ich in der ersten Zeit

9 habe ich auch, in der ersten Zeit, ich sag mal, die ersten drei, vier Jahren oder sowas, habe

10 also ich auch unglaublich viel gelesen und zwar ganz rundum von Belletristik über

11 Sachbücher Bücher hauptsächlich mit Schwerpunkt Geschichte und fand das... und darüber

12 hinaus haben wir was wir dann natürlich auch nicht so gemacht haben weil die die

13 Urlaubszeiten eben weitgehend mit, ja, mit Inhalten sportlicher, sportlicher Provenienz,

14 zumindest im Winter ausgefüllt waren, wir haben dann auch durchaus sehr viel und sehr

15 unterschiedliche Reisen unternommen. Nach Ägypten und nach Russland und nach Italien,

16 Spanien und Frankreich sowieso und eben auch nach Kanada. So.

Great because, I have to say that, as great as the entire time was, previously the weeks had professionally been rather tense so that the evenings, at least during the week, did not offer themselves for other major activities or major, let's say, mental efforts were out of the question. So that one thing, to my mind, was neglected massively and that was reading. And I enjoyed it immensely to have the possibility to choose and structure my activities from morning till evening autonomously. And that I in the beginning, in the beginning, I, let's say in the first three to four years or so, I read a lot and everything from fiction to non-fiction but predominantly books with a focus on historical developments, and I found this ... in addition what we did ... what we, of course, did not do so much since holiday times were filled with, well, content of a sportive character, at least in winter, we additionally undertook different journeys. To Egypt and to Russia and to Italy, Spain and France anyways and also to Canada.

17 LB: Hattest du oder hast du den Eindruck dass deine Position als Rentner im Vergleich zu

18 deiner Position früher bei der Indus einen Einfluss darauf hat wie die Leute dich 19 wahrnehmen, Außenstehende?

Did you or do you have the impression that your position as a pensioner in comparison to your previous position at Indus had/has an influence on how people, outsiders, view you?

20 Lars Bühring: Ich hab so den Eindruck, also gerade eben Außenstehende, so ganz kann 21 man das eigentlich einfach nicht ablegen. Das heißt, dass schleift man noch mit sich rum. So 22 praktisch als Neugeborener Rentner taucht man eigentlich nie auf. 
I have the impression, especially with outsiders, you can't get rid of this totally. That means you take that with you. You don't ever appear as a new-born pensioner.

1 LB: Das sind aber dann Leute die dich sozusagen aus der Indus Zeit auch kennen, also die 2 sozusagen ja damit auch sozusagen in Verbindung bringen?

But these are people who used to know you in your time at Indus so that they somehow link you with it?

3 Lars Bühring: Nein, nein, nicht, nicht unbedingt. Nee, also ganz, ganz albernes Beispiel, 4 ich habe nun mit meinen früheren Klassenkameraden relativ wenig am Hut weil ich da von

5 so Klassentreffen nicht so viel halte aber ab und zu kommt dann halt mal eben so einer der

6 mich aus früheren, mit dem ich zusammen Abitur gemacht habe, und da kommt das also

7 durchaus zum Ausdruck dass ich das, was ich mal beruflich gemacht habe, bei dem also zum

8 Beispiel durchaus eine Rolle ist oder ein Punkt ist der mit zur Beurteilung beiträgt.

No, no, not necessarily. No, well a silly example, I have relatively little to do with my previous classmates ...but occasionally somebody turns up whom I did my A-levels with, then it really comes to light that what I used to do professionally really plays a role, well, is an aspect which contributes to his evaluation of mine.

9 LB: Ja, gutes Beispiel, hat es für dich selber einen Einfluss darauf gehabt, wie du dich siehst 10 als Mann, als Mensch?

...has it had an effect on how you view yourself as a person, as a man?

11 Lars Bühring: Was jetzt?

What exactly?

$12 \quad$ LB: Nicht mehr im Beruf zu stehen?

That you are not employed anymore?

13 Lars Bühring: Nö, ich kann eigentlich nur sagen dass ich das so akzeptiere, was ich für eine

14 Position im Augenblick habe oder was mir mein Leben im Augenblick ausmacht. Aber wenn

15 die Frage darauf hinausläuft ob ich mich jetzt sozusagen weniger wertig empfinde, oder so.

16 Dann muss ich sagen, dass ist nicht der Fall. Jede Zeit hat, hat ihre Funktion. Jede Zeit hat

17 auch ihre Besonderheiten und ihre Schönheiten und diese ist halt das, die finde ich also

18 überhaupt wunderschön. Und eines ist natürlich klar: zurück möchte ich überhaupt nie, never.

No, I can only say that I accept the position I have at the moment or what characterises life at the moment. But if the question aims at finding out if I feel less valuable or something like that. Then I have to say that is not the case. Each period has its function. Each period 
also has its particularities and its beauty and this one, well, I generally find wonderful. And one thing is for sure: I don't want to go back, never.

$1 \quad$ LB: Weil?

Because?

2 Lars Bühring: Weil ich natürlich dadurch dass ich mit einigen von meinen 3 Geschäftsfreunden von damals inzwischen befreundet bin und ich damit notwendigerweise 4 auch die Entwicklung in der Indus mitbekomme weil ich halt in dieser Art und Weise wie 5 das Geschäft im Augenblick da durchgeführt wird das ist mir nicht mehr gemäß. Das ist 6 etwas was völlig anderes möglicherweise nicht weniger erfolgreich aber es ist etwas was 7 mir nicht gemäß ist und was ich so nicht machen wollte.

...because the way that the business is conducted at the moment does not correspond with me. That is something totally different, possibly not less successful but it is something which does not correspond with me and what I wouldn't want to do.

$8 \quad$ LB: Sag nochmal warum genau?

Tell me again, why not?

9 Lars Bühring: Ja also, zu unserer Zeit haben wir, ich sag mal hinterher waren das 6000

10 Leute da und ich habe zu den Zeiten vielleicht tausend Leute mit Namen gekannt. Zu jetziger

11 Zeit, wenn ich mit einem von meinen Freunden da spreche, ist das so dass er von dem

12 zuständigen Vorstand, der ich damals bei verschiedenen Gesellschaften gewesen bin, dass

13 er von dem von dem zuständigen Vorstand allenfalls zweimal im Jahr und das am Ort, des

14 Sitzes von der Indus sieht und der überhaupt nicht mehr in die Firma kommt. Das heißt das

15 Interesse an den eigentlichen Tätigkeiten in der Gesellschaft, in den Gesellschaften wo, wo

16 auch wirklich das abläuft was von Belang ist, wo im Endeffekt auch das Geld verdient wird

17 und wo die Zukunft liegt, das ist wie auf der Strecke geblieben und ist weitgehend abgelöst

18 worden von einer immer weiter sich ausdehnenden Verwaltung und Kontrolle. Während zu

19 unserer Zeit waren wir bei 6000 Leuten, ungefähr 6000 Leuten, waren wir glaube ich mit

20 Fahrern und Sekretären unter zehn Personen. Heute sind das bei, ich weiß nicht, neuntausend

21 Beschäftigten oder so was, sind das weiß ich was 35 oder so. Das heißt es ist unter der Hand

22 ein Wasserkopf entstanden und die Tätigkeit eines Vorstandes bei der Indus die ist

23 inzwischen auf Dinge konzentriert, die mein großes Interesse nicht mehr findet.

That means the interest in the activities in the company, in the companies where something which counts is happening, where in the end the money is made and where the future lies, that was abandoned, and it was replaced by an ever-increasing bureaucracy and control... That means that on the quiet, a top-heavy bureaucracy has developed and the activities of the board of management at Indus concentrates on other things that do not find my interest. 
2 LB: Wie würdest du Alter also nicht Lebensalter also, sondern grundsätzlich Alter, 3 definieren. Was bedeutet das für dich?

How would you define older age, not chronological age, but older age in general? What does it mean to you?

4 Lars Bühring: Alter bedeutet für mich erst mal das man es nicht mehr eilig hat. Und nicht 5 mehr eilig haben heißt das dass man möglicherweise auch in seinen Reaktionen nicht mehr, 6 dass die Amplituden, in denen es, in seinen Aktionen und auch in seinen Reaktionen nicht 7 mehr so nach oben und unten ausschlagen kann. Das heißt also das absolute Entzücken und

8 die totale Verzweiflung die finden in dem in dem Ausmaß was Alter angeht nicht mehr so 9 statt.

Older age, first of all, means to me that you are not in a hurry anymore. And to not be in a hurry anymore means that you are possibly in your reactions not anymore, that the amplitudes in his actions and reactions cannot deflect up and down so much. That, therefore, means that with reference to older age total delight but also total despair do not happen as such anymore.

10 LB: Also schon in die Richtung Gelassenheit?

So in the direction of placidity?

11 Lars Bühring: Absolut, also ich will damit nicht, ich hab mir das versagt, jetzt sage ich 12 dann doch mal in Richtung Weisheit, aber so was. Das man sagt was hat das eigentlich, 13 irgendeine Geschichte nun wirklich, wenn man alles betrachtet, für einen Belang. Muss man 14 darüber einen Leberschadenbekommen. Nein.

Absolutely, well, I don't want to say, I refrained from saying, but now I'll say it anyway, in the direction of wisdom, but something like that...

15 LB: Wenn du, es immer sehr schwierig das zu fragen, aber als wie alt empfindest du dich 16 denn? Denn es ist ja nicht so, dieses chronologische Alter, das sagt ja nicht 17 notwendigerweise etwas darüber aus, wie man sich selber empfindet. ...How old do you feel to be?...

18 Lars Bühring: Also der erste Punkt ist dass ich mich das ich mich das natürlich sehr häufig 19 Frage und nicht einfach davon ausgehe dass ich einfach noch 35 bin. Das ist nicht der Fall. 20 Das ist überhaupt nicht der Fall. Das heißt bestimmte Sektoren bestimmte Anlässe, 21 bestimmte Dinge, die sind halt für mich nicht mehr adäquat, da also so. Also ich gehe nicht 22 mehr wenn es das auch nicht mehr gibt, aber ich gehe nicht mehr zu Talentprobe am 
Tanzbrunnen oder in eine Disco. Aber was das was das Fühlen angeht ist das für mich auch immer so ein bisschen der Spiegel wie die anderen mich sehen. Und wenn ich mal davon aus und wenn ich mal davon ausgehe dass zum Beispiel eben der Christian und der Peer, zwei 4 junge Männer, die jetzt so Mitte 40 sind und die... mit denen ich ganz viel in den langen 5 Jahren zusammen unternommen habe, meistens ob... hauptsächlich sportlicher Art, das die 6 also durchaus interessiert sind mit mir weiterhin Tennis zu spielen, einmal in der Woche.

7 Und dieser dieses Treffen einmal in der Woche für die durchaus eine gewisse Bedeutung hat, 8 dann muss ich sagen dann bin ich möglicherweise, auch in deren Augen und auch für mich, 9 noch nicht ganz so alt ist wie das Lebensalter zu sein scheint.(Telefonklingeln)

...But with reference to feeling it is for me always a little bit the reflection of how others see me. And when I consider, when I consider that to ...two young men, who are in their midforties and they ... that they are really interested in continuing to play tennis with me, once a week. And that these meetings once a week clearly have a certain significance to them, then I have to say, then I am possibly, also in their eyes and in mine, not quite as old as the chronological age seems to indicate.

11 LB: Kannst du es irgendwie festzurren an einem spezifischen Alter also es muss jetzt nicht aufs Jahr genau sein aber so...

Can you link it to a specific age, ...?

13 Lars Bühring: Also, ich meine wenn ich mal, ich, ich unterteile das mal zumindest in zwei 14 Kategorien. Das eine ist mehr oder weniger die Physis. Wenn ich mir meine Umgebung 15 angucke und die anderen die so ähnlich sind wie ich angucke, die ja mehr oder weniger 16 durchaus doch sehr, sehr an verschiedenen Stellen mit ihrem Körper zu tun haben und auch 17 sehr viele Ausfälle habe dann muss ich sagen, dann sind das ich sag mal so was bei zehn 18 Jahren, das ist also glaube ich kein..., sowas in der Größenordnung ist das. Und das andere 19 ist was ich auch so... In welchem Maße ich mich a für Dinge in der Welt interessiere oder 20 hauptsächlich oder in Fokussierung darauf ob und in welchem Maße ich bereit bin mich mit 21 irgendwelchen neuen Gedanken oder Entwicklungen oder sowas zu befassen. Das ist glaube 22 ich mehr so diese geistig, geistige Entwicklung. Und da glaube ich bin ich noch nicht auf dem Stand, dass ich sage das ist sowieso alles egal weil ich ja doch bald sterbe.

...When I look at my environment and when I look at the others who are similar to me who clearly have to do with different parts of their body and who also have many bodily deficits, then I believe it to be in the region of ten years, that is I think no ... it is something in that range... 
$1 \quad$ LB: Das heißt er ist grundsätzlich nicht das Gefühl dass sich deinen mentalen und seine

2 physische Kräfte verschlechtert haben.

So that means you don't have the feeling that your physical and mental abilities have declined?

3 Lars Bühring: Das dass die, die Physis und auch die, ja, sagen wir mal das Gedächtnis

4 durchaus sein Tribut zollen muss an den Jahren das ist überhaupt keine Frage. Ne, also

5 wenn ich früher mit dem Fahrrad gefahren bin da bin ich denke ich mal schon etwas schneller

6 gefahren als ich das heute kann ohne dass ich da gleich anfangen zu schnaufen oder sagen

7 wir mal jetzt fange ich früher an zu schnaufen als das früher der Fall war. Aber grundsätzlich

8 hält sich das noch in einem Rahmen das ich, ich sage mal, von Rollstuhl schon noch etwas

9 entfernt bin.

That the years take its toll on physical performance and also, let's say, the memory, that is no question...

10 LB Also du empfindest schon durchaus Veränderung, durch Alter?

So you have noticed changes due to older age?

11 Lars Bühring: Durchaus.

12 LB: Und wie ist das für dich? Wie gehst du damit um?

And how is that for you? How do you handle that?

13 Lars Bühring: Ja, ist manchmal lästig zum Beispiel wenn man ganz einfach was vergießt

14 oder nicht... Dann ist das einfach lästig. Aber es ist nicht so dass man, dass ich mich dann

15 vor Ärger in den Bauch beißen will. Das nicht der Fall. Aber ich muss halt so akzeptieren

16 wie das.

Yes, is sometimes annoying when you simply forget something or not...Then that is annoying, but it's not as if I then want to kick myself out of anger. That is not the case. But I need to accept this how it is.

17 LB: Neue Frage: in der Literatur wird häufig gesprochen von erfolgreichen Altern. Was

18 würdest darunter verstehen.

...:in the literature the concept of successful ageing is frequently discussed. What would you understand by this?

$19[\ldots]$ 
1 Lars Bühring: Ich denke das ist wenn man der jeweiligen konkreten Situation angemessen

2 sich auch fühlt und vor allen Dingen eben nicht dem gestern eine so überragende Bedeutung

3 beigemisst. Dass das gestern praktisch halb wie so ein Halbmond halb das jetzt überdeckt

4 oder sogar mehr.

I think that refers to feeling adequately in each respective situation and particularly to not assign such a dominant significance to the yesterday. That the yesterday practically covers, like a half-moon half or more of the now.

$5 \quad$ LB: Und auf dich bezogen: würdest du denken, dass du erfolgreich alterst?

And with reference to you: do you think that you are ageing successfully?

6 Lars Bühring: Das glaube ich wohl, ja.

I think so, yes.

$7 \quad$ LB: Weil?

$8 \quad$ Because?

9 Lars Bühring: Ja, weil ich zwar durchaus das gestern durchaus zu schätzen weiß und mich

10 teilweise auch damit befasse und das auch mal wieder hervorholen dass ich aber durchaus

11 schon sehr viele Erlebnisse habe die das jetzt definieren.

Yes, because I do appreciate yesterday and also think about it and also bring it up once and again but that I also have many experiences that define the now.

12 LB: Das heißt du lebst jetzt und nicht in der Vergangenheit?

That means you live now and not in the past?

13 Lars Bühring: Ich lebe auch jetzt und nicht nur in der Vergangenheit.

I live also now and not only in the past.

14 LB: Denkst du das man die Veränderungen von denen wir jetzt eben gesprochen haben

15 mental und physisch dass man die aufhalten kann, dass man das in irgendeiner Form

16 kontrollieren kann?

Do you believe that the changes we have just talked about, mental and physical, that you can stop them, that you can control them in some way?

17 Lars Bühring: Ja.

Yes. 
And how?

2 Lars Bühring: Ja, indem man möglichst, möglichst eben aktiv lebt.

By living as actively as possible?

$3 \quad$ LB: Was konkret heißt wenn du aktiv sagst, was genau heißt das?

What concretely means when you say actively, what exactly does that mean?

4 Lars Bühring: Ja, indem man zum Beispiel geistig bestimmt bestimmten Ansprüchen, 5 bestimmte Ansprüche oder nein indem man Aktivitäten die bestimmte geistige Ansprüche 6 stellen, durchführt, macht und indem man überhaupt Aktivitäten durchführt, eigene, und 7 nicht nur auf irgendwas reagiert und vor allen Dingen, zum Beispiel, eben seinen, seinen 8 Erlebnishorizont nicht im Fernsehen sieht.

Yes, by living as actively as possible...Yes, for example, by having certain expectations or, no, by executing certain activities which demand mental effort and by executing activities at all, own, and not by just reacting to something and, first and foremost, by not, for example, seeing one's realm of experiences in watching TV.

9 LB: Hat das Altern also der Prozess des Alterns in irgendeiner Form einen Einfluss gehabt

10 auf dein Verständnis von deiner Männlichkeit?

Has ageing, well the process of ageing had in some way had an influence on your understanding of masculinity?

11 Lars Bühring: Das ist eine ganz schwierige Frage. Ich glaube das ich insbesondere was, 12 was diesen Aspekts, nicht Aktivität, sondern was den Aspekt ich sage mal leiten oder 13 bestimmen angeht, dass das dazu geführt hat dass ich das in seiner Bedeutung vielleicht dann 14 doch etwas zurücknehme. Das ist eigentlich für mich der wesentliche Punkt was auch mit 15 etwas anderem zusammenhängt, dass ich nicht meine dass das was ich mir so vorstelle und 16 was so passieren sollte, dass das also sozusagen in Stein gemeißelt ist, sondern das ist also 17 durchaus auch andere Sichtweisen gibt und auch andere Menschen nach einer anderen 18 Sichtweise durchaus auch glücklich und zufrieden sein können.

That is a really difficult question. I think that I especially with regards to this aspect of, not activity, but with regard to the aspect of leading or dictating, that that has led to me taking back its significance a little. That is the crucial point which is linked to something else that I don't believe that what I think and what I believe should happen is written in stone but that there are different views and that other people who see things differently can also be happy and content. 
$1 \quad$ LB: Das heißt du bist toleranter und flexibler als du es vielleicht früher warst?

That means you are more tolerant and flexible than you were previously?

2 Lars Bühring: Ich weiß nicht ob ich toleranter bin aber vielleicht bei so gewissen Dingen

3 reagiere ich nicht so schnell und nicht so plötzlich. Vielleicht im Endeffekt genauso wenig

4 tolerant wie sonst aber bei manchen Dingen kann ich mir sehr viel mehr anhören als das

5 früher der Fall.

I don't know if I am more tolerant, but I don't react as quickly and suddenly to some things. Possibly as little tolerant as I used to be but with regards to certain things I can listen to much more than that was the case in previous times.

$6 \quad$ LB: Und das ist auch etwas, was du als positiv empfindest im Bezug auf Alter?

And that is something which you find positive about ageing?

7 Lars Bühring: Das empfinde ich als angenehm.

That is pleasant.

8 LB: Gibt es andere Dinge die du, wo sagen würdest das ist das Schöne am älter werden.

Are there other things which make you say that is nice about getting older?

9 Lars Bühring: Ja, ich meine, das ist eigentlich, das ist eigentlich so auf den Punkt gebracht,

10 dass man zwar auf der einen Seite sich durchaus an vielen auch kleinen Dingen sehr freuen

11 kann, ohne dass man sich über viele kleine Dinge so ärgert wie das früher der Fall war.

Yes, well, to put it in a nutshell, that on the one hand, you can enjoy many also small things without feeling annoyed by many small things as it was the case earlier in your life.

12 LB: Gibt es Dinge die dich stark beunruhigen am älter werden?

Are there things which majorly worry you about getting older?

13 Lars Bühring: Ja.

Yes.

$14 \quad$ LB: Zum Beispiel?

For example?

15 Lars Bühring: Angst vor Schmerzen - ist eigentlich das bewegt, dass, dass Durchgängige.

16 Oder sagen wir mal vor dem, vor diesem Prozess der vor dem Sterben stattfindet. 
Fear of pain - that is really what moves, the continuous. Or, let's say, the process which happens before dying.

$1 \quad$ LB: Also bedeutet auch das du möglicherweise nicht mehr Kontrolle hast?

Does that also perhaps mean that you don't have control anymore?

2 Lars Bühring: Ja alles, alles das, nicht. Das du dann in eine Situation kommst die für dich,

3 ob nun physisch oder psychisch oder beides, schon sehr unangenehm ist und du aber selber

4 nicht mehr in der Lage bist in irgendeiner Weise darauf einzuwirken oder aber auch nur das

5 zum Ausdruck zu bringen.

Yes, all, all that, no. That you are then in a situation that for you, if physically or mentally or both, really is very unpleasant and in which you are not able to influence or even just to express this.

6 LB: Was, ich weiß das ist eine furchtbare Frage aber was wäre denn letztlich schlimmer für

7 dich, das du sozusagen keine Kontrolle mehr hast über Körper oder über Geist? Also

8 zusammen ist klar, aber wenn du quasi die Wahl hättest...

...what would be worse for you, that you didn't have control over your body or over your mind?...

9 Lars Bühring: Wenn man sich das vorstellt. Wenn du keine Kontrolle mehr über den Körper

10 hast dann nimmst du das ja geistig durchaus wahr. Insofern hat das wieder so eine

11 Konsequenz auf die Befindlichkeit des Geistes und deshalb, also das finde ich schon, also

12 wenn man körperlich verfällt das finde ich schon sehr schlimm muss ich sagen weil das also

13 sozusagen automatisch eben auch was ganz Schlimmes für den Geist ist, ich das nicht

14 ertragen kann.

When you imagine that. When you have no control over your body, then you are still aware of this. As such it will have an effect on the mental state, and that is why, I believe, well, if you decline physically, I find that really very bad, I have to say since that automatically also is something really bad for the mind, I cannot bear that.

$15 \quad[\ldots]$

16 LB: Setzt du dich, also jetzt haben wir ja darüber gesprochen was vor dem Sterben passiert,

17 setzt du dich mit dem Sterben also dem Tod auseinander?

...do you think about dying and death?

18 Lars Bühring: Nein.

No. 
LB: Weil?

Because?

2 Lars Bühring: Weil ich denke, ich denke nicht nach dem Tod.

Since I think, I think not after death.

$3 \quad$ LB: Das heißt, du bist, bist du gläubig?

...Do you believe in God?

4 Lars Bühring: Nein.

No.

$5 \quad$ LB: Gar nicht?

Not at all?

6 Lars Bühring: Finde ich alles völlige Hilfswerkzeuge, die, die menschliche Angst in

7 irgendeiner Weise kanalisieren und verringern sollen aber eigentlich nur ein

8 Machtinstrument von irgendwelchen anderen Leuten darstellen.

... auxiliary tool which is aimed at channelling and decreasing the human fear but really it is an instrument of power for some people.

$9 \quad$ LB: Kirche?

Church?

10 Lars Bühring: Ja, Kirche wie immer du das nennst, Schamanen, Dings...

Yes, church, however you call that, shamans, stuff...

11 LB: Aber du, also ich möchte dir da nicht, nicht zu nahe treten, aber du bist aber schon der

12 Ansicht quasi das ist es. Ein Leben und dann ist Leere.

13 Lars Bühring: Nein sagen wir mal so meine, meine Erkenntnistiefe oder - Weite, die geht 14 nicht über den Tod hinaus. Ich kann nicht sagen da gibt es nichts anderes oder es ist nichts 15 oder sowas. Es ist mir nicht gegeben da für mich einen für mich befriedigenden Kosmos zu

16 erschließen, wo ich sagen kann ja da finde ich mich wieder. Das ist ...es ist einfach eine

17 geschlossene Tür. 
...my depth or breadth of knowledge does not spread beyond death. I cannot say there is nothing else or there is nothing or something like that. I cannot access a for me satisfying cosmos which I can say I can find myself in. That is...it is a closed door.

$1 \quad[\ldots]$

2 LB: Hast du, um mal wieder auf was Positiveres zu kommen, hast du Ziele, Wünsche für

3 die Zukunft, kurzfristig, mittelfristig, langfristig?

...Do you have aims, wishes for the future...?

$4 \quad[\ldots]$

5 Lars Bühring: Es ist also, aber sonst so für mich...ich muss jetzt unbedingt noch mit 85

6 Skifahren oder ich weiß nicht irgendwas, das muss ich alles nicht. Das weiß ich nicht.

7 Irgendwann höre ich das auf.

...I don't have to necessarily go skiing until I am 85 or something. I don't need all that. At some point I will stop that.

$8 \quad[\ldots]$

9 LB: Hast du den Eindruck das jüngere Menschen sich dir gegenüber aufgrund deines Alters

10 in einer anderen Weise verhalten als gegenüber Jüngeren oder Gleichaltrigen?

Do you have the feeling that younger people behave differently towards you because of your age than they do with people their age or younger?

11 Lars Bühring: Ich glaube schon, ein bisschen. Ich glaube schon.

I think so, a little bit. I think so.

$12 \quad$ LB: Und findest du das angemessen?

And do you find that appropriate?

13 Lars Bühring: Ja, die Art und Weise, ich muss wieder darauf zurückkommen auf diese

14 beiden Tenniskollegen, wie die sich mir gegenüber verhalten ist auf der einen Seite völlig

15 auf gleicher Ebene aber in, bei bestimmten Sachen mit einem gewissen aber durch sehr

16 unterschwelligen Respekt. Und das ist also eine angenehme Geschichte.

Yes, the way, I have to come back to these two tennis mates of mine, how they behave towards me is on the one hand totally on the same level but in, with some other things characterised by a certain very subliminal respect. That is a very pleasant thing. 
1 LB: Denkst du dass du in bestimmten für Bereiche des Lebens im Vergleich zu jüngeren

2 Menschen im Vorteil bist und wenn ja wo?

Do you think that in particular areas you are at an advantage compared to younger people?

3 Lars Bühring: Das wäre schlecht wenn das nicht der Fall ist.

That would be bad if that wasn't the case.

$4 \quad$ LB: In welchen Bereichen ist das denn so, denkst du?

In which areas is that the case, do you think?

5 Lars Bühring: Erstmal in den Bereichen wo es um Beziehungen zwischen Menschen geht.

6 Die Einschätzung wie man mit diesen, diesen menschlichen Beziehungen wie man damit

7 umgeht. Damit, da habe ich mit Sicherheit ein bisschen mehr und das alles heißt Erfahrung.

8 Überhaupt wie man, ich sage mal, wie man mit Problemen halt umgeht, wie man wie man

9 Probleme angeht, wie man, wie man Sachen, an welcher Seite man eine problematische

10 Sache vielleicht anfängt und vielleicht auf der anderen Seite eben in einer anderen Weise 11 nicht.

First of all in areas which have to do with relationships between people. The evaluation of how to handle these, these human relationships. With this I certainly have a bit more and that all is called experience. In general how to deal with, let's say, problems, how to go about problems, how to perhaps start a problematic task and on the other hand in another way perhaps not.

$12[\ldots]$

13 Lars Bühring: Gibt es Bereiche wo du denkst das jüngere Menschen dir gegenüber im

14 Vorteil sind?

Do you think that there are areas in which younger people are at an advantage compared to you?

15 Lars Bühring: Tja, so wie ich die augenblickliche Umgebung sehe bedingt, bedingt. Das

16 finde ich eigentlich... Also ich meine wenn sagen könnte die haben das ganze Leben noch

17 vor sich und Dings und so aber was das Leben bringt weiss nicht das ob das so ein großer

18 Vorteil ist. Also, in irgendeiner Weise, also ich kann dazu insofern schlecht, schlecht

19 Stellung nehmen als ich sowas ach das möchte ich auch nochmal oder so. Das kann ich so

20 nicht nachvollziehen. Das ist für mich kein Kriterium. Also ich möchte jetzt keine, keine

21 Familie großziehen oder irgendeinen einen beruflichen Weg durchgehen das möchte ich

22 alles nicht. 
Well, the way I see current society to a limited degree. That I really believe...Well, I think, if you could say they still have their life ahead of them and such things but what life offers, I don't know if that is such a big advantage. Well, in some way, I can't really, I really can't take a stand on this since ...I cannot relate to that. That is not the criteria for me. Well, I don't want to bring up a family or have a career now. I don't want all that.

$1 \quad[\ldots]$

2 LB: Glaubst du das es für Männer oder für Frauen schwieriger ist zu altern?

Do you think it is more difficult for men or women to age?

3 Lars Bühring: Für Frauen ist das viel schwieriger.

It is much more difficult for women.

$4 \quad$ LB: Weil?

Because?

5 Lars Bühring: Ja, weil ich denke die sind zu einem, in einem anderen Alter, wenigstens in 6 meiner Sicht und denke ich wesentlich mehr mit ihrer Umwelt in einer bestimmten Weise

7 verknüpft also in die Umwelt eingebunden, kann auch sagen unselbständiger und damit also 8 auf die.... ja auf das Echo ihrer Umwelt wesentlich mehr angewiesen als Männer der Fall

9 sind. Und da bei Frauen eben das Äußere auch eine große Rolle spielt ist das Echo wird

10 natürlich immer etwas dünner wenn man etwas älter wird. Das heißt Sie haben also mit...

11 Ich denke mal das ist, die werden etwas größere Schwierigkeiten haben ihr Selbstverständnis

12 beizubehalten.

It is much more difficult for women...Yes, because I think that on the one hand, in a different age, they, at least in my view and I think are much more linked to their social environment in a specific way, well, integrated in their social environment, you could also say more dependent and therefore more dependent on the echo ${ }^{31}$ of their social surroundings than it is the case with men. And also since the appearance plays a crucial role and the echo naturally becomes less when one becomes a little older. That means they have with ... I think that is; they will have more difficulty in keeping their self-concept.

$13[\ldots]$

14 LB: Wie würdest du die generelle Einstellung unserer Gesellschaft zum Alter beschreiben? How would you describe the general attitude of our society towards older age?

31 'Echo' in the German language can be used to describe social recognition 
1 Lars Bühring: Die generelle Einstellung hierzulande zum Alter ist meines Erachtens nicht

2 so sehr geprägt von Würdigung dessen was irgendjemand in seinem Leben gemacht hat,

3 auch nicht von einer gewissen Höflichkeit gegenüber dem Alter sondern das ist also ich sage

4 mal weitgehend auch geprägt durch eine wirtschaftliche Denkweise das halt Alter eigentlich

5 halt wirtschaftlich wenig, wenig zu bieten haben oder wenig Belang haben, wenig

6 Bedeutung haben. Was sich vielleicht jetzt so ein bisschen verschiebt wenn man daran denkt

7 dass potenzielle oder potente Konsumenten natürlich gerade alte Leute sind, nicht? Aber

8 ansonsten werden...wird das ja also mehr so das die nichts, nichts mehr zum Sozialprodukt

9 beitragen und dergleichen, also das die mehr oder weniger doch so auf einem dünner

10 werdenden Duldungsakt, ast sitzen.

The general attitude towards older age in this country is, to my mind, not characterised by the appreciation of what somebody did in his life, also not by a certain amount of cordiality towards older age but predominantly characterised by an economic way of thinking that, well, older age has to offer little economically or has little importance, little significance. This might now be changing a little when you consider that especially older people are potential or potent consumers, aren't they? But besides that, ... it is more so that they don't contribute anything to the Gross National Product and something like that, so that they more or less live on a thinning tolerance level.

11 LB: Weil sie nichts mehr beitragen quasi zur Gesellschaft.

Since they don't contribute to society.

12 Lars Bühring: Und damit sind wir bei wirtschaftlichen Gesichtspunkten. Und sowas wie 13 das bei Chinesen ich weiss nicht bei Indern aber bei Chinesen ganz sicher der Fall ist, die 14 also, oder auch in afrikanischen Gesellschaften die also dem Alter einen hohen Stellenwert 15 beimessen und das Ehrfurcht betrachten und die Ratschläge von alten was zu deuten haben... 16 Ich glaube das spielt bei uns in der Gesellschaft auch deshalb keine Rolle weil ja im 17 Augenblick angesagt ist sich immer bloß möglichst der neuesten Entwicklung anzuhängen.

18 Was also heißt das also das was alt ist also wirklich wie der Name sagt alt.

And that brings us to economic aspects. And something as with the Chinese...who or also in African societies that ascribe a high value to older age and view it with respect and in which the advice of older people has value ...I think that this doesn't play a role in our society also since at the moment it is fashionable to under all circumstances follow the latest trend. That means what is old is, how the name prescribes, old. $[\ldots]$

20 LB: Ok und, wo du auch gerade gesagt hast, das ist so eine Tendenz, wo und wie werden denn diese Einstellung vermittelt, denkst du? 
...how and where are these understandings transmitted, what do you think?

1 Lars Bühring: Die werden zum Beispiel vermittelt in der Werbung, die werden vermittelt 2 auch in der Gesetzgebung. Also in der Sozialgesetzgebung oder so was da werden die 3 werden die auch vermittelt. Die werden auch vermittelt durch Institutionen wie die Kirche 4 oder sowas.. Das also... Es wird also an allen verschiedenen Stellen nie vermittelt dass der

5 älteren Teil der Bevölkerung eben ein Teil der Bevölkerung ist sondern, wobei alle umwertig 6 auf der gleichen Ebene sind. Sondern es wird eben immer davon ausgegangen dass 7 sozusagen das Alter halt abrutscht in irgendeiner Weise und deshalb also irgendwie einer 8 besondere Betrachtung nur entspricht oder eine besondere Betrachtung verdienen. Ja und 9 eigentlich die Jungen und Aktiven die sind halt der Maßstab. Und wenn der Maßstab halt so 10 ist dann ist das klar das Alte dann in einer bestimmten Weise dann abgewertet werden.

They are, for example, transmitted in advertising, they are also transmitted in the law. Well, in the social legislation or something like that, there they are also transmitted. They are also transmitted by institutions such as the church or so...That well...At all different levels, it is never transmitted that the older part of society is part of a society in which are all equally on the same level. But it is always assumed that older age means a decline in some way and that is why it only conforms to or deserves a special examination. Yes and really the young and active are really the measure. And when that is the measure, then it is clear that the old are in some way devalued.

11 LB: Und Werbung hast du auch erwähnt, hast du da irgendwas Spezielles im Kopf, 12 irgendeine spezielle Werbung so als Beispiel oder so eine Tendenz die du wahrnimmst wenn 13 es um Werbung geht?

And you mentioned advertising, do you have something specific in mind, a specific ad as an example or a tendency that you have noticed with regards to advertising?

14 Lars Bühring: Ich finde dieses Bild, was in der Werbung vermittelt wird das entweder sind 15 das immer kranke Menschen denen durch irgendwas geholfen wird oder aber Leute denen 16 auch immer irgendwie geholfen wird damit sie glücklicher werden. Das heißt sie befinden 17 sich immer in einem Zustand der irgendwie bemitleidenswert ist oder noch nicht mal 18 bemitleidenswert, auf alle Fälle Anlass gibt dass man ihn verbessern könnte also dass es 19 jemanden gibt der ohne Beschwerden alt und glücklich ist das gibt es in der Werbung nicht.

I find this image which is transmitted in advertising that there are either ill people who are helped or people that are also helped by something so that they are happier. That means they are always in a condition that is somewhat pitiable or not even pitiable but definitely give a reason for improving it so that there is somebody who is old and happy without ailments that doesn't exist in advertising. 
1 LB: Wobei man das dazu sagen muss grundsätzlich bei der Werbung so ist, das ist ja der

2 Job der Werbung...

...that is the job of advertising...

3 Lars Bühring: Ja natürlich aber das ist eben bei älteren Menschen besonders, besonders 4 stark.

Yes, of course, but with older people, it is especially, especially strong.

5 LB: Allerletzte Frage: du hast ja schon gesagt dass also in Asien ein anderes Verständnis 6 von Alter herrscht, denkst du dass es auch spezifisch in Deutschland also wirklich auch im

7 Hinblick auf deutsche Kultur, sich das Altersverständnis unterscheidet von, sagen wir mal

$8 \quad$ Italien oder also Europa?

...do you think that the understanding of ageing in specifically Germany is different to, let's say, to Italy or, well, Europe?

$9 \quad$ Lars Bühring: Ja, glaube ich ganz dicke.

Yes, I definitely think so?

$10 \quad$ LB: Ja, und was meinst du ...?

Yes, and what do you think...?

11 Lars Bühring: Weil hier, weil der, außer vielleicht in den USA aber in keinem der großen

12 zivilisierten wirtschaftlich potenten Ländern zu denen Deutschland an vorderster Stelle zählt

13 gibt es ein derartiges und zwar durchgängiges Verständnis von Leistung. Und wenn man

14 sich die Italiener anguckt oder Franzosen oder Spanier oder so.. Ich meine auch Engländer

15 kann man alles vergessen, die da vielleicht in etwa hinreichen sind Holländer oder Dänen

16 oder sowas aber sonst in dem Augenblick in dem dieses, dieses Leistungsprinzip eine

17 besondere Bedeutung, eine ganz überragende Bedeutung hat ist klar, dass derjenige der

18 nicht mehr leistet auch in der sozialen oder in der Anschauung der Bedeutung für die

19 Gesellschaft natürlich nicht an vorderster Stelle rangiert.

Since here, since it, except perhaps in the US but in no of the big civilised economically potent countries within which Germany takes a leading position, there is such a and so consistent perception of efficiency... in the moment in which this achievement principle has a special significance, an outstanding significance it is clear that he who cannot achieve anymore also in the social or in the perception of the meaningfulness for society obviously doesn't take a leading position. 


\section{K Concept-Map Thematic Units Masculinity \& Femininity}

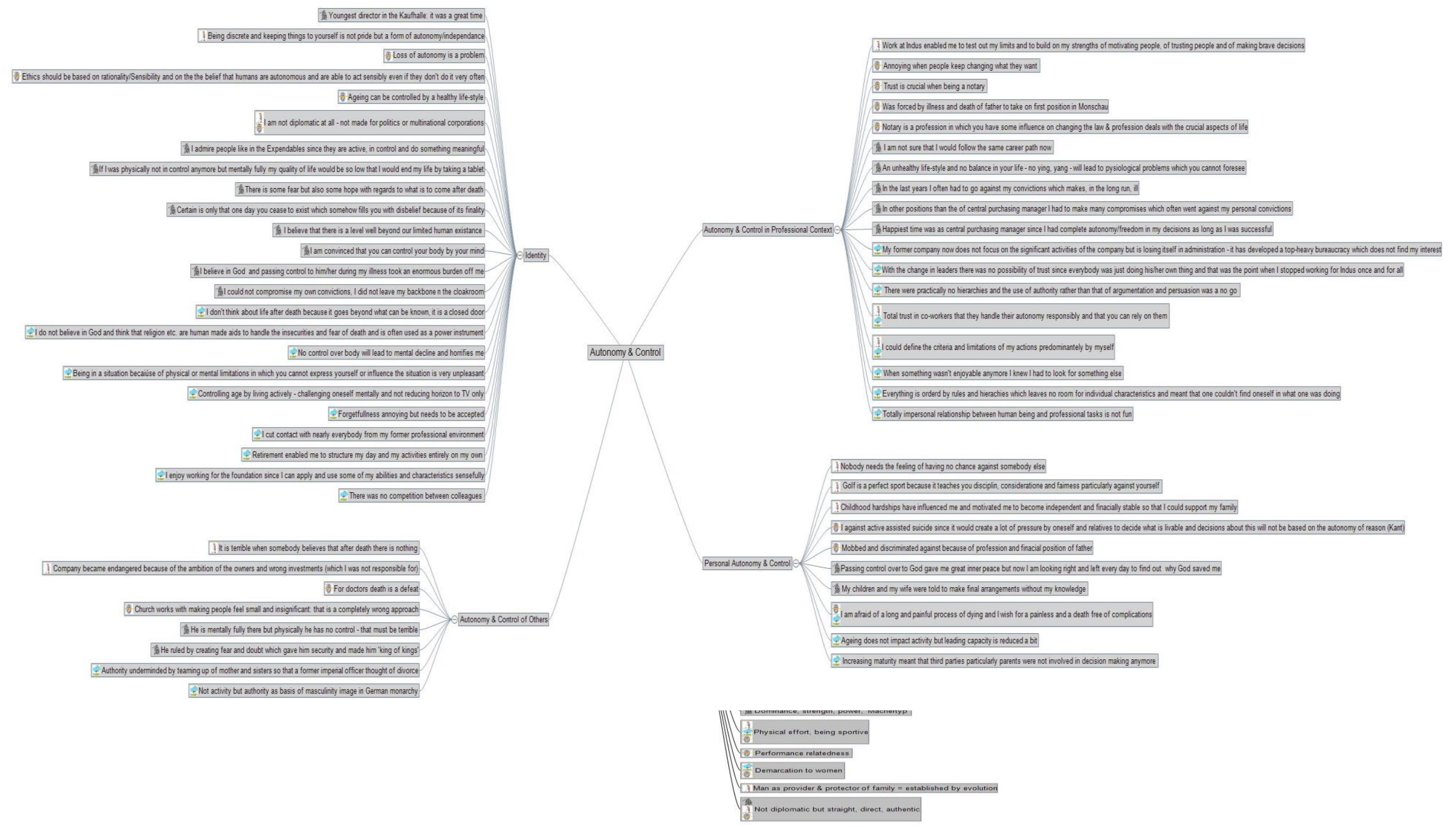

\section{Concept-Map Thematic Units Control \& Autonomy}




\section{Concept-Map Thematic Units Ageing as Decline}

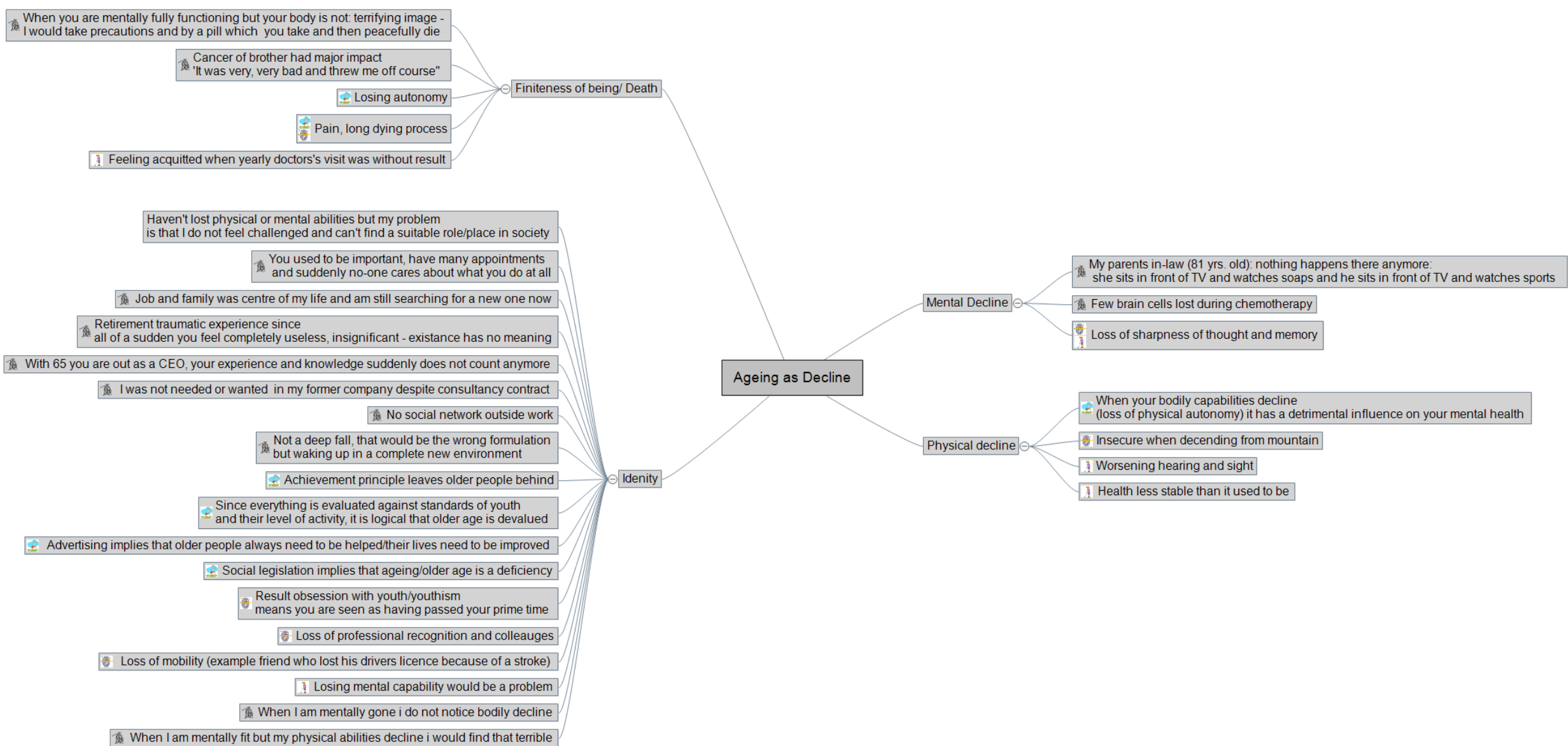




\section{N Concept-Map Thematic Units Successful Ageing}

Toring to regular check ups helps to control the dangers of physical deccine and illiness

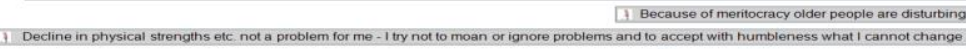
Having more time a asso to meet trends, tamil 1 Enjoy verery day and its itite pleasures

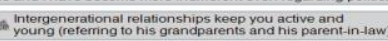

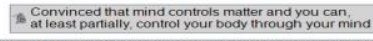

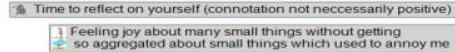
You can control age when you stay active
to contront your F. Level of action and reaccion more leveleedbatanced than 4 used to

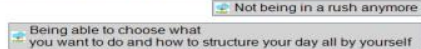
Ij Focus on signiticant thing

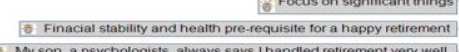
10 My son a psycholoogsts, alvays says inandeded retrement ver well

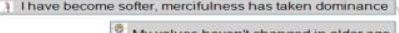
5 My values havent changed in older age

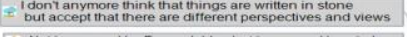

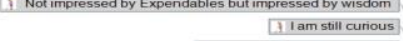

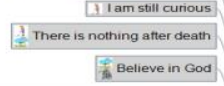

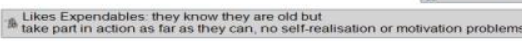

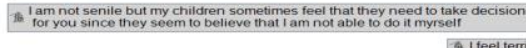

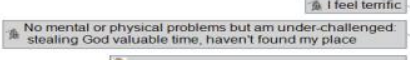

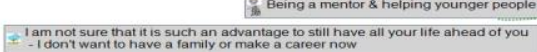

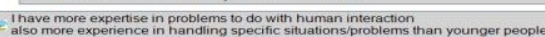

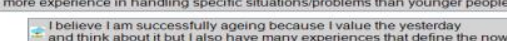

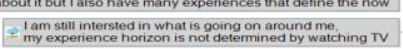

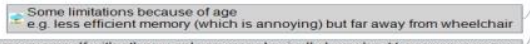
F. When I compare myselt with other people my age physicalty $y$ a a mabout ten years younger

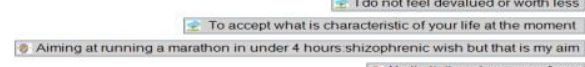

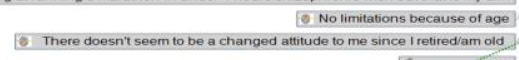
is idont ree old

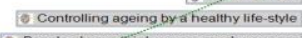
Retrementorider age

Successful Ageing

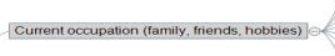

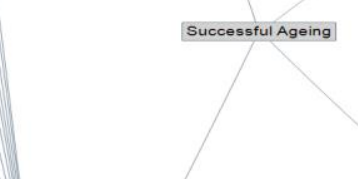

Current occupation (rortessionall]
Being asked tor helpatavice by younger peopie - Wite is intelefectual patrerer many discusussons Travelling F Prilosopopy

Doing sports Staying physically it

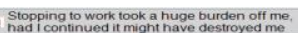

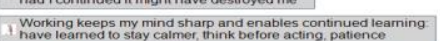

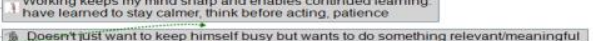

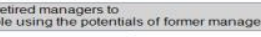

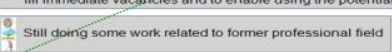

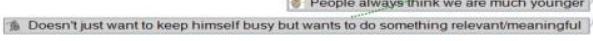




\section{O Concept-Map Thematic Units Society, Culture \& Media}

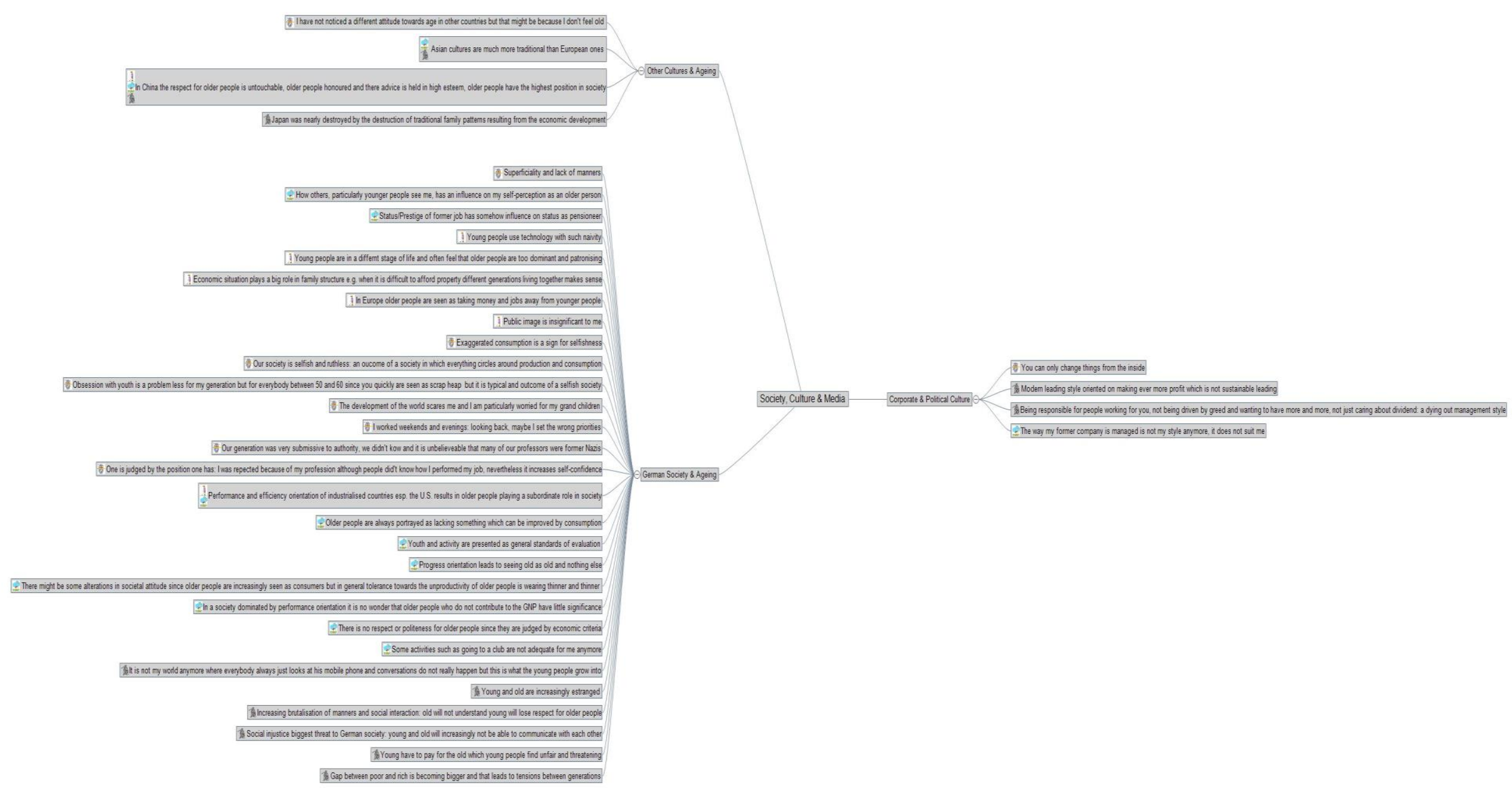




\section{P Concept-Map Thematic Units Generational Conflict}

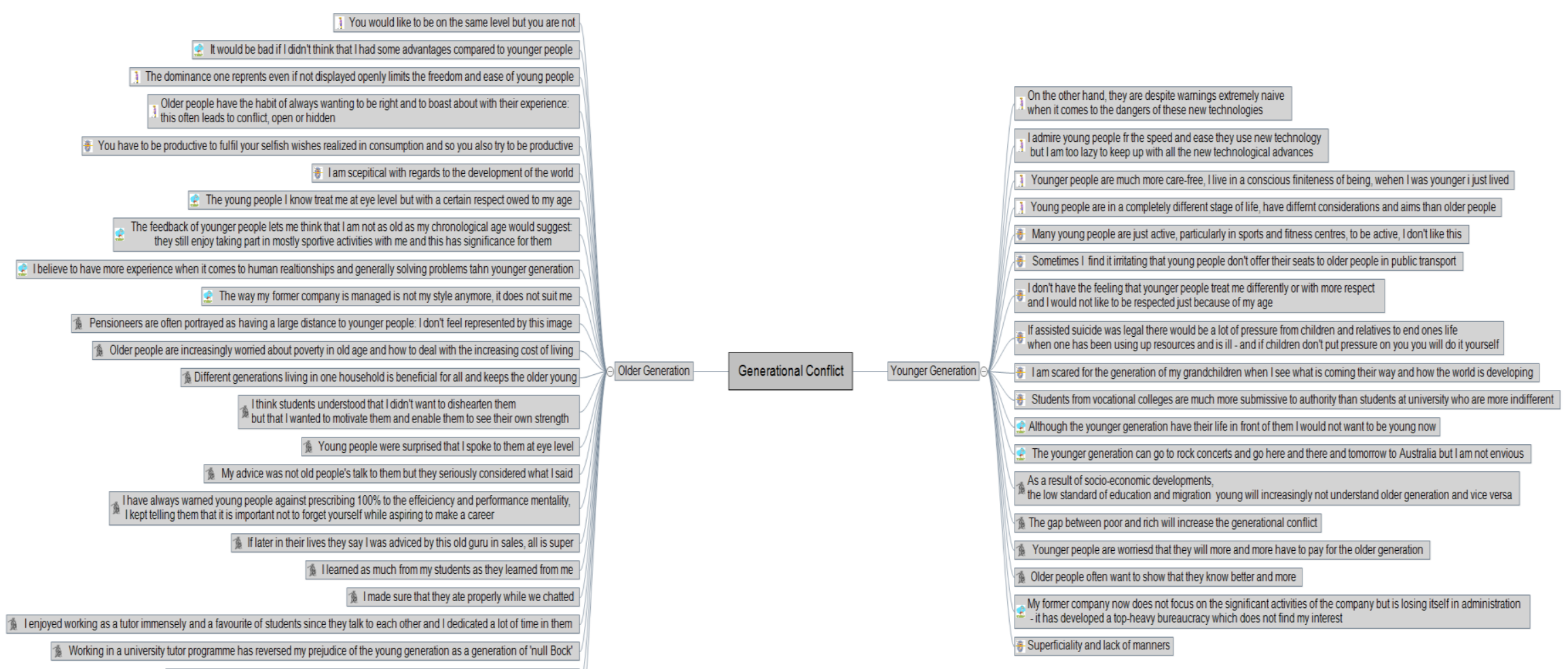

